Mirosława Marody/Anna Giza-Poleszczuk

\title{
Transformations \\ of Social Bonds
}

The Outline of the Theory

of Social Change

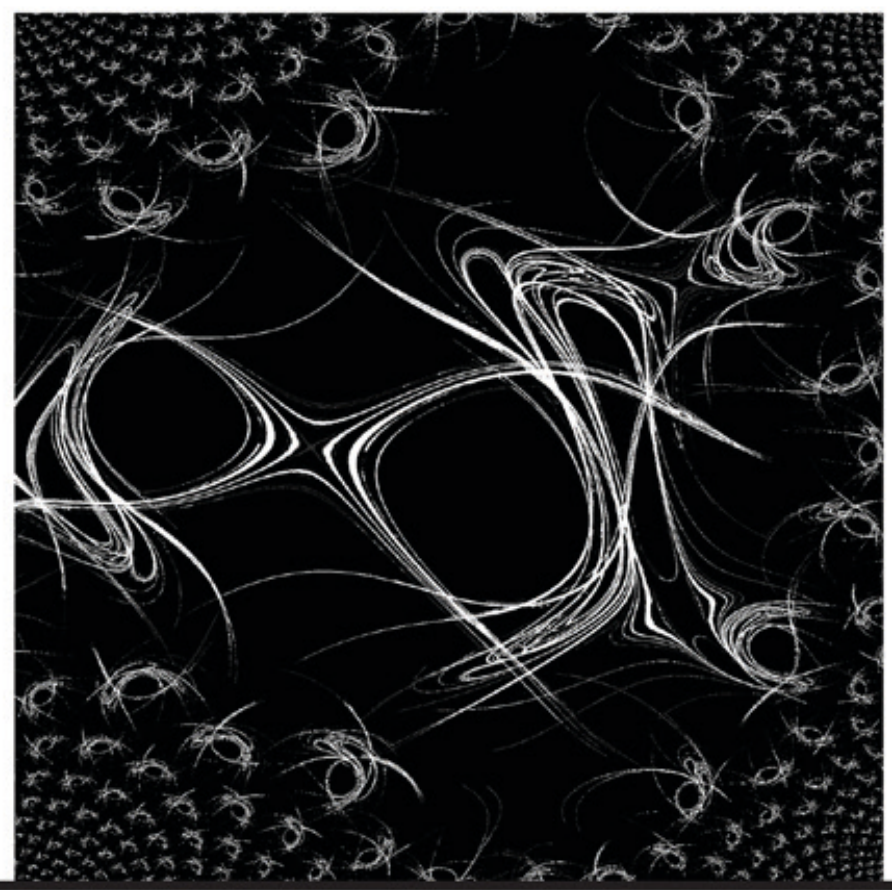




\section{Mirosława Marody/Anna Giza-Poleszczuk}

\section{Transformations of Social Bonds}

We live in times of accelerated changes - social, economic, and political - which are currently transforming most societies and areas of human life. Every day brings not only new shifts in political orders of various countries but also clearly observable metamorphoses of labour patterns, family forms, and modes through which we participate in political life, communicate, and which are related to each other. The pace of these changes and their complex mechanisms make it difficult to discern what directions they are actually taking. This book is about transformations of social bonds, the most fundamental sociological concept. It examines how these bonds are formed, how they are dissolved, and how they are forged anew. The book offers a preliminary reflection on the course and consequences of the ongoing transformations of the social order. At the same time, it invites to reconsider the foundations of sociological thinking.

\section{The Authors}

Mirosława Marody is a Professor of sociology and social psychology in the Institute of Sociology at the University of Warsaw and the head of the Centre for Political Studies in the Institute for Social Studies. Her recent works focus on transformation processes in contemporary society. She has published many books on systemic transformation in Poland.

Anna Giza-Poleszczuk is a sociologist, Professor at the University of Warsaw. She has accompanied Polish transformation both as a scientist and practitioner. As a researcher, she was involved in many local and international projects, resulting in a few important books and publications. As a practitioner, she worked for an international company managing consumers' insight department and advising to the Board, designing and evaluating many NGOs programs. Since 2012, she has served as a Vice Rector for the Development and Financial Policy at the University of Warsaw. 
Transformations of Social Bonds 

Mirosława Marody, Anna Giza-Poleszczuk

\section{Transformations of Social Bonds}

The Outline of the Theory of Social Change

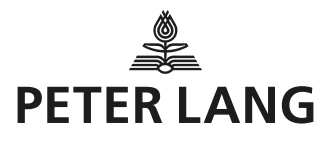


Bibliographic Information published by the Deutsche Nationalbibliothek The Deutsche Nationalbibliothek lists this publication in the Deutsche Nationalbibliografie; detailed bibliographic data is available online at http://dnb.d-nb.de.

\section{Library of Congress Cataloging-in-Publication Data}

A CIP catalog record for this book has been applied for at the Library of Congress.

The Publication is funded by Ministry of Science and Higher Education of the Republic of Poland as a part of the National Programme for the Development of the Humanities. This publication reflects the views only of the authors, and the Ministry cannot be held responsible for any use which may be made of the information contained therein.

Years of funding: 2016-2018.

U national programme FOR THE DEVELOPMENT OF HUMANITIES

Translated by Grzegorz Czemiel

Cover image: Courtesy of Benjamin Ben Chaim Cover Design: (C) Olaf Gloeckler, Atelier Platen, Friedberg
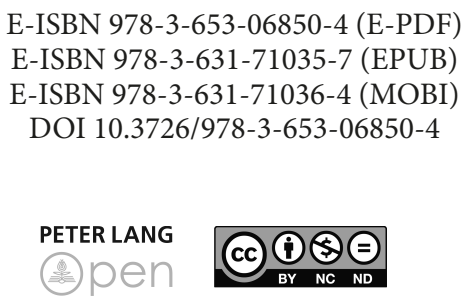

Open Access: This work is licensed under a Creative Commons Attribution Non Commercial No Derivatives 4.0 unported license. To view a copy of this license, visit https://creativecommons.org/licenses/by-nc-nd/4.0/

(C) Mirosława Marody/Anna Giza-Poleszczuk, 2018

Peter Lang - Berlin · Bern · Bruxelles · New York · Oxford $\cdot$ Warszawa $\cdot$ Wien

This publication has been peer reviewed. www.peterlang.com 


\section{PREFACE TO THE ENGLISH EDITION}

We live in times of accelerated changes - social, economic, and political - which are currently transforming most societies and areas of human life. Every day brings not only new shifts in political orders of various countries but also clearly observable metamorphoses of labor patterns, family forms, and modes through which we participate in political life, communicate, and are related to each other. All these things constituted a seemingly stable foundation of social life in the past, but this is no longer the case. The pace of these changes and their complex mechanisms make it difficult to discern what directions they are actually taking.

Such a cognitive confusion has also engulfed sociologists. Some scholars note the crisis of society or even its demise, while others are equally assertive in announcing the birth of a new form of social life: the postmodern society, and yet others maintain that we are witnessing the crisis of sociology itself. Fragmented into a myriad of specialized research programs, the discipline seems unable to develop any generalized image of either contemporary reality or social change as such.

At the onset of the second half of the twentieth century, sociology seemed convinced that it has at its disposal the tools necessary to understand, or perhaps even manage, the social and economic reality. Today, however, instead of peacefully reaching the end of history, at which point the secular model of the world would materialize in the form of a democratic, knowledge-based society of individuals, we witness unexpected twists and turns, which involve the disappearance of social forms that seemed to be firmly grounded, accompanied by the re-emergence of forms long deemed obsolete. The prevailing sociological model of the society either ignores these phenomena or seeks to explain them in terms of ad hoc conceptions, anchored in common-sense knowledge.

Thus, when we became interested in processes of social change, we decided to return to the sources of sociology and its most fundamental concepts or assumptions - ones that provide the foundation on which the discipline was built. Among them, a crucial role was played by the conviction that social changes have a discontinuous and nongradual character, that they did not follow any higher logic of development, and that subsequent "incarnations" of social order are not simple continuations of the previous order on a higher level of complexity. The overarching concept that determined classic sociological analyzes was that of social bonds: relational linkages tying various elements together to form a larger whole capable of coordinated action and causing individual actions to be, consciously or unconsciously, subordinated to the realization of common good. 
This book attempts to answer how these bonds are formed, how they are dissolved, and how they are forged anew. It offers a preliminary consideration of what affects us all these days, regardless of any specific conditions, a reflection on the course and consequences of the ongoing transformations of the social order. At the same time, it invites to reconsider the foundations of sociological thinking. Today, sociology needs this kind of debate more than any time before.

Fourteen years ago, when we started our work on this book in Polish, the processes whose consequences we are experiencing so powerfully today, have only begun to come to light. However, already at that point it was quite clear for some sociologists that we are actually dealing with changes leading to the "end of the world as we know it." Today, the ground-breaking works by Ulrich Beck, Anthony Giddens, Zygmunt Bauman, Immanuel Wallerstein or Manuel Castells - which were also for us a source of inspiration - are part of the sociological canon. Unfortunately, their impact on sociological research has been rather minor, since the conceptual apparatus of contemporary sociology still remains unable to grasp those processes of change that affect the very basis of social organization of life.

At the same time, however, the basic factors influencing such a change - ones which we identified fourteen years ago - are now gaining ever greater importance within the processes that mark "the beginning of a world we are still learning to recognize." This bolsters our courage to introduce this book - despite its age - to the English-speaking public.

For the purposes of the English edition, we have slightly altered the composition of the book. The original chapters 1-2, focused on the condition of contemporary sociology, have been shortened into one section whereas the last chapter has been divided into chapters $8-9$.

Warsaw, September 2018

Mirosława Marody Anna Giza-Poleszczuk 


\section{CONTENTS}

INTRODUCTION

CHAPTER ONE. WHAT IS SOCIETY? ............................................. 17

1.1 Classical views on the question of society ............................................. 19

1.2 Society as an object of enquiry in twentieth-century sociology ......... 24

1.3 Society as a theoretical concept ................................................................. 34

\section{CHAPTER TWO. MECHANISMS OF SOCIALIZING}

PROCESSES ………………………………........................................... 41

2.1 Sociality in the perspective of anthropo- and sociogenesis ............................................................................................ 45

2.2 Social foundations of the human mind ................................................ 61

2.3 Two dimensions of socializing processes ................................................ 71

\section{CHAPTER THREE. TRANSFORMATIONS OF SOCIAL}

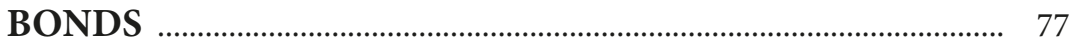

3.1 The nature of social bond .............................................................. 82

3.2 Culture as a "toolkit" ............................................................................ 92

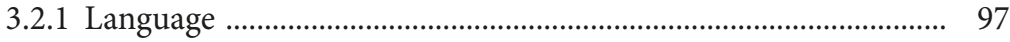

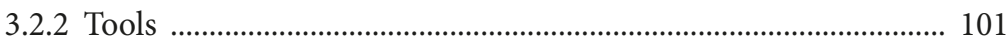

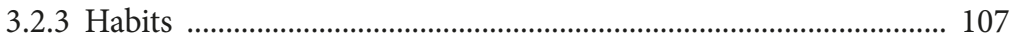

3.2.4 Factors determining the transformations of culture ….................. 112

3.3 Transformations of social bonds ............................................................ 116

CHAPTER FOUR. SOCIETY AS A PROCESS …........................... 127

4.1 Levels of analysis ................................................................................... 131 


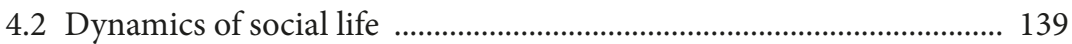

4.3 Analyzing processes of historical changes .............................................. 153

\section{CHAPTER FIVE. TRANSFORMATIONS OF THE}

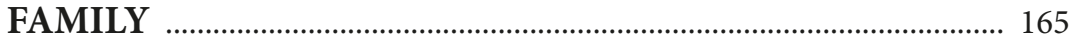

5.1 The universe of the concept of family .................................................... 167

5.2 Processes of reproduction .................................................................... 173

5.2.1 Family constellations: societies without history ............................ 177

5.2.2 Lineage, race, and house - constellations of the "traditional" family ............................................................................................... 180

5.2.3 "Family" as a vehicle for the reproduction of social structure: the dominance of the logic of market and state ........... 187

5.3 Flourishing of industrial society and criticism of bourgeois family ..... 191

5.4 Postmodern family ................................................................................... 196

5.5 Crisis of the family as crisis of society ……........................................... 198

CHAPTER SIX. TRANSFORMATIONS OF LABOR .................. 203

6.1 Labor as an element of being in the world - hunter-gatherer

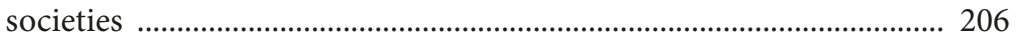

6.2 Labor as a task - traditional societies ................................................ 210

6.3 Denaturalization of labor - modern society …………………………... 225

6.4 Dematerialization of labor in contemporary society ............................. 236

\section{CHAPTER SEVEN. TRANSFORMATIONS OF THE PUBLIC} SPHERE

7.1 Semantic aspects of the public/private opposition ................................. 246

7.2 The concept of the public sphere ............................................................ 254

7.3 From tribal to political community - traditional societies .................. 263

7.4 The public sphere in the modern society ............................................... 280

7.5 Contemporary transformations of the public sphere ............................. 287 


\section{CHAPTER EIGHT. MECHANISMS OF GREAT}

TRANSFORMATIONS …............................................................... 291

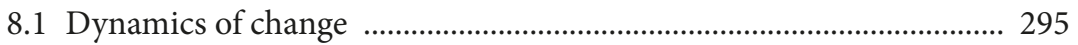

8.2 Transformations of the processes of socializing human actions ........ 306

\section{CHAPTER NINE. TOWARDS THE POSTMODERN}

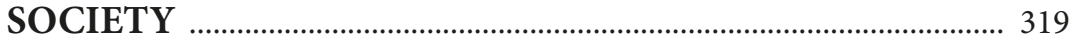

9.1 From a society of local communities to a discreet society ................... 320

9.2 What comes after modernity? ................................................................ 334

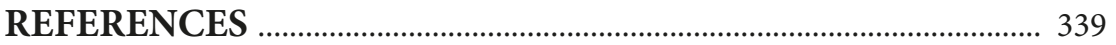

INDEX OF NAMES …......................................................................... 357 



\section{INTRODUCTION}

This book is about social change. This should not surprise anybody since we do, after all, live in a time of accelerated transformations, a topic addressed in some way or another by most sociological studies published these days. This book also focuses on contemporary social processes, raising the question whether we are dealing with a merely "quantitative" intensification of changes, or with some kind of a "qualitative" shift in the forms that organize human behavior - a shift that has been tentatively called the transition from modern to postmodern society.

It is quite astonishing that today's sociology avoids providing an unambiguous answer to this question, or even shies away from positing it. Instead, it limits itself to exploring particular regularities or processes that can be found in social life today. One could even have the impression that sociologists have focused on developing a kind of "sociography" which is able to document the emergence of new phenomena but which remains helpless when it comes to explaining them. These phenomena are usually considered to be either an effect of behavioral trends, which have always been present in modernity and became merely accelerated or intensified by technological progress (such as individualization, rationalization or consumerism), or a consequence of some modifications in the structures of modern society (such as civic society, social trust or democratic systems). In both cases, however, concepts employed in sociological accounts are not framed by a more general theory of social change.

Such an attitude appears all the more astounding when we remember that sociology was founded as an intellectual response to the changes caused by the "transition to modernity." The basic set of concepts developed by the classics of sociology, which determined its status as a separate discipline, was supposed to detect these changes and provide their theoretical explanation, not a mere description. It was supported by the conviction that modern society was born through the emergence and subsequent dissemination of radically novel patterns of social action - "illegitimate" ones, in Weber's phrasing - and not through the maturation or development of trends that have originated in the preceding form of traditional society.

The classics of sociology - like Durkheim, Simmel, Tönnies or Weber - would assume that if there is no identifiable consequential logic, which governs the transformations, the only way to understand the relation between various forms of social organization is to grasp the mechanism or mechanisms that generate and reproduce these forms in the historical process. It was the search for such 
universal mechanisms - operating in diverse contexts and interacting with various factors, ultimately giving rise to different forms of social order - that was meant to constitute the fundamental task of sociology. Each of the abovementioned authors attempted to indicate certain general factors responsible for producing and then sustaining coordination of human actions, assuming the "transition to modernity" to be a historical event - neither the fulfillment of some historical necessity, nor another stage in the development of humanity. Furthermore, each of these authors emphasized that this transition involved a radical shift in the relative importance of factors that integrate human actions, rather than a substitution of one group of factors for another. In other words, the founding force of sociology was the effort to create a general theory of such mechanisms of coordination and integration human actions - after all, these mechanisms constitute the essence of what we call "society" - that have underlain all historical manifestations of sociality.

Thus, when we started our own analysis of social change, it seemed sufficient to revisit classic (albeit nearly forgotten) sociological concepts - such as social bond, sociality, or social action - and employ them so as to order the rapidly expanding mass of descriptions of contemporary society. We thought it would suffice to answer the above question whether we are witnessing the emergence of a new form of social order or merely evolution of modernity's characteristic features. However, it soon turned out that such an approach would be difficult, or even dangerously fallible, for at least several reasons.

First of all, having firmly established themselves in sociological discourse including its didactic, theoretical, and research-oriented subtypes - all those classic concepts became trivialized and clichéd, losing their original meanings. In our view, they are no longer an integral part of the discipline's paradigm but rather an assembly of stereotypes in sociological thinking. Not only do they fail to generate new hypotheses, ideas or experiments but also bring sociology to a standstill, inducing intellectual stagnation. To put it differently, we have developed a habit of reacting to the sound of certain words without taking into consideration their meaning. Indeed, it was already Locke who argued that "society influences the individual." But what did he mean by that? What kinds of mechanisms of influence did he have in mind? How did he understand society? And most importantly, what does it mean today that "society influences the individual"?

Second, as a result of the above, certain classic formulas which seem indispensable to sociology's theoretical discourse have acquired the ability to "lull" their potential recipients. It is sufficient to use the concept of "anomie" to make readers believe that they already know what the text is about and therefore can 
skim through it without paying attention to the actual argumentation. In this situation, attempts to formulate new theses on the basis of such concepts frequently fail because of the tendency to follow the beaten track, fall back on readily available schemas, and pretend not to notice any incongruities. While working on this book, we have often caught ourselves resorting to ready-made formulas which otherwise may be right but which also invariably occlude the nature of the discussed issue. Struggling with the worn-out language of sociology proved to be probably the greatest difficulty in completing this book.

Third, most key ideas and expressions developed within classic sociology have been layered with so many interpretations that it sometimes becomes impossible to establish their original meaning. What is more, a large portion of these explications has a clearly "classificatory" character. Durkheim, for instance, has earned the label of "positivist" and "naturalist," whereas many new readings of classic works focus on proving that, for example, Weber is not only the father of interpretive sociology but also of the rational choice theory. Regardless of the fact that nobody knows to what extent such findings might help us understand what these authors wanted to say, this kind of analysis turns their works into dusty old scrapbooks, full of quotations used to support arguments that often have little in common with the problems they actually explored.

Finally, and fourth, classics of sociology worked at a specific time in history. This also regards a specific state of knowledge in other scientific disciplines, which they used as a point of reference or source of inspiration. Durkheim's works were indebted to the achievements in anthropology of that time, while Weber relied heavily on findings not only in history and economy, but also logic, since - as he explains in a footnote - he would use the concept of value in the sense given to this term by "contemporary logic." Do we really know what sense was that, or are we rather using Weber's concept in accordance with our own, intuitive and blurred understanding of values? Of course, the development of particular sciences during the time that separates us from Durkheim or Weber can mean that most of their claims have ceased to hold ground. However, it can mean something else, namely that the current state of knowledge could help these theoreticians to substantiate and express their theses better. For example, William James would be probably enthusiastic about Joseph LeDoux's The Emotional Brain, while Durkheim and Mead could find it very useful to study the latest findings in neurophysiology, which demonstrate that their theses about the integrating role of ritual and "taking the attitude of the other" have a strictly "physical" basis since the human brain shows signs of imitating other people's actions and emotions while observing them. 
An awareness of these conditions and pitfalls has decidedly shaped this book. Its main goal is to explore social change in terms of dominant forms of "socializing processes," i.e. processes that generate relatively stable patterns of coordination of individual actions. We explore a certain theoretical model of such processes, presenting mechanisms that fundamentally impact their course and pointing to key historical and contextual conditions in which these mechanisms lead to the emergence of different forms of "sociality" that organize social life. Such a delimitation of the scope of inquiry rests upon the assumption that the history of human societies is discontinuous and aimless, while changes that occur within it have a unique and abrupt character. We also assume that historically distinguishable forms of sociality constitute merely models or abstractions from the incessant stream of activities, just like language (langue) is only an abstraction from the stream of speech (parole).

Adopting the socializing processes as a key notion we return to the roots of the sociological thinking developed by the discipline's classics for whom the basic question was how individuals come to coordinate their actions into forms that are, in return, perceived as "social" i.e. external and obligatory. Quoting extensively from their works we wish not so much to support our claims with their authority as to enter a dialogue with those of their findings that are directly connected with our own concerns, and thus recall and refresh them, placing their meaning in new light. At the same time, we wish to strongly emphasize that we neither attempt to develop "the only correct" interpretation of classical sociology, nor wish to assess its findings in terms of truthfulness and falsehood. Our primary goal is to expand on certain theoretical ideas present in them - ones that, to our astonishment, seem more suitable for explaining today's changes than many of the currently developed theories.

When relating the classic findings to the current state of knowledge in other disciplines (primarily those that are crucial for better understanding of basic sociological concepts and models built on top of them) we have tried to implement an important methodological principle that recurs, in different forms, throughout works by almost all authors considered to be the classics of sociology. This principle holds that sociology is, by its very nature, an interdisciplinary science. Thus, in breaking away from history, anthropology, neurophysiology, evolutionary biology, and psychology, it condemns itself to a futile juggling of empty concepts.

The main problem of contemporary sociology is how to grasp the social. Under conditions of intensified change that destroys the hitherto existing social structures, sociology turns from analyzes of social systems, institutions and structural determinants of human actions towards analyzes that focus on individual 
behavior, nature of interactions, and culturally established meanings. As we argue in the first chapter, such a mode of thinking results in an increasing "individualization" of the discipline and de-theorization of its basic concept, which is that of society. A return to some of the original ideas developed by classics of sociology allows to see that both the notions of individual and society were conceptualized by them merely as abstractions from social processes. The meaning and consequences of this statement are examined in three subsequent chapters (2-4), where we also offer our own perspective on socializing mechanisms, i.e. mechanisms that turn individual behavior into streams of coordinated collective actions. This allows us to develop a view of society different than the one currently prevailing in sociology. It ceases to appear as the epiphenomenon of state, politics, economy or culture, and becomes instead an abstract form encapsulated in coordinated actions, thus acquiring the status of a sui generis reality, just like Durkheim wanted.

Three further chapters (5-7) provide an empirical illustration to this model by adapting it to analyzes of socializing processes occurring in three different areas of social reality: family, labor, and the public sphere. Without attempting to summarize those processes, we just wanted to show how the concept of socializing processes can help to problematize anew analyzes made in these areas. This survey of historical changes in family, labor, and the public sphere also provides an empirical basis - one necessarily limited and fragmentary - for more general theses formulated in the last two chapters. They relate changes in three key contextual factors - population, environment, and collective representations, which provide the "environment" in which socializing processes can find expression to changes in basic forms of sociality which remain central to the organization of social life.

Readers should be warned right away that this book does not do justice to contemporary sociology by emphasizing that there are also innovative and inspiring works in this discipline. For our intention was rather to focus on what sociologists - including ourselves - consider to be obvious, i.e. what forms a kind of "collective representation" of our scholarly environment. Producing such "representations" is unavoidable in the era of high specialization marked by a deluge of works offering analyzes of narrow aspects of social life. We argue, however, that once in a while they ought to be reflected upon in order to "deconstruct" the hidden assumptions which inform our discipline.

We do not regard this book to be a finished work but rather an introductory outline of an approach to the study of the social. Therefore, we are aware that more astute readers may find it to contain various shortcomings, and that some of our ideas may undergo vital modifications in the future. The present study 
does not claim to be a "revelation of truth" but rather an invitation to participate in a debate that contemporary sociology seems to need today more than any time in the past.

The importance of such a debate dawned on us as we were working on this book. We began with certain reflections that each of us had collected in the course of individual reading, researching and teaching. Looking back from the perspective of a completed book, reflecting on the concepts and findings it contains, we are fully aware that neither of us could write it on her own. It would not be an exaggeration to say that - with the exception of the fifth chapter, largely based on Anna Giza's work published after this book - this study does not contain even a single idea that, after being introduced by one of us as a topic for consideration, would escape being reworked, enriched and refined in the course of numerous discussions and debates with the other. In this sense, the book constitutes the intellectual property of both authors. The order of names on the title page does not reflect any imbalance in their actual input, but rather documents the fact that one of them took the pain to edit the book and give final form to joint reflections and various passages written at different stages in the book's development.

While working on this book, we enjoyed financial support from the State Committee for Scientific Research $(\mathrm{KBN})$ as part of a research project $(\mathrm{H} 02 \mathrm{E}$ 026 21), which also involved doctoral students Tomasz Płachecki, Maria Rogaczewska and Alina Stanaszek, who prepared partial reports and helped in technical matters related to preparing the manuscript for printing. We wish to express our sincere gratitude to them.

Further acknowledgments are due to some of our colleagues - primarily Prof. Marian Kempny and Prof. Jacek Kochanowicz - who read the early draft and whose remarks made it possible to avoid rash generalizations in many places. We are further obliged to Dr Dominik Batorski, Dr Sławomir Mandes, Prof. Zbigniew Mikołejko, Dr Jan Poleszczuk, and Prof. Marek Zirk-Sadowski, whom we kept bothering with various specialist questions. Naturally, we bear full responsibility for all the passages the reader may deem too controversial. 


\title{
CHAPTER ONE. WHAT IS SOCIETY?
}

\author{
What we are witnessing at the moment is that a certain \\ model has become worn out, while former ways of \\ understanding the world have faded. \\ JEAN-PAUL FITOUSSI \& PIERRE ROSANVALLON, Le Nouvel Age des inégalités \\ If, as is granted to us, this synthesis sui generis, which \\ constitutes every society, gives rise to new phenomena, \\ different from those which occur in consciousnesses in \\ isolation, one is forced to admit that these specific facts \\ reside in the society itself that produces them and not in \\ its parts - namely its members. \\ ÉMILE DURKHEIM, The Rules of Sociological Method
}

Norbert Elias begins one of his works by observing that "[e]veryone knows what is meant when the word 'society' is used, or at least, everyone thinks he knows." "One person," Elias continues, "passes the word on to another like a coin whose value is known and whose content no longer needs to be tested. If one person says 'society' and another hears it, they understand each other without difficulty. But do we really understand each other? [...] What kind of formation is it, this 'society' that we form together, which has not been intended or planned by any of us, or even all of us together? It only exists because a large number of people exist, it only continues to function because many individual people want and do certain things, yet its structure, its great historical transformations, clearly do not depend on the intentions of particular people" $(2001,3)$.

Although Elias posed this question as early as 1939, it remains hard to answer in any binding way. Indeed, the term "society" has entered common usage. Weekly magazines often contain sections under this heading and it appears that using this word has become a natural reflex in public discourse. We have grown accustomed to believe that "society" refers to the entirety of the population in a given country. Moreover, we expect this entity to have certain features, even if we, who belong to it, do not find them within ourselves. Finally, we consider it obvious that "society" goes through certain changes, even though attempts to define the direction and nature of its transformations may spark controversy. We no longer ponder what society is, restricting our efforts to asking what its chief characteristics are. 
Attempts to describe "society" are also usually associated with providing quantitative data, i.e. quoting indicators and distribution of answers in polls conducted invariably on "representative samples" reflecting a given society's composition with regard to basic categories such as sex, age, education, region etc. The most widely applied categories used to segment "society" have statistical, usually a priori origins. It is assumed that people who answer a set of questions in a similar way comprise somewhat coherent social groupings or even "electorates," while a slight differentiation of answers stemming from age or sex differences is treated as a basis for speaking about "men" or "senior citizens" as if they really constituted a group in the sociological sense, or at least a distinguishable whole of some kind.

The seemingly obvious nature of the term "society" becomes particularly striking in the context of the crisis looming over sociology, as has been diagnosed by numerous authors. ${ }^{1}$ Among the reasons behind this crisis we can certainly identify changes in Western societies occurring since the 1970s, which entail, on the one hand, the "disintegration of previously existing social forms for example, the increasing fragility of such categories as class and social status, gender roles, family, neighborhood, etc.," while on the other - "the collapse of state-sanctioned normal biographies, frames of reference, role models" (Beck \& Beck-Gernsheim 1996, 24; emphasis preserved). However, the depth and range of the crisis are also conditioned by factors related to the development of sociology itself. After all, although it has accepted, from its very beginnings, the dual nature of social reality - originating in the actions of people yet appearing as external and objective to them; as something both limiting and directing their actions - sociology has nevertheless failed to come to terms with the problem of the passage from individual actions to resultant social entities. ${ }^{2}$ The dissolution of existing social forms and "the individualization of the society" - as diagnosed by Ulrich Beck and confirmed by most scholars of modernity - only caused the problem to resurface, thus questioning the theoretical usefulness of the very term "society."

In order to establish why the discipline which introduced the term "society" into common usage and adopted it as the fundamental object of research has ran

1 Interestingly, the claim that sociology is in crisis has been recurring quite regularly since the 1930s when Florian Znaniecki addressed the "deep crisis of sociology" (Znaniecki 2008, 29).

2 This issue has found fullest expression in the discipline's division into micro and macro sociology. 
into such trouble, we have to go back to its roots - the moment when sociology emerged as a distinct academic discipline.

\subsection{Classical views on the question of society}

For the founding fathers of sociology, society was certainly a key concept that had a par excellence theoretical character. It was an abstract construct encompassing all forms of interdependence among individuals, causing - firstly - that a certain group emerges, characterized by an internally integrated system of actions, which has a more or less permanent character, and secondly - that members of this group act according to certain rules (norms) of interaction which organize their cooperation and which they more or less consciously recognize. In this original interpretation, the society was simply a register of relations between individuals, an epiphenomenon of the mechanisms that coordinate their actions into some identifiable wholes. ${ }^{3}$

Thus, the proper subject of sociology would be specifically understood processes of socializing individual actions, i.e. directing them towards other people and supporting a certain pattern of interactions. It was of the utmost importance for classical sociologists to distinguish various historically determined forms of such processes. Already in his first book - The Division of Labor in Society (1893/2013), which contains all basic components of the sociological theory he developed later - Émile Durkheim introduces the distinction between mechanical and organic types of solidarity, viewing them as fundamentally different kinds of the social bonds that constitute the foundations of the traditional society (which he calls "segmentary") and the modern one in which he lived. Slightly earlier - in 1887 - Ferdinand Tönnies published his most famous study, in which he introduced two terms necessary to grasp a different mechanism of socializing (or of "mutual recognition"): Gemeinschaft and Gesellschaft, translated (respectively) as "community" and "society." Examination of the essence of social forms - although disregarding their historical conditioning - was also the main task of sociology according to Georg Simmel (1950), who considered "sociability" to be a pure, i.e. disinterested, form of socializing individual behavior.

3 The concept of coordination does not have a reconciliatory meaning here as it does not preclude conflict or change; it only indicates that the effects of an individual's actions are dependent on the others' actions. 
In contrast to Simmel, however, most authors of classical sociology argued that society cannot be defined by referring solely to its morphological features. ${ }^{4}$ This stemmed both from the multiplicity of real forms taken by such internally integrated communities, and from the fact that observable emergences of some group's morphological features can be entirely coincidental and not of a systemic character. ${ }^{5}$ The diversity of forms in which human society manifests itself - both in regard to the levels of sociality (small groups with direct ties vs. abstract state-communities) and in anthropological terms (tribal communities vs. modern society) - also indicated the necessity to theoretically embrace processes of societal variability or at least indicate their basis.

The interest in the processes of variation was additionally reinforced by the fact that classical sociology, which emerged as academic discipline at the beginning of the nineteenth century, has been developing in an era characterized by a rapid intensification of social changes, often accompanied by all kinds of painful side effects. These changes were all-encompassing, affecting not only the economy, politics and power structures but also such fundamental areas as demographics or strategies of family formation.

Economic changes consisted primarily in accelerated industrialization - a new mode of organization of production processes, which resulted chiefly in mass migration of villagers to cities. In consequence, the particularisms and regionalisms typical for feudal societies crumbled as cities became "melting pots" where new social categories emerged from a myriad of components.

As far as power structures are concerned, in most European countries the nineteenth century was basically the time when the process of breaking down the authority of individual power centers (controlled by aristocratic families) was brought to completion. Consequently, violence was "monopolized" by the increasingly influential nation state. A key role was played in this process by

4 Although they considered this type of research to be vital, they indicated the secondary nature of such scholarship in relation to the "content" of social phenomena. In his sociological project, Durkheim even distinguished a special area which he termed "social morphology." However, he firmly rejected the definition of sociology coined by Simmel because of its focus on researching social forms. Cf. Durkheim 1981.

5 In this context, Stanisław Ossowski, one of the best-known Polish sociologists, quotes an anecdote about a ticket clerk working in Warsaw's Teatr Wielki [Grand Theatre], who would sell tickets in such a way as to make the visitors' bald patches form a geometrical pattern visible from the balcony $(1967 b, 167)$. 
rational bureaucracy, which served as the basic means of organizing administrative structures and additionally functioned as a mechanism of standardizing actions by rooting them in a system of uniform regulations and by improving modes of control.

Politics also underwent intense transformations. Foundations of democracy clearly began to emerge in Europe, accompanied by ideologies, which acted as an instrument of mobilizing social support, crucial for representative democracy. The idea of "civic society" became widespread as a community of individuals engaged in "producing" the public good which, in turn, is managed by the state. Education became vital for nation states because it facilitated standardization of language, promoted writing and broadened common knowledge, all of which shape a sense of national identity and solidarity. Finally, the population itself and its quality became a "good" - hence the rise of health care for children in developed countries at the beginning of the nineteenth century.

Lastly, the first demographic transition occurred in the period in question. The nation state, which was in the process of establishing itself, faced a completely new situation. As Foucault observes (1988-1990), earlier forms of power had to cope with a scarcity of life. ${ }^{6}$ In contrast, the nineteenth century brought the leading European countries, especially the British Empire, an excess of life. New opportunities for obtaining the means of living through employment in the industry allowed young men from peasantry to marry before receiving their father's land. Consequently, the average marriage age lowered, and the total number of marriages increased as well (cf. Gillis 1974). Naturally, this also affected birth rates, which, in turn, led to mass unemployment in the next generation, especially in view of the transition from manufacturing to the factory system.

The above changes - including poverty and overcrowding in urban centers, the collapse of rural lifestyles and the rise of the new citizen who could have (and had) a say in public affairs - were visible to everybody. Experiencing this was accompanied by an acute sense of the collapse of the former social order: a ubiquitous anomie or "anxiety" born "because, certain of these [traditional] duties no longer being grounded on reality, a loosening of ties has occurred that can stop

6 This is aptly illustrated by the problem of workforce in serfdom economy. Due to high mortality rates, there was always a shortage of workers, although this was also affected by peasants fleeing from farms. Hence the restrictive rules regarding "marrying out" and other practices meant to guarantee an "abundance of life" matching the farmstead's needs. Cf. a broader discussion of these issues in Kula (1983). 
only when a new [moral] discipline has become established and consolidated itself" (Durkheim 2013, 318).

Thus, it was the accumulation of symptoms heralding deep changes, accompanied by indications of crisis, that brought sociology to life. These signs undermined the obviousness of directly experienced social life and therefore foregrounded the question about the source of social order. It is worthwhile to note here that the specific "denaturalization" of social life, which lies at the foundation of classical sociology, was a phenomenon extended over time, reaching as far back as to the Enlightenment when the problem of order - not only social order (cf. Szacki 2002, 82) - constituted the principal object of enquiry, or even further back to seventeenth-century social philosophy, which also asked about the source of rules regulating the behavior of individuals and consequently about the roots of social order. To Thomas Hobbes (1998) and Jean Jacques Rousseau (2002), who represent the two above traditions, the existence of societies was not something given by nature, but it demanded justification by referring to various types of social contract. Classical sociologists, in turn, redefine the problem into a question about the difference between old and new forms of collective life.

This question continually recurs in classical sociology, beginning with its forerunners Saint-Simon, Comte and Spencer, along with their naïve evolutionary schemas, through Durkheim, Tönnies and Marx, who referred to more historically grounded analyzes of the "transition to modernity," and ending with Max Weber, who explicitly ruled out the possibility of distinguishing any stages of social development, but nevertheless continued to revisit the question of social change, introducing a specific boundary between features of earlier forms of social life and those that emerged after the rise of capitalist economy. In other words, it was the final collapse of traditional forms of social order - triggered by processes which originated at the turn of the fifteenth and the sixteenth centuries (cf. Braudel 1983 \& 1992) - that gave rise to society as an object of sociological enquiry, revealing that underneath people's activities there exists something that organizes them into a certain whole and at the same time ensures its reproduction. It is this "something" that becomes named "society," displacing older or more specific notions of community, company or civic society.

Thus, one legitimate way to answer the question "what is society" could be to claim that society is a specific, historically distinguishable form of collective life, which began with the rise of capitalist economy and acquired its mature form in the nineteenth century. However, this answer would be incomplete because classical sociology was radically theoretical, i.e. it separated the observed phenomena from the hidden rules supposed to govern them, using the former as 
indicators of certain abstract intellectual constructs. ${ }^{7}$ Consequently, on the level of theory, the concept of society usually appears accompanied by another term specifying the dominant principle of socializing human actions.

Nonetheless, such an approach did not mean that the essence of that principle could be grasped just by the analysis of commonplace individual actions. This was particularly emphasized by Durkheim, who argued that "it is not the fact that they are general which can serve to characterize sociological phenomena" because " $[\mathrm{w}]$ hat constitutes social facts are the beliefs, tendencies and practices of the group taken collectively" (1982, 54; emphasis added). Despite the fact that individuals take action by orienting themselves with regard to certain socially constructed rules, "[n] one of these modes of acting and thinking are to be found wholly in the application made of them by individuals, since they can even exist without being applied at the time" (55). Social facts spread because they are social - not the other way round.

According to Durkheim, the hidden social reality is as objectively real as the physical one. Both are governed by rules that manifest themselves as coercion. We might, for example, consider social reality as entirely subjective until we feel the heavy arm of a policeman on our shoulder, or when our closest turn away from us with disgust. Behind social facts - life crystallized, for example, in the form of marriage rates - is a force of principles and rules, which are arbitrary (like grammar rules) but appear to individuals as something external and "self-evident," or as laws.

Despite all differences in literal phrasings, a similar approach to this "hidden reality" as the ultimate object of sociological research can be found in Weber. Although Jerzy Szacki rightly emphasizes that "Weber did not in fact need the concept of 'society'; this 'collective entity' was not the subject of his study" (2002, 467), Weber would argue that individual actions - as the basic area of enquiry for social sciences - ought to be analyzed by developing abstract "ideal types" that do not directly refer to reality. Moreover, he often argues that although the "ideas of values" that govern such actions have an arbitrary character because they are rooted in the choice of a meaningful world project, they are as objective for individuals as those encountered in natural sciences (Weber 1949). Thus, the

7 The most famous example is Durkheim's use of suicide rates as an indicator of social anomie. Distinguishing between observable phenomena and principles governing them - so clear in Durkheim - became an inspiration for Ferdinand de Saussure, contributing to the development of modern linguistics. The specific forms assumed by social life relate to the "social rules" exactly like parole (speech) relates to langue (language). 
aim of social sciences would be to reconstruct the project (i.e. "ideas of values") that lies at the foundation of human actions, with "ideal types" being the primary means of achieving such a goal.

It should also be noted that in classical theories the principle behind the constitution of society is also the principle that constitutes the individual. The ideal type of intentional and rational actions corresponds - according to Weber to homo economicus, on the one hand, and on the other, to the market-based modern order of the industrial society. Similarly, the ideal type of traditional actions corresponds to homo sociologicus, who prioritizes the replication of institutional patterns, and to the traditional order organized around a central image of the world. A similar correspondence can be traced in Durkheim, especially in his reflections on the change in the types of law. In this sense, the problem whether individuals constitute the society or vice versa is not really meaningful in classical sociology. Both options are secondary and derive from the dominant mode of sociality.

Therefore, we would say that the historically experienced radical change in the forms of socializing individual actions was the factor that gave life to sociology and its basic array of problems, at the same time making "society" the discipline's fundamental theoretical concept and the specific form of collective life born in the nineteenth century.

\subsection{Society as an object of enquiry in twentieth-century sociology}

If the first critical moment in the formation of sociology as a discipline was related to the emergence of modern society, the second one occurred at the turn of the 1930s and 1940s. ${ }^{8}$ It was at this point that sociology finally took its current shape, i.e. became a strictly scientific discipline that focuses on empirical research rather than theoretical speculations. This time, however, the shift occurred not in Europe but in the United States of America.

The second birth of sociology radically differed from the first one, primarily in terms of historical context. The sociology that developed in the USA in the 1930s did not have to struggle with answering the question: "What is society?" After a period of turbulent social changes, the American society was slowly becoming an increasingly self-evident notion in terms of its ontology, or - to put it more

8 Sociology suffered a period of stagnation in the 1920s. 
simply - it just was. ${ }^{9}$ The primary task of sociology would therefore consist in seeking, firstly, the most adequate notions that would help explain its lasting existence, and secondly - the most effective ways of examining it. ${ }^{10}$

The first task entailed a significant shift of emphasis in the discipline's field as defined in classical sociology: a move away from the question of how a specific type of society is produced towards the question of how this social entity, now conceived in abstract terms, is reproduced. It ought to be noted that both questions are usually answered in the same way: after all, the basic material used to erect the edifice of the society consists in individual actions. "The society exists only insofar as some people undertake some kind of activity in relation to each other," writes Piotr Sztompka $(2002,45)$. It seems difficult to undermine this definition. However, the shift of emphasis - from rules that allow speaking about specific types of society to processes of reproducing society as such radically changed the scope of sociology's fundamental problems. In the first case, researchers focused on the specificity of socializing processes, on identifying those structuring principles that could be treated as characteristics of a given type of society in the sense that they could be derived from cooperation processes taking place in different areas of individuals' life. In the second case, researchers began to be occupied with actions themselves, since their repetitiveness was supposed to be the mechanism responsible for reproducing structures underlying social order conceived in an abstract way.

This becomes apparent upon a comparison of theoretical programs formulated for sociology by Durkheim and Parsons. For the former, the axiomatic statement was that "[i]f there is to be true sociology, there must be certain phenomena produced in each society which are specifically caused by that society, which would not exist in the absence of that society and which are what they are because society is as it is" (1981, 1061; emphasis preserved). On the other hand, in a theoretical system developed half-century later in order to provide a

9 It is worthwhile to draw attention to the fact that this period had a completely different character in USA and Europe. The American society was born out of chaotic individual actions rather than by struggling with structures of the former social order. In this sense, it offered a completely different cognitive context when considered from the perspective of sociologists who described it.

10 As Hans Joas and Wolfgang Knöbl indicate in their discussion of Parsons's debate with the utilitarianists, "he is of the opinion that social order is an unquestionable fact, that there is order and that order is not a truly mysterious phenomenon. [...] Parsons asked which qualities of human agents might render social order possible" (2009, 30-31; emphasis preserved). 
basis for the practice of modern sociology, Talcott Parsons considered it equally obvious that these concepts need to refer to characteristics of individual actors; consequently, they need to be positioned in a broader frame of a general theory of action.

The findings of Parsons' The Structure of Social Action, first published in 1937, defined a lasting common ground for sociological discussions, providing a frame of reference for a specific consensus in research. Despite the fact that some of the book's specific claims have been subjected to criticism - and that the "normative" paradigm it set up faced competition from the "interpretive" one ${ }^{11}$ - in neither case was it really questioned whether Parsons was right in assuming that individual actions ought to be the point of departure for sociological considerations. The process of socializing and coordinating actions, leading to the formation of a higher-order entity, was explained in this framework not by indicating certain superior rules that would historically constitute a given society, but by analysing mechanisms that reference universalist assumptions lying at the foundation of a given theory of action. ${ }^{12}$

The individualization of modern sociology was certainly facilitated - especially in its early moments after the Second World War - by the development of quantitative research, which is till now considered by many scholars as the one and only scientific method of analyzing social phenomena. ${ }^{13}$ One could argue that post-war sociology was primarily an empirical discipline: the theoretical concepts it developed only slightly impacted research conducted in its

11 Cf. Wilson 1970.

12 Within the normative paradigm this mechanism was equated with the acquiring of social norms by individuals in the period of socialization, while in the interpretive one - with negotiating meanings in processes of interaction.

13 Naturally, it is also possible to enumerate (as is often done) other reasons for the process of individualizing social sciences. From a theoretical perspective, it was reinforced by the prevalence of neo-positivist philosophy in social sciences, favoring empiricism, as well as by the development of measuring and indexing theories. Moreover, pragmatism had a strong impact on the development of sociology in the USA. From a practical perspective, there was increasing demand for useful information regarding the opinions, needs and motivations of individuals, especially in industry (marketing research) and politics (election polls). Finally, from the perspective of ideology, the dominance of democratic regimes in Western countries (ones where sociology could develop after WWII) elevated the question of individual rights and equality in the face of law to the rank of a fundamental rule. None of these factors are accounted for here because the point of the present discussion is rather to underline differences in theoretical approaches developed by classical and modern sociology. 
framework, while the hidden patterns of social phenomena would be inferred from correlations and other statistical relations. What has become the fundamental datum of sociology is not even individuals' behavior but rather their opinions, which came to serve as the basis - more or less justified in theoretical terms - for drawing conclusions about the potential direction of social actions. ${ }^{14}$ In this way, individuals themselves became removed from their social environment because their "representative character" turned out to have merely a statistical meaning, one not reflecting the affiliation of study subjects to any broader community.

The rapid development of quantitative research that utilizes methods of statistical analysis imposed on sociologists a mode of thinking rooted in the categories of cause and effect. ${ }^{15}$ This stands in contrast to the approach of classical sociologists, who would conduct their analyzes employing the category of social processes. As Andrew Abbott argues (1992), the introduction of the new methodology involved making certain tacit assumptions, which can be traced in today's prevailing theoretical modes. Among these assumptions three play a particularly important role: the spatial and temporal independence of studied individuals; the nature of causality (treating specific explanatory variables as separate causes, disregarding their interactions); and finally, the consent to disregard the temporal order of changes occurring within particular explanatory variables (433-434). These assumptions not only reinforced the tendency to treat social phenomena as the total sum of individual actions but also basically precluded reflection on the relationship between the micro level (i.e. one of individual actions analyzed in terms of behavioral scripts, habits, lifestyles, values, interests etc.) and the macro level (i.e. one of social structures analyzed in terms of social rules, cultural patterns, institutions etc.). After all, as Abbott notes, the problem of this relationship is in itself a problem of time because it encompasses events

14 One classic example is the field of attitude research, the kind of work with which modern sociology is most often associated, and which boils down in practice to examining opinions of uncertain consistency and slight reference to both real actions (cf. Wicker 1969) and the theory of attitudes developed in social psychology (cf. Wojciszke 2002).

15 Although most methods of statistical analysis allow merely to ascertain the existence of an interdependence, the passage from the empirical finding that "level of education is correlated with income" to the conclusion that "higher education guarantees better income" is in many cases drawn almost automatically, if not by researchers then by the wider public. 
that occur in different tempos, might overlap, and have different implications for the course of the process they are a part of.

Undoubtedly, one of the consequences of such sociological practice was its division into two, unrelated currents already at an early stage of the discipline's formation after the Second World War. ${ }^{16}$ One current was focused on analyzing more general social structures, and assumed a chiefly theoretical and "speculative" character (as in theories of organization, social structure, culture etc.). The other current, more firmly grounded in empirical research, was concentrated on analyzing individual convictions, values and attitudes, seeing them as the main variables explaining individual actions. ${ }^{17}$ This division was accompanied by the increasing partitioning of sociology's field into specific areas of social life examined by different kinds of "sociologies": sociology of family, of religion, of culture, of politics etc. They evolved in separation, developing unrelated explanatory concepts and distinct modes of organizing professional life, but rarely communicating with each other. Such specialization occurred at the cost of a more general reflection on society - the concept that transformed from one having many theoretical dimensions into a descriptive self-evident category.

Thus, a distanced perspective on the changes in post-war sociology reveals one peculiarity: at the basis of its development there was a secondary de-theorization of the discipline's fundamental concept - that of society. It did not matter much until the entity behind this term retained the basic characteristics of a particular form whose emergence gave rise to classical sociology and constituted the empirical basis for the later developed methods of studying social phenomena. However, along with the intensification of changes in contemporary society it became apparent how risky it may be to allow for the widening of the gap between implicit assumptions regarding the nature of the society, conceived in abstract terms, and processes or phenomena occurring within one of its historical form.

Naturally, the de-theorization of the concept of society did not uproot theory from sociology. Instead, it gave theorizing a specific character. On the one hand, the "obvious" character of the very concept of society bred a temptation to treat processes occurring in it comprehensively, which found expression in attempts

16 They have their counterparts in two fundamental works by Parsons, who developed not only the above-mentioned theory of social action, but also one of social system (1951).

17 It is worthwhile to recall here that the concept of attitude was introduced by Thomas and Znaniecki (1918) to denote individual counterparts of social values. It was only with the development of empirical sociological research that these two concepts began to be treated as describing differentiation of individual beliefs, needs and feelings. 
to develop a "general social theory," or "Grand Theory," as C. Wright Mills called it in 1959 (2000). These attempts usually took the shape of elaborate conceptual formulas that would derive the existence of social structures from the most fundamental idea of social action. ${ }^{18}$ On the other hand, the highly abstract character of such formulas, as well as their diminished usefulness in explaining particular social phenomena, enforced in some ways the parallel development of so-called mid-range theories, constructed usually around single concepts, rather freely derived from the tradition of social thought or constructed ad hoc to satisfy the needs of a specific theory.

The liberation of theory from broader reflection on the nature of society left, at the turn of the 1960s and 1970s, deep cracks in the understanding of the character and goal of social sciences, which had been shaped by Parsons' works. Giddens argues that "the loss of the center ground formerly occupied by the orthodox consensus has seemingly left social theory in a hopeless disarray," pointing out it has been torn apart by "the babble of rival theoretical voices" (1986, xvi). As he emphasizes, most "novel" theoretical approaches developed at the time had rather old roots and were merely revisiting those ideas or views that had been abandoned earlier or functioned at the margin of sociological mainstream. ${ }^{19}$

Drawing from the tradition of social thought could hardly be objected. However, one may raise reservations concerning the way in which the canon was utilized because a significant portion of the "novel" approaches - despite being invigorating and refreshing in comparison with traditional directions taken in sociology - turned out, with the passage of time, to be as useless in analyzing the rapidly accelerating processes of social change as the "old" approaches. ${ }^{20}$ Thus, the emergence of new positions only reinforced the sense of inconsistency marring sociological explanations and thereby deepened the feeling of "cognitive

18 A classic example of this is of course the theory developed by Parsons, while its contemporary counterpart would be that of Giddens (1986).

19 Giddens points out, firstly, the emergence of voices neglected by Parsons, which foregrounded the phenomena that some of his critics "saw primary in Marx - class division, conflict and power" (xiv), and secondly - the fact that the American "market" saw the rise of new theoretical schools born in Europe, such as phenomenology and the critical theory of the Frankfurt School (xv).

20 This also concerns Giddens's own theory of structuration. Although his work has been widely recognized and enjoys respect due to its comprehensive scope and elaborate theoretical apparatus, it seems that it has not really impacted actual research practice. 
confusion" (Misztal 2000) which sociologists have experienced on a daily basis in the face of an increasing number of new social phenomena.

Of course, this does not mean that sociology ignored the emergence of those new phenomena. However, their treatment was strongly conditioned by the "division of labor" developed earlier. Thus, transformations of society were registered on the empirical level either as changes of more general social structures ("subsystems," to employ Parsons' terminology), or as sequences of changes in individual traits such as aspirations, values or attitudes.

The clearest example of an approach that follows the first model is the concept of "post-industrial society" popularized by Daniel Bell (1973). In the 1970s and 1980s, many researchers employed this idea in their efforts to analyze social transformations. For Bell himself, the key characteristic of post-industrial society was visible in the changes in the USA's social structure, which were related to the increase in the number of people employed in the services sector. These changes were the outcome of the passage from technologies based on using various forms of energy to ones rooted in data processing, a move facilitated by "theoretical knowledge." According to Bell, this process entailed a change in factors regulating the society's functioning because the "game against fabricated nature" (which determined the basic design of the industrial society) began to change into the "game between people," who became engaged in creating, gathering and processing information. The basis of the fundamental social conflict also underwent a vital transformation: "If the struggle between capitalist and worker, in the locus of the factory, was the hallmark of industrial society, the clash between the professional and the populace, in the organization and in the community, is the hallmark of conflict in the post-industrial society" $(1973,129)$.

Introducing the concept of post-industrial society, Bell often emphasized that out of society's three dimensions that make it possible to describe it - namely social structure, politics and culture - he would limit his analyzes only to structural transformations and their consequences for the realm of politics. He certainly had the right to do so. However, it remains rather unclear why changes in exactly these dimensions would justify speaking about a new type of society. While he has already mentioned in The Coming of Post-Industrial Society that the rise of post-industrial society is necessarily correlated with the growing disjunction between the spheres of economy and culture (1973), ${ }^{21}$ later focusing on this disjunction directly in the Cultural Contradictions of Capitalism (1976), where

21 Cf. especially the chapters "The Dimensions of Post-Industrial Society" and "Coda: An Agenda for the Future." 
he underscores the role played in its creation by modernity, which was "driven by the self-infinitizing spirit of the radical self" (xxix), it remains unknown what would link changes occurring at the level of macro structures with changes in culture.

The lack of connection between macro and micro levels - i.e. between more general social structures and individual behavior - was also acutely felt in research focused on registering changes in attitudes, values and views of individuals. ${ }^{22}$ It is quite telling that concepts synthesizing such changes, e.g. consumerism, reflexivity or individualism, were used in turn to explain transformations of entire societies. This is best illustrated by the career of the concept of individualization. It has been understood, on the one hand, as a process involving individual striving for self-fulfillment and development of one's potential, and on the other - as the sum of transformations occurring in basic areas of social life in contemporary society, a perspective popularized by Beck (1992).

The concept of "individualized society" - a contradiction in itself - invites a series of questions, casting doubt on both currently dominant research practices and their solutions to specific social problems. Beck himself draws attention to this by asking: "Is it possible, at all, for a society in the drifting sand of individualization to be registered statistically and analyzed sociologically? Is there any remaining basic unit of the social, whether the household, the family, or the commune? How could such units be defined and made operational? How should the various political spheres - for example local politics, traffic policy, environmental policy, family policy, welfare policy - react to the diversification and transitoriness of needs and situations? How must social work (and its educational content) change when poverty is divided up and, as it were, distributed laterally among biographies? ${ }^{23}$ What architecture, what spatial planning, what

22 This kind of perspective forced the explanation of attitudes, values and convictions mainly in psycho-social or cultural terms. As a result, culture (defined as a system of values) has also basically changed its character: it was no longer a systemic concept, but rather one that describes attributes of individuals. It is therefore a small wonder that perhaps the only sociological theory attempting to account for contemporary changes is now the one formulated by Ronald Inglehart (1990) - the theory of transition from material to post-material values.

23 Beck refers here to the change he widely commented on (cf. Beck 1992), which consists in the passage from the "vertical" distribution of poverty in the society (affluent upper and middle classes, and poor lower classes) to the situation in which most individuals have their chance, or are rather exposed to the risk of becoming impoverished at some point in their life. 
educational planning does a society need under the pressure of individualization? Has the end come for the big parties and the big associations, or are they just starting a new stage of their history?" The primary question, however, is this: "[I]s it still at all possible to integrate highly individualized society?" (Beck \& Beck-Gernsheim 1996, 42-43).

Today's sociology leaves most of these and similar questions unanswered, focusing in its analyzes of ongoing changes on documenting various peculiarities characteristic of contemporary social life. One could even say that although in recent years most sociologists' basic preoccupation was with finding new qualities that would grant original status to contemporary patterns of social actions, these qualities are in fact usually described in isolation from systemic features of society as such or treated as a continuation or a distillate of attributes characterizing modern society. Furthermore, analyzes of social structures - even though some of their findings may have suggested that we might deal with a change of society's fundamental form - have not yet offered any theoretical synthesis or even provided ground for agreement among the various positions, which could serve as the basis for a new consensus. ${ }^{24}$ Although the conviction that "the kind of society analyzed by Marx, Weber and Durkheim, the kind of society inhabited by most westerners for the past century and a half, is no more" (Kumar 1995, 2-3) is rather widespread, it has almost no influence on either the conceptual apparatus of contemporary sociology or its research-related practices. Maybe, because it is highly vague what other type of society displaced the one examined by classical sociologists.

Contemporary sociology steers clear of the problem posed by the Great Change, i.e. the systemic transformation that is now altering the basic qualities of society as a historically given form of organizing human activities. Even those like Giddens or Beck - who have adopted a more comprehensive approach in their accounts of ongoing changes - prefer to speak of "late modernity" or "second modernity," avoiding direct answers to questions about the extent to which those "late" or "second" versions of the modern society actually differ

24 One attempt at offering such a synthesis was made by Manuel Castells in the trilogy The Information Age (2001a, 2002, 2001b). However, the factual density of his analyzes as well as his avoidance of "excessive theorization" make any simple reconstruction of the author's conceptual apparatus problematic (cf. Stalder 2008). His only concept that became widespread is the idea of "network society" (although he popularized it, it is most often used in ways that contradict his theory), whereas the notion of "informationalism," which is crucial in Castells's theoretical system, has not attracted broader attention among researchers. 
from the "early" or "original" rules that organized the modern type of social order. As Zygmunt Bauman emphatically puts it, "postmodern sociology is distinguished by avoiding confrontation with postmodernity as a certain form of social reality, as a new departure set apart by new attributes" $(2003,41)$. It is a "mimetic representation of the postmodern condition" (42) rather than an attempt at describing social reality from outside - an effort which would involve a more or less disciplined search for those characteristics that are specific for the new type of social organization.

Without a doubt, one of the basic reasons for this state of affairs is the fact that contemporary sociology does not have any concept of society as an entity that assumes various forms. Despite the collapse of the "orthodox consensus" grounded in works by Parsons, questions posed in sociology's mainstream are still rooted in a reified vision of society as a system comprised by basically unchanging structures that would constitute its essence, thus creating a framework for individual actions, although these actions may not necessarily follow the logic of those structures. Given such an approach, what disappears from the view of researchers is the systemic character of historically shaped types of social order. The consequences of this are twofold. First, theoretical reflection would rather focus on developing conceptual formulas isolated from the historical variability of actually existing social systems. ${ }^{25}$ Second, empirical research would be in turn subordinated to separate, objectified structures or patterns of individual behavior, analyzed without accounting for their systemic entanglement.

At the same time, however, it is precisely those two features - systemic character and historicity - that underlie the concept of society as a specific, nineteenth-century form of socializing individual actions, which constituted the object of study for classical sociology. Parsons' transposition of this historically given form of sociality into a timeless entity called "society" occurred in defiance of assumptions made in classical sociology. ${ }^{26}$ The same happened to the

25 Anthony Giddens (1986) attempts to overcome this limitation by introducing the concept of "space-time continuum," which performs a vital role in distinguishing specific types of systems (societies). However, this occurs at the cost of blurring the "systemic character" of society. Jerzy Szacki remarks that such a character is "relative through and through" for Giddens, and in the case of contemporary societies is, according to him, overestimated by sociologists who "seem oblivious to the fact that the 'systemic character' [of society] is rooted in administrative unification rather than in the 'infrastructure of social order" "(Szacki 2002, 884).

26 As Durkheim claims, "it is not a matter of considering society at a given moment in time, frozen in an abstraction, but rather of analyzing and explaining its formation. [...] Structure itself is revealed in society's becoming and one can only illuminate it on 
analytical distinction, introduced in later scholarly practice, between individual behavior and the structures it creates. The difficulties encountered by contemporary sociology while attempting to grasp processes of social change, to answer the question posed by Beck ("What, if anything, integrates today's society?") and to identify the main mechanisms transforming social life today have their roots in the failure to acknowledge the historical and systemic character of society. This is why the crisis of society we experience these days is at the same time a crisis of sociology.

Thus, let us return to the period before contemporary sociology fell into this conceptual trap, and explain what the term "society" actually meant when it was used as a theoretical concept and a cornerstone of investigations carried out in classical sociology.

\subsection{Society as a theoretical concept}

Reflecting on contemporary social changes, Norbert Elias draws attention to the relationship between the meaning of certain abstract concepts and the crucial problems of a given epoch. In his view, the very term "abstract" is misleading because such concepts are not created when a researcher isolates some more general qualities from particular cases. They are rather forged in the process of synthesizing "many common elements that made a new, previously unknown entity accessible to communication, raised it into the light of understanding" (2001, 159), thereby reaching a broader community. The development of concepts would thus be the outcome of a long social process, during which the changing conditions of living produce new experiences that are - for some reason - crucial for a given society, and whose particular components are synthesized by introducing new words or changing the meaning of ones already in use. ${ }^{27}$

condition of not losing sight of this process of becoming. It is constantly becoming and changing; it is life having crystallized to a degree; and to distinguish it from the life from which it derives or the life that determines it amounts to dissociating inseparable things" (1981, 1060).

27 It is worthwhile to recall, as a side note, that Elias's position undermines the legitimacy of both inductive and deductive scientific models by ascribing a fundamental role to social praxis, both in the narrow sense, i.e. in reference to the community of scholars, and more widely, i.e. in reference to the concept of society as a whole. According to Elias, it is the amassing of new experiences that gives rise to new concepts (both in scientific discourse and everyday language); these experiences are derivative of actions (regardless whether they are scientific experiments or forms of everyday behavior) and 
Looking from this perspective at events preceding the birth of sociology, it could be stated that the new aspect of social existence, giving rise to the term "society" in its contemporary understanding, consisted in the ever more overwhelming experience of mutual bonds tying individuals into wholes that exceed the sum of direct contacts, and of the resulting dependence on the actions of other, usually unknown people. The consequences of this experience have been succinctly summarized by Georg Simmel, who argued that "the recognition that man in his whole nature and all his manifestations is determined by the circumstance of living in interaction with other men, is bound to lead to a new viewpoint that must make itself felt in all so-called human studies" (1950, 12; emphasis added).

To realize the extent of the novelty inherent in this new viewpoint it suffices to recall that the intellectual currents preceding sociology were radically individualistic. Both concepts rooted in the Enlightenment and ones developed on their basis in liberalism placed individuals in the center of attention, making them the fundamental object of analysis. "Liberalism's point of departure was to imagine a sovereign individual naturally endowed with certain rights, which cannot be waived or invalidated through arbitrary political decisions made in the name of a supposed general good" (Szacki 2002, 148). The freedom of individuals, guided in their actions by natural instincts, feelings or selfish interests, was considered to be that which defines the framework for explaining social processes, and simultaneously constitutes the greatest value, therefore obliging to protect it from any political designs.

Individualistic accents in descriptions of society in that period can be also traced in conservative thought developed at the turn of the eighteenth and the nineteenth centuries, which was critical of the Enlightenment and liberal traditions. It constituted a reaction to the social transformations that originated in industrialization and the democratic revolution. "Conservatives considered the fall of the former system as dangerous: if not as the 'dissolution of the society' then at least as a hazardous disturbing of its foundations, consisting mainly in severing traditional ties between people. The post-revolutionary society appeared to its adversaries as a formless assembly of selfish individuals, all of whom pursue their own interests without minding their neighbors"28 (Szacki 2002, 142).

cannot be communicated by employing the conceptual apparatus used before. This has also informed the emergence of a host of new concepts in contemporary physics.

28 There is a striking resemblance between this account and contemporary diagnoses of the "crisis" or "individualization" of society. This question is addressed later in this book. 
In the context of the two above intellectual positions, the perspective offered by the rising discipline of sociology in fact appears as radically distinct. First, classical sociologists neither defied the emerging social reality, nor treated it as a departure from the "natural order" or the arrival of some "chaotic state." They rather considered it to be a new form of social order, characterized by properties different from those that defined previous ones. The specific "reconciliation" with the effects of processes leading to change, which are extended over longer periods of time, is clearly discernible in Durkheim, who notes in the introduction to The Division of Labor in Society that "[w]e can no longer be under any illusion about the trends in modern industry. It involves increasingly powerful mechanisms, large-scale groupings of power and capital, and consequently an extreme division of labor" (2013, 33; emphasis added). Tönnies puts a basically similar thought in different words when he points to the necessity of adopting an "unbiased and theoretically rigorous manner" in analyzing " $[w]$ hat we experience as the 'present." " Furthermore, he bemoans the fact that "even a learned and critical public does not as a rule want to be told the view of an author about how things are, how they came about, and how they will turn out; they would rather hear how he thinks they should be" (2001, 9-10; emphasis preserved).

Second, classical sociologists held no illusions concerning free will as the chief characteristic of individuals, which makes it possible to explain their actions. The realities of the end of the nineteenth century did not leave much room for such a misconception since the individual had already become subject to the homogenizing forces of the increasingly powerful state, embedded into nation, class, factory or other collectives assembled on various levels of social life's organization, which all share one property, i.e. the ability to impose certain limits on individuals, subjecting them to specific regulations. "The individual," Simmel argues, "is thus doubly oppressed by the standards of society: he may not transcend them either in a more general [i.e. specific to all humankinds], or in a more individual direction" $(1950,64)$. Durkheim, on the other hand, notices in the "Preface to the First Edition" of The Division of Labor in Society that although "science presupposes the entire freedom of mind," it becomes paramount to "rid ourselves of those ways of perceiving and judging that long habit has implanted within us" $(2013,6)$. In this sense - as has been rightly noted by Zygmunt Bauman - the kind of sociology that focuses on explaining social coercion would become primarily a "science of unfreedom" $(2010,1)$.

Third, this has also involved a shift of focus from the individual to the collective. It was facilitated not only by an increased awareness of the rising role of collective actors in nineteenth-century society (e.g. the "third estate," the emerging labor movement, capitalists as a group of shared interests, and even 
the mob) but also by the conviction that the society itself is a specific synthesis of "lower-degree" collectives. Consequently, everything that occurs within particular collectives would have repercussions for the functioning of the higher whole. When Durkheim writes about the traditional society, which is assembled by way of mechanical solidarity, he also adds that "so long as society is made up of segments what occurs in one of these has less chance of having repercussions upon the others, the stronger the segmentary organization"; however, as Durkheim continues, referring to the modern society organized in accordance to principles of organic solidarity, "[a]s a result of their [the organs'] mutual dependence, what infects one infects others, and thus any serious change assumes a general interest" $(2013,175)$.

The above three context-shaped properties of the mode of thinking developed in classical sociology can be also found in twentieth-century sociology. It could be even said that for a long time they have defined the discipline's scope of interest by emphasizing the specific opposition between the individual and the collective; in this framework individuals would be primarily an object of social influences, while collectives would be the basic components of society. As Zygmunt Bauman put it, "concepts like class, power, domination, authority, socialization, ideology, culture and education organized the sociological map of the human world. What all these and similar concepts had in common was the idea of an external pressure which sets limits to individual will or interferes with the actual (as distinct from intended) action" $(1988,5)$. This "sociological map of the human world" also imposed a mode of articulating new experiences, which had no counterpart in nineteenth-century society, and which resulted from the increased pace of social changes in the second half of the twentieth century. After all, the discovering of individual agency, a process marking all attempts at departure from the orthodox consensus of post-war sociology, occurred in the sphere of those notions of the society that have been shaped by the fundamental nineteenth-century opposition between individual freedom and "unfreedom."

We should stress, however, that the new "viewpoint" which would define the specific nature of the sociological approach had a much broader character in classical sociology. This has been most clearly articulated by Georg Simmel, who wrote that "[i]t is only a superficial attachment to linguistic usage (a usage quite adequate for daily practice) which makes us want to reserve the term 'society' for permanent interactions only. More specifically, the interactions we have in mind when we talk about 'society' are crystallized as definable, consistent structures such as the state and the family, the guild and the church, social classes and organizations based on common interests. But in addition to these, there exists an immeasurable number of less conspicuous forms of relationship and kinds of 
interaction. Taken singly, they may appear negligible. But since in actuality they are inserted into the comprehensive and, as it were, official social formations, they alone produce society as we know it" (1950, 9; emphasis preserved).

Historians of social thought have accorded Simmel the status of an outsider in classical sociology. They focused on his "formalism" and would only go so far as to stress that his insistence on studying those "less official" forms of interpersonal relations gave rise to micro sociology, which was developed later. Such a reading of Simmel was strongly influenced by Durkheim's polemic and the tradition of the history of social thought, which evolved towards a "taxonomy of genres," focused on identifying similarities and differences (established according to obscure criteria) between thinkers. However, it is the "ontological" dimension of Simmel's work that is of greater importance for us than the "systematic" one, because we aim at establishing what lies at the foundation of society as a theoretical concept that has emerged at a specific moment in the development of social reflection and come to constitute the instrument of articulating vital social experiences.

From this perspective, it becomes crucial that Simmel has drawn attention to the existence of a more - so to speak - "elementary" level of social phenomena than the one analyzed by Durkheim (and by contemporary sociology, which followed in the latter's footsteps). It would consist of "immediate reactions that occur among men" and create social ties between them. Simmel claims that "society, as its life is constantly being realized, always signifies that individuals are connected by mutual influence and determination" (1950, 10; emphasis added). These connections may be more or less lasting, but they in fact determine the nature of social life. Society would consequently be "something functional, something individuals do and suffer." Thus, "true to this fundamental character of it, one should properly speak, not of society [Gesellschaft], but sociation [Vergesellschaftung]"29 (10).

Let us dwell on this point because it may lead, especially when lifted from its context, to the conclusion (often drawn in the past) that "interaction" is a central concept in Simmel's theory (cf. Szacki 2002, 454). This would be perfectly sound had it not been for the fact that Simmel's "interactionism" fundamentally

29 The term "sociation" coined by the English translator for Simmel's concept of Vergesellschaftung did not catch on in sociology. It is one of the reason why in this book we use the phrase "socializing processes" for the description of such interactions that generate relatively stable patterns of connections between individuals and in consequence, stabilize the forms of coordination of their actions. 
differs from contemporary theories that employ this concept. The difference lies primarily in the fact that for Simmel the insistence on the significance of mutual interactions occurring between individuals does not constitute a goal in itself and does not lead to the conclusion that it is interactions themselves or individual actions composing them that ought to constitute the subject of sociological research. On the contrary, he makes it clear that the rules of human behavior should interest sociology only "insofar as they form groups and are determined by their group existence because of interaction" (1950, 11; emphasis added).

In this light, it becomes possible to offer a different interpretation of one passage from Simmel, which is often cited as evidence of his "interactionist inclination": "Sociation continuously emerges and ceases and emerges again. Even where its eternal flux and pulsation are not sufficiently strong to form organization proper, they link individuals together. [...] the whole gamut of relations that play from one person to another and that may be momentary or permanent, conscious or unconscious, ephemeral or of grave consequences [...], all these incessantly tie men together. Here are the interactions among the atoms of society. They account for all the toughness and elasticity, all the color and consistency of social life, that is so striking and yet so mysterious" (1950, 9-10). There is neither any suggestion here that the study of more lasting forms of these ties ought to be abandoned, nor any objection to the analyzes of those historical forms of "sociation" prevalent in a given period, which were conducted by Durkheim or Tönnies. What we find in this quotation is rather a warning against identifying society with its particular nineteenth-century institutional form, and an emphasis on the processual nature of social life.

For Simmel, "[t]he large systems and super-individual organizations that customarily come to mind when we think of society are nothing but immediate interactions that occur among men constantly, every minute, but that have become crystallized as permanent fields, as autonomous phenomena. As they crystallize, they attain their own existence and their own laws, and may even confront or oppose spontaneous interaction itself" (1950, 10; emphasis added). However, this does not mean that they should last indefinitely. Socially created, they depend on connections between individuals, which alone uphold such systems. That is why they may also change along with major transformations of the underlying connections. Thus, if an identified type of shapes and structures is destroyed, it does not mean that society is dissolved too. As a theoretical concept, it is ultimately nothing more than a historically dominant form of social ties, which exist as long as individuals enter into contact.

We have devoted a lot of space to the reconstruction of Simmel's thought because from our perspective it offers the kind of theoretical account of the nature 
of society that is both general and open-ended, facilitating analyzes of society's contemporary transformations and simultaneously allowing to incorporate most findings of classical sociologists and their successors. Let us close this chapter with a list of its basic elements. First, on the most abstract level, society can be regarded as a form of sociality, prevalent in a given historical period, rooted in the processes of socializing individual actions. Second, such socializing processes always take place due to mutual interactions between individuals, and result in the formation of ties among them on various levels of social reality. Third, the emergence of these ties leads to the creation of social groups. Fourth, in the course of individual actions such groups acquire a degree of autonomy, which, in turn, allows them to affect the behavior of individuals by channeling it into more permanent forms, which contributes to the reproduction of the dominant form of sociality. Finally, the entire process has a continuous character, which means that under certain conditions mutual interactions between individuals can lead to the emergence of new types of ties, thus giving birth to a new form of society.

This theoretical perspective shall inform our further analyzes. 


\title{
CHAPTER TWO. MECHANISMS OF SOCIALIZING PROCESSES
}

\author{
Research undertaken in this study was born out of a \\ deep conviction that only now, in our times, thanks to \\ discoveries made in human sciences and accumulated \\ during the last century, it has become possible to verify the \\ principle formulated by Durkheim.
}

GEORGES GUILLE-ESCURET, Le décalage humain

Consciousness develops from this continuous interaction in which biology organizes experience and experience organizes biology.

STANLEY I. GREENSPAN \& BERYL L. BENDERLY, The Growth of the Mind

Human beings are not wild animals. They are domesticated by the work of mothers, psychiatrists, priests, policemen, teachers, and so on.

ROM HARRÉ, Social Being

The conviction about the social nature of human beings is one of those fundamental assumptions in sociology that are usually not even mentioned in contemporary works in this field. At best, this issue is addressed in introductory chapters of handbooks, where proof of this claim is usually said to be provided by the example of "wild children" - one that is quite old and has been repeated ad nauseam. ${ }^{30}$ The example shows that, because these children have grown up outside a social environment from their early youth, they have not mastered

30 The fate of probably the most fundamental axiom in sociology - which lies at the root of its claim to be a "unique" area in research, as determined by the fact that it is people's growing up among others, as well as their inherent dependence on them and on their actions, that creates humanity - is probably a perfect illustration of the natural process of the banalization of concepts. With the passage of time they are becoming increasingly "empty" due to the fading of their original meanings and of the intentions that have accompanied their use. The fact that sociology uses a natural language, only trying to modify and enrich it to make its concepts more precise, certainly facilitates and accelerates this "banalization." 
any of the typical human competences; moreover, they would be incapable of developing them fully even after being introduced to society. It is deemed equally obvious that the "humanization" of people as representatives of a particular biological species occurs through culture, i.e. a historically developed set of practices, beliefs and norms, transferred by way of socialization processes and lending human actions their specific character. More pedantic authors mention two human-specific competences - the ability to use language and tools - as something that distinguishes people from other animals. The terms homo symbolicum and homo faber (as well as their many variations) are most frequently used in social sciences to capture the difference between man and animal, which is viewed as something innate, i.e. grounded in certain biological properties of human brain, making humanity superior to other species.

The deep conviction about the uniqueness of humanity does not seem to be affected by research done in natural sciences, which proves that the use of tools is quite widespread in the animal kingdom, especially among primates, though these tools may not be as sophisticated as those developed with the help of modern technology. Moreover, knowledge about their use is transmitted from generation to generation by teaching. Most species have also communication systems, some of which are complex to the point of being labelled as languages. ${ }^{31}$ Although such findings blur the boundary between the human and the animal, they are usually ignored by sociologists, or ascribed to mechanisms rooted in instinct, which are believed to differentiate animal behavior from cultural mechanisms that lie at the foundation of human actions. ${ }^{32}$

It is not our aim to question the existence of some boundary that would make it possible to differentiate between the behavior of people and other species. The point is rather to indicate that at least some definitions of this

31 A detailed account of long-term research on the language of vervet monkeys can be found in Jared Diamond (1991, Chapter Eight). Its most important conclusion seems to be the one that their language contains at least ten distinct "words" - sounds tied to particular referents (leopard, eagle, snake, etc.) - and that it is intentional, i.e. there is an intention at the foundation of its use, for example a desire to warn the group. Both of these features were traditionally believed to be specific only to human communication.

32 It is worth emphasizing that at some point a shift of accent occurred in sociology: "social being" was replaced with "cultural being," while social environment - entailing that in order to develop specifically human skills it is necessary to grow up among other people - was supplanted by cultural environment, which involves being surrounded by symbols, values and cultural artefacts. 
boundary - traditionally referred to by sociologists in attempts to render the meaning of the concept of sociality - cease to be valid, and that in the light of results provided by other human sciences this fundamental sociological notion begins to lose its self-evident character. Moreover, its understanding sometimes emerges as blatantly false, which crucially endangers the legitimacy of claims made by this discipline. The intensification of this process is certainly related to the phenomenon described in the previous chapter, i.e. the de-theorization of the concept of society. However, it was also influenced by some earlier predicaments that sociology ran into when trying to determine its identity (cf. Mills 2000), the side effect of which was a basically programmatic refusal to encroach on the territories developed earlier by other disciplines. ${ }^{33}$

Such problems became apparent already at the very dawn of sociology. It was Durkheim who claimed that "every sociological problem assumes a thorough knowledge of all the special sciences that Simmel would wish to put outside sociology but without which it cannot exist" (1981, 1058; emphasis added). Durkheim recognized the difference introduced by sociology not in its different "field of study" or even methods, but rather in its specific approach to social facts - one based on the premise that they would be "studied like things." ${ }^{34} \mathrm{He}$ believed that sociology is, in its very nature, an interdisciplinary science - "he did not believe," Jerzy Szacki argues, "in any meaningful separation of sociology from other social sciences, or in the possibility of practicing it independently of data provided by other disciplines, especially history" $(1965,101)$. According to many contemporary scholars, however, sociology has become a discipline whose cumulative scientific output allows to independently develop research projects or theoretical concepts without the necessity to consult findings of other sciences. Needless to

33 This is pointed out by David Stark, who writes that in "developing his grand designs for sociology," Parsons "made overt signals to his colleagues in the Economics Department at Harvard alerting them to his ambitious plans and assuring them that he had no designs on their terrain" (2000, 2; emphasis preserved). This gave rise to what came to be known as "Parsons's Pact," according to which economists are supposed to study value, while sociologists - values (cf. also Camic 1987). Similarly, it is possible to speak of a "Parsons-Kroeber Pact," which assigns the study of social relations to sociology, and the analysis of the meaning of social actions - to anthropology (cf. Parsons \& Kroeber 1958).

34 This claim demands that social facts be treated as part of objective reality, which exists independently of individual consciousness. Therefore, the social world would be as objective as that of nature, with one key difference being that the former's objectivity stems from moral coercion, not from physical necessity. 
say, such isolation may also lead to the solidification of errors and can preclude apt usage of findings from other areas of human sciences.

Georges Guille-Escuret (1994) has pointed to, more universal reasons for the lack of communication between particular areas of human sciences. In his view, "the crucial obstacle in developing an interdisciplinary perspective that would combine natural and social sciences manifests in the persistent tendency to erect artificial barriers between dynamic, coherent wholes [cohérences mouvants], subjected by individual sciences to conceptual formalization, as in the case of population, ecosystem, culture, society etc. The desire to position every such category firmly on a particular 'storey' of reality leads to the rupture of its relationships with many others that also shape it, and to decreeing it forbidden to establish communication with other coherent wholes described by other sciences" $(1994,18)$. As a result of such "separatist" attitudes, knowledge about humanity has become dismembered, which has also led to the division of the human being into a series of "clones" that fit neither each other nor the data gathered by disciplines taking the human as their subject, and especially the explanations they propose. ${ }^{35}$

This is particularly clear in the case of such concepts as socializing processes, whose very nature brings together themes deliberated in various areas of social sciences. Analyzed in the context of anthropogenesis, it constitutes an object of interest for biology and evolutionary anthropology, though it is also being discussed by philosophers, historians of technology and even specialists in cave paintings. As a theoretical notion, it is employed in formal models of the theory of evolution, games theory, and even within certain currents in economics. In the individual dimension, the process of socializing has been long considered the domain of sociology and social psychology, which have focused on analyzing socialization mechanisms and interpreted them in terms of learning or training necessary to become a member of society. However, since the 1950s, interest in researching socialization processes began to dwindle in these two disciplines due to a number of factors, including especially the increasing criticism of the normative paradigm founded in Parsons' works. ${ }^{36}$

35 This, in turn, strengthens the initial isolationist tendencies: the models and findings of other human-focused disciplines seem to be so radically contradictory to "our" approach that the only solution is to reject them by indicating that they do not account for certain fundamental characteristics or phenomena: human irrationality, altruism, freedom, or autonomy.

36 One of the first critics to directly attack Parsons's view of socialization as a mechanism of reproducing social order was Denis H. Wrong (1961). At the beginning of the 1960s, he published his best-known article on the "oversocialized conception of man," 
One might have the impression that even if modern versions of sociology and social psychology raise the issue of socializing processes, it appears only as a magical formula that enables one to focus attention on the present exclusively, without bothering about the past. Socialization of human beings merely happened at some point, just like society simply is. From that moment on, human beings boast unique qualities, regardless whether they are viewed en masse as a species, or considered from the perspective of their individual biographies. Those qualities elevate them above the animal kingdom, in which there is no other species that would possess the crucial and exceptional ability to think.

However, if we wish to return to original intuitions offered by classical sociology in our effort to define society as the dominant form of socializing processes, these processes demand closer scrutiny.

\subsection{Sociality in the perspective of anthropo- and sociogenesis}

Regardless of how detailed its definition may be, the concept of sociality involves the idea of a certain change brought about in the functioning of individuals by the very fact that they lead their lives among other people. Individuals become socialized only by being included in a stream of various forms of contact with others, which fundamentally modifies their feelings, aspirations and actions. As distinct from other species living in organized communities - such as ants or bees, which are social "by nature" - the socializing processes of human beings establish within them a second nature, which breaks away from the biological order rather than constitutes forms of its expression and continuation. Their relation to the "first nature" - the biological one - is usually believed to be riddled with contradictions. ${ }^{37}$ Homo duplex seems doomed to suffer from this internal and everlasting conflict between impulses originating in two sources of motivation.

which dominated in sociology at the time. However, the departure from this concept was also influenced by increased interest in individual agency, and by the impetus of social changes, which questioned the permanence of social norms (cf. Sikorska 2009). Research whose results could be related to socialization processes is currently conducted primarily by developmental and evolutionary psychologists, neurologists, and neurobiologists. Their work, however, has never been incorporated into the mainstream of sociology and social psychology.

37 This manifests most clearly in Freud's theory, in which culture imposes limitations on primeval human drives, at the same time utilizing their energy to realize its own values through "sublimation." 
This means that contacts underlying human sociality must be essentially different from those observable among other social species. They have to transcend the direct, primary nature of humankind. This conviction served as the point of departure for George Herbert Mead, whose "social behaviorism" is based on the assumption that human interactions are specific insofar as they have a symbolic character: individuals react not to directly accessible actions of those with whom they interact, but to the meanings of those actions ("intentions"). It is the use of symbols, Mead argues, that enables the emergence of intentionality ${ }^{38}$ - the fundamental feature that sets humankind apart from other animals. "In one case the observer sees that the attitude of the dog means attack, but he does not say that it means a conscious determination to attack on the part of the dog. However, if somebody shakes his fist in your face you assume that he has not only a hostile attitude but that he has some idea behind it" $(1972,45){ }^{39}$

However, the most original component of Mead's theory is not his attention to the symbolic character of human actions, but rather the underlying conviction that processes shaping human culture and individual minds are identical. For Mead, anthropogenesis can be in fact seen from two equal perspectives: either as an evolutionary process of the rise of humankind, preceded by the emergence of humanoids, or as a process of shaping individuals who are contemporary to us. In both cases, however, the "humanization of the human" relies on the prior existence of a social environment.

Mead articulates this assumption explicitly in a passage that juxtaposes his views with the prevailing, individualistic account of the mind's nature. Discussing theories that provide us with "the partially (but only partially) social view of mind," Mead indicates that "on this view, though mind can get expression only within or in terms of the environment of an organized social group, yet it is nevertheless in some sense a native endowment - a congenital or hereditary biological attribute - of the individual organism." Thus, "it is not itself essentially a social phenomenon, but rather is biological both in its nature and in its

38 Intentionality is understood here as having an intention (an aim) that the individual is conscious of prior to taking action. Naturally, it is a highly specific interpretation yet one that fits the broadest definition of intentionality as being "directed" onto something. Notably, what emerges here is another element fitting this broad definition: separation of the action's goals (effects) from means (particular forms of behavior allowing to achieve this effect).

39 As Michael Tomasello observes, what makes humans unique is not just the consciousness of one's goal, but primarily "an understanding of them [others] as intentional and mental beings like the self” $(1999,10)$. 
origin, and is social only in its characteristic manifestations or expressions. [...] in direct contrast is our opposite view that mind presupposes, and is a product of, the social process" (1972, 224; emphases added).

Let us pause over the last statement because it has profound implications both for the understanding of the specificity of Mead's thought, and for further discussion of the nature of socializing processes. This statement - often reduced to the thesis that "man is constructed by society," recurring in most works from social sciences - does not seem to be particularly original, especially if adopted as a springboard for an analysis of socialization processes. As Peter L. Berger and Thomas Luckmann (1991) convincingly demonstrated, individuals who enter this world encounter society as objective reality. Thus, they are shaped by this reality with respect to all of their actions, at least because of the properties of their biological equipment. The evolutionary aspect of shaping the human mind has always attracted less attention in sociology ${ }^{40}$ Most interpretations of this thread in Mead's thought create an image of individuals living in a group, who "enter processes of interactions constituted by many individual forms of behavior, directed towards each other and eliciting adaptive reactions" (Ziółkowski 1981, 35). Forming a counterpart to Mead's gestures, these forms of behavior are solidified by way of their repeatability and are consequently invested with social meanings. A particularly important category of those gestures is constituted by vocal ones since exchanging them gave rise to language. As Marek Ziółkowski put it explicitly, in Mead's work "the question of anthropogenesis in fact boils down to the issue of the emergence of human language" $(1981,38)$.

However, many tend to forget that, in Mead's account, this problem cannot be reduced to the rise of language, which is a necessary but not sufficient condition for the development of the human mind. "There has to arise self-consciousness for the whole flowering-out of intelligence. But there has to be some phase of the act which stops short of consummation if that act is to develop intelligently, and language and the hand provide the necessary mechanisms," Mead states. For him, "[s]peech and the hand go along together in the development of the social human being" (1971, 237; emphasis added). He takes the metaphorical

40 In the aforementioned work by Berger and Luckmann this aspect is basically omitted, aside from the enigmatic claim that "externalization as such is an anthropological necessity" (1991, 70). Although these authors' theory gives language a particularly important role in constructing reality (making their approach in many ways a continuation of that developed by Mead), they do not take up the problem of its origins. In their account, individuals are always already equipped with language. This is clearly visible in their analysis of institutionalization (97-109). 
"hand" to be synonymous with actions involving objects since "the human form constitutes its environment in terms of these physical things which are in a real sense the products of our own hands" (249). In this view, the process of "constitution" embraces both the production of objects and their endowment with meanings; moreover, any such meaning is not "intrinsic to the object but arises from how the person is initially prepared to act toward it" (Blumer 1966, 539).

Similarly, the occurrence of the "social process" does not boil down to a purely physical co-presence of other humans, regardless of whether they form a "primordial horde" or one of the many groups existing in today's society. Mead's statement that "the existence of the mind entails the existence of a social process and is its product" ought to be understood literally as a claim that the emergence of properties specific to human intelligence requires a prior existence of some form of sociality. In Mead's view, the human mind acquires its specific features in the process of exteriorizing and sharing the meanings of actions, which cannot be said to have purely biological base (they are not reactions which would be a function of biological conditions), and therefore constitutes an inherently cultural process. Thus, before becoming fully shaped and leaving the state of nature, governed by processes of evolutionary biology, human beings must first produce some symbolic kind of that shared knowledge, and additionally a consciousness of sharing it with others.

Still, adopting Mead's assumption leads to a certain important obstacle. If the mind is formed by breaking away from biology, how could it emerge "naturally"? This problem is usually bypassed by assuming some kind of "growth theory," according to which the first random sounds and the first accidental uses of natural objects have gradually led - through repeatability - to the emergence of language and tools, giving in turn rise to culture, which shapes human mind and facilitates a break away from the process of biological evolution. Such explanation is implicitly contained in a discussion of Mead's ideas by Marek Ziółkowski, who writes that "[t]hanks to mutual reactions to gestures made by two people, there slowly emerged a consciousness of the relationship between one's gestures and another's reactions" (1981, 37; emphasis added).

However, as Jean-Claude Kaufmann notes, such explanations must necessarily be based on the "orthogenesis of the psyche from brain to culture" $(2001,25)$. After all, reactions to gestures made by fellow members of the group also appear among animals but do not lead to the creation of consciousness. Consequently, accepting any variant of the growth theory entails assuming that it is the unique character of the human mind, grounded in biology, which is responsible for the emergence of specifically human cognition. This is precisely the kind of assumption that Mead rejects, mentioning, in the above-quoted passage, "the partially 
social view of mind." As he asserted with confidence, the "mind arises through communication by a conversation of gestures in a social process or context of experience - not communication through mind" $(1972,50)$. Therefore, notions of social process and sociality would become primal in relation to the concept of mind or symbolic culture, which paves the way for a specifically sociological reflection, one that could not be reduced to either psychology or disciplines focusing on culture. The specificity of the human mind would cease to be a point of departure and become a problem to be explained.

Meanwhile, the implicitly or explicitly formulated assumption about the uniqueness of the human mind is still considered obvious not only in social sciences but also in works reporting on latest discoveries in paleontology, anthropology or evolutionary biology. Christopher Stringer and Robin McKie summarize the results of molecular and genetic research on the origins of man, and argue that, in a nutshell, "the biological abyss that once supposed to divide human beings from the animals has been revealed to be the narrowest of genetic crevices. Only a 2 per cent difference separates the genomes (the collective name for an animal's pool of genes) of human beings and chimpanzees, a wafer-thin discrepancy that is nevertheless responsible for all the wonders of our civilization - from plasma physics and Picasso to pizza" (1996, 12; emphasis added). ${ }^{41}$ A similar tone can be detected in Jared Diamond's argument that " $[t]$ he few bits of new baggage we acquired - the 2 per cent of our genes that differ from those of chimps - must have been responsible for all of our seemingly unique properties" (1991, 9; emphasis added).

However, a closer examination of results obtained within disciplines investigating human evolution clearly shows that the conviction about the biological grounds of the specifically human character of mind is based on relatively thin evidence. The problem lies in the fact that the aforementioned genetic difference emerged around six to eight million years ago when the evolutionary lines of chimpanzees and future humans split. Another two million years were necessary for our ancestors to free their hands by assuming an upright position, and further two million to attain the kind of body mass and brain size that approximate the values found among contemporary people. One hundred thousand years

41 According to latest findings, it is even less: ca 1.5 per cent. However, the recently published results of research, which looked not only into genes of chimpanzees and humans but also into the information they carry about proteins, suggest that there is in fact a huge gap between the two genomes, lurking behind the seemingly small difference. We provide this information here for clarification purposes only, since it does not affect our further argumentation. 
ago, the African man was already anatomically identical to today's humans, but further sixty thousand years were required to make what evolution scholars call the "Great Leap Forward," which involved the emergence of the Cro-Magnon man, who led an entirely human kind of life: making tools, building settlements, developing art and starting early forms of religion.

The nature of this "Great Leap" is usually explained by referring to the creation of language, which certainly had biological grounds. The most popular hypothesis links its development with the lowering of larynx and the enlargement of brain. However, this was a process greatly stretched in time: the beginnings of those anatomical changes can be found already in Homo erectus, i.e. around 1.7 million years ago. What is more, some findings suggest that the Neanderthals - now considered an evolutionary branch parallel to that of humanity - had an identical larynx and a 10 per cent larger brain than today's people; still, it was the CroMagnon that prevailed (Stringer \& McKie 1996, 93). The use of tools followed a similar pattern - their first traces are estimated to be 500,000 years old (or even 2.5 million years according to some hypotheses), but they remained very simple and did not change much for hundreds of thousands of years. In fact, "none of those early tools had a sufficiently consistent or distinctive shape to suggest any specific function [...] and the tool names applied by archaeologists may be little more than arbitrary divisions of a continuum of stone forms" (Diamond 1991, 33).

Therefore, it could be said that by focusing on the Great Leap and its origins in brain enlargement, followed by the development of language and tools, researchers of evolution have waved aside the existence of a "Great Hole," i.e. an inexplicably long-lasting period during which biological humans stuck to modes of living that did not set them apart from other primates. This period is simply unaccounted for if we are to think in terms of the above developmental factors. ${ }^{42}$ Similarly, the existence of this "Great Hole" is ignored by social scientists who have adopted various subtypes of the aforementioned growth theory, assuming that typically human cognitive skills were accumulated "slowly" and, at some point, triggered a qualitative leap.

It is the existence of this "Great Hole" that serves as the point of departure for the captivating study written by Georges Guille-Escuret. Already its title, Le décalage humain. Le fait social dans l'evolution ("The Human Shift. Social Fact in Evolution"), signals the desire to seek out the roots of Durkheim's "social

42 There are exceptions to this, though, e.g. Stringer \& McKie (1999) recall the research of Robin Dunbar (1996), discussed below. 
fact." In the first pages, Guille-Escuret expresses his astonishment at the fact that "the mysterious origins of the social fact seem not to attract the attention of specialists" $(1994,7)$. He begins his analysis by arguing that proto-human behavior (primarily the use of tools) can be observed among both primates and early humanoids; however, early forms of pre-human "psycho-culture" differ significantly from what comprises human "psycho-culture" because the former is based solely on the memory of individual representatives of the species, whereas the latter rests upon symbolization. In other words, in the case of pre-human "psycho-culture" the transferring of information accumulated in tools can occur only through learning processes based on imitation. This means that the creation of such "psycho-culture" always has to commence anew in every generation, which significantly impedes the process of development, all the more so because particular skills can be easily forgotten after the death of individuals who were "conserving" them in their actions. ${ }^{43}$

The earliest humanoids still lived entirely within such a pre-human "psychoculture." The transition from Homo habilis to Homo erectus, which occurred in the early Palaeolithic, initially did not affect their behavior despite the fact that from a biological point of view they were already human. Moreover, although "during the process of hominization the volume of the brain increased from 600 to $1,300 \mathrm{~cm}^{3}$ - a change without which humans could not later become passionate inventors who suddenly began to change the fate of the world" - for a long time it was still "the biological sphere that led the ball and set the rhythm" (Guille-Escuret 1994, 275). The "qualitative jump" occurred only in the late Palaeolithic by way of joining words with technological gestures. The beginnings of specifically human features of mind are located by Guille-Escuret in the combined effect of using language and tools. ${ }^{44}$ The use of language sped up the process of transferring information contained in the tool, while language itself

43 The low effectiveness of transmitting "cultural" behavior solely through imitation is also confirmed by results of research made on the habit of washing sweet potatoes, which spread among Japanese macaques, a behavior "invented" by individuals. It shows that the average time of adopting such behavior by a group amounts to over two years (Tomasello 1999, 26-28).

44 This claim is directly supported by results of research on the mental development of children, published in the first decade of the twenty-first century. They show that representations of objects and actions are initially quite alike among infants and other primates. However, unlike other primates, human children creatively integrate these representations around the age of two when they begin to acquire language. Cf. Spelke (2009). 
became another tool, in fact the most powerful one, facilitating the externalization of human memory.

An essential component of Guille-Escuret's theory is the assumption that in the first stage of evolution both processes - i.e. the development of language and the use of tools - took place on two separate levels. Language emerged as a means of coordinating relations among individuals, whereas the transferring of tool-related skills still occurred through imitation. A similar theory was developed by Robin Dunbar (1996), who claims that the primary function of language was not to convey information but to create and uphold relationships as well as determine one's position or status in the group. Dunbar draws attention to the fact that in other groups of primates this function is performed by grooming, which is nevertheless time-consuming and therefore limits the potential number of relationship that an individual can establish, which, in turn, restrains the size of the group. "As group sizes began to drift upwards from the numbers to which apes are currently limited, vocal grooming began increasingly to supplement physical grooming," Dunbar argues $(1996,115)$, simultaneously asserting that the growth of human groups must have been directly influenced by the shift to a nomadic lifestyle. "In effect, a set of neighboring groups would start to act co-operatively $[. .$.$] . The result would be an alliance of loosely federated groups,$ that could come and go, merge and split up, as the mood dictated" (119). In other words, "language evolved to facilitate the bonding of larger groups" (120)..$^{45}$ This contributed to the increase in the number of relationships between individuals and, in consequence, extended the scope of individual experiences possible to share within a group.

45 This hypothesis finds confirmation in two separate sets of data discussed by Dunbar. Firstly, he demonstrates the correlation between brain volume in primates and the size of their groups. For example, gibbons, who have relatively small brains, live in family groups of 4-6, while chimpanzees, who have bigger brains, can assemble groups as big as 50-80. The size of human "herds," estimated on the basis of these relations, ought to amount to ca. 148, which is confirmed both by the size of groups of currently existing hunters-gatherers, and by the size of Neolithic settlements discovered for example in Mesopotamia. Secondly, Dunbar presents results of his analyzes of conversations held at various universities, which show that academic matters took only 14 per cent of the total talking time, while 70 per cent was devoted to personal relations and experiences, constituting the functional equivalent of "grooming." It is also worthwhile to recall in this context another hypothesis, which forms a crucial part of Eric Berne's transactional analysis (1987), namely that of "strokes exchange" as the basis of contacts among humans. 
One needs to keep in mind that language is a system of meanings developed situationally, i.e. in practical contexts of specific actions. ${ }^{46}$ Therefore - as GuilleEscuret argues - as long as the realms of technology and interpersonal relations were not combined, the evolution of human "psycho-culture" was painfully slow. It was only the merger of the two that infinitely multiplied the possibilities for exteriorizing and objectivizing both the technical memory stored in tools, and the social memory preserved in the germs of language, becoming in turn a fundamental factor in the development of both: "language emerged from the sphere of biology through technology and vice versa [...]. Since it became a habit for human beings to somehow simultaneously do what they are talking about, and somehow talk about what they are doing, i.e. since around the late Palaeolithic, they could no longer act without talking about it at the same time (341; emphasis preserved). It is this mutually reinforcing and empowering relationship between the two processes that constitutes, according to Guille-Escuret, the fundamental factor explaining the emergence of both specifically human culture and Durkheim's social fact.

This leads to that aspect of Guille-Escuret's theory which seems to be particularly interesting from the perspective of sociology. According to him, the sudden feedback between language and tool (or between speech and hand, as Mead would have it) led during the first stages to the deregulation of actions so far controlled by biologically conditioned habits. Therefore, the biological basis for the coordination of actions undertaken in the world had to be replaced with a different yet equally comprehensive basis of symbolic and intentional character; the drive-based orientation towards material phenomena had to be supplanted by a reflective orientation towards "intentions" and "causal forces" hidden behind them, while the community of needs - including the "community of fear" - had to be replaced with a community of knowledge supplemented with a consciousness of belonging.

To put it differently: as long as the discharging of still basically animalistic drives could not easily fit in sufficiently coherent, legitimized and stable patterns and institutions, the emerging world of human "psycho-culture" functioned as a destabilizing factor (cf. also Gehlen 1993). It had to be integrated in the act of "foundational holism" (in Jean-Claude Kaufmann's phrasing), which would facilitate directing and limiting the possibilities of human choices, stored in individual memory, and would simultaneously lend them meanings that go beyond

46 Already Lev Vygotski (1994) underscored the importance of this fact for the process of individual language acquisition. We shall return to this later. 
the situational "here and now." The construction of a meaningful order occurred not simply through classification of surrounding reality, but primarily through the creation of a holistic, symbolic image of the world and its legitimization by reference to the sacred. ${ }^{47}$

The fact that this image took a religious form has minor significance in this context. It is much more important to answer the question about the factors responsible for its development. After all, in light of Guille-Escuret's hypothesis, the mechanisms that contributed to the creation of a symbolic view of reality also have to be responsible for socializing processes. Surprisingly enough, the pursuit of this question can greatly benefit from conclusions draw in research into Palaeolithic cave art.

Since they were first found, cave paintings have fascinated scholars, provoking them to formulate opposing hypotheses. The contentious issue has been whether these works express aesthetic needs of Palaeolithic artists, a kind of art for art's sake, or belong to the sphere of magical rituals, in which representations of various animals are supposed to secure success in hunting. The former hypothesis might be disproved by the fact that these pictures almost never show humans, and even if they do, they are greatly simplified or even ineptly made, unlike the ones presenting animals, which abound in naturalistic details and are full of artistic expression. At the same time, however, the hypothesis about hunting magic is also questionable because many paintings present animals that prehistorical hunters did not actually track, which makes it difficult to convincingly argue why they would be found on ritualistic images.

Nonetheless, most scholars agree that cave paintings are testimony to their authors' unique abilities to think in symbolic terms and to intentionally communicate specific information. This conviction has been variously expressed. For instance, Neumann argues that "each of these painted animals is the embodiment and essence of the animal species. The individual bison, for example, is a spiritual-psychic symbol; he is in a sense the 'father of the bison, the idea of the bison, the "bison as such" (1971, 86; after Humphrey 1998). Deacon, in turn, claims that " $[\mathrm{t}]$ he first cave paintings [...] are the first irrefutable expressions of a symbolic process that is capable of conveying a rich cultural heritage of images

47 The same idea is found in Arnold Gehlen who - in drawing attention to old myths relating how gods established the order of the world by overcoming chaos (1993) supports Durkheim's thesis that "[b]asically, the concepts of totality, society, and deity are really just different aspects of one and the same notion" $(2001,337)$. It is also worthwhile to recall the original Latin meaning of re-ligio: "to bind what was disconnected." 
and probably stories from generation to generation" (1997, 374; after Humphrey 1998). However, the English psychologist Nicholas Humphrey, a specialist in the evolution of the human mind, argues (1998) that there is evidence of something contrary: the creators of cave art did not reach the stage of conceptual thinking and did not manifest greater interest in communicating anything.

Humphrey draws attention to the similarity between Palaeolithic cave images and drawings made by autistic children. The data he refers to comes from a study about a six-year-old autistic girl Nadia (cf. Selfe 1985; quoted after Humphrey 1998)..$^{48}$ She began to manifest a special talent for drawing already at the age of three. Her works shared no features with drawings made by non-autistic peers, but displayed a stunning similarity to the best-known paintings from the Chauvet Cave in terms of content, form, and composition.

Similarities between Nadia's drawings and cave art inspired Humphrey to formulate the hypothesis that the way of thinking typical for prehistoric artists was at least in some aspects - closer to autistic modes of cognition rather than those of today's people. Humphrey draws particular attention to the aforementioned lack of human figures on cave paintings, linking it with the hypothesis made by Guille-Escuret and Dunbar, whom he quotes, namely that human language initially developed as a tool for regulating interpersonal relations. ${ }^{49}$ This would mean that Palaeolithic artists did not have at their disposal words for the represented animals. It is this lack of proper linguistic means that he deems responsible both for the presence of animal images on cave paintings, and for the specific mode of representing them. As Humphrey demonstrates on the example of Nadia's drawings, who did not know names of either animals or people, the latter actually make an appearance and are presented as naturalistically as the former.

This line of argumentation led Humphrey to conclude that the specific features of Palaeolithic art ought to be treated not as an expression of their authors' highly developed skills in symbolic thinking (as the above-mentioned scholars wanted), but as a proof that people from that period thought in "pre-modern"

48 Autism is a developmental disability characterized by weak sound processing skills, poor understanding of words and impaired planning of actions. Children suffering from it barely speak and do not react to attempts at contacting them in social contexts. Their actions are divorced from reality, guided by drives and momentary emotions, which display little connection with outside conditions.

49 Humphrey also recalls his own research on communication among rhesus monkeys (1974). Its results indicate that these animals are interested in differences among members of the group, and that they are able to categorize them, at the same time entirely ignoring differences among individuals from other species. 
terms..$^{50}$ Their intellectual capacities would be in some ways reminiscent of those found among autistic children. Importantly, Humphrey does not claim that Palaeolithic artists were autistic, but that their cognition approximated that of autistic children. ${ }^{51}$

Humphrey's interpretation supports the thesis about intellectual and emotional chaos that would precede the moment of foundational holism, all the more so because a key role in his argumentation is played by the fact that Nadia's special skills disappeared entirely when therapy resulted in her beginning to master language. Similarly, the specific kind of art represented by cave paintings disappeared eleven thousand years ago. "And the new traditions of painting that emerged over five millennia later in Assyria and Egypt were quite different in style, being much more conventionally childish, stereotyped and stiff. (...) Maybe, in the end," Humphrey concludes, "the loss of naturalistic painting was the price that had to be paid for the coming of poetry. Human beings could have Chauvet or the Epic of Gilgamesh but they could not have both" $(1998,176)$.

Nevertheless, Humphrey's hypothesis does not answer the question about what provoked the transition from visual to language-based representations. In his book The Mind in the Cave (2011), David Lewis-Williams, one of the greatest specialists in cave art, offers a possible answer by formulating the thesis that cave paintings were the work of shamans, who would thereby preserve the visions they saw in a state of ecstasy. Although Lewis-Williams limits himself to explicating the origin of paintings themselves, he convincingly presents links between Palaeolithic rituals, the process of developing specifically human features of cognition and the early stages in the emergence of strictly social relations based on symbolic ties. He argues that a key role was played in this process by the

50 In texts by authors researching the Palaeolithic, the term "modern" is usually used in reference to the entire period after the Great Leap. Humphrey emphasizes that ascribing a highly developed ability to think in symbolic terms to people from that time usually rests on interpreting other artefacts in the context of cave art, which is treated as the highest manifestation of cognitive capabilities. Since the earliest paintings of this type are dated back to 32 thousand years, and the Great Leap happened 40 thousand years ago, Humphrey's interpretation would necessitate moving the date of birth of "modern" thinking almost ten thousand years towards the present.

51 This claim acquires special significance in light of latest findings about autism, which is now viewed as an impairment resulting from insufficient coherence of cognitive processes. "Recent theorizing about the nature of the cognitive impairment in autism suggests that autistic individuals display abnormally weak central coherence, the capacity to integrate information in order to make sense of one's environment" (O'Loughlin \& Thagard 2000, 375; cf. also Thagard 2003). 
fact that those participating in the ritual could reach so-called altered states of consciousness (cf. James 2004, Castaneda 1972). ${ }^{52}$ They would be induced by consuming hallucinogens, listening to rhythmical sounds, fasting for long periods, or meditating. All of these "ecstasy techniques" - as termed by Mircea Eliade (1989) - allow one to enter a state of trance, which can help to cross the boundary between real world and the world of visions, or between consciousness and the unconscious. Usage of those techniques would be also confirmed by the appearance of various types of patterns and geometrical figures - e.g. zig-zags, spirals, rhombuses, circles - on cave images showing animals. According to current research, such pulsating patterns are among the first to emerge in visions induced by drugs such as hallucinogenic mushrooms, or in states of trance achieved with other means.

According to Lewis-Williams, it was the great intensification of specific emotional states accompanying shamanistic rituals that lay at the foundation of the development of symbolic thinking and intentionality. This mechanism is related to the fact that experiencing a state of trance by an entire community has two particularly important properties. First, it opens a path to another world, i.e. that of visions going beyond the material reality. ${ }^{53}$ Second, it creates a bond that brings together individuals participating in the ritual, and unifies them with the universe. This stems from the fact that the state of trance disables those parts of the brain that are responsible for orientation and association - parts that support orientation in space and the sense of being separated from the rest of the world. It is also known that such states are accompanied by important physiological changes in the entire organism, causing the emotions experienced in a state of trance to become "inscribed" in the brain's structure and thus granting them greater significance. ${ }^{54}$

52 Such states belong to a broader category defined collectively as autistic states (i.e. ones divorced from reality), which also includes daydreaming and dreams.

53 Let us note that hallucinations constitute the original act of abstraction. Science is based on having visions too, for example in the case of the ability to discern in reality something that does not exist there in the purely material sense (e.g. identifying relations, links, regularities etc.).

54 Lewis-Williams's hypothesis is confirmed by various studies summarized by Jonathan Haidt, a psychologist of morality, in the book The Righteous Mind (2012). Haidt introduces the notion of a "hive mode" switch to denote a specific adaptation of humanity, developed by selection at aroup level and meant to tighten group bonds. This mode can be triggered in individuals in many ways. Haidt recalls, for example, the concept of "muscular bonding," developed by the historian William McNeill, to refer to ties created by joint performance of synchronized movements (2012, 635-636). 
The above-mentioned conceptions support the hypothesis of "social breakaway," put forward by Guille-Escuret, and simultaneously allow formulating certain assumptions about the very essence of the related process of "creating human beings and the society." In fact, the main consequence of these conceptions is to significantly shift accent from strictly cognitive properties of the human species (as the main factor in the development of human culture and society, assumed in "growth theories") to an interdependence of cognitive and emotional mechanisms. It is this interdependence that we deem to be the right point of departure for further analysis of social processes. Let us also recall that due to the conditions in which this "emotional imprinting" has been taking place - leading human beings from the world of biology into that of culture - the process of socializing initiated by it has acquired several fundamental features.

First, the transformation of an individual from a being that satisfies its desires not only by using the germs of language and tools but also through a developed cultural symbolism, into a fully "humanized being" occurs by tying one to the entire community, not just to particular individuals. ${ }^{55} \mathrm{After}$ all, it is this community that constitutes the superior whole with which every individual is unified in ecstatic states and whose well-being is the aim of the ritual.

Second, as a consequence of tying individuals to communities, a specific mutual dependence is established among their members - the kind that makes the actions of all individuals subordinated to a higher goal defined in terms of the common good, regardless whether it would mean banishing evil spirits, success in hunting, or assuring the favor of deceased ancestors.

Third, it facilitates the development of intentionality because the image of reality created during rituals not only determines a specific correspondence between the nature of the world and actions taken in it or with regard to it but also provides meaning to individual actions by placing them within the framework of a sacred order. The world constituted in shamanistic rituals is not only organized, but also marked in moral categories, i.e. divided into good and evil

However, it usually occurs in the presence of others and creates a special feeling, "as if the existence of individual consciousness had disappeared and been replaced by a single unifying group consciousness" (Hsieh 2010; quoted after Haidt 2012, 511). Interestingly enough, the special meaning of rituals (and emotions evoked by them) for the emergence of primitive forms of religion and for supporting existing forms of sociality was already pointed out by Durkheim, whom Haidt often quotes.

55 This concerns especially rites of passage, which incorporate maturing individuals into society. 
powers, protective and malicious spirits, creative and destructive forces, ones that can be appealed to and ones that ought to be avoided, etc.

Therefore, fourth, the subordination of individual actions to the sacred vision of the world consolidates that order by marginalizing other potential actions.

Finally, the fifth and last point is that a sense of collective spirit, created with "emotional imprinting," establishes (already at the level of behavior) not only similarities but also differences, calling into existence not only the idea of "us" but also one of "them."

The comprehensive and orderly character of primordial religious beliefs, which gives meaning to the world (both natural and human) that surrounds the individual, can be regarded - Guille-Escuret argues - as constitutive of human culture and sociality, the latter emerging in this context for the first time. Concepts developed by Humphrey and Lewis-Williams support the hypothesis that the unification and ordering of human memory, exteriorized through the use of language and tools, did not occur "gradually," i.e. through progressive extension of the scope of reality covered by symbolization, but rather "during a 'cultural Big Bang' when the ordering of everyday reality reached ever higher levels until it became defined what powers are responsible for the functioning of the world and explicating it, and thereby the keystone of the entire symbolic system is established" (Kaufmann 2004, 42), which becomes the guardian of coercion to act in compliance with the developed image of the world. It is this coercion that constitutes the foundation of Durkheim's social fact.

Guille-Escuret's discussion fills a void left in Mead's theory, a gap usually eliminated by assuming some variant of growth theory. ${ }^{56}$ Both theories remain in astonishing accord with each other, at least in terms of their fundamentals. In both cases, it is assumed that the development of language and tools, as well as the change in brain's volume, were a necessary yet insufficient condition of humanizing humanoids. Both accounts emphasize that the process of sociogenesis is prior to that of anthropogenesis. Therefore, "foundational holism" - which Guille-Escuret views as the basis of the Great Leap - would not have a biological character but a strictly cultural one. Similarly, Mead assumes

56 It seems that Mead himself was aware of this void because he attempted to derive the emergence of human language from imitative processes, nevertheless adding so many caveats to this explanation that it is impossible to ascribe this hypothesis to him. Rather, it is more accurate to say that, without having today's knowledge on the matter, he assumed that the development of human language is somewhat given, and took it as the basis for arguing further about the role played by symbolic communication in the development of mind. 
the prior existence of a social process, which acts as the condition of the existence of individual minds.

It seems, however, that the significance of the "foundational holism" hypothesis goes far beyond merely supplementing certain elements of Mead's theory, although this may not be immediately clear. After all, at first glance this theory does not implicate anything that would question what is accepted in sociology as the default view of society, socialization, or even the beginnings of anthropogenesis. Since the publishing of the excellent study by Berger and Luckmann, titled The Social Construction of Reality, in the mid-1960s, the claim that reality in which people function is socially constructed does not meet with much resistance; one could even say that it has become a cliché. It might seem that finding the exact moment when the "foundational construct" was born in the late Palaeolithic is a rather irrelevant addition to one of those assumptions that currently shape the basis of sociological thinking, and on this occasion, a way of doing justice to one classic thinker who displayed admirable perspicacity in locating the roots of social life in religion (cf. Durkheim 2001).

However, at least the last argument ought to invite a close consideration of the consequences of adopting the "foundational holism" hypothesis. As we have demonstrated in the first chapter, contemporary sociology has greatly departed from Durkheim's vision of the social world. What is more, although constructivism became part of the mainstream in social sciences and its key claim began to be quoted on the first pages of handbooks, it has only slightly influenced the fundamental tenets of sociological thinking. ${ }^{57}$ It might even be said that the embracing of constructivist assumptions reinforced sociology's internal split between the micro and macro worlds, individuals and society as a system, or the "experienced world" and the world of objective social structures. ${ }^{58}$

In fact, this is also related to the dominant ways of understanding Mead's theory. Although it could become a theory that enables grasping the nature of relations between the functioning of individuals and the features of community, it has lost some of its crucial properties in works produced by Mead's students

57 Its influence manifests itself primarily in supplementing the list of sociological publications with a number of works devoted to the reconstruction, deconstruction or sometimes even "construction" (using elements that do not create a coherent whole) of images of various portions of reality, occurring in various social collectives.

58 This division is clearly reflected by the very structure of the book by Berger and Luckmann, because its two main parts are titled "Society as objective reality" and "Society as subjective reality." 
and followers, splitting into two separate currents. ${ }^{59}$ Thus, the most original element of Mead's theory was obscured, namely the relationship between the process of forming individual minds and the shape of the environment, both social and natural, which people inhabit and transform through social interactions. ${ }^{60}$ This relationship holds key significance for understanding the essence of socializing processes. However, in order to grasp it fully we have to scrutinize the mechanisms that mold the human mind.

\subsection{Social foundations of the human mind}

At this point, doubts may arise regarding the extent to which it is justified to return to the Palaeolithic (even late) in considerations guided by the question of what is essential about contemporary social transformations. What new light could be shed on them by even the most striking hypothesis on the beginnings of anthropo- and sociogenesis if the present focus is on current phenomena arising from long-lasting process of the development of human societies, whose actors are individuals socialized since at least thirty thousand years?

The answer is simple and may in fact change our perception of both the process of development and actors engaged in it. As Jean-Claude Kaufmann has rightly observed, localizing the historical moment when the "social breakaway" occurred - i.e. the moment of transition from an existence conditioned by biological mechanisms to one determined by culture - is not only a way of providing a missing element that complements our knowledge and helps with analyzes that embrace continuity of human development from Homo habilis to contemporary humanity. It also entails a radical shift in basic assumptions informing analyzes

59 One of them focuses on the process of the emergence of individual self, and is treated in sociology as a theory describing socialization processes. The other refers to social actions, giving rise - in Blumer's interpretation - to symbolic interactionism, which entirely abandons considerations of the concept of society, focusing on individual actions instead. Although it is possible to point out yet another current - that of an interactionist theory of emotions (cf. Turner 2002) - the first attempts to develop this concept appeared only towards the end of the 1970s. So far, it has had little impact on sociological thinking.

60 Let us recall here that along with interactionism and most other contemporary sociological theories that incorporate its findings, there disappeared the concept of sociological group, which plays a key role in Mead's theory. For example, in the structuration theory of Anthony Giddens (1986), which in its fundamental aspects constitutes a specific synthesis of crucial currents present in contemporary sociology, the concept of the group does not appear even once. 
undertaken in social sciences. Among these assumptions, an important place is assigned to the very idea of social development and the vision of reflective individuals, who initiated that development due to their unique genetic equipment.

The connection between social development and individual Reason was established during the Enlightenment, and was consolidated - as Kaufmann notes - by the iconographical impact of the widespread image of the "March of Progress,"61 additionally supported by "our epoch's ideology, which focuses on the concept of sovereign individual" $(2004,24)$. The significance of representations popularizing the process of evolution was also pointed out by Guille-Escuret, who mentions spontaneous mental images appearing in the minds of both experts and amateurs, all equally influenced by such iconography: "We are able to 'see' the growth of brain and the development of the psyche as a march forward, as in the comic strip presenting the gradual straightening of the chimpanzee until reaching a fully erect position of the human being" (1994, 245-246). However, this way of "seeing" is entirely false because in the light of available data it rather ought to be assumed that the development of the human brain - the "mental organ" as Noam Chomsky has termed it - concluded around the late Palaeolithic. We have actually stopped marching then, and have not changed in the biological sense. Brains of contemporary people are exactly like brains of those who lived as hunter-gatherers before and after the "cultural Big Bang;" therefore, it is not any configuration of their "material" properties that should be considered the source of social development.

Before attempting to answer the question regarding this source, let us dwell for a moment on the first part of the above statement. The premise contained in it, which serves as the point of departure for evolutionary psychology, has led some researchers to formulate conclusions that we would definitely disagree with. For example, in his otherwise excellent book on the workings of the human mind, Steven Pinker notes that "[f] or ninety-nine per cent of human existence, people lived as foragers in small nomadic bands"; from this he draws a completely unwarranted conclusion that "[o]ur brains are adapted to that long-vanished way of life, not to brand-new agricultural and industrial civilizations" $(1998,42)$, thus equating the form of the brain with the functioning of the mind. We would

61 Reference is made here to the frequently reproduced yet misleadingly false image supposedly illustrating the basic premise of evolution. It shows a line of humanoids, one more erect than the other, marching under the leadership of contemporary man. Kaufmann borrowed the term "March of Progress" from Stephen J. Gould (1989). 
rather lean towards concepts originating in evolutionary psychology, which seek to explain the ways in which the minds of contemporary people function within a framework of interdependence between them and biological or environmental factors. Among such theories there is the one developed by Daniel C. Dennett.

Like many other scholars, Dennett assumes that the development of intentionality, especially of the second-order kind, which is a specifically human feature involving "beliefs and desires about beliefs and desires, its own or those of others," was "an important advance in kinds of minds" $(1996,121)$. At the same time, he notes that this kind of intentionality also appears among animals. ${ }^{62}$ In that case, though, it assumes the form of "non-reflective proficiency." By observing the behavior of pets, it is easy to conclude that a dog wants us to take specific action (e.g. take it for a walk); thus, it behaves intentionally, i.e. in a way that takes into account its intentions as well as the ones of its owner (e.g. by circling next to the door and gazing expectantly when it sees the owner getting ready to leave). This does not mean, however, that it thinks like us, having access to a representation of the owner's mind, which would facilitate formulation of judgements about our behavior ("he or she wants to go out"). It suffices that a dog has at its disposal "a largish 'list' of alternative behaviors, nicely linked to a largish list of perceptual cues" (124).

According to Dennett, the need for a more direct, easily transformable representation of one's own and other beings' behavior could appear only when those cue-based "lists" began to grow exceedingly complex, which could be only the result of the need to cope with an accordingly complex environment. Let us observe that Dennett's argumentation - resting, by the way, on a strong foundation provided by data from various kinds of research - perfectly fits the (pre)

62 The view of intentionality is that which, on first glance, strongly differs Dennett's concept from another, very popular one (especially among psychologists), developed by John R. Searle (1994). Both outstanding philosophers of mind take as their point of departure the assumption lying at the foundation of evolutionary psychology, namely that human brains are the product of evolutionary processes that concluded in the late Palaeolithic. However, whereas Dennett sees intentionality merely as a certain stage in the development of animate minds, Searle perceives it as something inherent only to humans, in contrast, for example, to artificial intelligence. Differences in the "frame of reference" assumed by the two thinkers, and in the distribution of emphasis Searle focuses on "phenomenological properties" of mental states considered from the perspective of individual emotionality, whereas Dennett assumes the position of an outsider observing species-specific behavior - lead to a certain divergence in the very understanding of the concept of intentionality. This, in turn, results, in the exaggeration of differences between the theories of these two authors. 
historic sequence of anthropogenesis as reconstructed by Guille-Escuret (and described in the section above). The increasing complexity of the environment, which the former assumed, would correspond to the process that preceded the moment of "social breakaway" - the process that, according to Guille-Escuret, consisted in "combining" the germs of language with early tools. Therefore, if as Dennett puts it - "thinking - our kind of thinking - had to wait for talking to emerge, which in turn had to wait for secret keeping to emerge, which in turn had to wait for the right complexification of the behavioral environment" (130), it was in the period of the Great Leap that the volume of new information increased rapidly. This bulk of information was "encoded" in new tools, and was related to the means of their creation and use. This could be the "secret" that accelerated the development of language and consequently contributed to the shaping of early modes of thinking.

According to Dennett, the use of tools was the primary factor in the transformation of people from "Popperian" beings whose "internal environment" was still constructed by perceptual representations - which nevertheless already allowed for reviewing a range of alternatives, equipping those beings with some degree of intentionality (as in the case of animals) - into "Gregorian" beings that use the outside environment as a source of various "devices" that help them in improving their efficiency, raising precision in testing hypotheses, and making decisions, ultimately turning them into truly intentional actors. Among those "devices," the earliest ones were tools. Using a handheld hammerstone not only required intelligence from those individuals who "invented" ways of using naturally formed stones or forming them to better fit in their hands but also increased, in turn, the intelligence of all its potential users because every tool contains accumulated information about the actions it makes possible and relations it can help establish. However, it was language that proved to be the most powerful "device." It immensely boosted the ability to represent the external environment in the "internal" one of individual psyche, as well as increased people's capability of establishing cooperative interdependencies among themselves.

Still, neither the invention of ever more perfect tools nor the development of language has introduced any significant changes to the human brain. As we have already noted, most researchers investigating this phenomenon assume that the formation of the human brain as a specific "mental organ," which began with the increase of its volume, actually concluded in the late Palaeolithic. Therefore - as Dennett writes - although "[o]ur brains are modestly larger than the brains of our nearest relatives [...], this is almost certainly not the source of our greater intelligence" (134). Not even the biggest brain is capable of storing all the potential information preserved in language and tools. According to Dennett then, 
" $[\mathrm{t}]$ he primary source $[\ldots]$ is our habit of off-loading as much as possible of our cognitive tasks into the environment itself $[\ldots]$ into the surrounding world, where a host of peripheral devices we construct can store, process, and re-present our meanings, streamlining, enhancing, and protecting the processes of transformation that are our thinking. This widespread practice of off-loading releases us from the limitations of our animal brains" (134-135; emphasis preserved).

Let us dwell for a moment upon this statement, as it has extremely far-reaching consequences for the understanding of socializing processes, which are the subject of this chapter, and which are assumed - either implicitly or explicitly - to be the foundation of all sociological concepts. After all, if neither intentionality nor intelligence are "natural" properties of the human mind, codified in specific features of the human brain, it means not only that we have to question the vision of man as the culmination of the "March of Progress," and therefore as the "natural" (i.e. having inborn properties) "ruler of all creation," but also - and perhaps primarily - that we need to ask about the mechanisms of shaping those properties, whose existence is not undermined here because they are well documented in various kinds of research. Although the first conclusion is important mainly because of our own feelings, and can be thus left without commentary, the question that arises from it has a fundamental significance for all considerations of how human beings and societies function.

Dennett's answer to this question is relatively simple: "Every human mind [...] is a product not just of natural selection but of cultural redesign of enormous proportions" $(1996,153)$. The basic tool for this transformation is language itself, which we acquire during childhood. It constitutes one of those "peripheral devices" that allow the human mind to acquire its unique properties. However, language is not the only tool used to externalize representations of reality produced by our actions. "We keep 'pointers' and 'indices' in our brains and leave as much of the actual data as we can in the external world, in our address books, libraries, notebooks, computers - and, indeed, in our circle of friends and associates. A human mind is not only not limited to the brain but would be severely disabled if these external tools were removed [...]. Furthermore, [...] the more intimately familiar you become with the peripheral objects thanks to your practice in manipulating them, the more confidently you can then do without them, sucking the problems back into your head and solving them in an imagination disciplined by its external practice" (144-145).

Dennett's answer is seemingly obvious, even banal, because it refers us back to one of the axioms of sociology, namely the belief that human actions are culturally conditioned. However, let us draw attention to the fact that this belief in no way undermines the aforementioned assumption about the special properties of 
the human mind and its "natural" predisposition to acquire and produce knowledge. ${ }^{63}$ The only caveat is that this knowledge may be culturally differentiated. Meanwhile, in Dennett's theory the specifically human intentionality and intelligence are in no way a "natural" property of humankind, but rather appear as derivative of the usage made by individuals of culture understood as exteriorized and accumulated information, something like an enormous social repository of memories, holding all kinds of products of human actions. ${ }^{64}$ This means, in turn, that if we want to answer the question about what contributed to human development, we first need to answer another question - one about the kinds of mechanisms that allow people to make use of culture.

Scholars started to search for answers to this question only in the 1990s, when many publications appeared which shed new light on how the human mind operates. One such study is Descartes' Error by Antonio R. Damasio (1995), which discusses the results of research on the functioning of patients with reduced sensibility due to frontal lobe damage from an accident or surgical intervention. These findings unequivocally confirm that despite not having lost any degree of their social knowledge, those people nevertheless were severely impaired in terms of their ability to make rational decisions. Although in laboratory conditions they were capable of logically analyzing problematic situations presented to them in test exercises, and proved able to arrive at all possible options to act, they were at the same time unable to select from these options one they would consider to be the best course of action. Generally, it could be said that due to the inability to feel emotions, the internal decision-making mechanism was damaged to such an extent that the patients' behavior became "silly and

63 One typical example of such thinking is Piaget's 1920s theory of cognitive development (cf. e.g. 1979), in which the movement through individual stages, characterized by the growing complexity of the mind's intellectual functions, occurs as if automatically and without any relation to cultural differentiation. Such a conceptualization implicitly assumes the intervention of mechanisms that have a biological basis. This theory was questioned from two positions. First, many experiments have confirmed that Piaget did not appreciate enough the actual cognitive skills of children (cf. e.g. Pinker 1998, 302-342); second, that he did not recognize the influence of the social environment on cognitive development (cf. e.g. Vygotsky 1994; Greenfield \& Bruner 1966; Scribner 1979; Tomasello 1999). Social sciences' attachment to the vision of mankind as the "crowning of evolution" is most distinctly confirmed by the fact that despite criticism and Piaget's later attempts to soften his "biological universalism" (1979), his theory is still quoted in contemporary psychology handbooks in its classical form.

64 The consequences of such an understanding of culture is discussed in the next chapter. 
irrational," especially in situations involving personal and social dimensions. As a result, they have become unable to effectively function in society.

Having analyzed a large number of such cases, Damasio formulates a hypothesis about so-called somatic markers, which - he argues - refer to special kinds of emotions that have been linked, in the process of learning, with foreseeable future effects of certain scenarios describing possible courses of events. His use of the term "somatic" is supposed to emphasize the presence of physiological experiences serving as an "early warning system" that eliminates certain options already at the very onset of the decision-making process, or at least inclines one to perform a more detailed analysis of possible consequences. Thanks to this, somatic markers accelerate decision-making because they relieve us of the duty to analyze all possible ways in which a specific action may be taken. Simultaneously, they allow to hierarchize the pool of all potential decisions.

Some of the markers defined in the above way stem from in-born, primordial emotions, e.g. fear of certain stimuli or their aspects. However, most markers have been formed under the influence of our experience: by linking specific categories of stimuli with specific categories of somatic states. Generally, it could be said that "[s]omatic markers are thus acquired by experience, under the control of an internal preference system ${ }^{65}$ and under the influence of an external set of circumstances which include not only entities and events with which the organism must interact, but also social conventions and ethical rules" (1995, 179). The basic group of stimuli brought under this kind of somatic control is instilled in childhood and adolescence, but the process of developing somatic markers in fact lasts throughout human life.

Damasio's hypothesis of somatic markers leads to a complete overhaul of the role played by emotions in human actions, as it was assumed in social science. Regardless of the fact that emotions were marginalized for a long time as a field of inquiry (in academic sociology and social psychology alike) ${ }^{66}$ most theories

65 As Damasio writes, "[t]he neural basis for the internal preference system consists of mostly innate regulatory dispositions, posed to ensure survival of the organism. [...] The internal preference system is inherently biased to avoid pain, seek potential pleasure, and is probably pretuned for achieving these goals in social situations" (179).

66 In a relatively new, 600-page-long handbook (Aronson et al 2015) the subject of emotions is discussed on only several pages. The interactionist theory of emotions developed since the 1980s (cf. Turner 2002) has not won greater recognition so far. It was only in the last years of the twentieth century that this topic began to attract greater attention in social sciences, popularized through the concept of so-called emotional intelligence (cf. Goleman 1995) rather than any earlier theoretical endeavors. 
subordinated them to cognitive processes. In the frequently quoted "doublefactor" theory of emotions (Schachter \& Singer 1962; cf. also Aronson et al 2015), emotions - or, to put it in more precise terms, their mental representations, or impressions of emotions - are the effect of intellectual elaboration of the physiological stimuli we experience. A similar perspective can be identified in analyzes that begin from raising the question about the influence of emotions on human behavior - in this case researchers have also focused primarily on how emotions distort perception. In both cases, it is not the emotion but the cognitive schemaat best modified by emotions - that is responsible for the final shape of individual behavior. The results of such research seem to suggest that human beings could just as well do without emotions, or at least that emotions disturb us rather than help in adapting to the surrounding world. In the best case, such results reinforce the popular conviction that emotions make human behavior irrational. Meanwhile, in Damasio's hypothesis of somatic markers emotions not only cease to be viewed as factors disturbing rational behavior, but become a sine qua non condition for the process of taking "intelligent" decisions.

Stanley I. Greenspan goes even further in considering the relationship between emotions and individual cognition to describe the former as the fundamental mechanism of shaping the "fully human mind." For Greenspan, the significance of Damasio's markers hypothesis lies primarily in renewing interest in the role played by emotions in the realization of complex intellectual tasks. ${ }^{67}$ At the same time, however, he emphasizes its inherent flaw by indicating that this hypothesis preserves "the historical dichotomy between cognition and affect [...] showing that brain injuries can affect emotions and therefore judgement while leaving critical aspects of cognition unaffected" (Greenspan \& Benderly 1997, 7). In his view, preserving this dichotomy does not allow to discern the real role played by emotions in shaping the most fundamental aspects of the human mind. ${ }^{68}$

According to Greenspan, this role boils down to two fundamental ways in which emotions participate in socialization processes. The first is related to the

67 We use the word "renewal" because this topic was already explored at the beginning of the twentieth century by Sigmund Freud, but it was later marginalized in social sciences due to the dominance of research conducted from a cognitive perspective.

68 Let us point out here that the difference between the two scholars can be rooted in the methodological differences that underlie the conclusions drawn by them about the mind's functioning. Damasio studied adults in whom there occurred an actual dissociation of emotions from cognition due to mechanical injuries to those parts of the brain that control emotions, whereas Greenspan studied autistic children whose brains - in the physical sense - were not damaged but did not create certain connections. 
shaping of a specifically human intentionality that entails the ability to distinguish emotional signals, to read the others' intentions and communicate one's own, which, in turn, enables one to take deliberate action. Acquired relatively early in the process of socialization, "the intuitive ability to figure out human exchanges, to pick up affective cues before any words have been exchanged and understand their significance, eventually comes to function as a kind of sense organ. Indeed, it becomes a sort of 'supersense' that subsumes elements of all the others and lets us make instantaneous assessments and adjustments in our own reactions. It is in fact what makes social life possible" (64-65; emphasis added).

Initially, however, this "supersense" functions on a purely behavioral level, both in terms of emotions felt by children and those experienced by adults. This is the level of mental representations that, together with mastery of language, would be perfectly sufficient to acquire purely "technical" cultural skills in the form of behavioral patterns necessary to satisfy the needs of individuals and enabling them to direct the behavior of others. However, stopping at this level of development would prevent, according to Greenspan, the full development of mind. "Optimal mental development - what we have called mental health requires a feeling of connectedness with humanity, a well-developed sense of empathy, the ability to express and evaluate abstract concepts (including values such as justice, fairness, etc.), the individual's sense of her place in relation to the larger community, an understanding of consequences, a capacity to weight alternative values and to place her own wishes in the context of other's wishes and needs, and an ability to recognize legitimate authority and limits" (193-194). Here, emotions re-enter the stage, because to master those kinds of skill it is absolutely crucial for the child to have "a warm, close relationship with an adult, one in which communication becomes important enough to provide satisfaction in itself" (77). Without reliable emotional responses from an adult, who helps the child in translating emotions and behavioral stimuli into the language of feelings and motives, those skills might never develop.

Greenspan's findings are complemented in a way by those of Michael Tomasello, who demonstrates, using a series of cleverly designed experiments, that although the cognitive skills of all primates are very similar, it is human cognition that is distinguished by the ability to attain a shared intentionality, understanding others "as intentional and mental beings like the self" $(1999,10)$. Neither other hominids nor autistic children have similar motivations to share emotional states and establish strictly psychological relationships among themselves, as is the case with most children.

The existence of this "we-intentionality" manifests itself at a relatively early stage of the development of human beings ("the nine-month revolution"), 
immeasurably increasing the effectiveness of absorbing cultural heritage, and readiness to engage in joint actions. According to Tomasello, this "uniquely human cognitive adaptation for understanding others as intentional beings like the self" (40) triggers processes of cultural learning and internalization, at the same time confirming that "human beings 'identify' with con-specifics more deeply than do other primates" (14). Moreover, he is convinced that the specificity of human learning processes, which is supported by emotions, fostered cumulative cultural evolution. "We may conclude, then, that whereas chimpanzees clearly create and maintain cultural traditions broadly defined, these very likely rest on different processes of social cognition and social learning than the cultural traditions of human beings. [...] a crucial difference emerges, and this manifests itself in processes of cultural evolution, that is, processes by which a cultural tradition accumulates modifications over time" (37).

Given all the ways in which the concepts discussed above differ, owing primarily to diverse interests of individual authors, they do form a coherent message. The results of research conducted by Greenspan and Tomasello clearly indicate that emotional ties established between the child and its social environment lie at the foundation of the development of the individual mind. Regardless of whether we call it "shared intentionality" or "emotional supersense," it turns out that the "cultural transformation of the mind" assumed by Dennett takes place by way of incorporating the individual, from the moment of birth, into a complex network of emotional interactions with adult representatives of the same species. Furthermore, it is important that this transformation is inseparable not only from the transmission of various technical skills specific to a given culture - the use of language, tools, and at least some elements of knowledge but also, perhaps even primarily, from the incessant emotional qualification of actions taken by the child. It is highly probable that this process serves as the foundation for the forming of at least some somatic markers, whose existence is assumed by Damasio.

At the same time, however, these concepts leave us unsatisfied, despite being formulated in the language of universal dependencies. On the one hand, they are strongly tied to normative components of contemporary culture. ${ }^{69}$ On the other

69 This is related in particular to Greenspan's theory. After all, it is enough to recall what is already known about the treatment of children in various historical periods and in different cultures (Aries 1965) in order to raise doubts about the universality of Greenspan's claim about the necessity to express "ecstatic admiration" of the infant as a condition of his or her optimal mental development. 
hand, they utterly disregard processes of its differentiation, which occur both diachronically and synchronically. By bringing to the fore the adaptive aspect of socialization processes,${ }^{70}$ they leave aside the question of historical variability of human culture. In other words, even though they answer the question about the mechanisms that allow people to make use of culture, they nevertheless fail to address the one regarding the way in which culture is produced by human communities and societies.

Of course, this is not a charge that could be levelled against these particular theories. Still, the very existence of an interdependency between the shaping of individual mind and the requirements of culture - or more broadly speaking, of society - compels us to closely investigate the relationship between two kinds of socializing processes.

\subsection{Two dimensions of socializing processes}

As Piotr Sztompka notes, "the problem of socialization emerges when we wonder where people obtain knowledge, convictions, skills, rules, norms and values they later use in their actions. In order to act, we all need to have some data, information, behavioral patterns, ideals, models and visions of goals, which guide our actions. Where do they actually come from?" $(2002,390)$. This question has been variously answered in diverse theories and in different epochs, which does not change the fact that analyzes of individuals' socializing processes, undertaken in social sciences and indicated by the concept of socialization, usually boil down to analyzing mechanisms responsible for introducing culturally differentiated

70 This, in turn, is particularly visible in Damasio's theory. Analyzing the relationship between somatic markers created in the process of socialization and the shape of culture, he claims that "[t]he buildup of adaptive somatic markers requires that both brain and culture be normal" (177; emphasis added). As an example of the brain's "abnormality," he quotes developmental psychopaths and sociopaths, who are "yet another example of a pathological state in which a decline in rationality is accompanied by diminution or absence of feeling" (178). On the other hand, cases like Hitler's Germany, Stalin's USSR, China's Cultural Revolution, or Pol Pot's Cambodia are in his view an illustration of the situation in which "a sick culture prevailed upon a presumably normal machinery of reason" (179; emphasis added). Sharing Damasio's negative opinion of these particular systems, let us indicate, however, that a naïve belief in the existence of a "normal" culture and a "normal" mind leads to a contradiction within his account: if somatic markers perform an adaptive function in relation to culture, then the latter does not have to "prevail" over anything, and even if it did, it would mean that the sources of these markers are outside culture. 
contents into their minds, but entirely pass over the question of the essence of sociality, which is a parallel process. In other words, just like the question "What is society?" has been replaced in sociology with descriptions of how societies function, the question about the real nature of socialized individuals has been obliterated and supplanted by the claim that there is no such thing as a nonsocialized individual (vide the cases of "wild children").

Nothing is strange in this because each of these questions concerns a different aspect of the same phenomenon - that of social life. This was particularly emphasized by Durkheim, who wrote that "[o]n the one hand, the individual takes from society the best of himself, everything that gives him a distinctive personality and a place among other beings, his intellectual and moral culture. Take away language, the sciences, the arts, and moral beliefs, and he falls to the level of brutishness. The characteristic attributes of human nature therefore come to us from society. But on the other hand, society exists and lives only in and through individuals. Extinguish the idea of society in individual minds, let the beliefs, traditions, and aspirations of the collectivity cease to be felt and shared by the particular people involved, and society will die" (2001, 257).

However, in order for the idea of society to be upheld by individuals, it had to be formed first. This is the proper significance of the hypothesis of "foundational holism," for its logical consequence is the statement that the creation ${ }^{71}$ not only of the social fact but also of human beings and society took place in the same process - a singular "cultural Big Bang" occurring in a condensed period, whose effect was the production of a total, symbolic image of the world. Naturally, before this event people already had their specific genetic features (the symbolic two per cent separating humans from other primates), which allowed them to make the transition during the Great Leap: from an existence conditioned by biological mechanisms to one determined by social mechanisms. Nevertheless, since the end of human evolution these features did not significantly impact their behavior for thousands of years. Similarly, one could argue that even though human beings lived in family-centered groups since the beginnings of their biological emergence, this did not turn these groups into societies. Nor does this happen among other social animals, which we call by this name only metaphorically.

71 Naturally, we are not leaning towards some version of creationism here, but wish to emphasize the movement from those forms of pre-human psycho-culture that precede the Great Leap to forms characteristic for contemporary people - forms whose emergence dates back precisely to the time of the Great Leap. 
Therefore, the process of "humanizing the human being" and "socializing human societies" cannot be deduced either from unique features of the human mind, or from the existence of any "social instinct" so eagerly invoked by nineteenth-century thinkers. There had to be something else - an additional factor that could "trigger" the potential inherent in the biological equipment of human beings. As discussed above, according to Guille-Escuret it was the moment when people began to combine the use of language with the use of tools $\mathrm{s}^{72}$ which enforced the unification of various elements of human proto-culture to form a total image of the world. The circumstances accompanying this process triggered the processes of anthropo- and sociogenesis, for it was the sharing of meanings contained in the image of the world that gave the human mind its specific features, facilitating mutual reading of intentions and representing them as Mead stressed - before proceeding to act. It not only led human beings out of the world where everything was conditioned strictly by biology, but at the same time offered a basis for the emergence of a collective "We" - a counterpart to Mead's "general Other."

Localizing the moment of "foundational holism" after the evolution of the human species had ended entails serious consequences for the understanding of socializing processes, both in their collective and individual dimension. The culture produced in the act of foundational holism and later modified in the course of historical transformations, is not just a random assembly of artefacts, symbols and ideas. "Culture," as Max Weber emphatically put it, "is a finite segment of the meaningless infinity of the world process, a segment on which human beings confer meaning and significance" (Weber 1949, 81; emphasis preserved). Therefore, the emotional marking of potential actions, which occurs

72 Another immensely fascinating hypothesis was developed by Lewis-Williams (2011), who has indicated the significance of the encounter with Neanderthal groups for humans of the Aurignacian culture during their movement to Western Europe. His argument is largely devoted to proving that the Neanderthals had a different kind of consciousness, which was limited to such cognitive abilities that Gerald M. Edelman (1992) termed "primary consciousness" and which roughly correspond to Dennett's lower-order intentionality characteristic also for certain species of animals. LewisWilliams is convinced that, having noticed these limitations, the Cro-Magnon felt "a sense of superiority over the Neanderthals [...] [which] coloured their relationship with them" (2011, 261-262); consequently, this could have contributed to the heightened intensity in exploring their own cognitive capabilities. Still, Lewis-Williams's hypothesis does not undermine that of Guille-Escuret, but rather interestingly supplements it. 
in the process of collective and individual socialization, and guides the "uses" that individuals can make of cultural resources, does not define their personal preferences but establishes normal functioning in the world, which they view as something obvious. ${ }^{73} \mathrm{~A}$ similar situation is found in the case of "shared intentionality," which serves as a basis for the development of a sense of collective "We." Understanding others as "intentional and mental beings like the self" (Tomasello $1999,10)$ makes it possible to bind individuals emotionally to the larger collective; however, it also carries the potential to exclude those whose aims, intentions and actions do not fit the pattern we have been trained to endorse in the process of socialization.

It needs to be emphasized that in this account the crucial stages in the process of socialization occur outside conscious control of either the socializing or the socialized. "We learn about empathy and compassion not from what we are told but from how we are treated," Greenspan notes $(1997,120) \cdot{ }^{74}$ He points out that the roots of morality are located in the pre-verbal stages, which are nevertheless deeply saturated with emotions. They are full of interactions with adults, in the course of which a child becomes aware "of her own and other's intentions, which take in such basic issues as safety versus danger, acceptance versus rejection, approval versus disapproval, pride and respect versus humiliation" (120). When reacting to children's behavior, "adults do not devise these gestural interactions randomly. Rather, they base their behavior on their own values and sense of rightness and correctness - on what the smiles, scowls, hugs, shrugs, nods, and angrily turned backs taught in their own experience" (290).

Therefore, "gestural communication imparts basic information about how a society functions" (290). However, if such information remains devoid of the emotional ties established between individuals and their social environment, it can only lead to the development of a technical skill allowing for more or less

73 Weber's "reference to value" means precisely a system of preferences, which guides individual action. What is worth emphasizing, Weber differentiates in this context between individual values ("egoistic" and oriented at one's own well-being) and social values (oriented at the good of the community). According to him, the former are typical for the modern order, in which behavior is marked by a high degree of reflectivity. On the other hand, social values accompanied by low reflectivity, i.e. affective ones, are typical for the traditional order. Just like Simmel, Weber argues that in the process of social change there is a visible trend to subdue emotions as a basis for action.

74 Let us note that this is a kind of a return to an idea developed in the 1950s within the so-called theory of social learning (modelling) associated with two scholars: Albert Bandura and Richard H. Walters $(1959 ; 1967)$. 
effective manipulation of the surroundings in order to satisfy one's own needs. The same view was expressed in slightly different terms by Marcel Gauchet, who notes that socialization involves not only "the incorporation of the habits and rules that make collective coexistence possible" but also "the process through which one learns to perceive oneself as somebody among others" (2000, 31; emphasis preserved). In the second meaning, socialization would be about "learning to abstract from oneself that sensitizes us to the public, to objectivity and to universality; it allows us to adopt the point of view of the collective and abstract from our particular situation" (31; emphasis added).

Both scholars also claim that the changes occurring in contemporary society significantly limit the chance to develop this kind of emotional ties. For Greenspan, the key characteristic of these changes lies in the increasing impersonality of social life, in which contacts with close people are supplanted by contacts with strangers, while contacts with people in general - by contacts with technological devices. This significantly limits the possibility of individuals entering into the kind of emotional interactions that serve as the condition for realizing the "optimum mental development," which links the more technical skills with those mental properties that allow the individual to unite with a broader community. Gauchet, in turn, assesses that the roots of this deficiency can be located in processes of individualization, which he views as a complex effect of changes in the forms of organizing society. In other words, whereas Greenspan's theory supports the popular thesis about the breakdown of ties in contemporary society, Gauchet's arguments could be directly deduced from Beck's discussion of the process of structural individualization.

Both scholars actually add something to these findings because their discussion of changes in socialization involves a specific differentiation between elements that are part of this concept. This allows to distinguish two basic aspects of it. In the weaker sense, it would be synonymous with "the process thanks to which the individual becomes used to a new way of life in his group and broader society through learning the rules and ideas contained in culture" (Sztompka 2002, 391). This understanding of socialization dominates in contemporary social sciences and academic textbooks. ${ }^{75}$ As Gauchet rightly observes, "[i]n this minimal sense - as adaptation to life with others - the socializing capacity of contemporary families is neither better nor worse than it used to be" $(2000,31)$. However, both Gauchet and Greenspan link processes of socialization with its second,

75 It seems that also Dennett, in his theory of the mind, focuses primarily on the "efficiency-related" aspect of socialization. 
much stronger understanding: socialization as a mechanism not only responsible for the introduction of culturally differentiated contents into individual minds, and the development of methods in which these contents are processed, but also the condition for the development of such attitudes that would facilitate reproducing and sustaining social life by way of tying oneself emotionally to the collective "We." Without effectively realizing the latter function, socialization may impair the processes of socializing the actions of individuals.

For both authors, it is this deficit in socializing individuals' actions that endangers the very foundation of contemporary society. Gauchet associates this threat with such transformations of personality that radically complicate, and perhaps even preclude, the realization of the idea of civic duty. In his view, the fundamental property of the contemporary individual is that he "would thus be the first individual to live unaware that he lives in society, the first individual, due to the very evolution of society, able to ignore that he is in society" (2000, 36). For Greenspan, in turn, this threat would be closely linked to the fact that specific properties of the human mind - among which he lists primarily intentionality and the ability to empathize - have their roots in complex, emotional interactions. According to him, contemporary society undermines the very foundation of the human mind by reinforcing impersonal aspects of social life, which may lead to the destruction of the main basis for its achievements.

Perhaps, the proclamation of the end of society is too hasty in both cases. If we are to follow the stronger definition of socialization, understanding it as a process of incorporating the individual into a network of relations that constitute a given society, then the decreasing efficiency of that process - emphasized by Greenspan, Gauchet and many others - can simply mean that we are entering a period in which these relationships are radically changing their character. It would not be anything strange because one such transformation already occurred in the history of human societies. ${ }^{76}$ We should rather investigate more closely what creates these relationships and how they correspond to the currently unfolding changes in the forms of socializing processes.

76 What we mean here is, naturally, the transition from the traditional society to the modern one, analyzed by classical scholars in terms of such oppositions as Gemeinschaft versus Gesellschaft (Tönnies 2001) or mechanical solidarity versus organic one (Durkheim 2013). 


\title{
CHAPTER THREE. TRANSFORMATIONS OF SOCIAL BONDS
}

\author{
Every culture defines itself in relation to nature; every \\ culture makes use of a grid to integrate and encode gods, \\ humans, animals, and things.
}

PIERRE VIDAL-NAQUET, The Black Hunter

Human imagination had been shaped to suit tribes that were small enough to move quickly, yet strong enough to defend themselves.

RYSZARD KAPUŚCIŃSKI, Living a Double Life

The concept of social bond belongs to those elementary sociological notions whose meaning has undergone banalization and simplification, actually precluding any precise reconstruction of its sense. Although it is now commonly used and increasingly often plays a crucial role in accounts of contemporary society, which is said to be haunted by the "erosion" or "dissolution" of these bonds, analyzes of contexts in which it is employed show, at best, that this term is usually given two fundamental meanings - or more precisely, two categories of meanings. In the first case, the idea is used to denote all kinds of links formed between people, thus making it the opposite of individualization. ${ }^{77}$ In

77 This is how Ulrich Beck seems to use the concept of social bond. In the sociology handbook by Robert Nisbet and Robert G. Perrin - notably titled The Social Bond - the only reference to the eponymous term is contained in the claim that the basic problems of sociology are linked to the nature of social bonds, i.e. "the mechanisms and processes through which human beings become members of the social order and by which they remain members" (1977, 37-38). In Polish sociology, a similar approach is represented by Jan Szczepański, who considers social bonds to be "fundamental concepts in sociology," defining them as "an organized system of relationships, institutions, and means of social control, which unites individuals, subgroups and other constituent parts, forming a collective that is capable of lasting and developing" (1970, 239). This definition brings irresistible associations with the concept of society, which makes it little wonder that Beck considers the dissolution of social bonds to be synonymous with the dissolution of society. 
the second, it is employed to refer to the identification of the individual with a social group. ${ }^{78}$ Consequently, it becomes the basis for creating social identity. In both cases, social bonds are treated as an individual property rather than a collective one. Moreover, the concept of social bond functions as a technical term rather than a theoretical idea. As a result, it appears to have little explanatory value.

Nevertheless, social bonds were what creators of sociology assumed to be the conceptual foundation for explaining the very essence of society understood as an entity that transcends the individual and cannot be reduced to the actions of particular individuals. The specific bonds that tie us to other people - both living and dead - would facilitate the existence of society and constitute the only reason why humans are unique among social species found in the natural world. In this perspective, social bonds would be an indispensable component of socializing processes.

It was already August Comte who argued that "[n]othing but the bewilderment caused by theological and metaphysical doctrines can account for the shallow explanations of human affairs given by our teachers, attributing as they do to Man what is really due to Humanity. But with the sounder theory that we now possess, we can see the truth as it really stands. We have but to look each of us at our own life under its physical, intellectual, or moral aspects, to recognize what it is that we owe to the combined action of our predecessors and contemporaries. The man who dares to think himself independent of others, either in feelings, thoughts, or actions, cannot even put the blasphemous conception into words without immediate self-contradiction, since the very language he uses is not his own. [...] Without further illustration, the tendency of Positive doctrine is evident. It appeals systematically to our social instincts, by constantly impressing upon us that only the Whole is real; that the Parts exist only in abstraction" (2009, 235-236; emphasis added). This was echoed by Durkheim, who emphasizes that "association is the only thing genuinely social

78 Ralph Dahrendorf and Stanisław Ossowski use the concept in this way. For the latter, social bonds are equivalent to the phrase esprit de corps, which comprises the following: "an approving sense of belonging to a group; preservation of key conventional elements distinguishing the group; shared values; a consciousness of shared interests; and finally, the readiness to put the group's interests first, before personal ones, if such a conflict arises, or at least the conviction that group interests should be put first" (1967b, 153). 
and sociology is the science of association in the abstract" (1981, 1055; emphasis preserved).

What is even more important, for most thinkers of the period the existence of "social instinct" or "association" was not a given, but something that demanded explanation. Therefore, matters pertaining to the existence and shape of social bonds became for them an independent field of inquiry. Answers to the question of what constitutes social bond contained in themselves at least partial responses to the question of what society is and what it is like. This is clearly visible in efforts, made independently by Durkheim and Tönnies, to describe the transformations of social bonds - efforts that could be also considered as attempts at a typology of the dominant forms of sociality. Additionally, these efforts make it visible that for both thinkers the move from one form of sociality to another entails not only the transformation of fundamental social "structures" but also a radical reconstruction of the entire psychic apparatus of individuals.

Founders of sociology viewed social bonds not only as something that ties humans together to form a higher-order whole but also - or perhaps even primarily - as something that transforms people in a way that cannot be reduced to either individual features or properties of emergent structures. This was clearly expressed by Simmel in his analysis of the category of faithfulness: "The external sociological situation of togetherness appropriates the particular feelings that properly correspond to it, as it were, even though they did not justify the beginnings of the relationship" $(1950,382)$. The role of such a feeling is that "by virtue of it, for once the personal, fluctuating inner life actually adopts the character of the fixed, stable form of a relation. Or vice versa: this sociological fixity, which remains outside life's immediacy and subjective rhythm, here actually becomes the content of subjective, emotionally determined life" (386). In other words, the essence of social bonds - and consequently of socializing processes - would consist in the transformation of personal emotions, which are naturally mutable and transient, into more stable moral sentiments.

The concept of moral sentiments appears already in the title of a work produced by one of the fathers of modern economy - Adam Smith (1984). However, the idea that underlies this concept found fullest expression only in the classics of sociology. Comte, who argued that "intellect, as well as social sympathy, is a distinctive attribute of our nature," nevertheless ascribes greater significance to the latter element, whose foundations rest in sentiments. As he puts it, "common experience proves that after all the principal condition of right action is the benevolent impulse; with the ordinary amount of intellect and activity that is 
found in men this stimulus, if well sustained, is enough to direct our thoughts and energies to a good result. Without this habitual spring of action, they would inevitably waste themselves in barren or incoherent efforts, and speedily relapse into their original torpor. ${ }^{79}$ Unity in our moral nature is, then, impossible, except so far as affection preponderates over intellect and activity" (2009, 15-16).

A similar idea was expressed by Durkheim, who argues that "man is twofold. Within him are two beings: an individual being that originates in the organism and whose sphere of action is strictly limited by this fact; and a social being that represents within us the higher reality of the intellectual and moral order that we know through observation - by which I mean society. In the realm of practice, this duality of our nature makes it impossible to reduce a moral ideal to a utilitarian motive; and in the realm of thought, this duality makes it impossible to reduce reason to individual experience. Because he participates in society, the individual naturally transcends himself when he thinks and when he acts" $(2001,18)$. Durkheim was well aware that behind Adam Smith's "bands of love and affection" $80(1984,85)$, which bind members of human societies, a control apparatus is hidden, one that is specific to a given form of society and safeguards social coercion. "In conclusion," Durkheim argues, "social life is nothing other than the moral milieu, or better, the sum of various moral contexts which surround the person. In calling them moral we mean these milieux consist in a complex of ideas: as a result, they are, with respect to individual minds, just as the physical setting is to living organisms" (1981, 1064; emphasis added). Acceptance of these ideas entails active recognizing of others as members of the same "moral universe."

79 It is worth to emphasize here that this claim of the - considered today as entirely obsolete - classic, harmonizes with latest findings on the functioning of the mind, discussed in the previous chapter. Comte devotes an entire chapter of his work to family as the place where individuals are imbued with moral sentiments. A defense of Comte as a thoroughly modern thinker was penned by Norbert Elias (1978), who emphasized that it would be completely unjustified to treat Comte as a forerunner of methodological positivism, because the Frenchman considered the essence of scientific thinking as an inseparable combination of observation and interpretation.

80 "It is thus that man, who can subsist only in society, was fitted by nature to that situation for which he was made. All the members of human society stand in need of each other's assistance, and are likewise exposed to mutual injuries. Where the necessary assistance is reciprocally afforded from love, from gratitude, from friendship, and esteem, the society flourishes and is happy. All the different members of it are bound together by the agreeable bands of love and affection, and are, as it were, drawn to one common center of mutual good offices" (Smith 1984, 85). 
To be sure, the central role of morality in regulating human actions was recognized a long time ago: morality has been explored by theology and philosophy. The former would seek the source and confirmation of morality in God, whereas the latter - in individual reason guided by either the categorical imperative (Kant) or individual interest (Hobbes). The creators of sociology intended to locate this discipline in the space between the above two forms of thinking: it was supposed to emerge as a positive science of morality, which meant searching for natural (not revealed or transcendental) sources and grounding of morality. The crucial novelty of this emerging science was to replace the individual with society as the moral subject. It was processes of socializing, considered primarily as processes of forging social bonds, that were supposed to be the source of moral sentiments felt by individuals.

Of course, the individualization of sociology (described in previous chapters) led to a significant modification of all its classical axioms. Sociology ceased to be a science of society viewed as a specific moral universe forming individual actions, and became a science of society viewed as a system of structures formed by these actions. In effect, the very concept of social bonds was transformed. They were no longer emotional ties that transform human motives, enforcing such courses of action that one would not undertake out of his or her own initiative; instead, they became the kinds of ties that create some structures or individual identities. This also transformed the theoretical status of the discussed concept: whereas in the first case bonds can undergo substantial changes (e.g. they can move from mechanical to organic solidarity, from Gemeinschaft to Gesellschaft), in the latter case they may only exist or not.

It needs to be strongly emphasized at this point that emotional bonding, which lies at the heart of social bonds (as demonstrated in the previous chapter), is by no means a question of "love," "fidelity," "loyalty," "shared values," or other similar positive feelings that should be naturally experienced in relation to other members of the community, as some of the classics argued. The emotional character of bonding is rather founded on feelings of guilt and obligation, which are often in conflict with individual interests, sentiments and predilections. One may hate his or her family and yet feel an internal obligation - indeed, one of moral nature - to help its members. One may harbor a grudge against one's homeland, owing to its treatment of him or her, and yet stand up in its defense if the need arises.

Thus, an analysis of the transformations of social bonds must begin with restoring them to their proper place within the concept of society understood as a moral universe. 


\subsection{The nature of social bond}

It is symptomatic that the conviction about the dissolution of social bonds in postmodern society has led in the contemporary social sciences to the foregrounding of concepts that do not just help in analyzing ongoing transformations, but rather create a certain normative framework, a specific “Design," or telos, whose finding and implementing would restore the lost harmony and order to the social reality. A closer look at the currently most popular areas in sociological research - such as those defined by the concepts of the civic society, social capital, social trust, or even social identity, alongside with Giddens' "pure relations" that lie at its foundation - makes it clear that all of them are based on a mythologized view of community, at the same time calling for efforts to restore it.

However, as Jean-Luc Nancy argues (1991, 23), despite the fact that contemporary thought is "magnetically attracted toward community", it is nevertheless incapable of "thinking community" because it cannot thematize any issues otherwise than by taking as its point of departure the nearly dogmatic assumption about the sovereignty and ontological primacy of the individual subject. In consequence, instead of engaging itself with the search for the true meaning of the sense of community it merely repeats fairy tales about the lost, total community that needs to be recovered. ${ }^{81}$ According to Nancy, it was Jean-Jacques Rousseau who promulgated this "retrospective consciousness of lost community" and expressed the view that it was the emergence of society that created separate individuals, ruining the original intimacy and totality of communal being. Nancy also argues that although Rousseau was the first to articulate this thought, the concept of lost community runs throughout Western history, beginning in ancient Greece and culminating in Christianity, which developed the idea of universal brotherhood, in which the entire humanity would be joined in communion with God.

As Nancy claims, today's widespread "communitarian engineering" - which creates communitarian projects and desires to impose them on all the living entirely disregards the fact that none of the empirically known communities like family, polis, or the early Christian Church ever created such a substantial and total communal identity. "A community is not a project of fusion, or in some general way a productive or operative project - nor is a project at all"; rather, it is, for its members, "the presentation of the[ir] finitude and the irredeemable excess

81 Cf. for example a more detailed analysis of contemporary views on family as a kind of a myth of "a lost paradise" in Christopher Lasch's Haven in a Heartless World (1977). 
that make up finite [human] being: its death, but also its birth" (15; emphasis preserved).

Nancy claims that the two typically modern cults - that of the individual and that of the communal totality - are actually two sides of the same coin as well as a source of confusion, making it difficult to recognize that "being-in-common" is the only model of community. Being-in-common allows us to become conscious of our birth, death and existence as separate beings. It is the fact of common existence that allows individuals to consider themselves as individuals. "Outside of community," Nancy argues, "there is no experience" (21), emphasizing at the same time that "being-in-common is not a common being" (29), it is not a communion of souls united in the realization of a specific goal, or under the banners of a specific idea. "In place of such a communion, there is communication," Nancy writes (28), referring earlier to Georges Bataille, who claims that "[c] ommunication is a fact that is not in any way added onto human reality, but rather constitutes it" (after Nancy 1991, 21) by making our difference present for others, and for ourselves through these others.

What we consider to be the most interesting element of Nancy's argumentation is the attempt to combine commonality with individualism, or rather - to use a more appropriate phrase - with the "singularity" of individuals. For him, the essence of the community does not lie - as has been usually assumed - in the similarity of lifestyles or worldviews. Rather, communities emerge from coexistence, which involves interactions between distinct and different beings. It lies between them, just like the word "and" in the phrase "me and you and them": "a formula in which the and does not imply juxtaposition, but exposition" (29). In other words, "community means $[\ldots]$ that there is not a singular being without another singular being, and that there is, therefore, what might be called, in a rather inappropriate idiom, an originary or ontological 'sociality' that in its principle extends far beyond the simple theme of man as a social being" $(1991,28$; emphasis added). Therefore, community is not established through formal similarity or some need to become like others - it cannot be produced by an act of the arbitrary will.

We have deliberately used the rather archaic term "arbitrary will" - which was employed by Ferdinand Tönnies to describe one of the two types of social bonds he distinguishes ${ }^{82}$ - because Nancy's account of commonality turns out

82 Tönnies himself did not use the concept of bonds, employing the term "relationship." Similarly, Durkheim used the term "solidarity" to describe two kinds of relations he distinguished. In contemporary sociology, both terms are defined as relating to different kinds of social ties. We need to remember that the concepts of Gemeischaft and 
to be unexpectedly identical to what the venerated sociologist claimed to be the meaning of Gemeinschaft. Considering it as synonymous with a community whose members works harmoniously for the common good, contemporary scholars usually forget that the meaning of the concept of Gemeinschaft was, in Tönnies' theory, determined by the idea of "natural will." "Thus, in its origins," he writes, "natural will must be understood as being inborn and inherited" (Tönnies 2001, 97). It is natural in the same sense in which any organism's biological functions are natural. "The exercise of will or desire," he argues, "cannot be taught" (143), just like one cannot learn to breathe, because both "actions" are simply part of our biological nature. As far as natural will is concerned, "[its] underlying motive forces are found in organic life" (100); what provides them with particular directions and forms is "mental life," possible due to communication and finding expression in customs and religion.

Natural will manifests itself in three basic ways. The first one comprises preferences, i.e. affirmative feelings (or repulsion, i.e. negative feelings), formed in relation to outside objects or particular people. They are rooted in organic drives connected with survival, while their full description brings to mind the concept of Damasio's "somatic markers" (1995; cf. also Chapter Two). The second form of natural will comprises forces of habit, which originate in repeatable actions, gestures and experiences. As any other form of training, they lead to the formation and embodiment of action schemes, resulting in the emergence of a disposition to act in a certain way. Finally, the third component of the natural will is comprised by memory, which Tönnies describes as a specific development of the mental apparatus whose vital element is thinking ("the physiological processes that correspond to thinking"). Memory is the condition of abstract thinking, which is in this sense rooted in and dependent on it. In order to emphasize this point, Tönnies quotes Spinoza saying that "[p]eople who believe that they speak or keep silent or do anything by free decision of their own minds are dreaming with their eyes wide open" (108).

According to Tönnies, as an effect of the natural will, the community's existence does not demand from the individual any reflective or abstract realization of the very fact of belonging to it. Insofar as people can identify the whole of which they are part, this whole exists in them, sustained and conditioned by them. Just like "intentionality without consciousness" (cf. Dennett 1996), community "floats in the air" (Tönnies 2001, 64). Individuals adjust to each other

Gesellschaft, introduced by Tönnies, denote both the type of bonds and the type of community created by them. 
or - to put it more colloquially - click with each other in the course of intense interactions; they incorporate the community as their second nature through actions generated by the natural will, i.e. through preferences, habits and memory. As far as this kind of activity is concerned, he argues, "[i]ts purpose lies in itself, not in some outside aim" (166).

Thus, despite the fact that community creates a specific dependency in which "life means mutual possession and enjoyment, and possession and enjoyment of goods held in common" (36; emphasis preserved), this dependency is not an intentional product of its members. It is not the case that individuals can grasp the "rationality" of communal living, and then form a community in order to secure greater benefits. Communities cannot be formed by a decision coming from the domain of an "arbitrary will." They are rather formed by living together - "beingin-common," as Nancy puts it - and emerge from actions whose course is determined by pre-reflective preferences, adaptations and memory. Mutual connections are therefore the product of these actions, just like the joint use of goods, which has nothing to do with the contemporary understanding of the common good.

Let us pause briefly and consider the last issue. The reception of the concept of Gemeinschaft was certainly influenced to a large degree by Tönnies' argument that "the theory of Gemeinschaft is based on the idea that in the original or natural state there is a complete unity of human wills. This sense of unity is maintained even when people become separated" (22, emphasis added). This claim probably provided a basis for those contemporary accounts of the commonality that emphasize the necessity to subordinate individual preferences to the idea of the common good.$^{83}$ However, such "idea" is the result of consideration, whereas for Tönnies - as we have demonstrated - the "unity" of natural will is somewhat guaranteed by the needs and experiences shared by people in a given collectivity. What is more, it has an organic character insofar as "the whole is not merely the sum of its parts; on the contrary, the parts are dependent on and conditioned by the whole, so that the whole itself possesses intrinsic reality and substance" (21). By opposing a mechanistic approach typical for physics and related disciplines, which splits such wholes into a multitude of "atomic particles," Tönnies declares that atoms are "somethings which are nothings, or nothings which are somethings" (20). One could argue along the same lines that the individual abstracted from an organic unity does not really exist outside such

83 As Will Kymlicka demonstrates (2002), this is a characteristic feature of communitarianism, which Nancy criticizes. 
community. It is only inside the community that s/he acquires her/his proper meaning; therefore, every individual becomes indispensable. It is their complementarity which contributes to the unity of the "organism," i.e. community.

Community means mutual dependence among members of this type of organic unity. Rationality, on the other hand, being the effect of consideration and separating desire from action, is the domain of the arbitrary will. It constitutes itself on the basis of the individualization of acting people. Arbitrary will grows out of the natural one "through a concentrated effort of thought which is constantly extended and renewed" (Tönnies 2001, 114). Thanks to this intellectual effort, preferences are transformed into goals, memory yields to consideration, while habits are subjected to decisions. In other words, thinking sets up a space between impulses or drives which originate in the "natural," biological component of human beings and their actions. This allows to reveal the arbitrary will - the will sensu stricte, in its contemporary understanding. This is also the way in which Weber's rationalization of action manifests itself.

One needs to keep in mind that for Tönnies the transition from the natural to the arbitrary will - the two terms could be treated as shorthand for changes in the functioning of the psychic apparatus, or in the general attitude towards reality $^{84}$ - is linked to the transformations of the fundamental framework of social organization. "Thus, the natural will of the individual generates from within itself the abstract way of thinking and rational calculation which tend to break it up and make it dependent on these new forces. In the same way, we observe among historic peoples the development process of market Society and the system of market rationality evolving out of the original Community-based forms of life and will. In place of the culture of the people we get the civilization of the state" (249-250). The existence of this kind of connection suggests that the currently observed changes in forms of social organization should be accompanied by the emergence of a new type of the "will."

In contemporary sociology, especially in its variants that focus on postmodern society, we encounter many ideas supporting the above conclusion. Giddens' "pure relations," Habermas' "actions aiming at coming to an understanding," and the fundamental assumption of the interpretive paradigm, namely, the claim that the social order is created in the process of symbolic interactions - all convey, either implicitly or explicitly, the belief that social reality is, or could be, constructed reflectively through dialogue. One could also say that whereas the

84 Similar changes were described by Weber as the process of rationalization of actions accompanying transition to modernity. 
consideration underlying the arbitrary will is, in a sense, derivative of action (being rooted in experiences of particular individuals), the concept of reflexivity, which contemporary theories invoke, entails much more, involving continuous monitoring of one's identity, actions and social reality, which would be carried out in abstract terms derived from the symbolic realm or "expert systems."

Following this idea and adhering to Tönnies' wording, one ought to say that the transition from modern to postmodern society is accompanied by the emergence of a new type of "will," in this case one developing out of the "arbitrary will." This type of will - let us call it "reflexive" in reference to Giddens' discussion (2006) - would involve a transition from consideration to reflection, from decision-making to developing strategies of action, and finally, from goal to project. Its fundamental function would not be to make behavior more rational, but rather to increase its authenticity, its relevance to the abstract project of identity, and to reach agreement in dialogue with others. ${ }^{85}$

The concept of identity, which emerges in many theories discussed here, is what links the various changes in the functioning of the psychic apparatus, described by Tönnies and further discussed in accounts developed by contemporary scholars. This is best exemplified by Jürgen Habermas (1979), who associates the transformations of social identity with the transformations of "institutionally embodied structures of rationality," underlying different "stages in the development of social integration." Habermas invokes the theory of moral development proposed by Lawrence Kohlberg (1981), which is well grounded in empirical research, assuming that there is a compatibility between the occurrence of three different patterns of solving moral problems in onto-genetic development (pre-conventional, conventional and post-conventional) and the "social evolution of moral and legal representations" at the level of society. At the same time, he emphasizes that "the reproduction of society and the socialization of its members are two aspects of the same process; they are dependent on the same structures" $(1979,99)$.

According to Habermas, the most primordial form of identity is the "natural identity," in which the "I" has primarily a physical dimension. Its basic parameters are sex, age, and physical appearance. It is the identity of the organism whose internal integrity is based on the distinction between the "I" and its surroundings.

85 In Habermas' theory of communicative action (1987a; 1987b), real action is a test verifying whether consensus has been reached; however, it does not create the possibility of finding that consensus. Similarly, shared knowledge, necessary to initiate dialogue, is conceived of in conceptual categories, not in categories of sharing certain experiences. 
The transition to modern society, in turn, entails the emergence of "role identity." Individuals assimilate the general, symbolic nature of basic social positions, first in their families and peer groups, later in broader social circles, but physical or material qualities still remain a part of the symbolic definition of roles. Finally, the contemporary stage of development, characterized by great intensification of individualization, is distinguished by "ego identity." It involves the ability to conceive of oneself as an autonomous being, presenting oneself as independent from one's direct surroundings, and to retain continuity, stability and credibility in terms of behavior in situations of conflict and in the face of contradictory expectations regarding the roles that individuals are supposed to play. At this stage, the individual acquires "free access to the interpretive possibilities of the cultural traditions" (93).

It is easy to notice that the transition from natural identity to role identity and then to ego identity, considered as resulting from the transformations of structures of rationality, corresponds to the transition from the natural will to the arbitrary and then the reflexive will. Fig. 3.1. provides an overview of the relationships between these concepts.

This part of Tönnies' argumentation, which combines the two types of the will with different types of ties that connect individuals, could be supplemented in a similar way. Insofar as he ascribes the natural will to community, and the arbitrary will to society, one could expect that the reflexive will would correspond to another type of collectivity. According to what is pointed out in contemporary sociological literature, it is network systems that would be most apt to play this role. In this way, we would obtain a concise record of the process in which the forms of social order change - one that would combine the transformations of psychic apparatus with the transition from traditional society, through modern one, to postmodern network society (cf. Castells 2001a). In order to complete it, one would simply need to align types of social order with types of attitudes towards reality (the "will"), adding to the scheme something that would correspond to the dominant modes of socialization and social control - two factors that form the "moral sentiments" that lie at the foundation of social bonds. It would be possible to adapt for this purpose the well-known division into cultures of shame and cultures of guilt (Benedict 2005, 222-223), supplementing it with the "culture of authenticity," which has taken its toll today.

The scheme obtained in this way (cf. fig. 3.2a.) has one undeniable advantage: it provides a synthetic description of the co-variance of three fundamental categories of variables used in sociology to analyze the functioning of society - structures from the systemic level, individual actions, and mechanisms that guarantee the sustainment and reproduction of society. However, its 


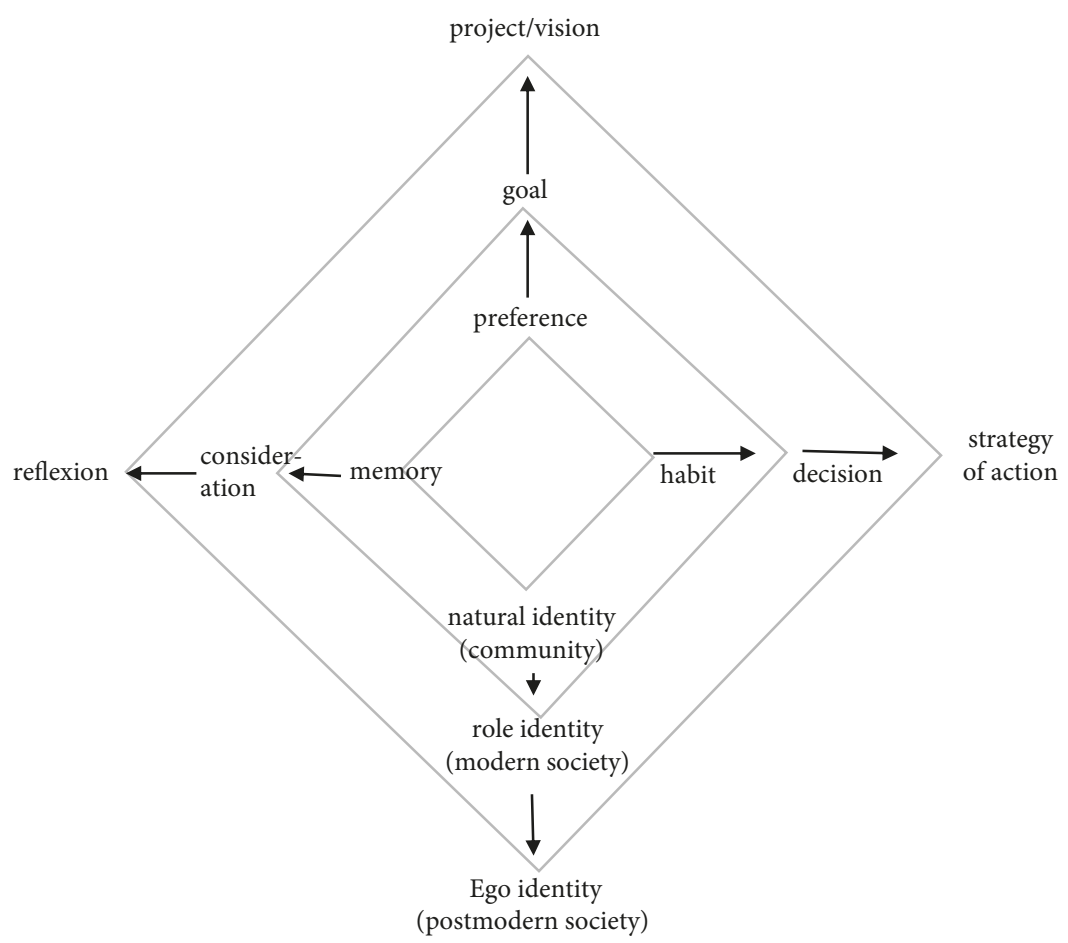

Fig. 3.1: Hypothetical transformations of individual "psychic apparatus" and its positioning with regard to the community

fundamental disadvantage is that it unambiguously imposes a vision of progress, both of people and societies. This progress would be a process in which humans break the "shackles" of both nature and culture (it is the "liberation" from cultural norms that supposedly guarantees authenticity), reaching ever higher levels of self-reflection and fulfillment, while society itself achieves ever greater degrees of complexity and progress (whatever these terms might actually mean). ${ }^{86}$

86 Let us draw attention to the fact that the idea of self-realization, particularly underscored in humanistic psychology and appropriated into mass culture in the form of "being authentic," collides - in a rather amusing way - with the "naturalness" ascribed to members of primitive communities. The assumption made by humanistic psychologists, namely that every person is equipped with a certain potential to develop, and that culture merely thwarts and enslaves us, best illustrates the conviction, 


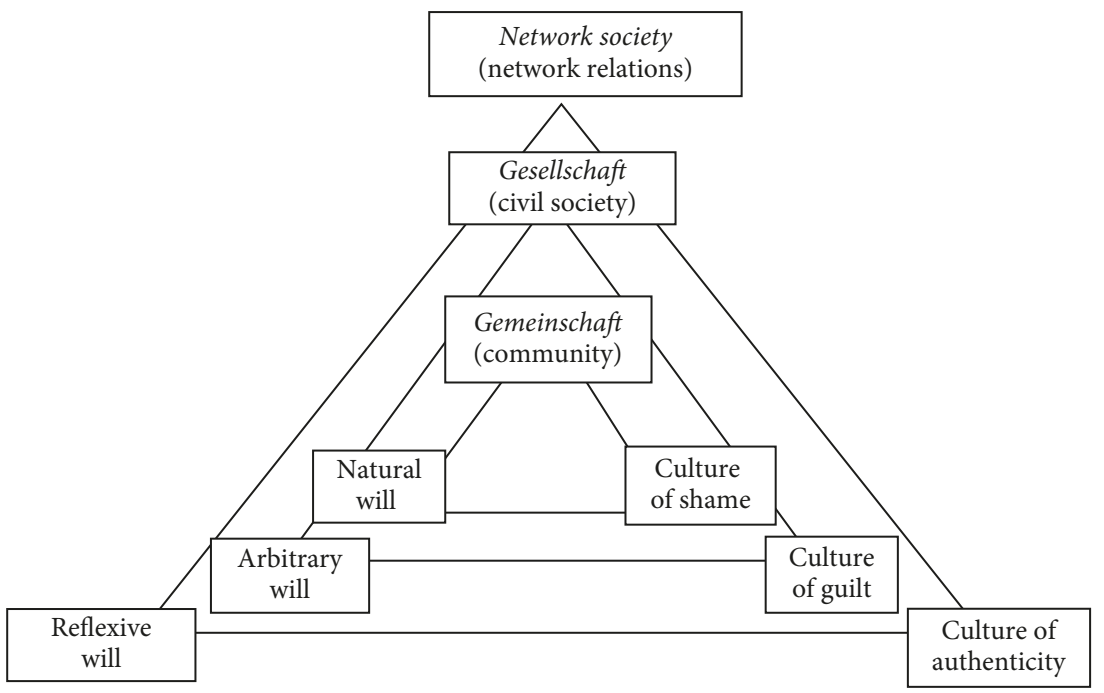

Fig. 3.2a: Hypothetical co-variability of types of social order, types of the "will," and types of culture

This is exactly the kind of vision we protested against in the previous chapter; moreover, it contradicts the assumptions made by Tönnies, for whom both the concepts of the natural and the arbitrary will, as well as those of Gemeinschaft and Gesellschaft, constitute tools for describing various types of social ties, and

widespread in social sciences, about the ontic primacy of the individual over the society. This reveals an important crack, especially in sociology, because it remains unclear what the individual's "authenticity" would be based on if it is assumed (as is done widely in this area) that the "producing of a human being" occurs in the process of socialization. Similarly, one could undermine the concept of "network society" as an entity characterized by greater complexity than primitive communities. In our view, the term "network society" (developed by Castells) unjustifiably limits the concept of the network to a specific system of relations that begins to emerge in contemporary society. This makes it more difficult to discern that communities and societies also create networks of relationships, only of different properties. However, to do justice to Castells, it is worth underlining that his concept certainly contributed to an increased interest in network analysis, an immensely interesting approach, one of whose key representatives in sociology is Barry Wellman (cf. e.g. Wellman \& Wellman 1992). 
not processes of society's evolution.$^{87}$ As Tönnies claims in one of his later works, he knows "no state of culture or society, in which the two elements, Gemeinschaft and Gesellschaft, would exist in separation" (after Szacki 2002, 445).

We emphasize the typological character of Tönnies' theory not only to render his intentions faithfully but also to express the conviction that his concepts would be far more useful today if we were to treat them as a means of describing distinct forms of ties that manifest themselves in human relationships. Indeed, what we see as Tönnies' greatest contribution is his indicating of the fundamental difference between the ties characteristic for communities and the ties that underlie other forms of human association, as well as his drawing attention to the nonreflexive and (in this sense only) "natural" character of mental processes that accompany the communal kind of relationships among people.

However, in order to make use of current knowledge for analyzing consequences entailed by such a conceptualization of the nature of communal ties and their later transformations, we need to examine the process that lies at the foundation of the above-mentioned changes in the functioning of psychic apparatus, which - as could be inferred - should in turn affect the transformations of social bonds. By this we mean here the process of changes in human thinking. After all, in the light of conclusions drawn in the previous chapter, the "natural will" is not as "natural" as Tönnies assumed ${ }^{88}$ Nor is it possible to consider the development of thinking, which sparks the transformation of this will, as a spontaneous product of the unique properties of the human mind. Dennett's concept of mind, adopted in this book, rests on the assumption that human intentionality and intelligence are not the direct products of our genetic equipment, but a function of the use made of culture by particular individuals. This means that,

87 It has to be noted right away that he was not entirely consistent in his considerations of the nature of this distinction (cf. Szacki 2002, 444-446). In his best-known work (2001), the "emergence" of the arbitrary will from the natural one clearly has some properties of a developmental process, just like Gesellschaft's domination following that of Gemeinschaft. However, in his later works Tönnies clearly declares that both pairs of terms constitute ideal types of basic social relationships, and it is this statement we take to be binding here. We shall return to the problems arising from mixing historiosophy with typology towards the end of the chapter.

88 Both individual tastes and habits, let alone memory, are a complex product of socializing processes and have nothing to do with naturalness in the biological sense. In this respect, Tönnies was much more influenced by nineteenth-century views on humanity than his peer Marcel Mauss, who was able to demonstrate in his essay on "techniques of the body" that even our physiological reactions are culturally conditioned. Cf. Mauss 1973. 
if we wish to fully grasp the transformations in thinking indicated above, we have to closely examine both the concept of culture, and the factors that shape its "use."

\subsection{Culture as a "toolkit"}

In social sciences, we encounter two competing and contradictory accounts of the relationship between humankind and the culture it has created. The first emphasizes the weakness of human beings, which (as distinct from animals) would be deprived of any instinctual basis for behavior, making them defenseless in contact with threats posed by the natural environment. In this account, culture is considered to be a prosthesis that supplements the biological "imperfections" of humanity. ${ }^{89}$ In the second view, culture is considered primarily as evidence of human magnificence, a living proof of the incredible qualities of the human brain, which has proven capable of producing "plasma physics, Picasso and pizza," to recall once more the words of Stringer and McKie (1996). Whereas the former view entirely disregards the fact that humankind was managing quite well without culture for thousands of years after becoming fully developed in biological terms, the latter passes over other human "inventions" like civil wars, concentration camps or racial segregation.

Both approaches can be found within sociology. Adopting one of them usually depends on whether the question of culture appears in the context of socialization processes, or that of explaining human behavior. Generally speaking, however, the place of culture in strictly sociological analyzes remained marginal for quite long. As has been emphatically stated by an eminent culture scholar Robert Wuthnow, "[a]lthough it is possible to point out specific studies in sociology that have contributed greatly to the understanding of culture, the discipline as a whole has not given particular prominence to the importance of culture. In fact, culture often appears in empirical studies as a vague concept to which relatively superficial attention is given or as an outdated form of explanation that must be superseded by factors of greater objectivity and significance" $(1987,2)$.

Importantly, the most widespread understanding of culture found in sociology has been the one that refers to values and norms, beliefs and attitudes. Ann Swidler (1986) associates it directly with the tradition initiated by Weber,

89 This human quality is viewed by Gehlen as the source of humanity's originality and its special status in the natural world. In his words, "given their innate physical construction and shortage of instinct, humans dealing with raw nature, like animals, would not be able to live by any means" $(2001,37)$. 
emphasizing that although he saw interests as the main motivation to take action, he would view the goals and means of such actions as "ideas" defining social values. She also indicates that Weber's understanding of the role that culture plays in shaping actions was fundamentally modified by Talcott Parsons, and in this later form adapted by American sociology. "For Weber's interest in the historical role of ideas, Parsons substituted global, ahistorical values. Unlike ideas, which in Weber's sociology are complex historical constructions shaped by institutional interests, political vicissitudes, and pragmatic motives, Parsonian values are abstract, general, and immanent in social systems" (Swidler 1986, 274). Therefore, they could be incorporated into Parsons' theory to serve the function of integrating the said systems.

The processes of individualization of sociology, described in the first chapter, did not fail to affect the concept of value, linking it directly to individual actions. ${ }^{91}$ Ann Swidler is right to observe that one of the reasons for the continuing popularity of such explanations can lie in the intuitively self-evident assumption made in our culture, which consists in the belief that human actions are organized in accordance with the "means-ends" scheme. ${ }^{92}$ In this account, "[c]ulture shapes action by defining what people want" $(1986,274)$. The popularity of this explanatory scheme has been long-standing, despite analyzes demonstrating that people rarely have the occasion to behave exactly as they wish to, that cultural values are often socially contested and interpreted in various ways, and finally, that sharing values does not necessarily have to lead to similar actions, or even have any direct relationship with them (Swidler 1986; cf. also Marody 1996, 2000b; Giza-Poleszczuk et al 2000).

90 One could add that most of world's sociology has followed this model. This is particularly true of Polish sociology, which developed clearly under the influence of the American tradition, and is to a large extent a "sociology of attitudes and values."

91 Robert Whutnow sees this an example of how contemporary sociology has come to be dominated by subjectivity, because in this discipline "the fundamental elements of culture are not mental constructions, created or adopted by individuals; and [because] they represent, are rooted in, express or indicate subjective individual experiences such as opinions or fears" $(1987,11)$. It is worth noting here that Wuthnow attributes the subjective approach not only to the research that refers to attitudes and values as the fundamental component of culture but also - and perhaps even primarily - to all analyzes based on the assumption of the symbolical construction of social reality.

92 Let us note on the margin here that this premise corresponds with Tönnies' assumption regarding the dominance of arbitrary will in the modern society. 
The inadequacy of interpreting human behavior in terms of ends and means becomes even clearer when the problem is considered from a longer time perspective. The claim that the culture created by the Cro-Magnon shaped their actions by defining what they desire seems absurd. Doubtless, even if we disregard the issue of the development of intentionality, the very conditions created by the period's natural environment (a completely "un-socialized" habitat, let us note) subordinated Cro-Magnon's "desires" to the fundamental struggle to survive. ${ }^{93}$ In other words, assuming a longer time perspective has convincingly revealed the historical "locality" of the analyzed scheme and its underlying vision of individuals as reflexive beings who choose their goals from among a culturally sanctioned catalogue of values, and who consider rationally which means should be employed to achieve these selected goals. However, even in the case of contemporary humans this account does not embrace the full spectrum of actions, whose "goals" are, after all, also defined (in some cases predominantly so) by various moral constraints stemming from a sense of responsibility, and by existential pressures determined, on the one hand, by human needs, which have a biological basis but are in fact already socialized to some extent, and on the other hand - by the means of taking actions, available to particular individuals and collectivities.

The last factor is particularly emphasized in the theory developed by Ann Swidler, who notes that "culture is not a unified system that pushes action in a consistent direction. Rather, it is more like a 'tool-kit' or repertoire [...] from which actors select differing pieces for constructing lines of action” (1986, 277; emphasis added). In this perspective, taking action is explained in terms of strategy, or "a general way of organizing action [...] that might allow one to reach several different life goals" (277) - not by considering individual forms of behavior from the perspective of how they match specific means and goals. As she argues, "[i]f culture influences action through end values, people in changing circumstances should hold on to their preferred ends while altering their strategies for attaining them. But if culture provides the tools with which persons construct lines of action, then styles or strategies of action will be more persistent

93 Survival of individuals and the species as a whole involves reproduction and related activities. Of course, this does not preclude the existence of other factors motivating the actions of our distant ancestors (cf. Marody 1991), but gives them a different meaning by linking them with the principal striving to survive. It is worth mentioning that the desire to survive is not treated here as equivalent to the "fight for survival." For more on this subject see: Sahlins 2006. 
than the ends people seek to attain. Indeed, people will come to value ends for which their cultural equipment is well suited" (277).

Swidler also underlines that the impact of culture may take different forms depending on whether it occurs in a period of stability, or one of social change. ${ }^{94}$ In the former case, culture reinforces and supports strategies of action developed by distinct social groups, whereas in the latter, culture itself becomes the source of new strategies. In the former, culture's influence materializes primarily through the impact of tradition and common-sense knowledge, whereas in the latter - through ideology, or "explicit, articulated, highly organized meaning systems (both political and religious) [...] aspiring to offer a unified answer to problems of social action" (278-279).

Drawing attention to the inconsistent or even incoherent character of cultural contents also seems to be an achievement creditable to Ann Swidler. As she remarks in the article quoted above, "all real cultures contain diverse, often conflicting symbols, rituals, stories, and guides to action" (277). This perspective radically departs from the dominant trend in sociology, which emphasizes the coherence of culture and its power to integrate a given collectivity. ${ }^{95}$

One particularly important element in Swidler's theory is how she underlines the active attitude assumed by individuals in relation to culture. "Realistic cultural theory," Swidler writes, referring (among others) to Denis H. Wrong (1961), "should lead us to expect not passive cultural dopes, but rather the active, sometimes skilled users of culture whom we actually observe" (277). Let us immediately add - in reference to the theories of Mead and Dennett - that for such users the tools are not only something that is stored in culture and can be utilized by reaching out for it, but also constitute the fundamental form of the mind's "equipment," its fundamental mode of existence, outside of which it would lose all of its specific qualities characteristic for people only. ${ }^{96}$

94 Although this distinction is explicitly introduced, it has not been made clear. The author uses descriptive terms ("settled vs. unsettled lives"), without commenting on what makes the two types of life different, and what mechanisms cause them to become "disorderly." The text contains references both to social transformations (278), as well as to orderly and disorderly "cultural periods" (281).

95 Despite being one of the most frequently quoted definitions in contemporary sociology of culture, the conceptualization of culture proposed by Swidler has not become popular in other areas of the discipline so far. It is a pity, because it could decidedly influence the understanding of relations between culture and humanity.

96 For accuracy's sake, we should note that this link is not found in the theory of Ann Swidler, who does not explicitly mention where individual users would derive their skills from. Even more importantly, she does not discuss the sources of the human 
This way of conceiving the relationship between humans and the culture they create and use immediately leads to another problem, analogous to the one we dealt with when considering Mead's assumption regarding the social framework of shaping the mind, analyzed at the level of processes of anthropo- and sociogenesis. If human mind derives its unique abilities solely from culture, i.e. if we deprive it of any genetically-programed "surplus" competences, then even assuming the "foundational holism" hypothesis could not explain the source of its development and, consequently, the source of societal development, or - if we prefer to avoid the evaluative connotations contained in the word "development" - the source of socio-cultural changes. ${ }^{97}$ After all, "active users of culture," could also include other gregarious animals: not only primates but also ants or bees, to recall examples referred to earlier; however, their activity does not lead to any fundamental changes in their "culture."

However, the above comparison allows to assume that the source of difference between the functioning of people and other gregarious animals should be located primarily in the more specific properties of the "peripheral devices" that aid the human mind and simultaneously act as basic vehicles of social memory, exteriorized and accumulated in human culture. Taking into account the earlier discussion of mechanisms that underlie the emergence of specifically human forms of intelligence and social organization, one could also assume that there are three basic kinds of such "devices": language, tools sensu stricto and

mind, restricting her argumentation to the framework defined by an individualistic perspective that dominates in sociology. However, it is possible to link the theme of culture as a "toolkit," which she develops, with Dennett's views on the functioning of the mind. Suffice it to recall one passage from the article quoted above, in which she claims that "people will come to value ends for which their cultural equipment is well suited" (1986, 277).

97 This problem is usually articulated in contemporary sociology as one of relations between the level of individual actions and that of social structures. It is "solved" by ascribing agency solely either to autonomous actors or social structures. In the "individualizing" approach - regardless of its underlying vision of the individual as equipped with a unique mind - it remains unresolved how social structures emerge from individual actions. In the "structural" approach, in turn, it remains equally problematic to determine how social change is possible. Although such a change is experienced on the empirical level, it cannot be logically explained within the framework of assumptions regarding the reproduction of social structures through individual actions. It is usually explicated by referring to factors operating outside the entire system. Cf. a more detailed discussion of this phenomenon in Marody 2000. 
established schemes of action, which are "embodied" in human habits. Let us consider them one after the other.

\subsubsection{Language}

From among the three above carriers of culture as social memory, it is language that has attracted greatest attention. We shall not even attempt summarizing what has been written by various scholars on the subject of its properties in the context of processes that have shaped human thinking and gave it its specific character. A survey of such investigations can be found in the work by Berger and Luckmann, who emphasize first and foremost language's ability to communicate contents that are not given "here and now," which causes, first, that it is "capable of becoming the objective repository of vast accumulations of meaning experience, which it can then preserve in time and transmit to following generations" (1991, 52; emphasis added); second, that it "bridges different zones within the reality of everyday life and integrates them into a meaningful whole;" and third, that it "is capable of transcending the reality of everyday life altogether" (54), thus facilitating the creation of a symbolic universe.

Fully accepting the basic functions of language listed above, we nevertheless wish to draw attention to the omission of the process of language's historical development. ${ }^{98}$ This makes it difficult to grasp the influence exerted by language transformations on the emergence of more specific characteristics of this "device," and consequently on the information it produces and transmits. There are at least three properties lying at the roots of these transformations, which introduce a fundamental difference between human and animal communication. The first two have their origin in language's immanent properties, while the third is a derivative of the changes that have occurred in the area of technology.

The first property is usually described metaphorically by claiming that the language of animals is based on "sentences," whereas the human one - on words (cf. Bronowski 1979). In the basic, task-related function, both human and animal communication serves primarily to coordinate individual actions by joint

98 It is not an accusation against the theory developed by Berger and Luckmann (whose significance cannot be overestimated), but merely an observation: they were not interested in that aspect of the functioning of language. Anyway, it is neglected by most researchers who analyze the properties of language as langue, disregarding the fact that its functioning as parole ought to account for those of its properties which - like lexical changes or shifts in the meaning of some words - are an effect of the historical evolution of everyday spoken communication. 
attention to specific elements in the natural environment (cf. Tomasello 1999). However, sounds made by animals ${ }^{99}$ form the entire message which contains a usually general indication of both the object and the action that ought to be taken with regard to it. Breaking down such holistic messages into individual words allowed humans not only to achieve greater precision in directing attention by being more specific in relation to objects and dissociating them from actions but also - and perhaps primarily - to create the potential for "wresting concepts from their interwoven connectionist nests and manipulating them" (Dennett 1996, 159).

It was precisely this ability to manipulate the ever more precise internal representations of the outside world that lay at the basis of processes that made human language such an effective tool: not just for storing information but also for creating it. ${ }^{100}$ Therefore, one could assume that the distinguishing of words in the human system of communication constituted the first step towards the emergence of more complex forms of reflexivity. It would focus on relations between particular objects and states of the external reality (which, in turn, necessarily contributed to the development of the specifically human ability to treat oneself as an object of examination), and on the very essence of concepts considered in separation from any particular objects they represent or actions taken in relation to them (which in turn contributed to the development of science).

The second important step was to functionally dissociate the purely factual contents of conceptual representations from emotions that could be associated with them. Jacob Bronowski draws attention to this, when he argues that "[while communicating] animals do not pass any information. What they communicate are instructions" $(1984,43)$. In the language of vervet monkeys, the call "Leopard!" (1984; cf. footnote no. 1) is unambiguous and cannot be interpreted in any other way than as a signal calling for a specific set of actions. If uttered by a human, this call would become information since it could have multiple

99 It is worth mentioning that animal systems of communication do not have to be sound-based only. Bee dance is a good example of a precise "language" used to communicate information about sources of nectar.

100 Hedgehogs can "remember" that apples fall in certain places. However, due to the kinds of representations that dominate in the functioning of their brain, they cannot ask themselves how it happens that apples fall on the ground (and not the other way round) because they lack distinct words to denote all of these "objects." We do not intend to suggest that at the basis of Newton's physics there is only manipulation of words, but rather wish to demonstrate the potential inherent in the mere change of word order in a sentence, not to mention more advanced forms of processing concepts. 
possible interpretations depending on additional factors shaping the reception of its content. ${ }^{101}$

Two consequences of this characteristic of human language ought to be emphasized here. First of all, the use of language for giving direction to human action - both on the social level by establishing what we call value, and on the interpersonal level by the use of various techniques of exerting verbal influence - demands secondary association of certain emotions with concepts/words (Damasio 1995). Furthermore, the secondary association of emotions with linguistic elements does not really have any limitations - any word can acquire a positive or negative connotations, which allows generating an infinite number of further meanings. Let us draw attention to the fact that this characteristic enabled both the rise of different ideologies, which are based on different evaluations of factually identical phenomena, and the emergence of science, which demands becoming a neutral observer (cf. Elias 2007).

Finally, the third peculiarity of human communication is the emergence of writing as the basic means of storing linguistic messages. Preserving speech in writing creates completely new possibilities for the reception and analysis of messages. On the one hand, this contributes to the emergence of new types of intellectual competence (Goody 1978; cf. also Marody 1987). On the other hand, it fundamentally alters the character of stored information. In societies using oral communication, the only option for preserving individual innovations or intellectual discoveries was to incorporate them into the group's oral tradition, which often depended on non-cognitive factors. With the advent of writing, individual intellectual discoveries were given a greater chance to survive and stimulate other people's cognitive processes. Expanding knowledge became independent of contextual circumstances and political or economic pressures. Stored information can be re-interpreted many times, depending on the changing context. We should add, however, that its physical survival does not predetermine its social impact. The last element relies on the noticing and "activating" of the message kept in the storehouse of culture.

Whereas the first two steps, which furnish human language with its specificity, necessarily belong to the early stages of language development, the third

101 As Bronowski indicates, interpretation depends, for example, on those properties of the sender that are known to the recipient of the message. If the cry "Leopard!" is raised by a person we know to be timid and inclined to exaggerate, it becomes more likely that we would underestimate it, in contrast to the situation in which such a call would be made for example by a safari guide. 
one is related to the transition from oral culture (which relies on the oral preservation of information important for the community) to written culture. In the latter, words cease to be directly related to actions, and can themselves become objects to be acted upon. This is a fundamental change, one that leads primarily to a thorough remodeling of language's function within human systems of communication. This was already observed by Bronisław Malinowski, who expressed the need to "realize that language originally, among primitive, non-civilized peoples was never used as a mere mirror of reflected thought. The manner in which I am using it now, in writing these words, the manner in which the author of a book, or a papyrus or a hewn inscription has to use it, is a very far-fetched and derivative function of language. In this, language becomes a condensed piece of reflection, a record of fact or thought. In its primitive uses, language functions as a link in concerted human activity, as a piece of human behavior. It is a mode of action and not an instrument of reflection" $(1936,312)$.

What is more, in order to facilitate the transition from "mode of action" to "instrument of reflection" - to use the terms proposed by Malinowski ${ }^{102}$ - it was necessary to create a meta-language allowing, among other things, to analyze utterances on the level of "mode of action." In other words, the emergence of reflectivity, as it is understood today, had to be preceded by the development of a "higher-order intentionality" (Dennett 1996, 121; cf. also Marody 1987, 271284 ), which in turn has at its basis not only the gathering of new experiences in the course of developing social praxis but also a fundamental restructuring of the said "peripheral device" of our mind - language.

It is important to stress that all these changes in the social dimension took place as part of long-lasting processes, ${ }^{103}$ whereas in the individual dimension they have always required specific training. It is because features of the

102 This transition would correspond to the one distinguished by Tönnies, namely the transition from the natural to the arbitrary will. The "naturalness" of the former manifests in this perspective not in the lack of thinking, but rather in the lack of its "psychologization" (to employ Norbert Elias' term). For more information on the development of the latter skill see: Elias 2000, especially Part Four.

103 Jack Goody locates the beginnings of the development of a "writing mode of thinking" in ancient Greece, emphasizing that the invention of writing was a factor that created merely the technological possibilities of developing a new style of cognitive functioning, and that in order to initiate this development a series of additional conditions had to be met - social, political and economic ones - which happened for the first time in the Greek society. Cf. Goody \& Watt 1972. 
"language of action" are somewhat genetically coded in our brains. ${ }^{104}$ Features of the "language of reflection," in turn, are the effect of using a particular tool (i.e. writing) and their development is subject to rules specific to the second vehicle of culture: tools in the strict sense.

\subsubsection{Tools}

The role of tools in the development of certain features of the human mind is discussed to some extent in Chapter Two. Following Guille-Escuret, the moment when "language was combined with technology" served as the key stage in the process of anthropogenesis. This moment - which marks the transition from tools based primarily on the use of slightly modified natural objects (stones, bones, branches, etc.) to proper tools that are products of complex processing and contain an "intentional aim" defining their specific use in specialized contexts was certainly genetically conditioned by earlier anatomical changes (lowering of the larynx, enlargement of the brain) linked to the biological evolution of the human species. However, this fact does not provide solid grounds for inferring on its basis that historical development is accurately captured by the "March of Progress" image. Even if we disregard Guille-Escuret's hypothesis (ultimately, it is only a hypothesis, and others could be formulated as well), it turns out that the vision according to which subsequent generations of Humanity face an unwelcoming natural environment and utilize properties of the Mind to invent ever better tools to fulfill their specific needs and coincidentally enslave Nature, does not really take into account at least two important and well-known facts.

First, the discussed theory does not fully explain the fact that there still exist societies that use only Stone Age tools. Nor could their members be attributed with any mental deficits - after all, they can actually use even the most complicated tools after proper training. Second, it does not account for the well-documented fact that most inventions we consider revolutionary from a historical perspective did not actually arise from any, even most general human needs. They were either rejected by a given society, at least initially, or never

104 This is indicated, first, by the fact that human children have no trouble mastering this immensely complex activity, and second, by the fact that the "restricted code" identified by Basil Bernstein (1971; a counterpart of the "language of action") is a code to which we all have access, whereas the "elaborated code" (a counterpart of the "language of reflection") can be found primarily among families of people who have at least high-school education. 
found "proper" use in it. ${ }^{105}$ As Jared Diamond has indicated, most typically, when a given "device had been invented, the inventor then had to find an application for it. Only after it had been in use for a considerable time did consumers come to feel that they 'needed' it;" ultimately then, he concludes, "invention is often the mother of necessity, rather than vice versa” (1999, 242-243).

Therefore, one could say that if some contemporary people are entirely satisfied with Stone Age tools, and if hunter-gatherers, whose brains did not differ in any way from those of today's people, did not use them to improve their "technological equipment" for thousands of years, then the later accelerated development of tools should not be linked with genetically-programed properties of the human mind, but rather with external factors. This is also the conclusion reached by Diamond, who indicates that the decisive moment in technological progress was the adoption of a settled lifestyle, which entailed replacing gathering with agricultural production of food. A settled way of life facilitated the gathering of possessions. ${ }^{106}$ At the same time, accumulation of food surplus led within each community to the emergence of specialists developing currently existing tools and inventing new ones. ${ }^{107}$ The development of technology was also crucially affected by the exchange of information between particular collectivities, which is indicated by the fact that the post-Pleistocene acceleration of technological progress was most rapid in Eurasia - an area characterized by the lack of any major natural barriers, and inhabited by numerous competing communities (Diamond 1999, 399).

The fundamental factor supporting this exchange of information had to be the circulation of tools themselves, as well as other objects produced in individual communities. This is related to two characteristics of such objects, which allow treating them as carriers of information, in turn developing human intellect. The first stems from the fact that many tools contain encoded information about the intentions of their makers, while all tools offer diverse affordances. When we take an unknown object into our hands, its very shape and design suggest

105 The most famous and widely quoted example is the discovery of gunpowder in China, where it was used solely for making fireworks, or the discovery of wheel by the Mayas, who used it solely in kids' toys and not for transportation.

106 Perhaps, it would be an exaggeration, but one could thus say that this was a moment when the "spiral of needs" began to spin, resulting today in thousands of objects presented by marketing specialists as absolutely necessary in our lives.

107 This was part of a broader process that also led to the emergence of "specialists in thinking," which is discussed in Chapter Two, where the theory of Lewis-Williams is expounded. 
certain kinds of movements possible to be made with it, regardless of whether it is a hammerstone, a regular hammer or a modern laptop. ${ }^{108}$ The second feature is derived from the first: manipulation of tools in an attempt to answer the question "What is this used for?" leads to the question "What could this be used for?" and to the discovery of new uses. This is the precise meaning of the statement that human intelligence is materialized in tools. It becomes unleashed each time solely through the act of using them, regardless whether such uses comply with the creator's intention or not. One could even say that objects become tools only through their use. Thus, the impact of such "peripheral devices" on individual intelligence would consist in assimilating various possibilities inherent in a hammer into possibilities of the mind.

Such a view of the role played by the use of tools in the development of specific features of human thinking is valuable in itself because it represents an approach that undermines the above-mentioned popular vision of humanity as the crowning of evolution. However, just like general considerations on language's function, this perspective disregards the historical processes in which the said "peripheral devices" of mind are transformed, as well as its impact on changes both in the very character of information accumulated in tools, and in the human potential to assimilate it. Let us draw attention to the fact that, in the case of most contemporary tools, their solely physical manipulation does not necessarily have to lead to an increase in the intelligence of an individual. Except for extreme situations, ${ }^{109}$ we would consider the use of a cell phone in the function of a hammerstone - which is a kind of "examination by use" - as a proof of intellectual limitations rather than a step forward in the intellectual growth of a given person.

Therefore, we once again come to face the following question: "Which elements of the historical chain of technological transformations ought to be

108 Let us draw attention here to the fact that the principle of ergonomics - a chief tenet in today's design of tools and objects of everyday use - reinforces the intentionality of the makers, as coded in the shape. "Appropriate" gestures ought to suggest themselves. Just like an armchair "invites" one to rest, so a laptop keyboard "invites" one to press the keys.

109 A great example of such a situation, as well as the perfect illustration of the relationship between manipulation of objects and generating what Richard Gregory calls "Kinetic Intelligence" (after Dennett 1996, 99), is provided in the film Cast Away, in which the protagonist, stranded on a deserted island, is forced to cope with basic survival-related issues while having at his disposal just a random collection of items found in a package washed ashore. 
considered the most significant?" Attention would be brought here not to their significance for the development of technology, but rather to the presently analyzed processes of social change, and the role played in them (in the past and now) by tools considered as carriers of information accumulated in culture.

To answer this question, it becomes paramount to shift attention away from those who use tools to those who create them. After all, they are the ones who "encode" the original intentionality in the tool, defining the boundary between its intelligent or senseless usage. As Jacob Bronowski strongly emphasizes, "[c]ivilization is not a collection of finished artefacts, it is the elaboration of processes" $(2011,1086)$. He compares the creators of the earliest tools to modern scientists by indicating that at the basis of efforts made by both there lay a search for structures that are hidden in matter. Both would arrive at their discoveries by submitting nature to the use of tools. "The tool that extends the human hand is also an instrument of vision. It reveals the structure of things and makes it possible to put them together in new, imaginative combinations" (1107). The fundamental difference between the inventors of first tools and their contemporary counterparts - the scientists - lies not in the type of actions, but the type of structures to which the latter guide their attention. This is because "the visible is not the only structure in the world. There is a finer structure below it" (1107). It was the interest in discovering this hidden structure that lay at the foundation of modern science, making it a kind of a meta-tool that codes, in a highly specific way, information generated by the use of tools sensu stricto in the course of scientific praxis. ${ }^{110}$

Assuming such a perspective, the basic caesura in the historical course of technological changes would consist in the transition from tools developed by practitioners to tools that are derivative of broadly understood scientific endeavors.

The earliest tools were made with particular tasks in mind - something needed to be cracked, cut, or stored. ${ }^{111}$ Later on, inventiveness was also inextricably tied

110 As Jan Poleszczuk has brought to our attention, the modern cyclotron is nothing more than a kind of a "mega-hammer," whose use on nature brings the same results as the use of an ordinary hammer - it breaks a given element of nature into smaller parts.

111 "I see that process of 'invention' going on," writes Jared Diamond, "whenever I take New Guineans to work with me in an area away from their homes. They constantly pick up unfamiliar things in the forest, tinker with them, and occasionally find them useful enough to bring home." The assessment of usability depends on the lifestyle of a given collectivity - for modern inhabitants of New Guinea, the yellow pencil left by Diamond turned out to be useful "as an ornament, inserted through a pierced ear-lobe or nasal septum" $(1999,246)$. 
to collecting experiences gained from working in the natural environment and upon it, all thanks to observation and attempts to utilize, through trial and error, materials available in immediate surroundings. As Diamond notes, "complex innovations were usually acquired by borrowing, because they spread more rapidly than they could be independently invented locally" (Diamond 1999, 255). ${ }^{112}$ Their later development was made possible by improving already existing tools and technologies, and due to the necessity to solve particular problems encountered while using them. As the history of more recent inventions shows (such as Watt's steam engine, or Edison's light bulb), the "heroic theory of inventions" that ascribes them to lonely geniuses is actually a myth disseminated in stories for children.

The case is different with inventions made since the end of the nineteenth century: "few $[\ldots]$ were the consequence of a steady piecemeal development or improvement of existing processes; the overwhelming majority resulted from new materials, new sources of power, and above all else from the application of scientific knowledge to industry" (Barraclough 1969, 46). Ways of putting them to use often could not be predicted at the time of their discovery. This was the case, for example, with the electricity-generating dynamo (one of today's basic sources of energy), one hundred years later with laser, which is now standard equipment in dental clinics, or with the relatively recent emergence of the Internet. ${ }^{113}$ The history of these inventions best illustrates the positive feedback between using tools and discovering their possible applications.

However, the fundamental difference between inventions made by the practitioners and those rooted in science discoveries lies in the latter's increasing "informatization," which denotes both the augmentation of information contained in the tool itself, and the increase in the volume of outside information necessary to operate it in accordance with its "inbuilt" potential. Surely, it is possible to start a laptop by randomly pushing its buttons; however, from that moment on, further exploration of its uses is made possible only by information provided by the manufacturer in the form of icons or explanations. Generally

112 This is how the wheel has been made popular: it is "first attested around 3400 BC near the Black Sea, and then turns up within the next few centuries over much of Europe and Asia. [...] Other examples of complex technologies that diffused east and west in the ancient Old World, from a single West Asian source, include door locks, pulleys, rotary querns, windmills - and the alphabet" (Diamond 1999, 255).

113 Stanisław Lem aptly put it by saying that the Internet "constitutes the answer to a question that has not been posed yet." 
speaking, an accompanying user manual is indispensable to operate most of today's tools. ${ }^{114}$

In other words, the highly "informatized" tools come with the assumption that their users have previously acquired certain cultural competences, such as reading, but at the same time "hide" most information that lie at the basis of their functioning. In order to efficiently use a computer, travel on an airplane, or watch satellite television we do not need to possess knowledge necessary to comprehend the complex technological processes that underlie their production. Anyway, large portions of such knowledge become difficult to access, even for well-educated people, due to the specificity of the "structures" that contemporary science deals with. This was addressed by Werner Heisenberg, who once remarked that people are now examining such regions of reality that are inaccessible by senses and can be probed only thanks to complicated apparatuses. Thus, we are leaving the domain of sensual experience - the area in which everyday language was shaped. As a result, the language used in physics is now understood only by physicists $(1979,165)$. One could even say that whereas the basis for socializing processes was provided by the "combination of language and technology" (as Guille-Escuret claims), we are now witnessing the divorcing of these two factors.

The increasing "informatization" of tools is accompanied by two other processes that have had a significant impact on the transformation of culture understood as a repository of information. The first one is the rapid development of tools used to store information. This is touched upon in the above discussion of changes in language as carrier of culture, which culminated in the discovery of writing as the basic tool for preserving oral messages. However, this invention only marked the beginning of changes that bore fruit in the "technological" dimension, giving rise to diverse, specialized devices used directly to store information. The emergence of print, photography, phonography, as well as today's $\mathrm{CD}-\mathrm{ROM}$ and computer hard drives, defines subsequent stages in the development of the kinds of tools whose shared function is to store two kinds of information: the one preserved using a particular medium, and the one "recorded"

114 In many cases, instruction manuals are not only meant to provide information to users but also protect the producers from the consequences of using tools in ways differing from their original purpose. One US manufacturer of washing machines was forced by court order to add a warning for consumers that "the washing machine is not suitable for cleaning and drying domestic animals" after a client washed her poodle in the machine and sued for compensation after being unpleasantly surprised by the result. 
indirectly in its form and mode of functioning, as well as in the technology used to make it, and so forth.

The crucial stage in this development was certainly the discovery of print. Just like the invention of writing, it initiated processes leading to a specific qualitative leap, both changing the way people use information gathered in culture, and introducing completely new kinds of messages (cf. McLuhan 1996). Although writing facilitated the transition from the "language of action" to the "language of reflection," the latter remained for centuries the domain of narrow elites having access to handwritten books and documents, because it was developed mainly for religious, scientific and administrational purposes. The invention of print not only contributed to the gradual spreading of this new type of language and the style of cognition associated with it (which was achieved mainly by extending the education system; cf. Marody 1987) but also gave the masses access to already accumulated information, while at the same time initiating processes in which those masses would introduce their own content and thus overcome the elite's monopoly in this domain.

The second type of transformations was related to the development of specialized tools meant to improve communication among people. For thousands of years since the emergence of language, direct physical contact was necessary to communicate with each other. Communication was thus constrained by the distance needed to be covered to establish such contact and also by all kinds of natural obstacles, often difficult or impossible to overcome, as well as the fear of Aliens, which inclined one to avoid contacts rather than seek them. The avalanche-like development of means of mass communication witnessed since the end of the nineteenth century - both ones allowing to cover huge distances and ones allowing to communicate directly at a distance - played a key role in the transformation of two fundamental concepts around which human thinking has been formed: time and space. For many researchers, this constitutes one of the fundamental characteristics of the newly emerging type of society (Harvey 1995; Castells 2001a). Moreover, it has also had a huge impact on the transformation of the third carrier of culture understood as accumulated information, i.e. human habits.

\subsubsection{Habits}

Habit is one of the oldest and thus least clear concepts in social sciences. The transformation of its meaning - from Aristotle, who considered customs to be our second nature, to behaviorism, which saw it as a trained reaction to stimuli, and to Bourdieu, in whose theory habit turns into habitus, or a socially 
constituted and legitimized scheme of dispositions to act and think - was summarized by Jean-Claude Kaufmann (2001). He also attempts to restore the original meaning to the concept of habit, viewing it as "a piece of social memory, individually embodied" (105). It is Kaufmann's understanding that is adopted in this discussion.

Habit is essentially non-reflexive: "habits gain force only when they disappear from conscious thought and become inscribed in hidden memory" (166). In other words, habit is the tendency to act in a specific way, somehow inscribed in the human body and actualized without the participation of our consciousness, or sometimes even against the conscious will. ${ }^{115}$ However, the "embodiment" of habits does not mean that they can be treated merely as a property of the biological body. After all, habits are formed in the process of acting, which involves objects and people, all of them being carriers of social memory. ${ }^{116}$ This runs counter to the assumption, commonly made in contemporary sociology, that all individuals create their worlds from scratch, using the power of their minds to discover the immanent properties of objects, and negotiating definitions of situations with other social actors. It would be more adequate to claim that people develop habits by "adapting" their hands to the properties of hammer (its very shape contains the social memory of specific actions), and by "adapting" to the behavior of other people, whose habits, in turn, contain memories of past interactions and social cooperation, sometimes even memories of solutions to various problems. ${ }^{117}$

115 In this sense, habits are more similar to unconditional reflexes rather than instrumentally conditioned reactions to stimuli which were studied by behaviorists (cf. e.g. Skinner 2002).

116 Dennett draws attention in this context to the kinds of changes in behavior that occur among elderly people who have changed their environment (e.g. as a result of moving to old people's home) and have been deprived of all their memory "anchors." He concludes that " $\mathrm{t}]$ aking them out of their homes is literally separating them from large parts of their minds - potentially just as devastating a development as undergoing brain surgery" (1996, 138-139).

117 A perfect illustration of the last issue is provided in an anecdote related by Linnda Caporael (1995), which tells of a young woman who would always cut a slice off the end of a piece of meat before placing it in the oven. Asked about the reason for doing that, she replied that this was something her mother would always do, who in turn imitated her own mother. It was only during an interview with the oldest of them that the reason was finally revealed: for many years, she used a roasting pan that was too small for the entire piece of meat, which meant she had to cut it. 
Insofar as most of our habitual actions involve use of tools and entail some form of communication with others, habits understood in the above sense appear as a fundamental carrier of the information that forms a part of human culture - one that includes a concise "record," not only of information stored in tools and language, but also of socially preserved ways of coping with fundamental existential problems and with the ties between people, which have been constructed on top of these problems. This is aptly demonstrated by Stefan Czarnowski in his 1956 essay, which attempts to explain the diffusion of technologies in Europe at the turn of the nineteenth and the twentieth centuries. He focuses on cases in which people rejected tools that were evidently more effective and allowed to achieve desired results more efficiently and with lesser effort on the part of individuals. His analysis clearly demonstrates that these tools affected both individual habits and their more complex structures. A hand "adapted" to a goose quill could not transform itself so as to efficiently handle a penholder with a nib; it was only the young, who had little or no practice in writing, that could "embody" the new tool by developing the kind of manual dexterity that was necessary to master the new habit. The spinning wheel made the thread even and allowed to spin faster, but nevertheless immobilized women, not letting them spin and simultaneously oversee work in the garden, or play with children, which the spindle made possible.

However, the most interesting example analyzed by Czarnowski is the resistance that manifested itself when the wooden plough was being replaced with the improved metal plough. This example shows that some technological innovations interfered not only with existing individual habits but also with the dominant pattern of ties within a group, in consequence endangering the stability of social relations. Using a wooden plough established a specific structure of dependence within the team of working people, a structure in which the husbandman played "a role similar to that of one steering a fishing boat: he worked with others, at the same time directing their work. While working, he was the same kind of ruler he was in his patriarchal country house among family and household members" (130). The new plough, as Czarnowski demonstrates, undermined the husbandman's special position, making him almost dispensable: even a woman could plough a field now, not to mention a farmhand or the farmer's son. Therefore, the introduction of a new kind of plough not only altered previously embodied habits, but also affected the structure of peasant family, which was vaguely sensed by Breton peasants who viewed the invention as a harbinger of moral decay and heralded "the rise of sons against fathers."

As is clear from these examples, habits are a factor stabilizing social actions and consequently - the relations between people, which arise from these 
actions. ${ }^{118}$ This does not mean, however, that these relations are permanent and unchanging. On the contrary, "their fundamental characteristic is dynamism" (Kaufmann 2001, 154). "Habits," Kaufmann writes further, "are not satisfied with repeating the past - they also record the new" (152). He emphasizes that the stabilizing effect of habits does not stem from the repeatability of certain actions, but from the continuous process which is the essence of collective life and in which individual habits are adapted to one another within the framework of a larger structure, where relations are formed in response to the habits of others and in contact with the material world. However, it means that, in order for this process of mutual adjustment to be ultimately successful, i.e. to stabilize social reality, it has to occur within a certain framework, or in accordance to specific rules whose breaking would deprive reality of its "common" character sustained by habits.

It could be argued that the said Breton peasants regarded the plough as a threat not just because it violated the structure of their habits, but primarily because it affected them so deeply that a certain fundamental part of common, habitual reality was shattered, undoing the image of the world in which sons, farmhands and women held specific positions established in relation to the husbandman. It is worth mentioning that in this perspective the said "image" is not a starting point for the formation of habits, but emerges somehow as a result of their disturbance. Action precedes thought or - as Kaufmann puts it - individual reflexivity occurs when various, often conflicting schemes of action come into contact, causing internal conflict and forcing the individual to reflect. This happens regardless of whether it would be a reflection on the gesture of the hand that, previously "trained" so as to use a goose quill, now loses its ease of movement when shifting to a penholder with a nib, making it more difficult to write letters, or whether it would be a reflection on the changing nature of the world, in which - as Czarnowski writes - "sons and farmhands began to get out of the husbandman's hand."

"People do not have habits, but are made of them; when it comes to regulating behavior, they are almost entirely made of habits," Kaufmann argues

118 Hence the process of developing habits is usually treated as the basis for their institutionalization. This is how Berger and Luckmann treat it: "Institutionalization occurs whenever there is a reciprocal typification of habitualized actions by types of actors" $(1991,72)$. However, they treat the term "habit" as an equivalent of "routine," assuming the repeatability of certain actions, i.e. performance in accordance with a certain pattern, which - as we shall see later - contradicts Kaufmann's conceptualization adopted in this book. 
(2001, 151), claiming that habits lie at the foundation of making reality common and banal - a process that "simultaneously creates the smoothness of gestures, the obvious points of reference and the 'simply here' effect of familiar setting" (123). Therefore, as long as surrounding reality remains in accordance with our habits, there is no necessity to ponder over its image. Thinking begins when a "gap" opens between our habits and those elements of the outside world that are or have to be engaged in carrying out these habits: tools, ideological or scientific messages promoting certain patterns of behavior, habits of other people, and nature.

Thus, as Kaufmann notes: "It is not individuals as such that shape their reflexivity in order to (possibly) change the course of action, but social contradictions, which are expressed in conflicts of schemas, prompting reflexivity and - with its mediation - facilitating subjective initiative" (155). Let us note, however, that insofar as those "social contradictions" are produced in contemporary society by the coexistence of various schemes of actions, interiorized by individuals in the process of socialization, and by the coexistence of competing "ideologies of life," whose summary is contained in the form of tools and messages that constitute the contents of culture, in earlier periods of human history, when "holistic" communities dominated, such contradictions were revealed mainly in contacts with representatives of other cultures, or in the course of action taken with regard to the natural environment. It is the move from the society in which individuals constituted a part of an integral whole, i.e. a world organized around socially selected habits, to the society in which the place of the Alien begins to be occupied by the Other, that can be treated as the fundamental historical threshold in the process of transforming habits understood as carriers of information gathered in culture.

This long-lasting shift from societies in which individual habits support and complement each other, upholding the socially constructed reality, to societies in which individuals are constantly exposed to the influence of competing habits that create diverse visions of the world, or emerge from such visions, is accompanied by two important processes. The first is a specific "acceleration" of reflexivity, related to the initially slow but now rapid rise in the number of the said "gaps" in individual habits, which need to be "covered" with thinking. Invoking a concept developed by Beck, Anthony Giddens observes that "[1]iving in the 'risk society' means living with a calculative attitude to the open possibilities of action, positive and negative, with which, as individuals and globally, we are confronted in a continuous way in our contemporary social existence" (2006, 28; emphasis added). What is more, the "radical increase in reflexivity" ceases to be limited to the thinking of "here and now," accompanying habits actualized at a given 
moment, but begins to cover the past, ceaselessly reinterpreted from the perspective of the present, and the future, or more specifically: "futures" that are "organized reflexively in the present in terms of the chronic flow of knowledge into the environments about which such knowledge was developed" (42).

One consequence of the "radical increase in reflexivity" is that habits lose their fundamental quality which derives from the "embodying" of fundamental operational schemes and manifests itself precisely in the smoothness and a-reflexive nature of so many actions taken by individuals. In contemporary societies, where the possibility to make choices has been raised to the rank of the chief principle ruling over all actions, it cannot be further claimed that " $[\mathrm{h}]$ abitualization carries with it the important psychological gain that choices are narrowed" (Berger \& Luckmann 1991, 71). The possibility and constant necessity to choose between competing operational schemes that assume the permanent presence of "imagined," alternative goals of actions, and to continuously adapt these actions to rapidly changing conditions and ever new people, who in turn enter into interactions by introducing their own habits, often radically different from ours - all this has triggered the second process, i.e. the emergence of specific meta-habits: rationality, planning, as well as being elastic, open, spontaneous or "cool."

The above meta-habits represent attempts to introduce orderliness and "normalcy" to the contemporary reality, whose chief feature is variability and fluidity, which stem from the rapid increase in the pace of technological changes, and from the growing "multiculturalism"119 of particular societies. In turn, however, they contribute to the intensification of the said feature, because accepting variability entails consent to or even encourages experimenting with operational schemes that are part of our habits, thus introducing an additional changeinducing mechanism to the surrounding reality. The source of ideas for these experiments does not lie anymore in the person's experiences gathered in the course of interacting with nature or other people, but can be found in the huge amounts of information amassed throughout the centuries in individual cultures and now "merged" through new communication technologies.

\subsubsection{Factors determining the transformations of culture}

This necessarily brief survey of the human mind's "peripheral devices" - tools that carry accumulated social memory inside themselves - and the indication

119 We put this term in quotation marks because in the light of theoretical assumptions adopted here it would be rather more appropriate to speak of a "differentiation of group representations within dominant forms of sociality." 
of the transformations that they have undergone in the course of history make it possible to distinguish three basic factors that underlie changes in human culture.

The first is rooted in the specific "openness" of all analyzed media. None of them has been developed once and for all. As a specific "peripheral device" of the human mind, each can be subjected to transformations whose direction is determined primarily by the consequences of its use. It could be said - summarizing some of the above points - that every one of them is "open" due to its very structure. This allows, in the case of language, for free recombination of words; in the case of tools - for experimentation with their usage; and finally, in the case of habits - for the addition of new links to existing chains of actions. Additionally, language is "opened" by the ambiguity of its messages, anchored in the intentions of the senders. ${ }^{120} \mathrm{~A}$ tool is "opened" by nature, which puts up resistance and enforces perfecting it. Finally, habit is "opened" by the counter-habits of other people, which make it visible and allow it to become the subject of reflexivity.

The second factor behind the transformations of human culture is a derivative of the interdependencies that link all the discussed media. This is especially clear in the above analysis of habits, which involve actions, use of tools, and communication with others. Any of these elements could trigger transformations of habits - different habits of another person, a new tool, or a new conceptual representation - and simultaneously entail adjustment of other elements. Generally speaking, a change in any of the carriers of culture affects others, which increases the probability of changing their users' ways of thinking.

This is closely related to the third factor. What we find at its foundation are specific qualitative leaps that occur in the process of historical changes in all the analyzed basic vehicles of culture. Each of those "leaps" has contributed to a dramatic change in the functioning of the human mind. Taking into account the fact that they did not occur simultaneously but sequentially, every further "leap" was a consequence of changes triggered by the previous one. The formation of the meta-tool that science has become today demanded the earlier development of a meta-language, while the emergence of meta-habits could be regarded as the effect of moving from the "language of action" to the "language of reflection," the latter combining strictly linguistic changes with scientific achievements.

Taking this sequence into account, one could say (risking gross simplification, a step that is nevertheless worth taking to ensure clarity of argumentation)

120 This is the origin of the necessity to negotiate meanings, which is postulated by interactionism. 
that the emergence of meta-language, meta-tools and meta-habits marks the beginnings of further stages in the development of human societies, or - to put it in terms closer to the idiom adopted in the this book - it accompanies the emergence of consecutive, qualitatively different forms of sociality, which correspond to the move from the traditional society to modern and postmodern one. However, three caveats are necessary here.

First, it needs to be remembered that although we speak about changes in thinking, which are a consequence of changes in the human mind's "peripheral devices," the processes described above are basically related to the transformation of culture as an information resource, not to the evolution of individual minds. Strictly speaking, one ought to say that the discussed changes translate onto the functioning of the human mind (and consequently - of human societies) only insofar as (and to the degree that) individuals and social groups "make use" of information contained in those basic carriers of culture. Given the unchanging nature of genetically programed properties of the brain ${ }^{121}$ - whose evolution has concluded in distant past - the mode in which the minds of contemporary people function becomes in this perspective linked to at least three other elements: "the technologies of the intellect," which allow to store and use information; socially differentiated processes of socialization, in the course of which individuals acquire different scopes of competences, allowing for more or less fluent use of the said "peripheral devices" of the mind ${ }^{122}$ (cf. Marody 1987); and the dominant forms of sociality, which act as a mechanism of selecting contents, both those processed by the mind and those that constitute its product.

Second, it means that a sense of progress, which accompanies the transformations discussed above (primarily regarding technological progress), does not stem from some fundamental changes of humankind as such, but from the accumulation of information that occurs in culture. This information is a

121 What is referred to here is the unchanging character of the species, which naturally allows for the existence of numerous variations on the individual level.

122 In this sense, we do not share the optimism expressed by Dennett, who claims that we "are the beneficiaries of literally thousands of such useful technologies, invented by others in the dim recesses of history or prehistory but transmitted via cultural highways, not via the genetic pathways of inheritance" $(1996,139)$. In our view, those "highways" are fitted with specific "toll gates" that grant access only to a relatively small part of the population (even today), whereas the rest is still moving along narrow, old "paths," the only difference being that the latter are socially made. Cf. a broader discussion of this problem in: Bourdieu 1986 (especially the part devoted to the conversion of one form of capital into another); Bourdieu \& Passeron 2000. 
product both of historical changes in particular carriers of culture, and of the individual creativity displayed by those who utilize cultural potential. As a result, it is not only the continuing but also the very maintaining of the achieved level of culture that demands from the population to keep recreating the competences that allowed to reach that level. This needs not only formal teaching, but also contacts with people who are "carriers" of these competences. ${ }^{123}$ At the same time, however, it is important to remember that the cultural "domestication of the savage mind," as Jack Goody termed it (1978), has not deprived us of all the elements of our "animal" equipment. Contrary to what has been assumed either explicitly or implicitly - culture has neither supplanted nor substituted biology in humanity's development; it is rather woven into it in a way that is unique to the individual and specific to a given form of society. ${ }^{124}$ Its impact is therefore dependent both on the repeated process of "embodying" habits, skills and beliefs in new-born individuals, and on the quality of those collectively sustained elements of cultural equipment.

Third, and finally, it turns out that we do not deal with a collective "March of Progress," but rather with a very long journey (to retain the metaphor) of a disorganized crowd. In this mass of people, some are marching forward, while others stand in place, sit down comfortably, go around in circles, or even turn back towards the point of departure. Everyone is constantly running into everyone else. To put it in less visual terms, at every point in time and space the ways of socializing human minds are radically different, not only in terms of content (this has been traditionally emphasized in reference to cultural diversity) but also in terms of the more general features of the "will" which lies at the foundation of individual action. In other words, in every particular, historical human

123 This fact has been indicated by Michael Polanyi (1967), who emphasizes that a large portion of our competences have the character of "tacit knowledge," which cannot be put into words but which can be acquired only through practice, preferably under the tutelage of an adult (in the case of children), or a "master" (in the case of adults). This constitutes the essence of socialization processes, both primary and secondary, which is discussed in greater detail in Chapter Two.

124 Cultural training basically boils down to the creation of neural links in the brain, i.e. filling the purely biological "organ of thinking" with social content. However, this "organ" is certainly not a tabula rasa, because it is fitted with older, evolutionary and instinctive action-controlling mechanisms (cf. Damasio 1995). Regulating those instinctual mechanisms constituted the fundamental function ascribed to culture in traditional views. In our approach, it constitutes merely one of many functions performed by culture in the process of "humanizing people." 
collectivity that forms a distinguishable whole, we can observe the coexistence of individuals who have interiorized different sets of skills stored in human culture (different both in terms of scope and structure). At the same time, the degree of that differentiation increases along with the rise in the complexity of information accumulated in human culture, the growing size of the human population, and the constantly improved access to means of communication that facilitate exchange of information. ${ }^{125}$

It was the abruptly rising degree of this differentiation, which accompanied the shift to the modern society, that foregrounded in classical works the question about the dominant principle coordinating collective action. This question would also lead to the distinguishing of different types of societies. However, before we turn to a more detailed analysis of these types and the mechanisms responsible for their emergence, let us briefly return to the concept of community and the social bonds that constitute community's foundation.

\subsection{Transformations of social bonds}

In combination with previous findings, the above survey of processes fundamental for the "development of thinking" - which, as Tönnies argues, have contributed to the emergence of the "arbitrary will" from the "natural" one, and then

125 This differentiation could be analyzed in many dimensions. However, taking into account the problems addressed here, the most promising path would be to adopt the differentiation introduced in the previous chapter, namely one between socialization as the acquiring of "technical" skills allowing for functioning effectively in a given society, and socialization as an emotional bond with the collective "We." Assuming those two modes of socialization as properties that individuals have either acquired or not, we are provided with a fourfold classification (introduction of an even minimal gradation would lead to its further expansion). Let us draw attention to the fact that sociology usually mixes those two types of socialization and exhibits a tendency to address mainly the two extreme poles of this classification, referring either to individuals socialized in the highest degree in both dimensions ("model" members of a given society), or those who are poorly socialized (representing the so-called margins). It is also worth mentioning that all analyzes of interactions would demand, within this approach, to account for three other typologically differentiated forms of sociality, which manifest in the differences in social organization. In particular cases, we might deal with contacts in which one individual is, for example, socialized in the "technical" dimension at the level of modern society and in the "social" dimension at the level of traditional society, while the other has technical skills at the level of postmodern society but remains entirely unsocialized in the dimension of the collective "We." 
to the rise of the "reflexive" will analyzed by contemporary sociologists - leads to two more general conclusions worth explicating here.

First, the analysis of the circumstances in which the above "qualitative leaps" occurred in the evolution of particular carriers of culture, even if brief, clearly demonstrates that they had to be preceded by a radical change in social conditions. Writing existed in many forms long before it gave rise to the "language of reflection" in a specific socio-politico-economic regime of Ancient Greece. It took even more time to make this new type of language widespread. Science separated itself from religion already in the Middle Ages, but it was only at the onset of modernity that this led to the development of what could be called the modern scientific approach, while really ground-breaking advances in this domain were made even later. The beginnings of processes that Norbert Elias termed the "psychologization" of thought and behavior have their roots - as he demonstrates (2000, especially Chapter Four) - in the court culture of the late Middle Ages, but it was only in the twentieth century that they reached the stage we now call "reflexive."

Obviously, one could explain these processes - as is often done - by referring to the innovativeness of individuals and the gradual dissemination of their "inventions." However, as has been already pointed out, the "physical" emergence of particular innovations in itself did not suffice to make them influence the way people think. This is confirmed not only by the case of those original tribes that have survived to this day, and whose "hindered development" could be explained by their being cut away from communication with the mainstream of social changes around the world, but also by cases of such societies as the Chinese one, which slowed its development at a relatively early stage in history, or by cases of modernization failures suffered in the twentieth century, for example in African countries. Therefore, it is necessary to assume that, in order to change ways of thinking at a larger scale (population-wide), it is vital to transform not only the "peripheral devices" of the human mind but also the dominant forms of socializing processes. The latter - as we have shown in the previous chapter - constitute the basic mechanism of selecting outside influences, regardless of whether it is the natural environment or the sum of all products made with human hands and minds.

Second, if transformations of ways of thinking are - at least to a certain extent a derivative of changes in dominant forms of sociality, it means that communal bonds have a unique status in comparison with two other types of relations and collectivities created by them (see fig. 3.2a.). As we demonstrate in the previous chapter, the basic factor explaining the phenomenon of sociality is the emotional bonding of people, which lies at the foundation of the sense of community and 
resultant obligations to this particular social entity. Therefore, regardless of what form this sociality takes - be it traditional, modern, postmodern, or of any other, local type - its constitution must rely on some form of communality. In other words, the claim made by Tönnies, i.e. that arbitrary will develops from natural will, has to be given a much stronger sense than the one originally intended, both in terms of its temporal sequence and the "genetic" conditioning. The kind of attitude towards reality that corresponds to historically given manifestations of the arbitrary will would be dependent on historically given characteristics of the natural will, just like the properties of particular forms of "association" would depend on the properties of communities preceding them.

It can be easily observed that this conclusion alters the relationships between types of wills and types of communities, both those assumed by Tönnies, and those included in fig. 3.2a. which seeks to extend Tönnies' typology so as to cover the current period. This conclusion implies that although changes in human thinking triggered by changes in basic carriers of culture influenced the constitution of more permanent bonds between people, there is no simple transition between the "development of thinking" and changes in the types of wills and in accompanying forms of collectivity. If so, it means that fig. 3.2a. mixes two orders: first, the historical one, which accounts for changes in forms of sociality and accompanying changes in ways of thinking (transition from societies in which communal bonds are dominant to societies in which network relations prevail; the transition from cultures of shame to cultures of authenticity); and second, the typological one, which accounts for the different forms of relations between people and the resulting different types of social relations. ${ }^{126}$ The separation of these two threads could begin with the typological order, to which we now turn.

In light of the above considerations of community, its most characteristic feature seems to be the sense of unity. At the same time, however, we need to remember that this unity does not stem from everyone being identical, or taking identical actions; it rather has its roots in the fact that all members of community feel linked to something that exists above them, something that might not endure if it had not been for the constant co-presence and co-operation of its members. In this sense, a marching military unit is not a community, despite the fact that all soldiers act exactly the same way, whereas a family whose members focus on different tasks is a community or could be one under the condition that these tasks are subordinated to the supporting of the family's existence. One

126 As mentioned above (cf. footnote 12), mixing of these two issues can be also found in Tönnies' theory. 
could therefore say that bonds created within a community are characterized by a specific form of interdependence among individuals, rooted in the complementary character of their actions with regard to the higher "goal:" to support the community's existence. These actions are not so much reflexive as primarily habitual, i.e. derived from the mutual "adapting" of people to each other within relations and in contacts with the environment, which creates customs and tradition. The complementary actions are accompanied by common preferences (sentiments) ${ }^{127}$ and shared "knowledge" stemming from memories of living together. It is thanks to this and to the subordination of one's actions to the good of the community that its members form a whole.

The complementary character of actions means that individuals create a whole of which they are part, and which "acts" jointly towards a unified goal. Let us emphasize here that such a complementarity simultaneously entails the diminishing of individual freedom (the autonomy of individuals with regard to the community), of equality (there is no ground for comparing individuals if each of them differently contributes to the life of the community), and of individual independence. It means that complementarity makes it difficult to preserve the principle of justice, i.e. ensure equal contribution to actions (costs) and equal participation in effects (benefits). Thus, complementarity is basically incommensurable at the level of its components.

Freedom, equality, independence and justice can emerge in cases of social relations constituted on the grounds of exchange - at least inasmuch as there do exist external, objective measures of value (e.g. in the shape of a market that sets the prices for wares). ${ }^{128}$ Certainly, such ties, especially when given a more durable character, also establish a certain interdependence and necessitate mutual "adapting" among the people who establish them, but this process has a different mode and character. When selecting a gift for someone, we may try to "adapt" its value to that of gifts received from that person in the past, but this kind of interdependence, which occurs at the level of exchange, covers only a tiny aspect of our interactions and could even be connected with a complete lack

127 This does not mean, however, that individuals need to love each other or share any other stronger feelings. What is rather at stake here is the similarity of attitudes towards outside events or objects; hence, these are shared rather than reciprocated feelings.

128 The lack of such measures might be one of the causes of problems in marriages based on "partnership," where a "fair" division of duties is achieved by reducing the relationship to the level of an exchange of "services" between spouses. We shall return to this issue later. 

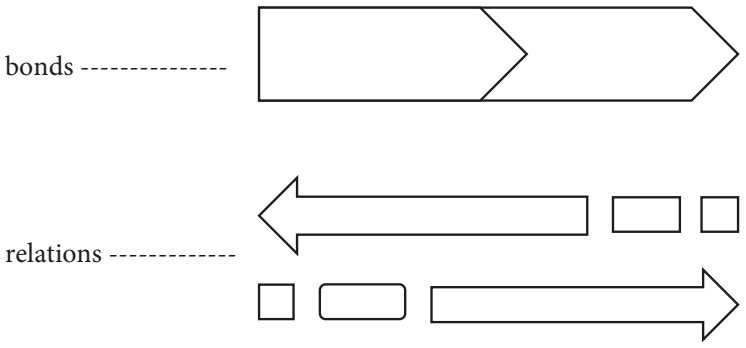

influences
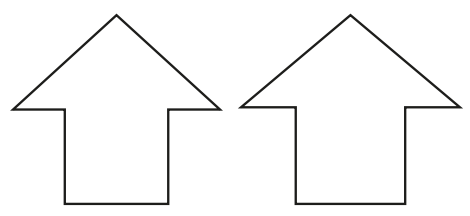

similarity

Fig. 3.2b: Types of social interdependencies

of adaptation if, for example, the basis for action - its "goal" - is the subjective compulsion to reciprocate.

To put it differently, the kind of interdependence that is established at the level of exchange "binds" individuals much more weakly, not only because it has an aspectual character but also, or even primarily, because it demands reflection and necessitates forming on each occasion a subjective "goal," which the reciprocation must serve. ${ }^{129}$ In turn, achieving real complementarity demands switching off reflection and is related to taking-for-granted the "goal" that stands before members of the community.

To avoid misunderstanding, the concept of "bonds" is reserved here for ties based on complementarity, while ties established at the level of exchange shall be described as "relations." As can be easily noticed, these two types of interdependence correspond to Tönnies' division into community and society. However, since the question about the ways of coordinating human actions could be considered as crucial for his typology, we should point out here that there exists yet another type of interdependence among individuals, namely one based on similarity (cf. fig. 3.2b.).

129 As Bourdieu has shown (1986), giving presents or exchanging favors is a real art that plays an important role in building social capital. 
Coordination of actions does not have to be based on subjectively identified relations between individuals, although it does not preclude the possibility of their mutually influencing each other, or their actions "harmonizing" due to their "tuning," achieved on the ground of similarity in tendencies to act. We deal with this kind of coordination for example during social events gathering people who do not know each other, at public demonstrations that attract occasional passers-by, or in situations when a crowd chooses to behave in some particular way. ${ }^{130}$ Interdependencies created as a result of such actions usually have an ephemeral character, being limited to joint participation in a specific event. Even so, they may strongly affect individuals: in certain cases, they can become a springboard for establishing more permanent relations or even forming bonds. The latter usually involve a significant share of the emotional component, whereas the former - lack of broader obligations with regard to other participants.

Let us draw attention here to the specifically inclusive nature of the three distinguished types of social interdependence: bonds include both relations and influences found among individuals, whereas relations do not have to lead to formation of bonds but certainly cover influences, with the latter constituting an irreducible basis for the most rudimentary type of relationships. ${ }^{131}$ Therefore, one might agree with Tönnies when he argues that there are no collectivities deprived of elements of both Gemeinschaft and Gesellschaft, if we reduce those two terms to relationships based on complementarity and exchange. However, it is something completely different to claim that community-like collectivities (constituted

130 It seems that Simmel's "sociability" could be included in this category of relations (cf. Simmel 1950).

131 The dissimilarity of the three kinds of relations has been noticed in social sciences a long time ago, just like the dissimilarity of the influences they exert on individual actions. This is best visible in Merton's theory of reference groups (1968). This theory usually distinguishes, first, normative reference groups, with which the individual has a strong emotional bond, and which rely on the acceptance of group norms and values; and second, comparative reference groups, established by individuals on the basis of more objective relations linking them to certain categories of people. Maria Łoś supplements this typology with the "audience reference group" (1976). The existence of such a group could be recognized by individuals despite a complete lack of any stronger bonds or relations linking them with people forming it and the subjectively perceived existence of such a group may affect individual behavior, which corresponds - in the classification adopted here - to relationships based on influence. Nevertheless, the theory of reference groups implicitly suggests that differences between groups correspond to differences in relationships, which contradicts the principle of inclusiveness adopted here. 
through bonds) can include in themselves collectivities of the Gesellschaft type (constituted only through relations of exchange). It would entirely contradict both assumptions made within Tönnies' typology, and available data showing tribal communities as collectives that have not developed specialized subgroups organized around relations of exchange.

It rather ought to be assumed that the creation of distinct collectivities based solely on relations or influences is part of the historical order. That is why we should consider the process of their emergence in separation from the typological order. At the same time, however, it also ought to be assumed that this process did not entail the transition from communities to societies, or other forms of collective life, but rather relied on the production of new kinds of social groups, whose creation was accompanied not just by the disappearance of communities, but rather by in-depth transformation of communality as a specific social bond determining the framework of sociality.

It is not our aim to investigate the causes of this historical process here they are analyzed in detail in subsequent chapters. However, let us draw attention to the fact that this process must have been combined with the intensification of contacts among individuals belonging to different communities, who were for that reason not tied with social bonds. It was only due to such contacts that "arbitrary will" could develop - the kind of thinking that we find at the foundation of societies, and thanks to which individual actions cease to be based on traditional habits and begin to be the subject of reasoning and goal-oriented decisions. On the other hand, it needs to be remembered that a sense of community - subjected to transformations - must have retained at least some features of Gemeinschaft, which is the model example of a community based on bonds. In other words, new forms of communality had to be still created through the natural will, resulting in the creation of such kinds of social relationships that allow individuals not only to feel related through a sense of belonging but also attached through faithfulness, thankfulness and loyalty, which transform individual emotions into moral sentiments, consequently turning obligations to some group into something natural, something that is not given special consideration or reflected upon. It is thanks to this that basically all members of the community carry the entire collective inside themselves (Gauchet 2000,33). This also allows us to speak of socialization of human actions.

If we adopt this perspective to consider the consequences suffered by social bonds as a result of transformations induced by the historical process transforming social relations and dominant ways of thinking, it could be generally said that the direction of these changes is most often defined in terms of the 
"cooling" or "dissolving" of this type of relationships between people. However, this statement demands further explanation.

The tribal community formed in the process of "foundational holism" combined two functions: it was a unit of both biological and social reproduction, or to put it differently - it was both a group of families, and a total, socialized unit of adaptation to its surroundings. Although it is possible to differentiate between the two dimensions analytically, in reality they were tightly interwoven, while connections created in the course of being-shared-with-others would constitute a collectivity in which blood ties overlap with social bonds.

The emergence of new types of relationships between people, which occurred in the process of historical change, entailed the creation of additional social groupings between the level of family and that of the all-encompassing social system. On the one hand, this weakened relationships within the family, the basic group of origin; on the other, however, it imposed the necessity to incorporate such groupings into the overarching community. After all, if the entire social system were to retain its adaptive character, it had to support its ability to integrate all actions: not only those of particular individuals but also those of numerous and often antagonistic social groups created by those individuals; moreover, it had to be capable of generating actions meant to support its existence. In other words, it had to have the potential to create bonds, i.e. to emotionally bind people at a systemic level. ${ }^{132}$

Norbert Elias seems to have had this kind of processes in mind when he introduced the concept of "units of survival" (2001), enumerating the tribe, the state and humanity as basic systems of social integration, which have developed subsequently in the course of historical transformations. Adopting such a view of changes discernible in human history makes it possible to better understand the above claim regarding the "cooling" of social bonds. Although in the case of tribes, there would be no real difference between individual socialization, occurring within a family, and the socializing processes taking place on a

132 As Stanisław Ossowski demonstrates in his excellent article "Więź społeczna i dziedzictwo krwi" ["Social bond and blood heritage"] (1966), one of the ways to reach this goal was to support a belief in shared ancestors. A belief in the "community of blood," as well as the resulting strength of bonds and the permanence of obligations to community, are something that has not only survived to this day in the form of genealogical and ethnic (racial) myths but has also been transferred onto various "artificial" groups that are not based on common ancestors. In order to establish a "spiritual" community they often employ symbolic means and rituals, which offer a semblance of "blood ties." 
systemic level, social bonds created within systems based on the state undergo a kind of "diffusion" that disperses them among a plethora of various social groups before they conclude their passage from the level of family to the state-based level of integration.

It is worthwhile to draw attention here to the fact that it is only in the case of the first two "units of survival" that we can be sure about their creating distinct forms of social bonds. In the case of tribal communities, these were (and are) bonds based on common ancestors, while in the case of the state - bonds based on the concept of nation. Furthermore, it is only in the case of these two units that we can speak of the emergence of clear forms of social control, although instead of the above-mentioned division into cultures of shame and cultures of guilt (cf. fig. 3.2a.), a more sociologically adequate one seems to have been offered many years ago by David Riesman (1969), who has distinguished societies guided by tradition and "inner-directed" societies. ${ }^{133}$ Were we to assume, following Elias, that today's basic unit of survival is humanity - a frame of reference that fits perfectly both with findings about globalization processes and with Beck's most famous claim about the globalization of risk (1992) - there arises the question as to what might give rise to a sense of community on that level and what forms of social control it could be based on. We shall return to this question in the last chapter.

Historical changes of basic units of survival are one of the two factors behind the process causing the "cooling" of social bonds. The second factor involves the above-mentioned transformations of human psychic apparatus resulting from the evolution of "the mind's peripheral devices," which, in turn, has influenced and shaped ways of thinking. The transition from natural to arbitrary and finally to the reflexive will entails not only the intensification of reflection on one's actions but also, increasingly common, an analysis of the "natural" bonds that give rise to the community - ones essentially rooted in emotions and faith, not in reflection. They begin to be assessed in terms proper for society, i.e. with regard to such notions as justice, equality and independence - things that members

133 This exchange may seem, at first glance, a purely formal move, because in the description of both kinds of societies Riesman refers to the distinction between "fear of shame" and a "feeling of guilt" as the main factors regulating the behavior of individuals. However, his analysis of mechanisms of social control proper to the two types of societies goes beyond an individual perspective, not to mention the fact that the typology he proposes also covers the current period. Riesman's concept of "other-directed" society seems to have greater heuristic utility than the aforementioned concept of "culture of authenticity." 
of society have a right to. The ideology promoting "loyalty and faithfulness" which assumes that obligations to the community originate in one's "innate" feelings for the family and nation, or in other kinds of social relationships based on bonds - is supplanted by the ideology of "authenticity and pure relations," which accentuates primarily obligations of individuals to themselves, or to their own development.

Such dissolving of old, and the developing of new forms of socializing human actions, is a process shaped entirely by objective factors. However, in order to elaborate on them further it becomes necessary to examine in greater detail processes found at the base of all social changes. First of all, it is paramount to establish the way in which we can understand the social system as a unit of reproduction and adaptation to its surroundings. 



\title{
CHAPTER FOUR. SOCIETY AS A PROCESS
}

\author{
5,000,000,000 individuals in this world. Human society, \\ humanity, is, of course, nothing other than the totality of \\ these individuals. But these five billion individuals are \\ not running about singly or in loose groups, as they are \\ depicted in some older sociological theories, including \\ Max Weber's theory of action. Practically all these people \\ are organized in more or less fixed associations.
}

NORBERT ELIAS, Society of Individuals

The fundamental process takes place in the model of interpretation.

JEAN-CLAUDE KAUFMANN, Ego: pour une sociologie de l'individu

At the foundation of the paradigms that have gained prevalence in contemporary sociology, we find lonely individuals and their actions. Although they may be equipped with different scopes of agency, and their actions may be sociallyoriented to a varying degree, this does not change the fact that the starting point for most considerations of society is usually the isolated atom. Taken together, these atoms would comprise society, which emerges - by way of largely undefined processes - from "adding up" individual actions. The universality of this approach introduces into sociological theories - from the very onset - an abstraction that does not take into consideration the most fundamental quality of human existence.

After all, the fundamental experience of humanity is that of living in a group. From the moment of birth, our "natural" environment is constituted by other people, connected in specific ways through family ties and peer relations, and forming diverse social categories that comprise various kinds of societies. The sense of belonging to many, more or less permanent groups and collectivities is not only an addition to human social existence but its constitutive factor. Each person's individuality is shaped by his or her relations with other people categorized in terms of group membership, while the differentiation into "us" and "them" is one that establishes the world of both social and individual life.

This was emphasized already in the 1920 s by George $\mathrm{H}$. Mead, who argues that "the behavior of an individual can be understood only in terms of the behavior of 
the whole social group of which he is a member" $(1972,6)$. He explicitly claims that "[w]e are not, in social psychology, building up the behavior of the social group in terms of the behavior of the separate individuals composing it; rather, we are starting out with a given social whole of complex group activity, into which we analyze (as elements) the behavior of each of the separate individuals composing it. We attempt, that is, to explain the conduct of the individual in terms of the organized conduct of the social group, rather than to account for the organized conduct of the social group in terms of the conduct of the separate individuals belonging to it" (7).

Mead's followers have entirely disregarded this assumption. ${ }^{134}$ Instead, they have extracted the concept of interaction from the context of interdependences created for individual actions by the "organized conduct of social group." This move was certainly favored by the fact that the basic point of reference for interactionists in their attack on mainstream sociology was the normative paradigm derived from works by Parsons who - following Weber ${ }^{135}$ - constructed his theory on the basis of the category of social action, building the concept of social structures on top of it. In the second half of the twentieth century the focus on individual was favored by interest taken in "human agency"136 - increasing since the 1960s - which undoubtedly constituted a reaction to general changes in culture. Regardless of the reasons, however, maintaining this perspective not only introduced a false premise into the heart of all more general theoretical considerations but also made it more difficult to grasp the final "product" of

134 One of the few examples of attempts at developing this theme - albeit not a very successful one from our perspective - is Tamotsu Shibutani's Social Processes (1986), in which he tries to sketch a project of an interactionist sociology understood as a discipline that focuses on studying social processes. He defines the social process as "a pattern of joint activity that occurs regularly over time" (25). In practice, however, this boils down to analyzing processes by focusing on features characterizing the types of interaction that lay at the foundation of these processes.

135 As Norbert Elias (1978) suggests, it is precisely because Weber tries to draw a boundary between the individual and the social that he gives crucial importance to the concept of social action.

136 One attempt at synthesizing various concepts of individual actions from this perspective is found in an article by Mustafa Emirbayer and Ann Mische (1998). Their theory is characterized by something quite typical of most sociological theories that work on the micro level: it considers interactions as individual events or - at best - as classes of events, i.e. meetings of atomized individuals, which find no continuation in space and time. 
individual actions, understood in sociology as society, especially in the case of contemporary society.

Defined in systemic terms, society is usually viewed as a complex system described in terms of properties that are irreducible to characteristics of individual people. It is significant that such considerations of society often cause the concept of the individual to disappear from analyzes. Instead, scholars usually turn to the concept of social order described as a set of rules and institutions that would determine the framework of social life. In theories that focus on society as a social organization, individuals appear only as vehicles of social roles, officials at institutions, or elements of broader structural categories like classes. One could even say that this perspective does not leave any room to account for individual actions, which become - at best - an epiphenomenon of systemic rules.

In everyday practices of sociological research, the disparity or even sharp contrast between the two perspectives is not particularly painful, mostly because contemporary sociology has become increasingly specialized and multiparadigmatic. At best, one could argue that such coexistence leads to a specific "ontological dualism" (Poleszczuk 2002), in which the individual would be opposed to society. This also leads to the reification of society, which is ultimately perceived as an entity composed of structures external to individuals. As Norbert Elias vividly puts it, "the individual is at one and the same time surrounded by society yet cut off from it by some invisible barrier" $(1978,15)$. Sociologists are thus forced to cross that barrier every time they move from analyzes on the micro scale to ones on the macro scale.

The problem of relations between systemic properties and individual actions cannot be ignored while attempting to construct comprehensive systems that would provide a broader theoretical framework for sociology as a specific discipline within social sciences. At the same time, this is a problem that - given such extremely defined "boundary conditions" of sociological enquiry - cannot be solved satisfactorily without employing certain concepts that mediate between phenomena occurring on the macro level and those on the micro level. ${ }^{137}$ Actions of individuals are too particular and diverse to derive from them any systemic properties without taking additional steps. These properties, in turn, are too

137 We have drawn attention to this in an earlier work, demonstrating that the question of relations between both levels of sociological research is usually left unresolved, or even eliminated, by assuming an isomorphic character of relations between the level of systemic properties and that of individual actions. Cf. Marody 1991. 
general to allow drawing direct conclusions about characteristics of individuals and courses of actions they take.

It seems that two such "mediating concepts" enjoy the greatest popularity in contemporary sociology: institutionalization and emergence. Both concepts assume that individual actions take precedence over systemic rules. Moreover, in both cases these rules are considered as derivative of two processes occurring within a given society: first, routinization, and second, standardization of actions through either consolidation of certain habits, or the emergence of certain more general patterns of behavior (cf. Berger \& Luckmann 1991; Giddens 1986). ${ }^{138}$ Finally, at the foundation of both concepts we find the emergence of some innovations at the level of individual actions. These novelties are then spread by way of imitation, allowing to describe processes of social change.

However, such explanations tend to disregard one well-described fact that is particularly emphasized in analyzes of other processes: ${ }^{139}$ innovations do not spread randomly. Moreover, the ones that affect the level of systemic properties are bound to encounter resistance from those social groups that would prefer to preserve the system's previous shape. ${ }^{140}$ Thus, the process of institutionalization - through which systemic properties are supposed to emerge - turns out to be a process whose crucial component remains undefined. Consequently, the problem of relations between the level of individual actions and that of systemic properties remains unexplained. ${ }^{141}$

138 We disregard here the fact that various attempts at formulating a general account of the two concepts usually employ circular definitions (ignotum per ignotum). For example, the textbook by Sztompka defines institutionalization as "the emergence, articulation and consolidation of normative structures, social rules, as well as patterns, norms and values" (2002, 432; emphasis added). However, the concept of "emergence" tends to be unexplained and based on popular intuitions. If it were to be made more precise, the concept would probably have to be defined using the term "institutionalization."

139 Cf. for example Robert K. Merton's analysis of deviant behavior in Social Theory and Social Structure (1968).

140 Certainly, at least some of the problems arising from the concept of institutionalization are related to the use of a language that obliterates the difference between - for example - "the invention of a fork" and "the introduction of a new legal definition of private property." At a certain level of universality, the process of spreading each such invention could be described in the same way (cf. Sztompka 2002, 417-433), despite the fact that their consequences are radically different.

141 Of course, one may get around this restriction by assuming that the emergence of innovation is not accidental, but harmonizes with the logic of social development, which nevertheless leads to some kind of teleology and therefore involves many hidden dangers. 
In fact, it cannot be explained because the problem is simply artificial. It does not stem from the nature of examined "objects" but it is rather the effect of adopting a particular research perspective. In other words, the necessity to explain relations between the level of individual actions and that of society's characteristics is but a consequence of their previous dissociation by those theorists who, in their attempts to grasp the individual "atom" of social phenomena, have isolated individual actions from their societal context. After all, one cannot explain most individual actions without referring to the concept of society, just like the functioning of society cannot be explained without referring to individual actions. This means that the first step in any theoretical conceptualization of the two entities should involve - right from the start - restoring their mutual dependence.

\subsection{Levels of analysis}

The difficulties that arise while attempting to restore the interdependence between individual actions and societal characteristics stem primarily from the lack of a language suitable for capturing this interdependence. Consequences of contemporary sociology's "ontological dualism" include not only a shortage of terms referring to relations between the systemic level and that of individual actions, or a division of the discipline into macro sociology and micro sociology, but also the fact that each of those perspectives develops its own conceptual schemes that cannot be translated from one domain to another. ${ }^{142}$ Even more serious consequences are related to the belief in the "sovereignty and ontic

142 Attention to this was drawn already by C. Wright Mills, who emphasized in his criticism of Parsons' theory that sociology overestimates "common values" as the binding material of social organization, which would be accompanied by underestimation of power and various forms of violence - concepts tied to the systemic perspective he himself represented. "Even in such sacred little groups as families," he writes, "the unity of 'common values' is by no means necessary; distrust and hatred may be the very stuff needed to hold a loving family together." However, it seems that he has fallen victim of a similar (though reversed) bias that distorted his perspective, when he wrote that "[a] society as well may flourish quite adequately without such a 'normative structure' as grand theorists believe to be universal" (2000, 40). As Crozier and Friedberg have demonstrated (1980), even the most rational forms of social organization - understood in the narrow, literal sense - succumb to changes, mostly adverse, under the pressure of interactions established by their members. This would mean that the "normative structure," even if not universal, can be nevertheless important in explaining processes that occur at the macro level. 
precedence of the subject," which we have discussed in the previous chapter and which is increasingly shared by leading sociologists. Regardless of its possibly self-contradictory character, this belief conveys some hidden assumptions that cannot be reconciled with attempts to develop a systemic account of society. ${ }^{143}$ Treating it as the outcome of interactions between independent, autonomous actors means - as Linnda Caporael observes - that human "sociality" becomes a by-product of individualism. In this approach, what motivates human actions is a broadly understood self-interest since "'social' refers to the exchange of costs and benefits in the pursuit of outcomes of purely personal value, and 'society' is the aggregate of individuals in pursuit of their respective self-interests" $\left(1995,1^{144}\right)$.

Meanwhile, as we have attempted to demonstrate in the previous chapter, and as Caporael emphasizes, there is enough evidence that "humans are obligately interdependent, not only for acquiring their daily bread, but also for the daily operation of their minds" (2). The founders of modern social sciences considered it so obvious a fact that they hardly devoted any attention to it. As Caporael observes, "when Adam Smith proposed the 'invisible hand' of self-interest, he took for granted that the butcher, the brewer, the baker - and their families who worked in the business - were organized in workshops, which were in guilds, which were in villages, which were in districts, which had seasonal fairs and religious celebrations. In the eighteenth century, the skills for butchering, brewing, and baking were accumulated through generations, passed from adult to child, and repeated in daily, weekly and seasonal cycles of activity. Butchering, brewing and baking demanded finely tuned sensory and motor coordination; familiarity with variable materials, tools and methods; a marketplace, of course; and coordination among these physical, mental and social components" (2). This

143 As Zygmunt Bauman convincingly argues (1995), the concept of freedom cannot be articulated differently than by indicating the existence of such forms of dependence that are imposed by particular groups, and from which individuals set themselves free. In consequence, a subject cannot be "ontically precedent" to the group and simultaneously "sovereign," i.e. freeing him- or herself from that group's influence. This would be possible only when we assume the existence of a process that would (somewhat secondarily) subject the individual to the influence of his or her own "product" - the social group. This kind of assumption seems to be made by Anthony Giddens in his theory of dual structures (1986); however, he does not explain why "ontically precedent subjects" cannot control the products of their actions.

144 In references to the article by Linnda Caporael, which was published in an online magazine, numbers in parentheses refer to paragraphs, not pages. 
mechanism of reproducing social life was part of common knowledge among thinkers working in that period. As a result, Smith could allow himself to ignore it in his discussion of the market.

The fact that most elements of this mechanism look different today does not mean that people ceased to depend on each other, or that they act on the market or in other spheres of social life as fully independent and autonomous individuals. The popularity of such a conviction in contemporary social sciences demonstrates, at best, the failure to acknowledge the difference between simplifying assumptions made by past thinkers in their models of social reality, and assumptions regarding the very nature of that reality. However, as a result of the above neglect, and given the continuous processes of social change, something that did not have to be made explicit due to its self-evident character in the past, cannot be articulated today - when that reality changed its shape (though not its nature) - due to the lack of a suitable language.

Offering a very interesting, comprehensive approach to the analysis of social phenomena, Linnda Caporael tries to develop the kind of language which would make it possible to analyze the interdependencies that bind people together in social life while setting aside the question of the "ontological precedence" of the individual or society. The model proposed by her rests upon two assumptions that are fundamentally different from those traditionally held in contemporary sociology.

Caporael's first assumption about social life is that it ought to be regarded in terms of "nested hierarchical organization" comprising various levels of functioning joined in such a way that the (structurally speaking) "lower" entities of organization are "roots," or a kind of a "nest," from which "higher" entities grow, creating a basic "environment" of action for the former. Therefore, the functioning of a lower-order entity is fundamentally dependent on its functioning in the entire system, which constitutes the "interface" with the habitat. At the same time, changes at the lowest level of hierarchical organization (in this book - the level of individual actions) are incapable of directly affecting the entire system; in order to influence it in any way, these changes have to be first assimilated by higher-order entities. ${ }^{145}$

145 One example of such a "nested hierarchical organization" in the area of biology is the human organism. At the lowest level, its shape and functioning is dependent on genes that determine the growth of cells, which are the building blocks of specialized tissues, which compose individual organs (e.g. stomach), which comprise systems (e.g. the digestive system), which finally form the entire organism. The natural environment - i.e. the habitat - directly affects only the level of the entire organism; a gene, 
According to the second assumption, the organizing entities from various levels exist not as permanent beings characterized by fixed properties, but are ceaselessly reproduced in accordance with the temporal scale and rhythm appropriate for a given level. At the base of this process of reproducing lay certain specific relational linkages connecting a given organizational entity to its direct "environment" - the higher-order entity. Such linkages should not be considered - as in most sociological theories - to be a kind of a matrix that facilitates automatic transferring of features of society as a whole, or its constituent parts (classes, institutions, organizations etc.), to the level of individual actions. Rather, the nature of these linkages resembles a chemical reaction, which is possible only when all ingredients "meet" in a strictly defined context (environment). That is why relational linkages are a specifically understood principle of recreating a certain effect (the result of actions) in a certain environment.

Such an understanding of relational linkages lies at the foundation of Caporael's concept of "repeated assembly" - the basic unit in analyzes of processes of social life. ${ }^{146}$ She defines it as "recurrent entity-environment relations composed of hierarchically organized, heterogeneous components having

for example, uses the cell as the "interface" between itself and the habitat. The cell is also the gene's basic "environment" of action. Therefore, the influence of the habitat on genes is mediated by relations between all those levels of organization that separate the highest level (the organism) and the level of the cell. Conversely, damage to a gene could influence the entire organism only by affecting cells, which in turn affect tissues, and so on. This example is not meant to serve as a preliminary to constructing analogies between human organism and society, but rather aims to help in grasping the rather abstract terms of the assumption discussed here.

146 More precisely, it would be the basic unit for analyzing processes of life not only in its social dimension but also in the biological one. Caporael developed her model by taking cue from findings in biology and evolutionary theory. Throughout her argumentation she refers to those concepts that - in her view - simplify or even distort the truth by considering properties of human beings or their actions as a direct effect of genes. As she writes, "[w]e are no longer talking in the billiard ball universe of linear causes where we can imagine that genes cause or predispose traits, which are then modified by the environment. The analogy is closer to a shared language (relation) where (entity) speakers' utterances are both caused by and cause other (environment) speakers' utterances" (10). Finally, she derives the term "nested hierarchical organization" from those contemporary strands in evolutionary theory that attempt to restore Darwin's original understanding of the evolutionary process as occurring at the level of the entire species, not that of individual representatives. Cf. also on this subject Kaufmann 2001. 
differing frequencies and scales of replication" (11). For example, if we take language to be a kind of "repeated assembly," we could say that for the linguistic competence to emerge on the individual level it is necessary, in each case, to establish a relational linkage between particular, genetically coded elements of the individual organism, which facilitate acquisition of language, and their exposure to a linguistic environment, which constitutes a critical component in the process of "assembling" those elements.

Phrased in this way, this account could seem - at first glance - to be a merely awkward reiteration of a broadly accepted claim that in order to learn a language it is necessary to be stimulated by the social environment. However, as Caporael demonstrates, the discussed definition assumes something that has been completely neglected in traditional approaches, namely the reproduction of not only human genotype but also the social environment. Thus, "both the genes and the language environment must be inherited. English speaking and Kikuyu speaking differ because the various elements have different cycles of repetition. One set, which includes genes, has a longer cycle of repetition relative to another set, which includes the language environment. Both sets are repeatedly assembled, but on different scales of time. The cycle of language environment (English or Kikuyu), in cultural-historical time, is nested within the cycle of other components, including genes, in evolutionary time. Although the frequency and scale of the cycles may be roughly distinguished, it makes little sense to separate language into an innate and an acquired component; both are parts of inherited resources" (17; emphasis added).

For Caporael, the concept of "repeated assembly" is crucial primarily due to its potential to explain the evolution of human forms of "sociality." In her approach, sociality would be viewed as derivative of several basic types of "repeated assembly," which are the product of a nested hierarchy of life's functional organization, whose respective levels perform the function of the "interface" between individuals and their habitat. These levels include: dyad, task team (e.g. family or a group of people working together), $\operatorname{dem}^{147}$ (a base group facilitating

147 The term deme - derived from the Greek demos - is used in biology to denote a populace comprised by individuals living close enough to each other to ensure equal chances for all to form pairs and reproduce (Hull 1990, 433). In anthropology, the counterpart to deme would be probably "tribe," while in the case of macrodeme it would be "tribal group." However, since the original terms have a more universal meaning (which is important for later discussions), we have decided to call them (respectively) "base group" and "macro group." 
direct contacts among members), and macrodeme (or a macro group). These four "core configurations" do not represent groups in the literal sense, but they rather represent basic, general types of human interaction. They are "crucial" not only because they have been developed in the hunter-gatherer period of human history but also because they are ceaselessly reproduced, from generation to generation, in the course of people's everyday activities. This stems from the fact that these configurations are a function of the physical parameters of human morphology and ecology. Consequently, each of them serves to realize a specific "modal task." "From a strictly evolutionary historical perspective," Caporael argues, "core configurations are affordances for the evolution of proper functions" (23).

A given affordance allows for the emergence of specific types of "repeated assembly" described in terms of their "proper function." However, this relation is not of a necessary nature - proper functions ${ }^{148}$ are not automatically created because of the existence of some kind of a configuration. Still, once developed, they can be transferred both to lower strata of the hierarchical organization of life, or combined with functions typical for higher ones. For example, the development of language certainly improved micro coordination in dyads, but that achievement - when combined with standardized language - streamlined operations at the level of task groups, helping them in acquiring their proper functions.

The basic elements of the model proposed by Caporael are listed in table 4.1. The descriptions of "modal tasks" included in the table would suggest that the model applies largely to primal communities. However, such a conclusion would entirely contradict both the assumptions made by the author, and the potentials inherent in the model itself. As mentioned above, Caporael sees the key configurations she has distinguished as a reflection on fundamental "existential pressures" proper to humanity, whose realization always takes place in a defined specific social environment. Thus, since they were created in the course of evolutionary processes, they constitute the basic modus operandi of humanity

148 Caporael does not use the category of function in the normative sense (i.e. as functional vs. dysfunctional), but in the mathematical one, which emphasizes the dependence of a specific result on having fulfilled the requirements necessary for it to occur. Some types of "repeated assembly" have no normative function because they are based on imagined relations (e.g. superstitious behavior among pigeons in Skinner's experiments), while others can have a normative function for some time and then lose it, without making it ineffective due to force of habit (cf. the previous chapter). 
Table 4.1. Core configurations (after Caporael 1995)

\begin{tabular}{|c|c|c|c|}
\hline Core configuration & Group size $^{\text {a) }}$ & Modal task & Proper function $^{\text {b) }}$ \\
\hline Dyad & 2 & $\begin{array}{l}\text { Sex, infant interaction with } \\
\text { adults \& older children }\end{array}$ & Microcoordination \\
\hline Work/family group & 5 & $\begin{array}{l}\text { Foraging, hunting, direct } \\
\text { interaction with habitat }\end{array}$ & Distributed cognition \\
\hline Deme (Band) & 30 & $\begin{array}{l}\text { Movement from place to } \\
\text { place, general processing and } \\
\text { maintenance, work group } \\
\text { coordination }\end{array}$ & $\begin{array}{l}\text { Shared construction of } \\
\text { reality (includes folk } \\
\text { psychology), social } \\
\text { identity }\end{array}$ \\
\hline $\begin{array}{l}\text { Macrodeme } \\
\text { (Macroband) }\end{array}$ & 300 & $\begin{array}{l}\text { Seasonal gathering, exchange } \\
\text { of individuals, resources and } \\
\text { information }\end{array}$ & $\begin{array}{l}\text { Stabilizing \& } \\
\text { standardizing language }\end{array}$ \\
\hline
\end{tabular}

a) Except for the dyad, the numbers quoted here ought to be considered as approximations with a margin of 33per cent.

b) Different proper functions may emerge in individual configurations, but only one example is provided here.

as a species, transferred in time and recreated in various, entirely dissimilar areas of human activity. To illustrate this thesis, Caporael refers to the analysis of the organization of academic life done by David L. Hull (1990). ${ }^{149}$

Hull's work itself constitutes an extremely interesting example of numerous recent attempts to adapt the language developed in natural sciences to analyzes of processes that are, strictly speaking, social. ${ }^{150} \mathrm{He}$ adopts the perspective of the theory of evolution to analyze the development of science, indicating the existence of many analogies between concepts used by the theory of evolution, and ones that could be used to characterize academic work. Among other things, he draws attention to the importance of the "demic" structure of science for the so-called fitness of particular scientific concepts. According to him, scientists are

149 She mentions his work in the introduction as a source of inspiration for the model she has developed.

150 Another example is a 1980s trend in culture-focused research, in which changes of culture are analyzed in terms of "memes" (the basic replicators of culture, similar to genes, which are the basic replicators of organisms), which compete with each other for space in human minds (cf. e.g. Blackmore 2000). However, this metaphor refers to concepts that are considered in contemporary biology an instance of misusing the theory of evolution because they exemplify the "individualization" of the populationbased model that lies at the heart of evolutionary theory. 
divided into "populations" that devote themselves to studying specific segments of reality by using similar ideas or approaches. "Initially, in the history of science," Hull remarks, "scientists work in relative isolation from their contemporaries. They build on past work but not band together to pursue joint research. Rather rapidly, however, the demic structure of science materialized and continues to characterize science to the present" (23). This creates a specific problem since despite scientists competing for citations, prestige, and being "first" - in order to achieve these highly individualistic goals they are forced to cooperate.

This problem - which also emerges in theories of evolution, where altruistic behavior remained an unsolved puzzle for a long time $e^{151}-$ is solved in the world of science thanks to the specificity of the organization of academic life. The "demic" structure of science defined by Hull comprises individual scholars, research groups, "conceptual demes" (or "schools"), and seasonal meetings of scientific associations. Taking into account the fact that individual scientists usually make use of works written by their predecessors (while reading Hull's book, the reader forms a dyad with him), clear and detailed parallels become traceable here with the "core configurations" defined by Caporael on the basis of analyzes of hunter-gatherer communities.

In both kinds of collectivities, task teams are the level of organization that makes it possible to exchange, compare and combine information gathered individually by particular members of the task team after the division of tasks. This process contributes to the development of a specific "approach" at the level of the scientific "conceptual deme," or the tribe, in turn drawing on conceptual schemas already developed at this level. The level of "school" or tribe is the one on which resources and information gathered by task teams are merged. It is also

151 Today's theories of evolution demonstrate that altruistic behavior can be explained by "inter-group selection": whereas intra-group selection tends to eliminate altruism (since inside their own group altruists lose to other individuals), inter-group competition would actually promote it. This intuition was already voiced by Darwin himself in The Descent of Man (1871): "It must not be forgotten that although a high standard of morality gives but a slight or no advantage to each individual man and his children over the other men of the same tribe, yet that an advancement in the standard of morality and an increase in the number of well-endowed men will certainly give an immense advantage to one tribe over another. There can be no doubt that a tribe including many members, who, from possessing in a high degree the spirit of patriotism, fidelity, obedience, courage, and sympathy, were always ready to aid one another, and to sacrifice themselves for the common good, would be victorious over most other tribes; and this would be natural selection" (after Sober \& Wilson 1998, 5). 
the level on which the social identity of individuals is developed. Finally, in the case of both scientists and members of hunter-gatherer communities, seasonal meetings at the level of the macro group offer an occasion to exchange myths, gossip and information about more distant regions or groups. Such meetings also help standardize language (ethnic one or sociolect), exchange people (e.g. women among tribes, or new $\mathrm{PhD}$-holders among research groups), and offer an arena for undertaking "competitive games."

Indicating similarities between the organization of scientific life and that of hunter-gatherer communities does not aim to promote another striking metaphor of the "tribal" character of academic life. The intention would not be to mechanically translate a language used to describe certain phenomena (as developed in one area of science) into another language used to describe different phenomena, but to consider the extent of the "tribal character" of political, artistic or economic life. Hull's book itself demonstrates that his conceptual scheme allows to produce explanations and formulate hypotheses that go far beyond analogies between characteristics of two kinds of collectivities. Similarly, despite referring mainly to examples from hunter-gatherer communities, Caporael is convinced that her model could be adapted not only to describe other types of collectivities but also to analyze processes of ontogenesis as well as processes occurring at the level of society as a whole.

We share this conviction. However, in order to fully estimate the possibilities inherent in this model it is necessary to "set it in motion." Thus, we first need to closely examine its core configurations, especially the way in which they correspond to processes of socializing discussed in previous chapters. In other words, it is paramount to adopt this model - sketched in very general terms by its author - to the kind of matter that is typical for sociology, i.e. key issues analyzed by this discipline. Only then would it become possible to assess what could be gained from making the strictly theoretical assumption that lies at the heart of this model, namely one that involves analyzing social life in terms of its hierarchical organization, rooted in specific configurations of interaction, whose particular levels are focused on diverse tasks and perform different functions.

\subsection{Dynamics of social life}

Among the four configurations distinguished by Linnda Caporael, the dyad is the smallest unit of the "repeated assembly" of relational linkages - terms in which human actions could be analyzed. The basic function of the dyad is to produce micro coordination between the two elements that comprise it. It is vital to understand that these two elements do not have to be both human - it is possible 
for a dyad to be formed by a person and an object, or any living being. In each case, the repeated relational linkages between positively reinforced (selected) elements of the dyad (e.g. two people, man and hammer, dog and dog owner) cause them to form a coordinated task team. It is also crucial that this kind of micro coordination is derived from intense, close interactions between individual entities that make up the dyad.

The use of the term "interaction" in this context might raise objections due to the above definition of dyad as possibly comprising not only two people but also a person and an inanimate object or a non-human living being. However, it is justified for at least two reasons. First, non-human elements of dyads are not merely passive objects, on which people act, but are responsive, at least by way of revealing certain properties. ${ }^{152}$ An overly "intimate" interaction between my finger and a hammer can lead to painful injury, while "interacting" with a stray Rottweiler demands taking entirely different cautionary measures than contacts with our own lazy spaniel. Second, in many cases the objects we use are vehicles of somebody else's intentionality (often accumulated for generations), while other beings also have their own experiences gained in contacts with people, or - to put it in other words - they have been "socialized" earlier on. While interacting with them, we need to adapt our reactions to the intentions of the people we do not know, the intentions "materialized" in shapes or properties of objects, and the ways in which animals react.

Two things need to be emphasized here. First, relational linkages characteristic for this level do not exist in the same sense as the "objects" that comprise their elements - their existence needs to be continuously sustained. ${ }^{153}$ When different people, tools or dogs enter the dyad, they necessitate reconstituting these linkages. Second, once established, these linkages change the "nature" of the objects that form them. For example, a hard stone becomes a "hammer" when used in a specific way, a man with an axe becomes a woodcutter, while an infant becomes a cowed baby in contact with a domineering adult. However, these changes are limited to the particular dyad - in the context of others, a stone could still be a stone, an axe could serve the function of propping the door, while a cowed child could have its say.

152 The general logic of scientific efforts is similar, consisting for example in questioning nature (e.g. in laboratory experiments), in response to which nature (sometimes) answers.

153 Certain colloquial phrases like "being out of practice" signify being "out of" a certain dyad. 
What we encounter in a dyad is the mutual adjustment of its elements' actions and reactions within the framework of a repeatable "task." It is the character of a specific "task" that is responsible for the particularity of relational linkages created in a given dyad. Consequently, actions of individuals become complementary, making the dyad function - in certain conditions - "as one person." In other cases, it is the person and the object's properties that become complementary, making, for instance, the scalpel held by a surgeon become an "extension" of her hand, or giving the impression that a rider is "physically joined" to her horse. A relational linkage developed by a pair of lovers who - according to what selfhelp books claim - ought to learn about their bodies so as to be able to climax together, is different from that formed by a pair of professional dancers for whom the knowledge of the partner's body constitutes the condition for achieving a harmony of movements, which is assessed by jurors. Yet another kind of bodily knowledge is required in the infant-adult dyad, whose basic task is to keep the baby alive. For the adult, this means learning how to adequately read the signals sent by the child, while the child needs to accept that, for example, not every single need will be immediately satisfied.

What links the above examples of micro coordination is the fact that all establish a special kind of connection between the dyad's elements, which - as we argued in the previous chapter - has a complementary character. We argue that such connections are accompanied by a limitation of individual freedom that stems from the subordination of the actions of the dyad's members to the realization of a specific "task." Let us notice here that this limitation does not have to be an effect of the partners' emotional engagement. ${ }^{154}$ It could also have a more instrumental character, involving for example willful, "rational" surrendering of a part of personal freedom to the "whole" or it can be established by one of the interacting actors by force. The coordination of actions achieved will have a different character in each case, but is invariably related to dependence, and - in consequence - to the specific "enslavement" of both parties, which are locked in mutual dependence. Simmel called attention to this, remarking that in a dyad "each of the two feels himself confronted only by the other, not by a collectivity above him" (1950, 123).

154 It does not necessarily have to be positive. Stanisław Lem came up with the nonexistent word "gniewiść," signifying a mixture of love and hate. One example of this is provided by the relation between a torturer and his or her victim. Similarly, gaining awareness of the complementarity of emotions and actions constitutes the first step in therapy for wives of alcoholics. 
Interactions in dyads constitute the lowest level of the organization of social life, from which the second type of "core configuration" grows, namely the "team." This term covers various kinds of social associations, including family, task teams, and people working in one organizational cell. Teams usually comprise 5-7 people and tools. ${ }^{155}$ Whereas the basic function of interactions within dyads is to facilitate mutual adaptation among constituent elements, the level of team coordination is defined by particular tasks realized by specific groups in direct contact with their habitat - initially, only the natural one but along with increasing complexity of social life also comprising institutions (in the strict sense) and technological infrastructure, created at higher levels of coordination.

At this level of hierarchical organization, a more complex reality emerges one that nobody can fully control. ${ }^{156}$ As a result, cognition becomes "distributed"157 - each member of the cooperating group of people has direct access only to some part of the other members' experience, and no one possesses all knowledge. Moreover, each group member remains in a certain relation to some part of the larger social environment (e.g. father to men, mother to her family, children to their peers), which additionally differentiates views of reality. As a result, individuals not only differ from each other in terms of knowledge but also do not know what others know. Access to the knowledge possessed by others is therefore obtained indirectly through becoming engaged in joint action. As a

155 It is worth drawing attention here that even brief engagement in a "task" can often transform a collectivity into a team understood in this way. For example, a gathering of people in a lift does not constitute a group, but when the lift malfunctions we can observe the development of "cooperation around a shared task," which transforms the people involved into a task team.

156 For example, interactions in families are usually organized in pairs (father-mother, parent-child, child-child), so that other family members do not participate in them.

157 The term "distributed cognition" denotes a specific approach to the study of cognitive processes. Developed since the 1980s, it assumes - in broadest terms - that cognition is a function not only of the intellectual properties of individual minds but also interactions between minds and elements from their surroundings: other people, objects, events occurring at different moments, etc. Therefore, depending on the context in which it is used, this term could mean at least two things: first, it might emphasize the characteristic "distribution" (or dispersion) of information among elements that form the "cognitive system"; and second, it may underscore the fact that the portion of information that belongs to one element of the system is made available to other elements in the course of interactions that consolidate the entire "cognitive system." Cf. a broader discussion of this in Hutchins 2000; Cole \& Engestrom 2001. 
result, such access is always selective because it is determined by the character of the actions taken.

Despite being "distributed" in a variety of relations, adaptations, experiences and actions - which leads to a lesser intensity of "repeated assemblies" than in dyads - a team can nevertheless create its own specific representation of a certain part of reality, one that primarily involves developing the vision of the task it is supposed to perform (regardless of whether it is the growth of the family, or drafting a quarterly balance) and defining its interests in terms of interests identified in its immediate social surroundings (i.e. the configuration from the next level of hierarchical organization). This representation is specific to the team also because its final shape is determined by the properties of those elements of "distributed cognition" that are introduced by each individual member of the team. ${ }^{158}$ It is developed in the process of communication between members of the group, in the course of which at least some portions of "distributed cognition" are spread, modified and negotiated within the team. At the same time, its existence constitutes the condition for effective realization of tasks faced by the group, because referring to shared knowledge makes the meanings of facts and opinions (at least those related to shared tasks) comprehensible to all members of the team. ${ }^{159}$

Let us note here that - at the level of the team - the concept of "distributed cognition" reveals for the first time this feature of social reality which sociologists have called the "primacy of the whole over its individual components." It is especially in Durkheim's theory (cf. also Harré 1980) that this feature constitutes the basic aspect of social phenomena. ${ }^{160}$ This is because representations of reality formed by teams may emerge from knowledge possessed by an individual but

158 If the group includes - for example - a dominant person who believes in a conspiracy theory, the team's main task may become to defend itself from the outside world.

159 In this sense, the nature of "distributed cognition" is basically similar to that of "social representations," although the latter term - developed by Serge Moscovici (1981, 1983; Moscovici \& Farr 1983; Trutkowski 2000, 2002) - certainly has a broader meaning. Clearly, each term emphasizes a different component in the process of negotiating common knowledge: the former takes the distribution of information as its starting point, while the latter takes it as its final effect. Nevertheless, both refer to the same process of creating social knowledge.

160 Actually, its existence manifests already at the level of the dyad - the lowest unit in the hierarchical organization of social life - through a specific limiting of individual freedom. However, as Simmel has demonstrated (1950, 122-144), the dyad has certain specific characteristics that obfuscates the social character of this kind of relationship. 
cannot be reduced to it. They form a new quality that emerges in the process of negotiating elements of this knowledge among members of the group. They are social not only due to their genesis but also because of their "ontological status."

It is also at the level of the team that the fundamental process of socializing new members occurs in relation to both newborns, as in the case of children socialized within families, and newcomers who arrive from outside to strengthen the team and are subjected to secondary socialization. ${ }^{161}$ As theoreticians of "distributed cognition" emphasize (usually by quoting Lev Vygotsky), in both cases the process is carried out by acting in a specific habitat, whose characteristics determine both the scope of actions undertaken by team members and the contents of "distributed cognition" handed down within a given team. For this reason alone, the effects of a child's socialization are bound to differ between those families that have only a TV set and a refrigerator and those that possess numerous other objects, that are - as Pierre Bourdieu put it (1986) - well-endowed with cultural capital. Similarly, "distributed cognition" differentiates socialization effects among middle and working-class families, manifesting for example in parenting values (cf. Kohn 1959; Schooler \& Kohn 1983).

The process of socializing of distributed cognition at the level of teams depends not only on the experience of individual members and the nature of the tasks they seek to complete, but also on processes occurring at higher levels of life's hierarchical organization, where the core configuration is a larger deme, called here base group. In sociological terms, this would correspond to the concept of community. The basic task of deme is to coordinate work or - to put it in broader terms - actions undertaken at the level of teams. In contrast to some self-imposing associations, this "managerial" task is not realized through direct management of work done by the teams, but primarily through creation of comprehensive images of reality, which cover both the "classifying" of reality in terms of specific objects, phenomena and processes, and the "endowing" of distinguished elements with specific properties, as well as indicating the actions that are generally imaginable, possible, desired, tolerated or forbidden. ${ }^{162}$

The key function of these images of reality is to make distributed cognition coherent in such a way that certain experiences of the collectivity's members can become not only the kind of knowledge that is shared by all, but also the one

161 Krzysztof Konecki (1992) provides an excellent analysis of this process in the context of a community of workers in an industrial plant.

162 In Caporael's terminology, this aspect of images of reality is referred to as "folk psychology." 
in which everyone knows that everyone knows that... In other words, images of reality include and simultaneously constitute everything which is taken for granted, i.e. which does not demand any justification in the course of interactions. It needs to be emphasized here that, in accordance with assumptions made by Caporael, these images of reality are not - as has been commonly assumed in sociological practice ${ }^{163}$ - constructed directly from individual experience, but rather constitute the effect of transforming and adapting representations developed in team configurations. ${ }^{164}$

It is at this level of the organization of social life that a group of variously aged people of different sexes who live under the same roof acquires the name of "family" 165 or - in a different context - people engaged in work on this book become the Department of Social Psychology. It is also at this level that ways of solving problems, developed by teams in the course of performing specific "tasks," acquire a normative status, turning into "principles of appropriate behavior," "justice" and "methodology" - or, more generally, into criteria of beauty, good and truth (Giza-Poleszczuk 1991a). The inclusion of action patterns developed by individual "teams" into the set of images of reality constructed at the level of community must involve explaining to other members of these groups why they need to undertake such actions and not others, and "why things are what they are" (Berger \& Luckmann 1991, 111). Moreover, these explanations ought to include not only indication of pragmatic efficiency of certain solutions, or objective features of specific "things" 166 but also references to outside justifications such as tradition, the sacred or rationality (Weber 1958). Such justifications constitute the essence of legitimizations that make certain patterns of action not only objectively available but also subjectively acknowledged by larger circles in

163 This in not limited to practice only. Berger \& Luckmann have laid the theoretical foundations for the development of a constructionist approach (1991), in which the "social production of reality" is depicted as a process that takes place in interactions among individuals.

164 This relation naturally has the character of a feedback loop: at the team level, representations are developed within the framework of a given image of reality, which is enriched with new experiences that, in turn, contribute to its change.

165 It may happen that representations produced by a certain "team" are rejected at the level of community, making some kinds of groups be tagged as "shacking up together," with children raised in them called "bastards."

166 Such explanations are directly related to experiences from the level of that team which developed a specific solution or thing. This means that they could be questioned by members of other teams. 
a given community; ${ }^{167}$ thus, they can become the fundamental tool for the realization of the task of coordinating work in teams.

This process of legitimization is naturally arbitrary, because it utilizes already existing elements of images of reality - elements which provide an interpretation, not description of reality - whose roots lie in all-encompassing visions of the world created in the act of "foundational holism" in a given community. In reference to the discussion contained in Chapter Two, it could be said that the process of developing images of reality must be preceded by the process of ascertaining, at the level of community, of such general cognitive frameworks that make experiences gathered in dyads and teams meaningful, thanks to which these experiences are in turn legitimized. Regardless of whether such general cognitive frameworks are rooted in religion, based on scientific claims, or grounded in political views, accepting them is a sine qua non condition not only of the process of introducing coherence to collectively developed images of reality but also of the very functioning of individual minds. ${ }^{168}$

The above statements carry several vital consequences, which demand further examination.

First, they entail the necessity to treat the described model of hierarchy-based organization of social life as one that accounts for its dynamic, not its genesis. In other words, the fact that the lowest level of organization is that of dyad does not allow to draw the conclusion - found at the foundation of today's widespread interpretive paradigm - that interactions between autonomous individuals constitute the basic level from which particular, historically known forms of sociality emerge. In the model adopted here, the level that is essential to the process of socializing is that of deme - i.e. community - and not the one of individual interactions. This conclusion is rooted not only in relations between images of reality developed at the level of the base group and cognitive processes occurring at lower levels of organization but also in other assumptions made in this model, including the following one: "groups would reproduce by fissioning, not by dispersing individuals or dyads" (Caporael 1995, 21). It follows from this that the mechanism at the base of social change ought to resemble one of division of

167 As Peter Berger and Thomas Luckmann rightly emphasize (1991), "knowledge" is always ahead of "values" in the process of legitimization. It needs to be recalled that in the model assumed here "knowledge" comes from a lower level of the organization of social life than the one on which "values" are created.

168 Let us recall here that, in the philosophy of science, this kind of interpretation is ascribed to the so-called Gödel's theorem, i.e. the claim that within every system there are true statements that cannot be derived from that system's axioms. 
cells rather than the evolutionary growth of individually developed inventions. New forms or kinds of sociality do not emerge thanks to individual innovation with novelty gradually spreading in wider circles ${ }^{169}$ - but by new communities "fissioning" from already existing ones, the former being organized around more or less different images of reality than their predecessors. A classic example of such a process is provided by Max Weber in The Protestant Ethic and the Spirit of Capitalism (1905).

This does not entail, however, a total negation of the influence of individuals on social changes. Protestant ethics would not be created, after all, or would be completely different if it had not been for Calvin, Luther or other "dissidents." Nevertheless, this fundamentally alters the very basis for explaining the dynamics of social life. In light of the assumptions we have made before, its dynamism needs to be interpreted in terms of forming subsequent (or parallel) "foundational holisms," ${ }^{\prime 10}$ which are collectively created and supported through actions taken by members of a given community or society. It thus becomes vital to ask about the factors and mechanisms lying at the foundation of the process of reproducing groups, because it is the emergence of new communities that provides impetus to the creation of new images of reality (or to the introduction of far-reaching modifications in already existing ones), and contributes to the transformation of this reality in the course of actions undertaken by members of those communities.

Second, interpretations of reality accepted within groups play a fundamental role in the process of forming social identities, which is the second - besides the development of images of reality - important function of the base-group level

169 This has been unequivocally confirmed by research done within the framework of so-called dynamic social psychology (cf. Vallacher \& Nowak 1997; Nowak 1996). It shows that processes occurring in differentiated systems lead to the grouping of those individuals who share similar views or patterns of behavior. To put it metaphorically, individuals who have different views than those held by people from their immediate surroundings (or who differ in terms of patterns of behavior) are incapable of "surviving" in such an environment - they would need to change either themselves or their surroundings.

170 We put this term in quotation marks because - especially today - these foundational visions rarely have the character of an overall negation of the dominant image of the world. What we usually encounter is a desire to introduce or just accentuate one or several assumptions (as in the case of the move from the normative paradigm to the interpretive one in sociology), or those "values" that members of the base group consider to be crucial in a certain area of reality (as in the case of the "mushrooming" of various groups within feminism; cf. Gromkowska 2002, 51-78). 
of human interactions' core configurations. The positioning of the process of developing social identities at this level of the organization of social life might be surprising, especially in light of numerous works on the subject of its formation, which ascribe the task of constructing identities not only to so-called small groups (their counterpart in this model would be teams) or dyads (this is, after all, the meaning of Giddens' claim about the importance of so-called pure relations for the formation of identity), but simply to the individual. It ought to be recalled, however, that the basic tool for the formation of individual identity is difference (Jacobson-Widding 1983; Tajfel \& Turner 1986), because the answer to the question "Who am I?" always implicitly contains an answer to the question "Who am I not?" Establishing of the dimensions of differentiation is an inseparable element of the collective process of constructing reality.

Thus, regardless whether the answer is formulated in terms of psychological states and properties ("I am intelligent;" "I am possessed"), or in terms of representing specific groups and social categories ("I am a member of the Bison Club;" "I am a sociologist"), the ideas that stand behind them refer us back to those aspects of our experience that have to be first selected as crucial by the community in order to become meaningful to us in a personal way. What is more, if collective processes of constructing reality include not only classifying its aspects but also interpreting them, it is the differences in interpretation that constitute the fundamental line separating both the in-group from out-groups, "our world" from "their world" or the meaningful from the meaningless. In this way, they define the basic frame of reference for meanings ascribed to individual experiences, choices and actions. ${ }^{171}$ That is why they constitute the basic framework for developing social identity.

Third, due to the arbitrary character of each image of reality, it is the question of its sustainment that becomes paramount. As Peter Berger observes in his analysis of religion as a specific case of such an image, "[w] orlds are socially constructed and socially maintained," which means that "[t]heir continuing reality, both objective (as common, taken-for-granted facticity) and subjective (as facticity imposing itself on individual consciousness)" $(1967,45)$ depends

171 This aspect of the concept of social identity is particularly emphasized by Manuel Castells: "By identity," he writes, "[...] I understand the process of the construction of meaning on the basis of a cultural attribute, or related set of cultural attributes, that is/ are given priority over other sources of meaning" $(2002,6)$. The typology of identities he proposes - which includes legitimizing, resistance, and project identity - directly refers to questions related to the mechanisms of the emergence of new communities, which involves the emergence of new "foundational holisms" (as discussed above). 
not just on individual faith, but on the conformity of actions taken by his or her community's members and institutions with the fundamental assumptions inherent to that image of reality. In other words, the reality of God is confirmed by people gathering in temples, priests performing rituals and leaders leading armies against infidels. ${ }^{172}$ Similarly, the reality of the hierarchical organization of social life would be confirmed by scholars researching dyads, teams and demes, as well as institutions funding research and Departments of Hierarchical Organization of Social Life opened at institutes of sociology, etc. ${ }^{173}$

Since the maintaining of a given image of reality relies mainly on actions that conform to it (which sometimes demands huge efforts on the part of individuals), communities have to develop some means of social control. Disciplining methods include a wide range of measures: disapproval expressed in non-verbal communication, verbal condemnation, societal ostracism, punishment by isolation, and even physical extermination. Their application depends on the degree of the misdeed, which, in turn, is a function of the weight of the "offence" (judged from the perspective of a given image), the force with which the image is maintained by a given society, and the degree of threat posed by the violation of a particular image to its sustainment in the community.

A more detailed analysis of consequences entailed by these findings for the understanding of how contemporary society functions is provided in the last chapter. At this point, however, we shall return briefly to the description of Linnda Caporael's model. Its fourth and final level of core configurations of interactions is that of the macro group. This level is characterized by the slowest

172 Perhaps, this is what Durkheim had in mind when he wrote that "gods can no more do without their faithful than the faithful can do without their gods" (2001, 257-258).

173 The dispute about the existence of social classes, which rekindles in sociology from time to time, convincingly demonstrates that certain entities, which seemed very real several decades ago, today begin to appear increasingly "transparent." Still, nobody seems to be questioning the existence of "values," which are invoked not only by sociologists, but also by journalists, politicians and common people. The reality of bacteria, which most of us have never seen, is certainly sustained by hosts of doctors, who treat diseases caused by these microorganisms, by public health experts who warn us against them, and by the habit of washing hands and fruit, enforced since childhood, etc. We do not wish to argue in favor of the material inexistence of certain properties that indicate class affiliation, of certain actions and beliefs that operate as indexes of value, or of certain small beings called bacteria. Still, their material existence does not constitute a sufficient or necessary condition for their coming into existence in the social world. 
tempo of "assembly", while its fundamental aim is to organize exchanges in communities: exchanges of women or, more broadly, people, as well as information, goods, etc. The macrodeme should not be identified with what is traditionally understood as society. It is rather the domain of interactions, established at periodical gatherings of communities or its representatives. In this sense, examples of macro groups would include, on the one hand, periodical meetings of tribes for the purpose of potlatch, conferences held by scientists sharing findings in their discipline, gatherings of members of parties or associations, during which they choose their authorities and programs, and - on the other hand - regularly held fairs (including church fairs), regional nobility councils, parliamentary sessions etc. The proper function of such assemblies is to standardize language, which involves not only its homogenization within a given ethnic community, or systematization of the jargon employed by a given professional circle, but also or perhaps primarily - the homogenization of concepts used to describe reality, and their related meanings.

Because the fundamental task of the macro group is to facilitate exchanges, standardization of language must include the determination of their basic metrics, i.e. the "currency" used to define the value of exchanged goods. Whether these are seashells or banknotes, numbers of citations or sophistication of lectures, they need to be more or less consciously accepted by all communities that are part of the macro group. Rules of exchange are also subject to standardization, taking the form of common law (as in the case of the principle "eye for an eye, tooth for a tooth," which nevertheless allows for using specific equivalents) or of an elaborate legal system that involves institutions whose tasks include the prosecution of violations of these rules (as in the case of today's Hague Tribunal informed with a set of regulations defining the concept of war crime). Such rules of exchange standardize the course of "competitive games" played by members of particular communities at the level of the macro group.

Regardless of their specific character, all four levels of core configurations of interactions share certain features. It would be fruitful to distinguish them here.

First, they describe levels of interaction, and not particular groups. Although the discussed model clearly asserts that social life ought to be analyzed in collective terms, and though descriptions of particular levels often feature terms referring to group categories traditionally distinguished in sociology, one ought to remember that these categories are in no way meant to classify such collectivities. The latter should be treated rather as an arena or environment for interactions that occur within it. The question whether a particular phenomenon 
analyzed by a researcher is related to a team, a deme, or a macrodeme ought to be answered separately for each case at the empirical level. ${ }^{174}$ What is more, in particular empirical contexts each level of interaction may engage representatives of different, "substantially" distinguished social groups, which may simultaneously be members of different, parallel "communal" levels of assembly. ${ }^{175}$

Second, it follows that the basic unit of analysis in such a model is not constituted by individual people or groups as such, but by "repeated assemblies" of interaction, which include not only people but also objects (artefacts). Moreover, such assemblies have to be repeatedly created anew by way of recreating relational linkages connecting their individual elements. It also needs to be recalled that each such "recreation" is concrete, specific, and thus unique. We never eat the exact same breakfast, even when we always have croissants with jam or fried eggs; every day at work is different, even if we spend the entire day postmarking envelopes. This means that a source of changeability is hidden in the very rhythm of life, in the pulsation of "repeated assemblies," because if every act is different, it has different consequences. In this sense, the horizon of a given relation (and of actions that arise from it) changes, expands or narrows also as a result of practice and shifts of context. ${ }^{176}$ Categorizations encapsulated in language express a high degree of probability in terms of accurately describing reality rather than offer any actual classification and account of its elements. They are always temporary and contextdependent (situationally or historically), although one should never forget that despite their provisional nature they might make it difficult for language users to perceive something differently than through the lens of their own language.

Third, the assumption that "repeated assemblies" of interaction constitute the basic unit of analysis entails the necessity to scrutinize phenomena occurring at any level of the organization of social life in terms of units of coordinated activity. ${ }^{177}$ This radically departs from traditional sociological approaches to the

174 At the level of empirical research, it becomes also necessary to determine the size of individual "collective environments," i.e. teams, deme and macrodeme.

175 For example, members of Warsaw's City Council should be classified - at first glance as a kind of macrodeme, because they represent various kinds of "communities" in the form of particular political parties. However, in certain contexts - e.g. if we wish to study the functioning of the Association of Polish Cities - they ought to be treated as a kind of "community" that creates its own image of reality, its own modes of legitimization, and a distinct identity.

176 This aspect of reality is reflected in commonly used expressions, e.g. "and then the play changed into a drama" or "as soon as he arrived, I was all fingers and thumbs."

177 This approach has been broadly adopted in many concepts of management developed today. 
concepts of institution and institutionalization. The former needs to be regarded in terms of processes rather than structures, while the latter demands considering the question of how the level at which institutionalization takes places is nested in actions taken at a lower level.

For example, when analyzed as a "system of coordinated actions", a company would have to be defined as a process of transforming materials into end products. This process occurs thanks to the recreation of rhythmically repeated relations between people and things, between particular individuals and particular objects. Individuals are part of this process, aspectually "complementing" operational schemas (relational linkages) and developing their specific adaptations in dyads. In this light, individuals are not actors who perform their roles, or pawns in structural divisions; rather, they are part of a dynamic system whose functioning may be influenced by both their specific skills, the use of which is assumed by the system, and those skills that, from the perspective of the functioning of the entire system, seem irrelevant or might be even disadvantageous. ${ }^{178}$

Similarly, when analyzing processes of institutionalization, it needs to be remembered that the concept of "nested hierarchical organization" (which lies at the foundation of the entire model) means that sets of coordinated actions from higher levels do not function spontaneously since their operation has to be "nested" in lower layers of the organization of social life, comprised by their own proper sets of coordinated actions. ${ }^{179}$ And conversely, since higher levels of coordination constitute a sort of direct "environment" for lower ones, the situation when they do not adequately perform their function of the "interface" with the habitat bears consequences for the functioning of lower levels of organization,

178 This is why the theatrical metaphor is not entirely accurate, despite the fact that it helps to grasp specific aspects of human activity. People do not play their roles in accordance to a fixed script, but rather enter into systems of coordinated actions. It suffices to alter at least one element of such a system (e.g. when an object is displaced from its proper position, or when a new figure appears on the stage) to initiate the process of adapting roles (or, in extreme cases, of completely changing them) in response to the system's new properties.

179 It is worth to recall at this point the recurring phenomenon of postcolonial societies' return to tribal modes of organization upon being "relieved" from alien institutions that usually operated using naked violence. A democracy that is not authentically rooted in the units of lower order (families, local societies, and free associations, which constitute the "interface" between individuals and state) cannot be an effective tool for making the "collective will" common. 
which have to establish their own direct contacts with the habitat. ${ }^{180}$ Numerous consequences neglecting such dependencies can be traced, for example, in the Polish transformation of 1989. The institutional reforms introduced in a topdown manner were not appropriately nested at lower levels of the organization of social life, although they simultaneously initiated such processes of institutionalization that, in turn, modified - unfortunately, usually in an undesirable direction (cf. Marody \& Wilkin 2003) - the functioning of higher levels.

Making processes of institutionalization dependent on already existing systems of coordinated actions means that it becomes highly probable that newly emergent systems "accumulate" such patterns of interaction that have been developed within prior systems. ${ }^{181}$ It stems from the fact that processes of interaction both bind people together and emerge from already existing relations. This entails, fourthly, the necessity to devote closer attention to the creation of linkages among individuals in the course of these interactions. The problem becomes particularly significant on those levels of coordinating interactions that are higher than the level of dyads, i.e. the levels on which individuals take actions within clearly defined groups (teams, communities or macro groups).

We shall revisit this problem further in the book. However, we wish to draw attention here to the fact that although the model we follow assumes that new linkages are established always on the basis of already existing "products" of core configurations of interactions - images of reality, social identities, or language the use of such "repeated assemblies" to establish new linkages between people at the same time creates an additional mechanism that lends more dynamism to social life. Its significance increases along with the changes of historically known forms of sociality.

\subsection{Analyzing processes of historical changes}

At first glance, the discussed model's key concept of a "repeated assembly of interactions" may seem close to the concepts around which sociology's two fundamental paradigms - normative and interpretative - are organized (Wilson

180 Historians of the family emphasize the special dependency between the family and the state: a weak state is usually accompanied by a strong family, and vice versa. In the former case, the lack of an appropriate "interface" forces units from the lower level to directly interact with the environment.

181 This phenomenon is often called "path dependency." Its primary effect is that processes of social change have to be analyzed in a way that takes into consideration a broader historical perspective. Problems arising from this are discussed in further chapters. 
1970), namely those of "patterns of behavior" and interactions as such. The former is linked to "assembly" by way of its iterative character, whereas the latter - through the fact that they are focused on joint actions, and not on individual behavior. Despite such similarities, however, the concept of "assembly" differs fundamentally from its predecessors, at least in several respects.

First, the above concept does not concern forms taken by individual actions ${ }^{182}$ but rather their consequences, which consist precisely in the above "repeated assemblies" created in the course of interactions. For example, micro coordination can take many forms, depending on the dyad's component, the character of adaptations and the features of the social environment (the level of teams); however, in no case can it - as we have demonstrated - "congeal" in a form transferred from one situation to another. It is not a norm, or an algorithm of action, but a dynamic system that is subject to never-ending micro changes. Second, the concept of "repeated assembly" always engages at least two elements (the dyad), which are analyzed in terms of their mutual adaptation. In other words, no room is left for the emergence of any "autonomous subject" reflexively pursuing her goals. ${ }^{183}$ The individual is always socially oriented, at least in the sense of her actions being invariably dependent, at least to some degree, on the actions of the partner(s) in interaction ${ }^{184}$ - even if the latter obediently accepts the definition of the situation created in the course of that interaction. Even when we observe a single individual, her behavior has to be considered as an effect of her past adaptations, which have somewhat shaped it. Third, the scope of these adaptations is not accidental or shaped solely by individual needs, "utility," or preferences, but rather - in line with the assumptions made here - is limited

182 In the case of the normative paradigm, the basic form for patterns of behavior is the social norm, a concept that emphasizes how a given action should be carried out. In the case of the interpretive paradigm, the focus on form is best visible in ethno-methodological analyzes, and in Goffman's analyzes of the "presentation of self in everyday life," which underscore the importance of ritual, playing roles, defining frameworks of behavior, etc.

183 This does not mean, however, that individuals cannot strive to achieve goals that are important only for them, or attempt to acquire "full" control over own actions. Nevertheless, the question of agency or of the importance ascribed to personal goals can be considered only from the perspective of consciousness, which makes it the domain of psychology and social psychology.

184 Paradoxically enough, this assumption does not differ in any way from the one made by Mead. However, symbolic interactionists clearly shifted their focus from processes of mutual adjustment of individual actions to outcomes of such processes, i.e. definitions of situation (cf. Blumer 1966). 
and defined, on the one hand, by those ways of regulating relationships among individuals that are prevalent in a given type of society on all four levels of interaction, and - on the other - by the main principle organizing social order, which determines the specific kind of society in question. In other words, the scope of adaptations is defined and limited by both ways of understanding socialization discussed in Chapter Two. Finally, or fourthly, this model could potentially explain the transformations of a once created form of sociality. This makes it a promising theoretical point of departure for describing processes of social change.

The last statement demands further expansion. At the foundation of the model proposed by Linnda Caporael, we certainly find analyzes of primary communities, which is confirmed by the vast majority of examples she uses, as well as by the descriptions of at least some tasks assigned to particular levels of configurations of interactions (cf. tab. 4.1, p. 137). Basically, one could say that Hull's work - which she quotes as an important source of inspiration - is used in her work mostly to establish the equivalence between primary communities (in the anthropological sense) and ones that are "primary" in the social sense, i.e. those that share with the former the basic function of establishing a shared vision of reality. Caporael also uses Hull's work to demonstrate her own model's potential for analyzing phenomena and processes typical for contemporary society, ultimately achieving an ambiguous effect, to which we shall return later. However, if we limit ourselves to primary communities in the narrow sense, we should pay attention to three kinds of relations - matching our findings in the previous two chapters - which may play a significant role due to the evolution of this form of organization of social life.

The first one is the relation that links a given system of coordinated actions (in this case the community) with the habitat. It is crucial chiefly because it affects to a large degree the collectivity's size, consequently affecting the transformations of its internal organization and structure. As Jared Diamond has demonstrated (1999), unfavorable features of the habitat constituted the main factor that decided about the lack of development and the survival to this day of small hunter-gatherer communities, consisting basically of multi-generation families: several adults, children and elderly moving within a territory they controlled. Describing one such primary community that still lives in New Guinea, Diamond argues that their lasting commitment to a nomadic lifestyle stems from the fact that "the region lacks dense local concentrations of resources that would permit many people to live together, and that (until the arrival of missionaries bringing crop plants) it also lacked native plants that could have permitted productive farming" (1999, 270). He concludes by saying that "[b]and numbers 
are kept low by diseases (especially malaria), by the lack of raw materials in the swamp (even stone tools must be obtained by trade), and by the limited amount of food that the swamp yields for humans" (270). ${ }^{185}$

Along with the adoption of a sedentary lifestyle, which started agriculture and animal husbandry (estimated by archaeologists to have happened ca. 10,000 years ago in particularly fertile areas), the size of population began to increase. The tribes that emerged then comprised several blood-bound groups (clans) and included hundreds of people. However, all members of the tribe would know each other and be related by blood. It is an important factor, because it decreased the probability of arguments inside the community. In other respects, the tribes would not differ much from bands. Without formal leadership, the differences in position would be still related only to sex, age, and personal characteristics. Status would not be inherited by individuals, the system of mutual obligations would not favor accumulation of wealth, and the economy would be based on the exchange of goods among families and individuals.

However, the relation between new ways of exploiting the habitat (agriculture) and the growth of population had an autocatalytic character. According to Diamond, it "catalyzes itself in a positive feedback cycle, going faster and faster once it has started" (111); furthermore, the tempo of population growth slightly exceeded that of the increase in food production. ${ }^{186}$ This necessitated searching for new means of increasing production, which in turn led - when successful - to the increase in the size of population. This process had two important consequences. First, it favored the development of tools and new methods of production. Second, it led to the emerging of centers of power, and initiated the process of social differentiation since in those collectivities whose numerical

185 In terms developed by Diamond, the band is the smallest and most "primary" (in the evolutionary sense) type of social organization, which was probably prevalent in the period of developing the "foundational holism." It would typically comprise 5-80 people, most of whom would be related. "Bands lack many institutions that we take for granted in our own society" $(1999,268)$. Territory would belong to the entire collectivity and there would be no stable economic specialization (aside from tasks tied to age and sex). Without any system of governance, leadership in the band would be informal and won only through individual abilities such as strength, intelligence and military skill.

186 According to data gathered by archaeologists, "first farmers in many areas were smaller and less well nourished, suffered from more serious diseases, and died on the average at a younger age than the hunter-gatherers they replaced" (Diamond 1999, 105). However, there were more of them, which gave them an edge in contacts with others. 
strength passed a certain threshold, kinship ceased to be the mechanism regulating relations between individuals, exacerbating the risk of internal conflicts, which caused chiefdom to emerge along with the distinction between the rulers and the ruled. ${ }^{187}$ At the same time, a separate population category emerged - i.e. clerks and craftsmen - who worked for the chieftain and were paid with tributes offered to him.

This is already a different type of social organization, because it fundamentally restructures the population and the system of forces operating within it. The egalitarian though certainly differentiated community was replaced by a hierarchically structured collectivity, in which certain categories of people occupied lower levels of the social structure, while others were elevated to the top and furnished with the authority to issue orders. This entails the transformation of the second crucial relation, namely one linking the system of coordinated actions, i.e. the primary community and the images of reality it generates, or (to employ terms that shall be used in further analyzes) its collective representations. The emergence of chiefdom was accompanied, on the one hand, by a necessary change in these representations so that they could embrace the transformation of social identities, and - on the other - a necessary legitimization of the new social order, which usually led to the transformation of the older system of beliefs into an institutionalized religion supporting the chieftain's authority.

Institutionalized religion not only legitimizes the new social order but also "brings two other important benefits to centralized societies. First, shared ideology or religion helps solve the problem of how unrelated individuals are to live together without killing each other - by providing them with a bond based on kinship. Second, it gives people a motive, other than genetic self-interest, for sacrificing their lives on behalf of others" (278). The "interest of others" a general interest - leads to the third crucial relation, which links collective representations and the habitat. Certain elements of these representations had to be modified earlier due to the emergence of actions that originated in the abovementioned development of tools and methods of agricultural production, which accompanied the shift from a nomadic lifestyle to a sedentary one. Thus, one could generally say that new actions related to the transforming of the habitat always create a kind of "anomaly" in the predominant image of reality; therefore, they need to be somewhat incorporated into it. At the same time, however,

187 Diamond makes the following comment about this process: "[w]ith the rise of chiefdoms around 7,500 years ago, people had to learn, for the first time in history, how to encounter strangers regularly without attempting to kill them" (273). 


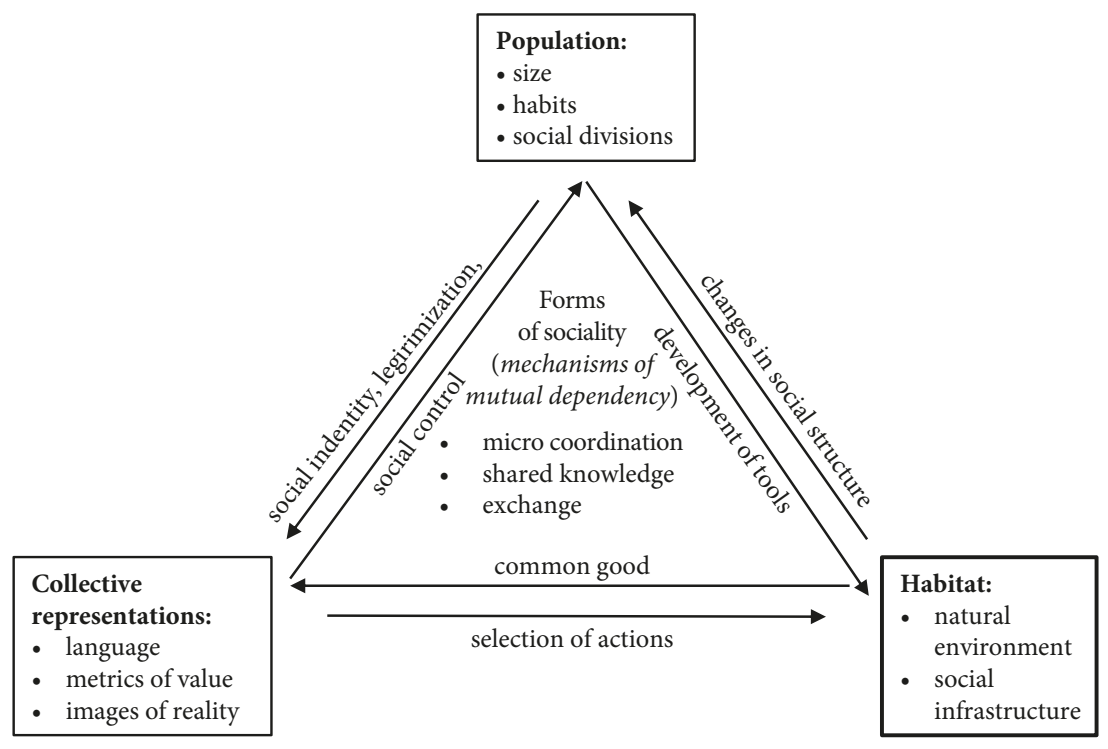

Fig. 4.1: Relations between main factors influencing processes of socializing and their changes

parallel transformations in social representations, arising from changes in the collectivity's structure, redefine the concept of the common good, which is defined in the context of the habitat's features that change, among other reasons, due to human actions.

All of these relations are presented in fig. 4.1.

This scheme shows that the concept of society - defined earlier in this study as the dominant form of sociality - ought to be placed not "over" or "besides" individuals, but rather "amidst" all that which is created as a result of their actions: ones performed at various levels of the hierarchical organization of social life, in a particular habitat transformed by them, directed and legitimized by particular types of social representations shaped by the influence of both the habitat and the collectivity. In other words, society would emerge from relations that link the population, the habitat and social representations.

One essential feature of the model proposed by Linnda Caporael is, therefore, that it obliterates the dichotomy of the individual and society. In her approach, analysis of direct relations (often oppositional) between these two entities is replaced by analysis of interactions between individuals at various levels of 
"sociality." 188 The assumption that each of these levels is nested in lower ones, along with the assumption that functions created at any of the higher levels can be used in the course of interactions occurring at lower levels, eliminate both the danger of becoming reductionist and the danger of "losing sight" of individuals in analyzes of macro systems.

At the same time, however, it might seem that this model achieves this advantageous effect precisely by eliminating society - at least in the sense given to this term in contemporary sociology. It does not entail anything that we have come to associate with the concept of society as a social system: institutions, social structures, apparatuses of power and coercion, economic and educational systems, etc. The place of society understood in this way is taken in Caporael's model by various kinds of base groups. Owing to the functions ascribed to them (the creation of images of reality, the coordination of team efforts and the defining of social identities), they should be simply identified with communities, i.e. as groups comprised by individuals tied with social bonds, which constitute the basic level of social integration and the source of sociality.

This is certainly not an objection against Caporael's model, since it is sufficiently "legitimized," as it makes it possible to explain the dynamics of social life in its particular areas. However, this becomes a fundamental problem if we take this model as a point of departure for explaining social transformations analyzed in terms of types of social organization, i.e. in terms of the transition from the traditional to the modern and postmodern society, even if the existence of the last kind is still hypothetical. ${ }^{189}$ It is therefore paramount to

188 It seems highly probable that the method of analysis proposed here will be accused of neglecting human intentionality, free will, and agency, thus constituting another variant of cultural determinism. Indeed, the discussed scheme does not leave room for the kind of individual agency understood as being "ontologically primary," because the theory of mind assumed here (cf. Chapters Two and Three) argues that individual intentionality is always the outcome of interplay of meanings present in culture (considered as a repository of social memories) and of the use made of those meanings by individuals. However, such an understanding of intentionality has nothing in common with any variant of "cultural determinism" and leaves a lot of room (especially today) for individual decisions stemming from individualized perspectives on reality, one's unique position in the world and the rules that govern that world. As we demonstrate in previous chapters, even if it is necessary for our minds to use cultural contents, both the nature of the main carriers of culture as social memory and the very structure of our brains still leave a lot of space for invention in processing these contents.

189 Contemporary sociology does not question the existence of the traditional and modern order as distinct principles of coordinating actions, although their definition 
consider whether and in what way the concept of society could be included in the discussed model given that "society" is a term referring to historically developed forms of sociality, organizing the functioning of particular communities, institutions and subsystems.

Clearly, this cannot be done simply by assuming that society is a more general variant of community. Regardless of difficulties with cogent arguing in favor of such a claim, and regardless of the fact that it remains unknown what the macrodeme level would be in that case, this assumption leads us back to the starting point of this chapter, namely the problem of the relation between the individual and society. Nor is it a viable solution to assume that society constitutes a kind of a federation of communities; in that case, the concept of community would have to cover extremely diverse forms of social structures (from families to classes and markets); moreover, it would remain uncertain what integrates a social entity defined in this way.

It seems that a much more fruitful path to pursue would be to assume that the above schema (fig. 4.1.) presents - on the historical plane considered here - a model of socializing human actions that is proper to tribal communities, thus representing the simplest instance of this process, which could be further developed along with the increase in internal diversity and intricacy of human collectivities. In tribal communities, we observe a strict overlapping of three qualitatively different kinds of orders that characterize contemporary societies: social, political and economic orders. It is only in the tribal community that teams of men and women, which realized different tasks, as well as families, clans, chieftains, warriors, and shamans, create one institutionalized order bound together by a sense of a collective "we" (social order), taking together decisions that are crucial for the tribe (political order) and jointly utilizing the resources at its disposal (economic order). ${ }^{190}$ Already in chiefdoms - the kind of organization of social life that chronologically follows the tribal community ${ }^{191}$ - we deal with early autonomization

is constantly modified and perceived increasingly often as an idealization and not a literal account of historical processes. The subject of dispute here is whether currently observable processes of change constitute a new kind of social order (perhaps merely prefiguring it) or local variants of the modern order.

190 A sort of a summary of research on contemporary tribal communities, and of analyzes of paleoanthropologists can be found in Diamond (1999; especially Chapter 13). LéviStrauss takes a similar approach (cf. Charbonnier 1969). The latter also emphasizes the role of elaborate ritual systems in eliminating potential conflicts that could prevent reaching agreement in all of these orders.

191 We are referring here to the distinction proposed by Jared Diamond (cf. Diamond 1999, 316-317). 
of the political order since decisions that are crucial for the fate of the entire tribe are made by the chieftain, whose position is heritable. Moreover, at this stage of changes there emerges a close relation between religion and political power since one of the functions of the religious systems was to legitimize the decisions of rulers, who would thus gain influence over the shape of collective representations.

This process of functional autonomization of three basic types of orders gains momentum with the rise of ancient empires, or "agrarian bureaucracies" 192 (a term that better reflects their nature), characterized by centralized power, poorly developed land ownership and an elaborate system of redistribution. The process developed continuously, culminating in the form of organization of social life that characterizes the modern society, where the sustenance and reproduction of each type of order would be guarded by separate social institutions. One could say that each of those types of order concentrates and simultaneously generates specific systems of interaction, which are subordinated to the creation and re-creation of three basic dimensions that jointly constitute a given form of sociality: the social order serves to reproduce the population in its mental dimension, inscribing biological reproduction into a previously developed framework of the psychic apparatus, or of the "social character;" the economic order is responsible for the reproduction of the habitat by transforming some parts of the natural environment into a socialized milieu; and finally, the political order is focused on the reproduction of collective representations, which are the key tool in integrating and mobilizing resources across the entire society in situations of threat or expansion. Therefore, each order "cuts out" a different kind of collectivities in society (cf. fig. 4.2.): the social order - collectivities whose main function is socialization (the family, social group, education system); the economic order - collectivities aiming at production and exchange (companies, banks, markets); and finally, the political order - collectivities engaged in defining the common good (political parties, churches, ideological movements).

Naturally, this division has a purely analytical character. In reality, each domain distinguished in fig 4.2. is connected through numerous links to all others, while particular collectivities may serve functions ascribed to all of the above orders (though to a varying degree). Nevertheless, it should be emphasized that each order also has its unique features and dynamics, which can be analyzed only in

192 The term was suggested to us by Jacek Kochanowicz. 


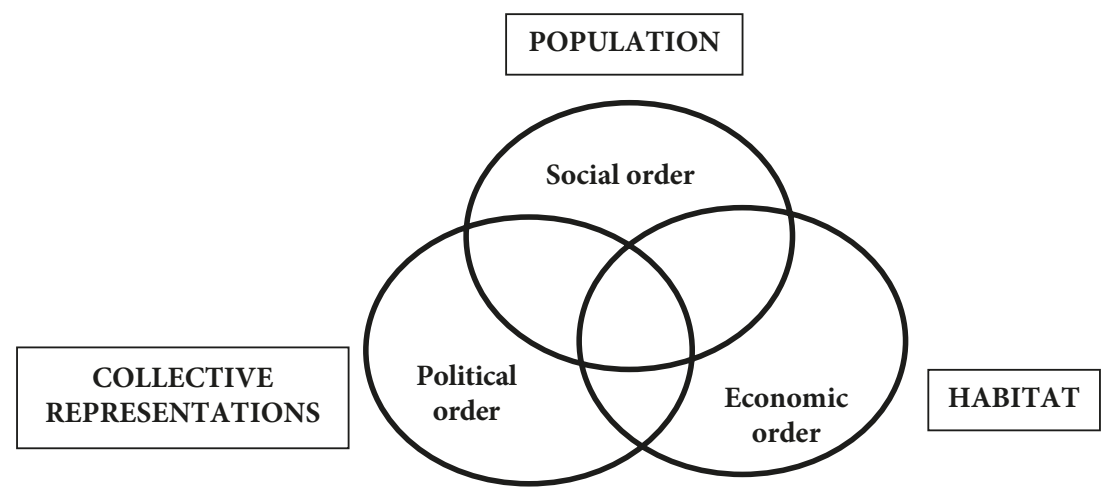

Fig. 4.2: Basic systems of social reproduction

terms appropriate to it. ${ }^{193}$ More importantly though, a given form of sociality (i.e. a given type of society) is, in each separate case, the outcome of interactions which take place not just among individuals - after all, they can belong simultaneously to collectivities that are part of different orders - but rather among institutionalized systems of interaction developed within each particular order.

Accepting this approach to the analysis of changes in types of sociality entails two important consequences.

First, since it follows from the previous chapter that socializing of human actions may occur only on the basis of social bonds, the historical process of functional autonomization of particular systems should be accompanied by the emergence of some forms of communities - let us call them "base" communities - which would not only produce a certain range of social representations, shared by all members of a given system, but also have at their foundation some kind of a social bond. ${ }^{194}$ Therefore, it becomes possible to assume - already at this point, and regardless of the direction in which we shall develop the basic model of socializing processes (cf. fig. 4.1) - that at the foundation of that model we have to find some forms of such base communities.

193 This is what lies at the foundation of economics, political science, and cultural anthropology - disciplines focusing on the analysis of the specific character of particular systems in human societies.

194 One example of such a process is provided by science, as analyzed by Hull (1990) and used by Caporael to illustrate possible applications of her model. Let us also draw attention here to the fact that the process of functional autonomization of particular 
Second, the above description of the three basic types of relations found in this model clearly shows that - by their very nature - they exist in a state of delicate balance. As mentioned earlier, each "reiteration" of a given form of "repeated assembly" is particular and specific - i.e. unique - which means that each may potentially contain elements that not only distort the process of reproduction but also seriously modify the character of these "assemblies." Therefore, the process of multiplying base communities (assumed above) is bound to increase their potential to change to an astonishing degree. It would therefore follow that if a given type of sociality (society) were to acquire a more legible form (not to mention being reproduced), a general idea holding together the entire system would be necessary. It would keep aberrant behavior of individuals within safe limits, both on particular levels of interaction and within each of the distinguished orders. In other words, it is necessary to create a certain kind of meta-community binding all, or at least the majority of members of a given society, by producing a collective "we" and by subordinating their actions to the superior concept of the common good.

The "idea of society" defined in this way is impossible to identify without reference to historical data. The next three chapters of this book are devoted to analyzes of transformations occurring in three areas of social life: the family, work and the public sphere. The choice of these areas was determined primarily by their relation to the three basic orders distinguished above, which are crucial for the functioning of all kinds of societies. However, there was also another reason behind this choice, namely the conviction that each of those areas is specially related to some type of society. The family or, to put it more precisely, clans or houses, constitute the basic unit in the social organization of traditional society. Work or production form the "identity" of the modern society. Finally, the public sphere, or rather problems with negotiating the common good, are the signum temporis of processes related to the transformation of the contemporary, "postmodern" society. Therefore, a more detailed analysis of changes which occur in the course of historical evolution in these three areas could shed light on the very processes of social change, thus helping to identify more general factors that might lie at their foundation.

orders constituting contemporary societies is usually analyzed in sociology in terms of differentiation of social roles. However, the concept of social role reduces the entire problem of sociality to the relationship between groups and individuals socialized by those groups, entirely disregarding the process of the social production of all that comprises a given understanding of roles and related duties. 



\section{CHAPTER FIVE. TRANSFORMATIONS OF THE FAMILY}

Study of the household is the study of community, just as study of the organic cell is the study of biological life.

F. TÖNNIES, Community and Civil Society

Thus the family was buffeted by contradictory forces. On the one hand the roles it was found or assigned to fill accentuated its ubiquity, its strength, and its powers and compelled it to close ranks around its terrible secrets. [...] On the other, increasing awareness of the family's demographic and social role compelled those in powerphilanthropists, physicians, the state - to envelop the family in solicitude, to penetrate its mysteries, and to invade its fortress.

M. PERRot, A. MARTIN-FUGIER, The Actors

The question of the family does not occupy a prominent place in contemporary sociology. This might seem paradoxical since transformations of the family have been widely discussed, especially in recent years. However, these accounts are largely lacking in theoretical depth and generally have a normative character. Indeed, not much have changed in sociological theory of the family since the 1940s when two paradigms emerged: the functionalist one developed by Parsons (1949) and the interactionist one developed by Burgess (1945). The lack of interest in the family amongst sociologists stands in sharp contrast to dynamic developments in this subject in other disciplines. One may even get the impression that sociology surrendered this issue to other social sciences such as anthropology, demography, social policy and economy. Meanwhile, recent knowledge about the family assembled in these fields, especially studies in historical demography, indicate the necessity to fundamentally revise certain claims that are still widespread in sociology, e.g. the conviction about the family's passive role in historical changes, or Le Playa's myth about the "golden age" of the family in pre-industrial times (cf. Szacki 2002, $560-1)$.

Likewise, the question of the family does not occupy a prominent place in sociological empirical research. It tends to appear as a "virtual" mediating 
variable: a vehicle for transmitting social status or inscribing parental values in children. ${ }^{195}$ Its characteristics are usually inferred from studying other phenomena. In studies of the family this entails focusing on individual actors or dyads, chiefly married couples. Still, it would be difficult to recall any example of empirical research on the family understood as a component of the social system - one playing a specific role in reproducing and supporting it.

At the same time, it is important to note that, in sociology, the twilight of theoretical reflection on the family as a specific social institution is accompanied by a strong tendency to proclaim the end of the contemporary family. There are two ways to explain this convergence. First, family would be seen as losing its significance as a sphere of social life, its functions being gradually overtaken by other institutions. That would be the reason why sociology devotes less and less attention to the family, shifting its focus instead to educational systems, social policy, and labor. This is contradicted, however, by the fact that sociologists are inclined to interpret the "disappearance" of the family as one of the crucial factors in the crisis of postmodern society. Certainly, such a thesis contains an implicit assumption that the family is important for the functioning of individuals and society. Furthermore, surveys show that the family is invariably found - both in the most and least developed countries - at the top of the list of "key values in life" (cf. Giza-Poleszczuk 2002). Thus, if the number of divorces or single parents rises, reaching alarming levels, it would mean that people find it increasingly difficult to live a family life rather than that they do not need a family any more.

According to the second explanation, the conviction about the downfall of the family would stem from assuming the kind of a theoretical model of the family that indeed ceased to be dominant in social life. In other words, this more or less openly articulated assumption about the "real" shape of the family would make it impossible to note that it is not family as such that has run its course, but one of its historically developed forms. However, regardless of which explanation is more truthful, both point to the de-theorization of the concept of family. In other words, the idea of family has become banalized, just like the concept of society, which is used almost automatically by sociologists, as we argue in Chapter One.

Both these concepts also share one crucial similarity - in contemporary understanding, both "society" and "family" emerge from ideological and scientific discourses developed in the nineteenth century, which have lent these two

195 This current was started by research on the relationships between upbringing and authoritarian personality, and is continued in research on the so-called parental values (cf. Adorno et al 1950; Kohn 1959; Schooler \& Kohn 1983). 
terms the status of universals. However, as Michelle Perrot and Anne MartinFugier underline, "the family reigned triumphant in the doctrine and rhetoric of all parties from conservative to liberal and even libertarian, which celebrated it as the basic cell of the organic social order. Actually, it was far more chaotic and varied than this triumphal portrait suggests. The nuclear family had only just emerged from broader and still persistent systems of kinship, whose nature varied with locale and setting, social level and cultural tradition" $(1990,97)$.

Already in the 1970s, many scholars (Laslett 1972; Lasch 1977; Levine 1977; cf. also Flandrin 1980) argued that the "family" - understood as a triad comprised of mother, father and children - was initially a chiefly ideological category, while its triumph as the "basic unit of society" coincided (not accidentally) with the period of laying the foundations of democracy and industrial society. In his seminal work, Alexis de Tocqueville observed that "[d]espotism, which in its nature is fearful, sees the most certain guarantee of its own duration in the isolation of men, and it ordinarily puts all its care into isolating them" (2010, 887). "Democracy," in turn, "relaxes social bonds" and "tightens natural bonds" (1031). The family - a natural community, the model of an altruistic "enterprise," and the first school of establishing the collective will - becomes crucial not only to individual happiness but also public good.

All of this means that taking up the question of the transformations of the family requires an immediate bracketing of all associations and contexts, as well as a suspension of the theses that are habitually made in relation to this concept and have taken hold in sociological reflection, appearing as something obvious. We thus need to problematize again the concept of family, assuming that our current perspective on the subject may be in fact particular and historical - that, perhaps, the "ideas of values" that constitute it are elements and expressions of various norms, legitimizations and ideologies, which have made family one of the more important subjects since the beginning of the twentieth century. ${ }^{196}$

\subsection{The universe of the concept of family}

Contrary to popular belief, the concept of family is one of the most complex in sociology. Moreover, in contrast to what common sense suggests, the family is also a radical abstraction. There are several factors that contribute to this.

196 Suffice it to recall eugenics movements and ideologies (cf. Gawin 2003; Weeks 1989), or the harsh criticism of the bourgeois family by Marxists, and Lenin's attack on the family as such. Right-wing ideologies, in turn, have tended to regard the family as the anchor of social order, sanctifying all of its related aspects. 
First of all, the family is essentially a process rather than an entity. It is born when partners form a more or less stable relationship; it grows and expands along with the birth of children; it shrinks as children leave home; finally, it "dies" along with the death of its founders, in the meantime giving life to other families. Let us note here that the process described above implicitly assumes the vision of the contemporary nuclear family. Actually, the process may take a different course. The initial pair does not have to form a stable relationship isolated from its broader surroundings; children do not have to leave home, and if they do, this can happen either earlier or later; finally, the family does not have to die if it is rooted in something else than people. ${ }^{197} \mathrm{~A}$ married couple with children is, in a sense, the culmination of the idea of family: the family accomplished, or the family in dynamic development. At the same time, however, it is only a specific representation of the family, one that does not have to accurately describe its forms found in other cultures or historical periods.

The family is therefore a process also in the sense of its historical duration, extending beyond the horizon of a single generation. When represented by name, heritage or genealogy, it can exist for centuries. In everyday life, the family is a specific stream of events, too: after all, it is realized through a continual recreating of interactional structures - those of the microcosm of home (cleaning and cooking) - or performing household rituals. Thus, the concept of family is only an abstraction: one focused either on a "freeze frame" that briefly captures a mere split second of such a stream of events, or on the synchronic model of the "dominant" process. ${ }^{198}$ The image of a family as a married couple with children constitutes only a specific point in the process of its development, and a normative claim indicating the direction this process is supposed to take. ${ }^{199}$

197 The family could acquire a kind of immortality, despite demographic disasters, in places where it would achieve stability through material representation in the form of a house, and come to be identified with it; where it would not be a particular person but land that receives the nobility's and fiscal privileges; and finally, where people would traditionally "take their name" from the house in the form of "estate name" ("lord over Lule").

198 The "dominant process" is understood here as the kind of a course of family life that prevails in a given historical society. It is usually a statistical entity, a central tendency that shapes processes of forming marriages, as well as patterns of fertility and of dissolution of families.

199 This image became widespread in the eighteenth century, when family paintings became highly popular, no longer showing individual persons but entire groups of relatives (cf. Perrot \& Martin-Fugier 1990). 
Second, the family involves many dimensions that are traditionally studied by different social sciences: biological reproduction (recreating the population), economic reproduction (joint use of natural resources), and social reproduction (continuity of social "units"). Examination of the family as the basic "means" of recreating the population belongs to demography, which considers the family as a reproductive unit. Since Malthus, meticulous records have been kept on the rates of marriages, births inside and outside them, fertility, and women capable of bearing children, because it has become apparent how important demographical phenomena can be for the long-term well-being of societies. Families are, simultaneously, households - specific micro enterprises that operate in the context of the overall economic situation. Control over economic resources has always been a key factor in the functioning of the family, not only for the sake of survival but also due to increasing demands to invest in children. ${ }^{200}$

Finally, families are "political" units, at least in two meanings. In the first sense, family represents the individual's primal space, in which it nevertheless becomes necessary to negotiate the "collective will" (cf. Gauchet 2000). Contrary to the currently dominant vision of the family as a purely positive space free from conflict, the interests of family members do not entirely coincide - neither between the generations, nor between siblings and spouses. Therefore, a legitimized mode of decision-making has to exist in every family. According to Guizot, one of the leading liberal thinkers of the nineteenth century, "[n]owhere else [than in the family] is the right of suffrage more real or extensive. Suffrage comes closest to being universal within the family" (after Perrot \& Martin-Fugier $1990,103)$. In the second sense, it is in the family that the public or common good emerges, understood in the most basic way, i.e. as "family honor," attentiveness and care, or simply the cleanliness of the household. ${ }^{201}$ It is inside the family that there also emerges the category of "common interest," which requires the subordination of individual interests. The clearest manifestations of this would

200 These demands embraced the removal of children from the job market, universal compulsory education and prolongation of the period in which children remain supported solely by parents.

201 As is notable in this context, the concept of "public good" is used today in relation to various kinds of goods produced or managed by the state (defense, public safety, care for the environment). However, according to its definition, the public good is the kind of good that no one can be excluded from - in the same sense as the "cleanliness" of home enjoyed by all of its inhabitants, or the splendor of the family name enjoyed by all people bearing it. 
be sending "surplus" daughters to the convent, or appointing only one heir in the will in order to keep the estate intact.

Although all of these dimensions are regarded today - almost automatically - as coming together in the concept of family, thus employing this term to define a group of related people living under one roof, running a joint household, and using specific modes of taking necessary decisions, "it is [...] important to emphasize that what was referred to in past times as the 'family' was not identical with the father-mother-children triad, and that one cannot study this triad, in the sixteenth, seventeenth and eighteenth centuries without taking into account its relations with lineage or kindred on the one hand, and the domestic staff on the other" (Flandrin 1980, 10). In other words, "being related," "economic community" and "living under one roof" were to some degree separated before the nineteenth century, while categories describing relations between economic reproduction and recreation of the population (and society) oscillated between "lineage," "race," "house" and "family." The combination of the concepts of being related and living together - previously separate things - emerged in the French language only towards the end of the eighteenth century, and more precisely: after the French Revolution, which significantly changed the legal framing of family relations ${ }^{202}$ in accordance with the more general ideas of "freedom" and "brotherhood."

There also exists one more important reason for the difficulties with grasping the concept of family, apart from its processual character and internal complexity. It is the dynamic feedback relation between a family and its broader surroundings. Contrary to the currently widespread vision of the family as a yielding yet passive recipient of changes occurring in the wider social context - at best adapting to subsequent pressures - historians indicate that it is also an active participant in those changes and their facilitator. In an introduction to the famous book titled Reproducing Families. The Political History of English Population History, David Levine, one of the most prominent scholars researching the history of the family, notes: "This essay begins from the premise that the plebeian family was not simply the object of change but that its demographic response to economic forces created conditions which profoundly

202 These changes include the obligation to divide property equally among all children, to allow divorce, and - a true innovation - the necessity to obtain mother's permission to designate a particular man as the father of a child conceived outside wedlock (in other words, even if a man was ready to accept the child as his own, it depended on the woman's consent; naturally, being joined in matrimony would automatically designate the husband as the father). 
influenced subsequent historical developments. [...] The reproduction of the social formation has been situated at the heart of my argument so as to capture one of the most revolutionary features of English history: the silent, cumulative pressure exerted by peasants living on the edge of subsistence, with just a toehold on their ancient rights and liberties. It created the reservoir of labor which was exploited by colonizers and industrializers alike. By focusing on the mentalities and survival strategies which motivated peasant producers (and, of course, reproducers), afflicted as much by social contradictions as their own fertility, we can see a micro motor powering English expansion: first, within the British Isles; next, across the Atlantic; and, third, around the whole globe" (1987, 5 \& 7).

And so we come a full circle: if the family is both subject and object in the process of changes, then it indeed constitutes the basic "cell of social life," in which we can observe the basic processes of "social metabolism" (reproduction of population as well as of social and economic order, and maybe even of the political one). Perhaps, it is due to the more or less precisely formulated intuitions regarding the fundamental character of that "natural community" that the family has always constituted not only an object of idealization, or even ideologization but also a legal, religious, and social institution.

What is more, when political actors, such as state or church, were taking over the right to legitimize marriage and - consequently - children born within it, as well as the right to extend the ban on incest, or to determine the legal rules of inheriting and paternal power, it all had a deeper political sense. As Jacques Mulliez has indicated in his analyzes of the transformations of private life in the Roman Empire, "in its desire to renew both society and state, imperial authorities had to redefine the power wielded by the pater. In this sense, from that time paternity was a political tool in Rome, with patria potestas (paternal authority) constituting an important factor in the organization of the City. It also lasted throughout the Empire as an instrument wielded by state authorities. This could explain the strong influence of the Roman model visible since the sixteenth century when the rise of absolute monarchy based on class society provided favorable conditions" (1995, 46-47). And although the most comprehensive and far-reaching political effect on family was observed only in the nineteenth century ${ }^{203}$ - when "demographic policy" emerged for the first time on state-wide scale, along with the infamous eugenics as one of its key ideas - it is in entire history and anthropology that we find traces of an outside interference with family

203 We have Europe in mind, particularly countries that were "centers of modernization" at the time. 
matters, either in the form of unwritten marriage rules, or family legislation. One could even say that the family has always been a far too important matter to the entire society to leave it in the hands of impulsive individuals.

The problem lies in the fact that this "basic unit of society" clearly cannot be defined, even to some degree, as a stable object with distinct boundaries and a precisely determined set of "functions." ${ }^{204}$ History, ethnology and anthropology have firmly demonstrated a variety of forms, tasks, strategies, sizes, and constitutive principles of that which has come to be traditionally called family. Only by departing from the commonly imposed image of the mother-father-children triad as a "natural community," isolated from its social surroundings by virtue of its privacy, can we begin to grasp the full complexity of this phenomenon, and understand the variety of shapes that the family has taken in the course of history.

Putting aside the family's contemporary representations we shall focus on the processes of which the family is a function, and on contexts in which it materializes in particular forms. "Being a function" refers here not to a determinism that defines "onset conditions," but it rather refers to moments (or periods) of stable harmony in the processes of interactions which take place in the population and in the environment (including the artificial one created in humanity's activities), as well as to interactions between collective representations, in the course of which the concept of a particular social whole crystallizes as family. Regardless of the particular shape that family assumes in a given historical context, in this discussion it shall be regarded as a unit of reproducing the population and its binding structures of social order (cooperation), of economy (acquiring goods), and of politics (power). ${ }^{205}$ This means, at the same time, that reproduction covers not only simple recreation of biological population, but also simultaneous recreation of this population's internal order, and its system of relations with the environment. ${ }^{206}$ After all, family is a "moral entity" that "describes itself, thinks

204 Sociologists invariably enumerate in this context the procreative "function" as well as socializing, expressive, and other functions lost in the course of historical development, namely productive and political ones. In this perspective, it becomes natural to accept the conclusion about the family's twilight - its departure as a social "cell" and the gradual loss of its functions. This invariably suggests either that the social "organism" can manage without family or that it also faces downfall.

205 In reference to the title of David Levine's work quoted above, the basic unit of reproduction is, in this view, a social whole (including family) but not a particular person.

206 The term "recreation" does not mean here a faithful reproduction of the previous state, but rather creation of a new "specimen" or copy - every human being (apart from identical twins, of course) is unique, and - in this sense - every generation is 
of itself, and represents itself as a unified entity maintained by a constant flow of blood, money, sentiments, secrets, and memories" (Perrot \& Martin-Fugier 1990, 131). Let us emphasize this again: this "unified entity" - a unit of reproduction that weaves in itself the three fundamental processes of reproducing social order - does not have to necessarily be an "elementary family"207 - i.e. a triad comprised of mother, father and children.

\subsection{Processes of reproduction}

In line with the model of analyzing social life adopted in this book (and described in the previous chapter), groups created on various levels of interactions are an "interface" through which individuals establish relationships with the natural environment and other people. One has to bear in mind that elements of this "interface" are not just other individuals but primarily various kinds of artefacts containing memories of past experiences earned by a given type of base group with the natural environment (mostly tools), histories of its interactions (language), and its collective self-consciousness (myths, religions, ideologies). Additionally, social life is organized hierarchically: "repeated assemblies" of interactions from higher levels are "nested" in those from lower ones. The "elementary family" (a unit "assembled" around the task of biological reproduction) can be treated as the lowest level of the above hierarchical organization and the groups it creates: a level resting on dyadic "micro coordinations," primarily between mother and child, as well as between mother and father ${ }^{208}$ or among other pairs. Regardless of the whole configuration's stability, the condition for purely biological reproduction is the repeated character of interactions between woman and man, and between woman and child.

This does not mean, however, that this kind of an isolated unit of reproduction would be indeed the basic "cell" in the hierarchy of the social organization

different from the previous one, just like every configuration of social or economic order. The very process of "replication" carries the potential to change, especially if the environment changes too.

207 From this point on, we shall use the term "elementary family" to denote this fundamental triad, a somewhat minimal group necessary for reproduction to occur. Although the term "nuclear family" carries the same intuitions, it is used in sociology in very specific contexts. By adopting a new term, we wish to avoid evoking habitual associations with those concepts of family that currently prevail in sociology.

208 Regardless of the extent to which their relationship is stable or institutionally sanctioned. 
of reproduction, while the "triad" would be - within this unit - a distinguished subsystem of interactions. The composition and size of basic units of social reproduction can vary depending on the context, primarily on the historical conditions in which the process unfolds. Therefore, besides the term "elementary family" it becomes necessary to introduce the term "family unit" understood as a task team crystallized around the recreation not only of its own form but also that of the larger social entity. "Family unit" can be thus considered as a specific group focusing on the realization of a common goal; ${ }^{209}$ moreover, both the goal itself and the boundaries of this group are parts of a "shared reality" that is characteristic for a given type of society. ${ }^{210}$

It needs to be remembered though that population, habitat, and collective representations comprising a given form of sociality are dynamic "objects" generated through continuous processes of creation and recreation. Population is produced and reproduced in the purely biological sense; the environment - in the process of labor; ${ }^{211}$ finally, representations - in the process of communication. At the same time, each of those processes generates specific structures that assemble interactions occurring in collectivities engaged in those processes: biological reproduction - structures of relations; economic reproduction - structures of co-dependence in the process of production; and finally, the reproduction of communication - systems organizing the shared world (common knowledge). These realms may overlap or coincide to a different degree: in tribal societies, genealogical myths would tie the entire population in a web of kinship (cf. Ossowski 1966), while in contemporary societies they define only its small part.

The elementary family, one that prevails today, does not necessarily have to be the place or "cell" in which "streams" of goods, symbols and genealogies meet. The family unit also can assume numerous shapes, comprising a variety of realms

209 We pass over the question of defining this shared goal here because it certainly changes in history. It has surely been related to continuation (i.e. has a long-lasting character), either in the sense of merely surviving, or winning fame and even immortality.

210 In this sense, the representation of family as a specific social relation is bound to be enmeshed with a broader vision of the world created at the level of the base group. The family "sees itself" only from the perspective of a larger social entity; this is one of the reasons why family has always been involved in collective self-perception, or has been incorporated as part of more general policies. In the theory of Michel Foucault (1988-1990) this means that family is invariably involved with the discourse of power.

211 Labor is understood here as a specific system of interactions with the environment, as a result of which the latter becomes transformed, thus becoming the "object" of subsequent reproductions. 
and privileging some over others. This is because the tasks faced by this unit are strictly related to its context, i.e. to demographic, economic and social trends.

Considering the family unit as a "meeting space," where larger systems are bound together and launched into the future through children, entails several crucial consequences.

First, when understood in this way, the family can include inside itself a lesser or greater number of people - not only relatives but also servants, slaves or liegemen. ${ }^{212}$ Second, it can function as a more or less self-reliant, isolated unit - all depending on the strength and character of relations that tie it to its surroundings. Third, it can be oriented towards various tasks: either primarily towards securing the "production of people" or towards "economic expansion," along with continuity in re-producing resources, or towards guaranteeing "social expansion" by extending the web of bonds and building a social position. And finally, fourth, the family unit has a clearly dual character: it is a whole for those who belong to it, but at the same time it remains part of a larger system. The same applies to its members: they belong to families, but can also be part of other social configuration: clans, work teams, religious sects, or social classes. Thus, depending on what part of activities engaged in by members of the family unit takes place inside and what part outside it, and on how their loyalties are shaped, the family can be emptied or filled, gain or lose significance, both for its members and for other social configurations.

Here, in this "silent, cumulative pressure exerted by peasants living on the edge of subsistence," in the marital calculations of aristocrats, in the hard work of a Puritan father of the family, hides the revolutionary power mentioned by Levine - one that changes history. Social order is created and recreated by family units and other social configurations, which permeate one another. In its various manifestations, the family unit always constitutes a vehicle for the reproduction of both physical people and larger social, economic and demographic entities. Just as the married couple is a "mechanism of generating genetic variety" - after all, each person is different and everyone constitutes a unique combination of

212 This phenomenon is captured by the well-known concept of "extended family." Although Le Play was mistaken to assume that in its golden age, i.e. before the Industrial Revolution, family in its "extended" form united (in one household) three generations and cousins twice-removed, he was right about the fact that family before the Revolution differed from the so-called nuclear one. However, it is important to remember that this family would incorporate dependent people or servants rather than blood relatives, while its size was strictly correlated with social status: the higher the status, the bigger the family (cf. Flandrin 1980; Levine 1977; Laslett 1972). 
characteristics inherited from parents - so it also performs that function in relation to the other two processes of reproduction (i.e. social and economic ones) because no child is brought up in exactly the same environment, and every product of labor is unique.

In this sense, the family unit has an abstract and temporary character: it is a dual structure shaped at the boundary between past and future, as well as at the point where various social configurations meet. The family contains within itself - albeit in a hidden fashion - both hallmarks of continuity and harbingers of change. This is precisely what makes its essence so difficult to grasp. ${ }^{213}$ The family is always local and the only thing that could be indicated as a regularity in its development are tendencies related to clearly distinguished features of the context. $^{214}$

The major challenge in speaking about family, especially about the transformations of its character, consists in identifying the factors that decide about the shape the family unit takes, about its ties and relations with the surroundings, and about its internal character. The knowledge we have today on the prehistory and history of family suffers from two important limitations. First, it is gathered from a specific perspective - e.g. anthropological research of the Boas school was conducted in the context of criticism of American middle-class families. Second, that knowledge relates to only a specific kind of family: in traditional societies, these would be mainly families from the upper levels of social structure, because only they left the sources we can study now; in modern societies, these would be middle class families because they were the gravitational center of the new order; finally, in contemporary societies these would be "sick," broken, pathological families from the lower social tiers since they are the focus of social policy.

It would be difficult to present, in a comprehensive manner, historical family configurations in all their richness and variety. In this review, we shall focus on

213 Historians keep pointing out difficulties related to attempts at grasping regularities in the "development" of family. In the same country, at the same point in history, a family of peasants from the Paris Basin fundamentally differs from families of Breton peasants, just like there is a difference between English plebeian families living in adjacent counties. That is why "family" - when extracted from the complex web of relations and dynamic processes - constitutes an abstraction. In other words, family as such does not exist - there are only its representations, either produced at the level of social self-consciousness, or through science, politics and ideology.

214 These would be "relational linkages," to employ the terminology proposed in the previous chapter. 
those that constitute clear and distinct types: clan-like families in primal societies, large family structures in feudalism, the full family of the blossoming middle class, and finally the shaky, broken family of the postmodern era. ${ }^{215}$

\subsubsection{Family constellations: societies without history}

A review of anthropological data and studies on societies "without history" mostly hunter-gatherer ones - leads to the conclusion that they had a genuine obsession with blood relations as the basic structure that determines relationships within the group. It is equally striking to note how a relatively slight importance is attached to the elementary family, i.e. married couple with children. It seems to have the status of a "dependent unit." In some tribes, like the Nayar, who live at the Malabar coast, marriage would be established symbolically, with no lasting ties linking spouses, and real authority over woman and children would be wielded by brothers-in-law. In other communities - like the Massai or the Chaga from Africa - the marriage-based family is only one of many existing forms of relationships; in other tribes, wives could be borrowed. The dependence of the elementary family also manifests in the practice of often arbitrary assigning of kinship to children by either tying them only to the father's group, or solely to the mother's.

This "dependent" elementary unit still plays a vital function insofar as it provides the group (clan, tribe) with new people. Among some tribes, like the Brazilian Indians Cashinahua, a relationship is legalized as marriage only when the woman becomes pregnant. Fertility is one of the dominant subjects of rites, magic and art. ${ }^{216}$ In such a configuration, the couple "produces children" not for itself but for its (partner's) group, which further makes it a medium (tool)

215 Roman family and the lineages of ancient Greece roughly approximate the forms found in European feudalism. Hence, we do not present them as a separate configuration. While discussing the construction of Greek family, Carola Reinsberg writes: "The fact that in Homeric times marriage constituted a universally accepted form of life stemmed from the system of organization of that society. It was an aristocratic and feudal system based on the political and economic power of particular houses, whose continuity and survival were guaranteed precisely by the institution of marriage as a form of managing the oikos (household), both in the narrow and broader senses, and by securing rightful heirs" $(1998,15)$.

216 Primitive figurines typically represent women with excessive bodily features emphasizing the symbolism of fertility. In ancient Greece, both maidens and married women would still visit the temples of Priapus to enhance their capacity for bearing children (cf. Reinsberg 1998). 
of reproduction rather than an autonomous social unit. One could argue - following Lévi-Strauss (1985) - that a reversal of roles can be observed here: it is not marriage that gives rise to family, constituting its foundational act, but rather the family brings marriage to life. It is the main and socially sanctioned means, at the families' disposal, of surviving and establishing relationships.

In tribal societies, marriage also does not constitute a key element of social structure; it is rather considered a tool for strengthening the community by forming unions and alliances. A skillful handling of "marriage selection" often allows to gain support in struggles with enemies, or obtain information about fertile areas. This in turn significantly consolidates the group. ${ }^{217}$ It also happens, as Gilmore shows (2001), that spouses do not live together - both the forming and un-forming of the relationship takes place without larger ceremonies, while responsibility for the child is taken by the uncle, not the father (avunculate), and children are distributed to clans in accordance with their parents' sex (boys to the father's clan, and girls to the mother's). After the period of breastfeeding, when children are constantly with the mother, they are moved to a group that rears children collectively, and then - in the case of boys - are initiated and moved to a group of men. In this sense, the child neither belongs to the married couple nor is only their responsibility - children are rather a kind of "public good."

Scholars researching primal societies were also struck by a fairly far-reaching sexual freedom, especially in contrast to their own, bourgeois customs. This image of a primary community contributed to an idyllic and plainly false vision of a non-oppressive community or even "primordial communism" in which there is neither private property nor the family. ${ }^{218}$

Let us investigate in detail the features of the context in which blood relations gain primacy over elementary family ${ }^{219}$ and in which fertility is emphasized as its

217 One of the testimonies to the precedence of group interest is the ubiquity of systems of forming marriages, which define in strict terms the rules of making pairs, in line with more general principles of "weaving" the population and reproducing it.

218 As far as the idyllic character is concerned, a special role was played in its spreading by the culturalist school of Franz Boas in USA, primarily through Margaret Mead's research on Samoa. As to the "primordial communism," it was Friedrich Engels who observed - in The Origin of the Family, Private Property and the State (1884) - that family is a derivative of private property, which has allowed relations of power and domination to enter into the realm of "natural" relations (based on equality and partnership) between man and woman.

219 It is important to stress here that the married couple is not related but establishes kinship between families through children who are related to the families of both spouses. 
major task. In the realm of demography, primary societies are characterized by a fragile equilibrium stemming both from short life expectancy ${ }^{220}$ and restrictions imposed by the habitat's capacity to provide food, requirements for mobility (movement in the case of a nomadic lifestyle) and defenses. The size of the population and its structure become in this situation one of two key conditions for survival, apart from natural resources. The collectivity thus needs to balance two contradictory pressures - on the one hand, limited means of living (the pressure to constrain its size), and on the other: efficiency in economic, reproductive and military terms (the pressure to increase its size). Hence the cult of fertility is accompanied by the admissibility to commit infanticide, practiced in relation to children born "untimely."221 In this way, the population can remain at a sufficiently low level so as to facilitate direct contact among group members. The environment, from which resources are obtained, is only slightly transformed. It is exploited until natural resources are exhausted, whereas "tamed" or "cultivated" only to a narrow degree. Moreover, an appropriated environment (one that allows itself to be exploited) constitutes the group's common good - even meat from hunted animals would be distributed among all members of the community. ${ }^{222}$

A high level of risk causes life practices to become extremely rigid: the fragility of an existence that balances on the verge of survival makes any experiment a threat to the group's life. Internal conflict is a danger too - hence the vast importance of integrating rituals and the emphasis on the necessity to follow tradition closely. The significance of the population's structure - i.e. the right proportion of adults to children, and women to men - is linked to the fact that the survival of the entire collectivity demands an adequate, full "cast" of all task teams (hunters, gatherers, children, and the elderly, who embody collective memory). In this context, the basic "unit of survival" is not the elementary family but the larger "family unit" (clan) or even the entire tribe.

In this sense, the married couple is only a "vehicle" for weaving together family groups through a community of blood embodied by children.

220 This makes elementary family an ephemeral construct threatened by the premature death of any partner, who then needs to be replaced; this is yet another factor contributing to its "dependence."

221 "Untimely" means here the situation when the woman is already burdened with one small child and the tribe needs to move; when twins are born; when the woman is already breastfeeding one child and there is risk she might not be able to feed both; and finally, when the mother dies in labor (cf. Daly \& Wilson 1988).

222 However, the prestige of a good hunter remains an individual asset (cf. Diamond 1997). 
There can be no doubt that due to its size and lack of intense contacts with the outside world the tribe is a strongly endogamic population. Kinship is therefore an actual factor in structuring the group, imposing on it a "metric" of closeness and distance. Little wonder then that collective representations arise exactly from the concept of kinship: the vehicle of community, the guarantor of solidarity, and a factor structuring the group. Totemism, cult of the ancestors, and genealogical myths ${ }^{223}$ make the idea of kinship cover also the past and the natural environment.

Within this system, there is no place for a singled-out position of elementary family: the collectivity is rooted in clans, groups of men (hunters, warriors) and women, as well as the tribe's elderly and shamans. The tribal community is a reality in which the realms of biological, social and economic reproduction almost overlap, with the major role played by the reproduction of the population as the most valuable yet simultaneously fragile resource.

\subsubsection{Lineage, race, and house - constellations of the "traditional" family}

There can be no doubt that in traditional societies and communities the family unit becomes the basis of social organization. Nevertheless, this unit is still not what we understand as family in today's sense. In a historical analysis of this concept, Jean-Louis Flandrin (1980) indicates several models between which feudal Europe oscillated: "lineage," "race," "house," and sometimes also "family," although the last term was used - quite characteristically - in relation to the lower classes. Elementary family - a married couple with children and with a visibly dominant father - was still a dependent unit embedded in an overarching social whole.

The key issue for grasping the constellation of family life in a traditional society is to recognize the role and demands of the land as the basic economic resource. Except for communal lands in some European countries, land was privatized. It was all the more valuable when there were fewer opportunities for expansion through deforestation or fertilization of wastelands. Land becomes the center around which the reproduction of the population is organized: in order to participate in the stream of reproduction one needed to be "settled." This applies both to aristocratic or noble families and peasant ones. ${ }^{224}$

223 It is worth mentioning at this point that the spirits of the ancestors and genealogies create the past as a factor that plays an active role in the present.

224 This did not concern the families of craftsmen and tradesmen; except for these groups, however, land was a key resource for the entire population. 
As an attribute of social strength, however, land imposes its own limitations and demands on the reproduction policy. On the one hand, land needs an heir, but on the other it could not have too many of them because that would pose the risk of fragmenting the estate, which could lead to the diminishing of the family's social position. Hence, most Western European countries would employ the principle of entail, i.e. the transferring of the estate intact to just one heir, most preferably to the eldest son. And since the possession of land was the condition of engaging in reproduction, a large number of high-born children would not enter matrimony. This particularly affected women, for whom there were no alternative career opportunities in the army, allowing, for example, to enrich oneself during wars. As a result, the mediaeval matrimonial market was characterized by male hypergamy: high-born maidens unable to inherit outnumbered equal but inheriting sons. This allowed lower-born young men to enter the elites (Duby 1997-1998). The necessity to wait for the passing over of the estate greatly extended the period before marriage, especially among men - heads of noble houses were reluctant to concede power to their sons.

Putting biological reproduction into the service of economic transmission or expansion caused marriage to be determined not by love but rather by larger goals of the family as a whole. Still, it would be expected of marriage to lead to the "production" of an heir. Apart from incest, infertility and the non consummatum clause were the only permissible reasons for annulling the sacrament of marriage. The subordination of the family unit to the demands of the land caused - as Bourdieu aptly put it in relation to affluent peasant families from Brittany (1972) - that it was not the son who inherited the land but rather the land that inherited the son.

A similar tangle of factors characterized - as Witold Kula has accurately demonstrated (1983) - peasant families in Poland's eighteenth-century serfdombased economy. A peasant marriage was subordinated to the demands and needs of the farm. The family was a unit of production; more than that - the mode of production would determine family's optimal form. The efficient functioning of both the family (securing means necessary for survival) and the farm (optimizing productivity) required meeting specific conditions. First of all, a peasant family would have to be full: the farm needed both men's and women's work in a strictly defined proportion. That is why richer families would include farmhands and servants, while widowed parents - if their children were not mature enough to provide the farm with adequate workforce - had to remarry under threat of losing the farm or being transferred to a smaller area (one demanding less work). Landowners holding all rights to the land had the prerogative of making such transfers; sometimes it was even the landowner's duty to find a partner for the 
widowed peasant. Given high mortality rates, especially among women in labor, this immensely complicated the structures of peasant families. It was quite commonplace to see families where children from the current marriage would live together with ones from previous relationships of either husband or wife.

The requirements of the farm also dictated the "optimum" number of children. Child labor played an important role on farms and constituted the only way of securing provisions for elderly and decrepit parents given the obvious lack of today's pensions. According to one Polish proverb, which has survived to this day, "children are the peasant's treasure." Due to the brittle balance of the relation between "mouths and farmlands," an exact right number of children had to be secured. If there were too many children, this could spark problems with feeding them. If there were too few, managing the farm could become problematic. However, the right number was difficult to achieve in the face of high mortality rates. ${ }^{225}$ This caused repeated remarrying and "redistribution" of children to be commonplace phenomena on eighteenth-century Polish farms. The practice of transferring (not adopting) "useless" children to other parents was widespread; such redistribution simply constituted a legal prerogative of the manor's owner.

The demographic factor played an immensely important role in the economy of the traditional society, both due to the risk entailed by high mortality rates and unexpected deaths, which endangered the farm's efficiency in working the land, and in relation to the surplus of people, which could not be easily resolved given the overall low efficiency of agriculture. This kind of society was characterized by a very slow growth of population, which stemmed mainly from numerous demographic catastrophes (due to disease or hunger) and low average life expectancy resulting chiefly from high mortality rates among children. Reproducing the population in the face of depopulation comprised one of the crucial concerns among rulers in relation to their subjects, and among subjects themselves, mainly in the case of great houses. Rules that forbade migrating and ensured an endogamic system of marriage - which were widespread in the Middle Ages, when "marrying out" from the village was frowned upon - constitute a testimony to efforts made in order to secure demographic balance. The importance of fertility - even after Christianity was reinforced ${ }^{226}$ - can be clearly discerned

225 According to certain estimates, some two-thirds of all children would die in mediaeval Europe (cf. Chaunu 1971).

226 Christianity delegalized numerous pagan fertility cults. 
in the above-mentioned sole reasons that the Church saw as valid ground for divorce: infertility or non consumatum.

What structured the population was no longer kinship as a general idea of the social whole, but the endogamic concept of the purity of blood, which manifested in the concept of the "estate."227 This was related to the immense growth in the number of people - in comparison to tribal organization - who were then "consolidated" in more or less violently created territorial wholes: countries, counties, and fiefdoms. The community of genealogy, understood as descending from a single common ancestor, was impossible to uphold as a basis of such consolidation, just like it was impossible to derive society's internal differentiation from the life of the mythical ancestor. ${ }^{228}$ As a new social idea (representation), the estate retained primacy over family units, constituting a base group for them (deme), i.e. one in which processes of reproduction could take place. ${ }^{229}$ The estate involved not only marital endogamy but also a cultural one - with regard to the mother tongue, customs, lifestyles and knowledge - and an economic one, which entailed principally the ban on labor for representatives of the higher estate. ${ }^{230}$

The importance to preserve "distinctions," along with requirements stemming from land being a crucial economic resource, comprised an additional

227 In the above-mentioned work by Stanisław Ossowski, titled Więź społeczna a dziedzictwo krwi (1966), the author demonstrates how genealogical myths (deriving the collective from one common ancestor of the entire house) were replaced with Bible-derived myths about many ancestors ("race"). In Poland, the nobility was said to derive from Seth, while peasants - from Ham.

228 Particular events from the life of the ancestor - e.g. meeting a kangaroo, or crossing a river - would often become mythical moments of establishing clans (of "Kangaroo," of "Frog" etc.).

229 However, it is worth to point out here one crucial modification: in biology, the boundaries of deme are determined by spatial factors (the criterion of physical access), which still played an important role in tribal communities, although they were also supplemented with culture-based criteria. In the feudal society, it was the latter that acquired a key significance, which is clearly visible in the case of powerful houses whose members would sometimes even marry people from the other side of the continent.

230 Labor was a plebeian preoccupation; in continental Europe, the nobility was not allowed to engage in trade or industry. The only admissible posts were in the clergy, in the army, at the court, or would involve holding offices, which were often inherited at the time as a sinecure. It would be difficult to deem any of those preoccupations strictly as labor, which we shall return to in the next chapter. 
factor strengthening control over marriages, now treated not only as a tool for preserving the status quo but also as a means of developing the power of noble houses. As Jack Goody attempts to demonstrate (1983), both royal and church authorities adopted various strategies in order to limit the growth of the power of noble houses. For example, extending the ban on incest was not only a source of revenue from dispensation, but also made it more difficult to accumulate wealth. After all, in aristocratic families everyone would be somehow related, especially if one used the Germanic method of establishing kinship. ${ }^{231}$

Reproduction - and the elementary family along with it - were thus subordinated not only to the logic of controlling land, but also to social and political games, in which the estate emerged as a unit superior to lineages, families or houses. Elements crucial for reproduction - marriage and status of the child were brought under the regulation of written law executed by state institutions. This meant that collective representations of family were incorporated into the system of reproducing the political order itself. Christianity (and its kin monarchy) favored "legitimate" families based on "rightful marriage," or ones able to approximate that image. It seems natural to discern here the influence of Christian morality, but it is not the only element motivating this inclination. The elevation of marriage, which became a sacrament ${ }^{232}$ and began to play the role of "social police," was a very useful tool in the hands of monarchy, which desired to secure a social order in which everyone would occupy the right place.

This was the beginning of the ceaseless interference of church and lay authorities with the organization of "family." A "rightfully married" man could not be a father the way he liked, while children conceived out of wedlock were deprived of any rights. The father was not the one holding power in the household but one indicated as such through legal marriage, or - to quote the wellknown words of St. Paul - pater is est, quem iustae nuptiae demonstrant. One

231 According to this method, given the ban on marriages within seven degrees of consanguinity (which equaled as many as fourteen degrees when calculating with the Roman method), a man ready to marry would be prohibited from coupling with as many as 2,731 cousins from his generation (assuming an average demographic trend), which practically meant all marriage-ready maidens he could meet, or even exceeded their total number (cf. Flandrin 1980).

232 It is worth noting that the resulting indissolubility of marriage ("till death us do apart") greatly stabilizes the family unit and guarantees that its property is not fragmented (cf. Fisher 1994). 
who used to be the head of the family due to certain individual characteristics could now obtain such status only by entering marriage, and could become father only to a child conceived with his legal wife. If the pater accepted such limitations with respect to the legitimacy of the relationship and children, this was only because he was more than amply compensated with legal and religious guarantees of power in the household, a power extending to the public sphere through fiefdom.

In ideological and cultural systems of the traditional society, the family occupies a central place. After all, at the heart of Catholicism we find the Holy Family, which constitutes, as it were, a model for all earthly families. However, the full realization of the idea of family as a primarily spiritual, and not a biological, unit (after all, Joseph was not the biological father to Jesus) was postponed until Reformation, when Protestantism put the father on a spiritual pedestal (Bogucka 1998). Since the reforms of Gregory VII in the eleventh century, marriage was declared a sacrament despite its ambiguous sense in the New Testament. ${ }^{233}$ Thus, it was deemed the only legitimate model of reproduction. The period also saw the first humble attempts at interfering with family's "internal" matters such as the authority of parents over children, relations between spouses, and bedroom secrets. However, the legal system's focus was on financial questions, mainly inheritance, the right to the woman's dowry, and matters of marital arrangements. Both the king and the Church found the noble houses to be of crucial importance - both as an ally and as a competitor in the fight for power.

These new references constituting the dynamic of family constellations hide behind the above-mentioned variety of terms referring to family. Lineage is a term used in reference to the genealogical continuity derived from a pair of founders, incorporating all members, both in main and lateral lines. Race in turn refers to the continuity of the masculine ${ }^{234}$ name, which was achieved through male heirs, although it would be often "patched" by adopting closer relatives. This term is strictly related to the concept of pater familias as the creator of a family-race. House would mean land whose inheriting established a chain of continuity, although not necessarily that of a "lineage" or "race." When there was no male heir, the husband of the daughter could adopt the name of the "house," thus "patching" the demographic gap in the continuity of the bloodline.

233 According to St. Paul, marriage constituted a lesser evil than unregulated sexual life, but celibacy was still the desired ideal.

234 Here, in the privileging of the masculine genealogical line, we find the legally guaranteed dominance of the father-patriarch in the family unit. 
All three concepts refer to the longue durée, establishing social categories from a level higher than that of elementary family. The married couple and its children would be the moment of transmitting "house" or "lineage" into the future, its expansion in time and space, rather than an independent social unit. That is why the term "family" is mainly used in reference to the lower classes who had no "extension" in genealogy or land and were, by the same token, sentenced to a short life deprived of any significance. ${ }^{235}$ What is more, wealthy peasants or artisans would use the concept of house rather than family (Flandrin 1998). Relationships between spouses, parents and children were also subordinated to the logic of long lasting, which dominated over that of emotions. As Michelle Perrot and Anne Martin-Fugier point out, "all societies - except for ours, where mediating structures have disappeared, leaving the state and the married couple, as it were, facing each other alone - expressed distrust for exaggerated love between spouses because it could make them forget about their duties to the society" $(1990,198)$. A similar severity characterized the normative ideal of parents, especially the father. The propagated principle was: "the more a father loves his child, the more diligently he teaches it and punishes it," or - following the teachings of the Ecclesiastes, who was often mentioned in sermons - "one who loves his son often uses the cane" (Delumeau \& Roche 1995).

Therefore, depending on whether emphasis would be laid on the continuity of name, genealogy or property, the family unit would be defined in various terms, which then became mixed in use. However, it has always been a unit extending beyond the elementary family, incorporating in itself - as a point of reference vast structures of kinship, numerous unrelated household members, and also covering (through the extended metaphor of the father) the clientage of the house. "Family," in the sense closer to the elementary family, can be found in the language used to refer to urban lower classes or the countryside poor - people who had nothing to transfer, who were barely able to survive and therefore focused primarily on the purely biological task of physical reproduction. This is a significant observation that indicates presentist intuitions behind the concept of family as a unit without past, one that functions on the margins of economic processes and social reproduction, providing the population only with "naked" people. These intuitions shall find their development in the modern industrial society.

235 Significantly, as distinct from families from the higher classes, ones from lower tiers were easily fragmented, losing their continuity. Thus, they are not the long-lasting chains of names, properties or social positions (noble titles). 


\subsection{3 "Family" as a vehicle for the reproduction of social structure: the dominance of the logic of market and state}

It is in the modern industrial society that elementary family emerges as a clearly distinguished basic social unit, one that is problematized in public discourse. Larger units - e.g. lineage, house or race - are no longer the place where the three dimensions of social reproduction are woven together. It is the "rightful" nuclear family: the household, the space of living together, "natural community," and the original space of democracy. This representation certainly does not reflect the complex reality of family life, but rather suggests or even creates it: "family - society's 'invisible hand' and the economy's 'hidden God' - was at times a conspirator within the very heart of democracy; it straddled the ambiguous boundary between public and private" (Perrot \& Martin-Fugier 1990, 125).

The shape of the family unit began to change, although it needs to be remembered that the shift from agrarian to industrial economy was a slow process in European societies. The significance of land as "capital" or the resource conditioning social position gradually began to wane as financial and "human" capital came to the fore. The emergence of industry, and the job market along with it, allowed young people to seek economic independence away from their original family unit; work and skills became a resource that could be sold.

However, it would be entirely unjustified to regard the family unit as a passive recipient of changes occurring independently from it. Indeed, it played a crucial role in shaping modern industrial society. John Hajnal (1965) indicates a specific form of Northern and Western European family, which - he argues - was one of the factors behind the emergence of the new economic model. Principles of inheritance returned non-inheriting, well-educated noble sons to satisfy the needs of growing trade and industry, especially in places where estate rules did not prevent them from taking up work.

At the dawn of the transition to modernity, traditional society's possibilities of expansion were exhausted - land was already distributed, while forests and swamps were robbed of all patches that could be cultivated; in England, enclosures cut at the roots of the existence of district communities. At the same time, Europe was showing first signs of the "Vital Revolution" that was to come soon (Chaunu 1971): children began to live longer thanks to better conditions and increased parental care. The number of unmarried individuals among the higher classes also rose as a result of blocking the possibility to "settle" on one's own land. The emergence of new areas of economic activity - or rather, an active 
search for them ${ }^{236}$ - paved the way before the non-inheriting to independently create their own economic basis for reproduction.

The strengthening of the nation state, which began with "enlightened absolutism," was simultaneously a process of wearing away the power of houses. One consequence of the expansion of the state was also the increasing legal interference with things that previously were decided solely by the family unit, primarily by the patriarch. Commenting on events sparked by the French Revolution, Andre Cabantous observes: "Deep economic, social, political and cultural changes that transformed the French society in the years 1750-1920 also affected the father figure, differentiating its function and image. From that point on, apart from the pater familias who rules over his sons, there slowly emerges a migrating or absent father, a divorcee" $(1995,323)$. Here is a list of blows dealt to French patriarchy in this period:

1792 - women gain the right to file for divorce

1793 - wealth has to be distributed equally among children (ban on free allocation of wealth in the will)

1841 - children forbidden to work in manufactures

1874 - child beggars act (parents made responsible and punishable)

1874 - Roussel Act (the right to inspect - by state officials, naturally - the well-being of children handed over to wet nurses)

1889 - paternal rights can be stripped in favor of social care if father is deemed

"unworthy"

1898 - act on mistreatment of children

1912 - establishing fatherhood becomes women's right

1935 - abolishing of the right to punish children physically

It is not hard to discern that an absolute majority of the above legal acts concern children. On the one hand, they aim to secure children's well-being. Importantly, the child does not belong solely to its close ones, but is "future of the nation and the race, tomorrow's producer and progenitor, citizen and soldier. Between the child and the family - especially the poor family, presumed incapable of caring for its own - a host of third parties intervened: philanthropists, physicians, and statesmen determined to protect, educate, and discipline the young" (Perrot \& Martin-Fugier 1990, 196). On the other hand, these "third parties" support children in their conflicts with father and family home, providing them with a strong

236 These possibilities did not emerge on their own - they were the effect of an intensified search undertaken in the face of rising economic pressures. 
institutional ally in family's internal disputes. ${ }^{237}$ However, regardless of whether revolutionaries really turned sons against their fathers, the occurring changes legitimized and made it easier for young people to escape from the supervision of their parents, strengthening at the same time the tendency to seek economic independence.

The rise of industry, especially in the first phase dominated by outwork, opened new possibilities of becoming independent also before peasant children. As David Levine has demonstrated (1977), the chance to obtain means to achieve self-reliance led to the lowering of the marriage age, thus increasing their number, and - in consequence - causing a demographic boom, which gave rise to scientific demography ${ }^{238}$ and made matters of population an object of interest to the state. However, earning economic independence also had its other side: Levine writes in this context about the loss of control over conditions of reproduction (1987). Land was and still is a stable, at least partially predictable resource of means, one that allows long-term "planning." ${ }^{239}$ At the same time, the job market - especially in the early stages of industrial growth - was capricious and unpredictable. The plague of unemployment described by Engels in The Condition of the Working Class in England (1844) deprived masses of workingclass families of all means, although this was, in turn, caused by the surplus of available workers following the demographic boom rooted, after all, in the possibility to earn a living in the industry. ${ }^{240}$ This is one of the most convincing examples of feedback between economy and demography.

Demographic and economic factors supported and accelerated each other. It was not "industrialization" that forced families to adapt. Rather, changes in strategies of forming family units, caused by the closing off of the environment

237 It needs to be acknowledged that this kind of support was used not only by children but also by parents. At the height of the family's triumph, people like "insane and feeble-minded had no rights as citizens. Under the law of 1838 they could be held on request of their families. [...] Confinement of 'madwomen' increased dramatically over the course of the nineteenth century: from 9,930 in 1845-1849 the number of female lunatics rose to 20,000 in 1871 [...]. In 80 per cent of the cases these women were committed at the behest of men (a third of them husbands, the rest fathers and employers)" (Perrot \& Martin-Fugier 1990, 170; emphasis added).

238 We mean here the theory of Malthus.

239 Surely, crop failures could occur but in the long run one could count on the land's ability to "feed" its owner.

240 The second factor in the plague of unemployment was the shift from outwork to factory work; in the case of the latter, efficiency caused a drop in the demand for workforce. 
due to the shortage of land and a rapid growth in population, were - as Levine argues - "the silent revolutionary force" that changed history.

This does not entail the loss of significance of the family unit, but rather its reorientation towards a new dimension, namely the moral one. Since the French Revolution, "family" has been one of the fundamental themes in ideologies, both conservative and liberal. According to Hegel's Elements of the Philosophy of Right published in the 1820s, individual is the subject of law (law can be only personal); however, this does not mean that this individual is socially autonomous. He or she would be rather subordinated to the civic society, of which the family is a crucial realm. Without the family, the state would deal with "inorganic" collectivities, masses that would easily succumb to despotism. Hegel is accompanied by the above-quoted Tocqueville, according to whom natural family ties are an antidote to the revolutionary fragmentation of society, and by Guizot, who saw the family as a model and prototype of democracy.

Such distinguishing and privileging of the family - the elementary family, let us add - nevertheless conceals a certain ambiguity. On the one hand, as Guizot claims, "family is a single entity whose elements are of secondary importance" (after Hunt \& Hall 1990, 103). On the other, however, these "secondary elements" are supposed to obtain full rights as individuals, also in relation to the family itself (to father or husband). This was the first step towards a vision of family not just as a "unit of reproduction," but as an association of free individuals joined by a web of voluntary ties and emotions: a miniature of the civic society. The family is supposed to be the first school of democracy, in which one learns the skills necessary to strike compromises and work together for the common good (although it seems difficult to define what this common good would be except for socialization, which prepares one to be a "good citizen").

All systems of social philosophy from that period presented the family already in its elementary form - a form that certainly was not the reality at the time, not even during the first stage of industrial society. As Louis Bergeron notes, "[1]arge private and, more recently, public industry is much less free of family domination than is generally believed. [...] Among business leaders family connections still determine career trajectories" (after Perrot \& Martin-Fugier 1990, 123). During Flaubert's trial in connection with the novel Madame Bovary, the author wrote to his brother Achilles: "They ought to know at the Ministry of the Interior that in Rouen we are what is called a family, that is, that we have deep roots in the region, and that in attacking me, especially for immorality, they will injure many people" (after Perrot \& Martin-Fugier 1990, 123; first emphasis preserved, second added). 
Since that time, transformations of the shape of the "family unit" would occur in two directions. On the one hand, it was still involved as an active actor in the dynamic changes of economic and social character - internal and external migrations, the development of new fields of economic activity, changes in the realm of work organization, and the severing of ties that make it dependent on "house" or "lineage." On the other hand, it became subject to intense influence: first, as an element of new social ideology ${ }^{241}$ and the object of active interference on the part of the nation state, and then, after the Second World War, as the object of research in the rising social sciences. Still, during the first stage of industrial society, the family unit - either indeed "shrunk to nuclear family" or discreetly extending its nepotistic ties in the field of economy - combined in itself all three dimensions of reproduction. The issue here is not only that companies have a family-like character but also that great industrialists-reformers would build a system of paternalistic ties, including construction of housing estates for workers, while professional education or transmission of skills and trades would occur inside families, not necessarily nuclear ones. What is more, despite the growth of the sector of universally accessible education, socialization would still take place within family, in accordance with models of family pedagogy, in which the father would play a dominant role.

Simultaneously, there unfolded the process that would later lead to the emergence of the last family configuration: the postmodern family, established through the very power of reflexivity. After all, just like the "social cell" of modernity, it is the creation of social ideology, to which the scientific diagnoses of experts are attached.

\subsection{Flourishing of industrial society and criticism of bourgeois family}

Whereas the nineteenth century was the period of "family triumphant," the second half of the twentieth century, especially the time after the Second World War, brought about devastating criticism of it. It seems that the idealized family of the nineteenth century did not perform well as a "civic mini-society" since it brought about the hecatomb of two wars, a youth rebellion, and the downfall of authorities. "Lawrence K. Frank, drawing on the studies of Margaret Mead,

241 The novelty rests in both the very phenomenon of ideology as a means of mobilizing and introducing coherence to the growing population, and the fact that it was the first time that "society" became an object for ideology. 
Ruth Benedict, Gregory Bateson and Erik Erikson, compared the 'affection, tenderness, and benevolent patience' shown to children in primitive cultures with child-rearing practices in the West, where the child was 'terrorized, humiliated, and often brutalized.' Such practices, he maintained, produced the 'aggressive, destructive, exploitive individuals from whom Western European culture has suffered for so many centuries'" (Lasch 1977, 103).

The critical analysis of the family, especially the American one, was done by representatives of basically all directions in social sciences: the Frankfurt School with the concept of "authoritarian personality" as a product of disciplining mechanisms operative in the family; psychoanalysis with the idea of "neurotic personality" developed in the coldness of the family house; as well as structuralists, functionalists, and even interactionists. The belief that the family is weak - spread in mass media and by the rising army of specialists and therapists - was incorporated into the self-consciousness of both parents and children, deepening the uncertainty as to the former's competences. Parents were treated in a particularly harsh way in the heyday of the romantic vision of youth. "The director of the Association for Family Living, Evelyn Millis Duvall, complained that 'only one profession remains untutored and untrained - the bearing and rearing of our children.' Parenthood, in her view, remained the 'last stand of the amateur'" (Lasch 1977, 108).

The radical shift of position, which led to the rejection of the ideal of "bourgeois family," was certainly an answer to the structural pressures heralding the twilight of industrial society. One could even say that - as in the case of the weaving of events and feedbacks, which created the bourgeois and proletarian family in the form of elementary family, although still enmeshed in a discreet web of nepotistic interrelation ${ }^{242}$ activated when necessary - we once again deal here with an entire set of interdependent factors. From the perspective of the present subject, there are four dimensions of these changes that seem crucial.

The first one is the increasing isolation of family from its surroundings, stemming from the collapse of neighborhood, lengthening of time spent outside home, as well as the increasing sense of alienation ${ }^{243}$ and hostility towards "society." The more intimate, personal and private, the family space becomes as

242 Blood relations played a particularly important role in migrations. It suffices to recall the phenomenon of Irish policemen in USA. Cf. also Finch (1989).

243 This sense was, to a large degree, developed not only by the media, but also by social sciences, which objectify the social reality and create a vision of society as a "steamroller" that crushes one's individuality. 
an alternative to and a shelter from the brutalized "outside" world, the more it becomes uprooted from the social order. As a result, nothing can become nested in it, nor does it itself become part of any greater whole. This also means that family's relations with its surroundings become increasingly "antagonistic" or even discontinuous ${ }^{244}$ - in line with the ideology of family as "a safe haven in this ruthless world."

The second is the expansion of the hired work market - basically the only possibility (with the exception of rentiers and landowners) to gain means necessary to survive. This caused the conceptual category of "labor" to change too. It began to be associated solely with gainful employment, making "household chores" not only worthless but also a field of conflict. Labor was extracted from home and the recommendation was to abstain from "bringing work back home," either in the sense of things that need to be done or a bad mood. Thus, labor became conflicted with home: days cannot be stretched indefinitely and every hour invested in work means one hour less for home. In this way, labor came to dominate over the family, especially in the case of women. ${ }^{245}$ "The most important indictment of the present organization of work is that it forces women to choose between their desire for economic self-sufficiency and the needs of their children. Instead of blaming family for this state of affairs, we should blame the relentless demands of the job market itself. Instead of asking how women can be liberated from the family, we should ask how work can be reorganized - humanized - so as to make it possible for women to compete economically with men without sacrificing their families or even the very hope of a family" (Lasch 1977, xvii). The demands of the job market also impose the necessity to make other choices regarding mobility, availability, "integration" with the work team, while labor itself goes through significant changes. ${ }^{246}$

The process of separating "workplace" from home was accompanied by the process of separating the family from the "place of education." In a way, the absence of parents at home was one of the factors behind the lengthening of the time spent by children in school, and the development of the sector of services "compensating" for the absent mother, which in turn could facilitate further

244 It is important to remember that we change identity whenever we leave home.

245 Work is obviously an indispensable source of means to support family. It needs to kept in mind, however, that the ever-expanding marketing makes more and more goods "indispensable," either for the child's proper development, or for securing one's social position. Although families were also subjected to economic pressures in the past as well, they were less subordinated to labor as a sphere separated from family life.

246 This shall be discussed in the next chapter. 
lengthening of her absence. This significantly changed the internal space of family life: in some way, "no one's home." The space of home is also emptied as a sphere of action: "there's nothing to do" at home except for "resting" (i.e. watching television). Even "recreation," "free time" and "hobby" have been moved outside home. In other words, "life goes on elsewhere." The banality of what is left at home - doing the washing, cleaning, and cooking ${ }^{247}$ - does not suffice to create a common "task" or goal that would seal a sense of unity. Thus, the family loses a sense of unity with regard to its shared goals, ceasing to hold specific functions except for meeting the emotional demands of household members.

Third, dynamically developing sciences enter the realm of the family. On the one hand, we have medicine and pharmaceutics, providing reliable contraceptives on a mass scale (for the first time in history). Their significance cannot be overestimated - not only do they provide control over reproduction, which can be subjected to rational planning, but also make such decisions an act of the individual will, one that does not necessitate negotiating with the partner. ${ }^{248} \mathrm{An}$ independent voice was also won by social sciences problematizing the family and formulating its normative ideal. In scientific criticism of family, a significant role began to be played by feminist movements as well as ones striving for the emancipation of minorities (particularly sexual ones). From the perspective of new, reflexive models, new ideals of family life have been formed: partnership, exchanging of roles, autonomy of individuals and the principle of dialogue ${ }^{249}$ instead of "disciplinary training."

This is particularly clear in the area of upbringing. One could say that all knowledge and competences necessary to complete this task have been appropriated by "professionals," which left parents with nothing but a sense of guilt. The famous handbook by Spock advises parents to listen to their hearts, but at the same time warns about the terrible consequences of losing self-control. Words uttered without thinking in a fit of rage could destroy the child's self-confidence, scolding could lead to long-lasting problems, while failure to provide the child with love and a sense of security could do irreparable damage. Conflict is pushed outside of the family - a "good family" ideally sees none of it. The same happens

247 These are unprofitable activities and thus not falling into the category of "labor," which makes them fruitless.

248 Coitus interruptus, practiced before, as well as earlier condoms, all demanded man's cooperation. The pill and then the spiral, however, made women entirely independent of the will and decision of their partners.

249 As Lasch observes, not without a certain note of spitefulness (1977), the principle of dialogue is nothing else than a mode of "appealing to enlightened egoism." 
with discipline since the family is meant to be a space of love and unconditional acceptance, not one of violence or compulsion. The family thereby ceased to exist as a specific entity having its own rules, and instead became transparent. In this ideology, no important processes of social or economic character come together in the family. It is rather a place of self-realization and emotional fulfillment.

Fourth, welfare state was developed, featuring mighty social policies oriented, among other things, towards helping and "repairing" faulty families. As Charles Murray demonstrates in his controversial study (2015), such programs mostly brought about the opposite result, chiefly because they failed to acknowledge the specificity of the family as a social whole, instead orienting themselves towards individuals.

"The history of modern society," Lasch writes in his excellent analysis of the mythologized view of family, "from one point of view, is the assertion of social control over activities once left to the individuals or their families. During the first stage of the industrial revolution, capitalists took production out of the household and collectivized it, under their own supervision, in the factory. Then they proceeded to appropriate the workers' skills and technical knowledge by means of 'scientific management', and to bring these skills together under managerial direction. Finally, they extended their control over the workers' private life as well, as doctors, psychiatrists, teachers, child guidance experts, officers of the juvenile courts, and other specialists began to supervise child-rearing, formerly the business of the family" (1977, xx-xxi). According to Lasch, the modern industrial society has "proletarized the family," in a way stripping it of control (or competence) over all vital processes comprising the general reproduction of social units. ${ }^{250}$

Were we to take this diagnosis seriously, it would be necessary to assume that the "family unit" analyzed here ceased to exist because the three abovementioned fundamental systems of social reproduction are "nested" not so much in the family anymore as, rather, in individuals. In a way, this would mean a return to the tribal reality - where the elementary family was dependent and unstable - or to the fleeting reality of plebeian families under feudalism - where family played no role in the reproduction of the social system except for providing "naked people." 251 This seems to be the direction in which Beck's analyzes

250 The paradox lies in the fact that, at the same time, the family is still considered as the "original environment of socialization," one that is basically responsible for the totality of the individual's success in life.

251 Naturally, there is a great difference here too - in tribal societies the dependent family was a "unit" of the clan, while plebeian families under feudalism were "nested" in the 
point. He rightly observed in Risk Society that "[t]hought through to its ultimate consequence, the market model of modernity implies a society without families and children. Everyone must be independent, free for the demands of the market in order to guarantee his/her economic existence. The market subject is ultimately the single individual, 'unhindered' by a relationship, marriage or family" (Beck 1992, 116; emphasis preserved).

The stable nuclear family, one assuming the subordination of individuals especially women and children - with regard to the institutional distribution of roles and obligations, became dysfunctional under pressures of mobility, individualism and flexibility. On the one hand, in the historical form it was given in early modernity, family is in real crisis, which manifests itself in the rising number of divorces, in postponing of marriage, in the gradual departure from formalization of relationships, with people choosing cohabitation instead, or finally - in the shift of power relations between women and men, which is generally regarded as the twilight of patriarchy. On the other hand, however, the model of nuclear family - legitimized in the modern era - has been subjected to shattering criticism. As a result, both in real and symbolic terms, the "triumphant family" characteristic for the modern society is now falling into oblivion. Some view this as a crisis or even downfall of the family; others, however, regard this process as the emergence of a new, more reflexive form of the family.

Is it not the case, however, that the "downfall of the family" is simply the downfall of a certain myth of the family, while the "emptiness" of the deserted home is a mythologized counterpart to the equally unreal, vibrant life of the "social cell"? Similarly, is it not true that the "postmodern family," i.e. one reduced to the expressive function, constitutes a new myth constructed on top of real structural pressures? Just like with the question about the crisis of society, there can be no certainty here whether we deal with the real dissolution of a certain institution, or rather with the inadequacy of the intellectual model still used to describe phenomena that are entirely new and cannot be grasped in this way.

\subsection{Postmodern family}

There is consent among theoreticians of postmodernity - like Bauman, Beck or Giddens, to name just a few - that the postmodern family is a reflexive relationship between subjects, a relation of open and continuous character, and not

community of the local manor farm or estate. Currently, the "naked people" produced by families do not belong to any such overarching community. 
a normative social institution of the old kind, one imposing roles and duties on individuals. If that is so, then family demands ongoing work of a reflexive and emotional kind, while its internal relations have become "pure" (to use a term developed by Anthony Giddens), i.e. autotelic and not instrumental. "Of key importance here is the emergence of the 'pure relationship' as prototypical of the new spheres of personal life. A pure relationship is one in which external criteria have become dissolved: the relationship exists solely for whatever rewards that relationship as such can deliver" (Giddens 2006, 6). In contrast to personal ties in traditional and modern societies, pure relations are not anchored in the outside conditions of social and economic life. This means that the family is constituted to an ever-greater degree through emotional transactions, in consequence of which outside points of reference are replaced with mutual closeness and intimacy. It is not "society" but individuals that create a family, simultaneously creating themselves: both the stability of the relationship and the confirmation of their identity depend on the quality of their relations.

Family members, especially the "founding" couple, need to continuously engage with work that demands not only reflexivity but also technical competence. This imposes on individuals the kinds of limitations of which they were not aware before: commitment is necessary for the relationship to endure; however, anyone who becomes fully committed is risking that - in case of break-up he or she will be painfully hurt. Dangers involved with engagement are even greater since the postmodern, fluid world does not guarantee permanence of any relationships. The paradox lies in the fact that - as Bauman aptly puts it relationships "need to be only loosely tied, so that they can be untied again, with little delay, when the settings change - as in liquid modernity they surely will, over and over again" (Bauman 2008, vii; emphasis added).

Therefore, individuals have to yet cannot form stable relationships. As a result, first, family as a stable and long-lasting relationship becomes brittle or even entirely impossible; and second, this situation condemns individuals to an endless lack of fulfillment. Volkmar Sigush, a therapist quoted by Bauman, observes that "[a]ll forms of intimate relationships currently in vogue bear the same mask of false happiness once worn by marital and later by free love... [A]s we took a closer look and pulled away the mask, we found unfulfilled yearnings, ragged nerves, disappointed love, hurts, fears, loneliness, hypocrisy, egotism, and repetition compulsion... Performances have replaced ecstasy, physics are in, metaphysics out..." (after Bauman 2008, 46).

The model of the postmodern family - or rather its normative idea - emerges from the conviction that it ceased to perform traditional functions, mainly economic and social ones, for the sake of the expressive function; moreover, 
many emphasize its instability. It cannot be entirely ruled out, however, that not much has changed in this respect: the "postmodern" family still constitutes a unit reproducing the social whole but operates more discreetly, while the capitals transferred to the younger generations - including cultural, social and economic capital - have a much less material character. After all, it is still inside the "family" - including patchwork or incomplete ones - that there emerges, to use a theoretical term developed by Gary Becker (1991), "human capital" (children), while the selection of partners meets the criteria of rationality (Giza-Poleszczuk 2005).

It is not true that today's family is particularly brittle, or that this has to do with fluid postmodernity. Phenomena like low marriage rates, cohabitation, single motherhood, or "patchwork" families not only occurred before but were also often the rule. To quote some examples: in sixteenth-century Sweden, cohabitation rates were higher than today (Wojda 2003); "patchwork" families were the rule in periods of high mortality rates and simultaneous economic demand to secure family's completeness (Kula 1983); until the nineteenth century, most marriages in England had a consensual character, not a formal one (Stone 1977); finally, in the past the break-up of the family after the death of one of the spouses would force more children to live in an incomplete family or under stepparents than today.

In other words, the phenomena considered today to be signs of crisis appear as such only against the background of a specific, historically-determined normative ideal. If in historically distant epochs the concept of "lineage" or "house" allowed to legitimize "patching" families through adoption, remarrying, relations between relatives or redistribution of children, today the idea of a good relationship provides meaning to practices enforced by structural pressures. Let us recall Ulrich Beck's thesis quoted before: “Thought through to its ultimate consequence, the market model of modernity implies a society without families and children." If Beck is right, the "postmodern family" - both in empirical and ideological dimensions - greatly contributes to the materialization of this assumption. This means that it is still a unit reproducing collective order, although in the postmodern era it is the market that has come to determine this order. If so, this sheds new light on the sense of crisis experienced in relation to the family.

\subsection{Crisis of the family as crisis of society}

So, where does the crisis-focused discourse originate? It considers the fragility of contemporary family as a threat to the very foundation of society's existence. ${ }^{252}$

252 This suggestion appears, for instance, in Greenspan's theory discussed in Chapter Two. 
Moreover, there is a clearly felt rise in anxiety about currently observed transformations of family, as experienced by common people, and expressed by columnists, scholars in social sciences as well as representatives of religious institutions. All of this undoubtedly constitutes a symptom of certain social fears, although they could be inadequately formulated. Thus, one needs to pose the question about the reason for the emergence of such fears. It is easy to point to demographic or economic consequences of the "family's crisis," e.g. negative birth rate and resultant ageing of the population as well as an unfavorable proportion of those working to those receiving pensions, or the related policy of accepting migrants from "foreign" cultures. Is it possible, however, to identify any specific dangers entailed by the weakening of family relations, and carrying implications for the functioning of society as such, or for its foundation that conditions the possibility of living together?

A question like this allows us to immediately notice that the main axis crystallizing the attitude toward the "postmodern family" is the question of whether one considers it from the perspective of social needs or individual needs.

In all previous ideologies (or representations) of the family, it was the first approach that prevailed - one giving primacy to the needs of communal living. In a society understood as a structure of collective actions, a strong and stable family was the source of order: a specific "social police" guaranteeing the minimization of "loose people," i.e. ones not incorporated into social structures and therefore possibly dangerous. "Prisons and boarding schools, barracks and convents, vagabonds and dandies, nuns and lesbians, bohemians and toughs, celibate individuals and institutions, often were obliged to define themselves in relation to family or on its margins. It was the center; they were the periphery" (Perrot \& Martin-Fugier 1990, 97); thus, "[t]heir physical and moral lives were complicated. Suspect or accused, they dwelt on the defensive, caught in a net which, though still relatively loose, steadily tightened" (259). It was the family's task to "bind" and somewhat pacify the centrifugal tendencies among individuals; ${ }^{253}$ to motivate them to adapt conformist patterns of behavior; and finally, to provide "energy" for even more intense labor. ${ }^{254}$

253 Those workers who were "burdened" with responsibility for the family's existence rarely displayed a revolutionary attitude; they were rather ones among whom strikebreakers would be recruited (MacKinnon 1991).

254 On the other hand, however, this paradox has been noted by authorities throughout history. The family is indeed simultaneously a "subversive organization" (Pinker 1998) that easily departs from rules of social life in the name of nepotism. 
In this perspective, family is not only social police but also social insurance. By creating a web of self-help, family aids the individual in finding solutions to difficult life situations without engaging public structures of help or social care ${ }^{255}$ (cf. Giza-Poleszczuk 2000). Even those who recognized the significance of the family for the development of the individual - like Freud, among others - placed emphasis mainly upon civilizing and restraining natural impulses that threaten social order. ${ }^{256}$ Although Freud's theory paints a picture of the family torn by conflicts and tormented by ambiguous feelings, its primary function was still to develop a superego and thus impose normative limitations on the individual.

In the context of this approach, the crisis of the family has entailed the rise of the number of "loose" and insufficiently socialized people, who are a potential economic burden, possibly lowering the degree of social integration, or even posing a threat to the public.

In contemporary considerations of the family, greater emphasis is placed on the role it plays for the individual rather than the society. It is primarily the individual who needs a "good family" that is supposed to not only constitute an irreplaceable source of security and stability, but also provide inspiration for development and self-realization. The family "socializes" only in the sense of laying the foundations of personality - not by "binding" and "civilizing;" it creates persons individualized, who exist only for themselves, not for any kind of "society" and not even for family itself, towards which they would have some obligations. The family understood as an institution based on cooperation, or even interdependence, does not fit this image at all.

"In liquid modernity," Bauman writes, "[...] it is no longer the task of both partners to 'make the relationship work' - to see it work through thick and thin, 'for richer or poorer', in sickness and in health, to help each other through good and bad patches, to trim if need be one's own preferences, to compromise and make sacrifices for the sake of a lasting union. [...] What follows is that the assumed temporariness of partnerships tends to turn into a self-fulfling prophecy. If the human bond, like all other consumer objects, is not something to be worked

255 Contradictory tendencies arise in this case as well: a self-sufficient family weakens centralized power, at the same time eliminating the field in which other groups could be formed, including associations, which constitute the basis of "civic society."

256 It is worth emphasizing here, however, that in Freud's theory the family does not constitute "a safe haven in this ruthless world" but rather a space of specific conflicts as well as ambiguous or negative emotions. Such a vision of the family fits well with those currents that criticize the family: Marxism, neo-Marxism, and some strands of feminism. 
out through protracted effort and occasional sacrifice, but something which one expects to bring satisfaction right away, instantaneously, at the moment of purchase - and something that one rejects if it does not satisfy, something to be kept and used only as long as (and no longer than) it continues to gratify - then there is not much point in 'throwing good money after bad', in trying hard and harder still, let alone in suffering discomfort and unease in order to save the partnership. Even a minor stumble may cause the partnership to fall and break down; trivial disagreements turn into bitter conflicts, slight frictions are taken for the signals of essential and irreparable incompatibility" (2006, 163-164). The ideology of individualism, which to a certain extent legitimizes the structural pressures necessitating "individuation," requires one to regard all forms of collectivism with suspicion because they limit individual autonomy. This includes the family as a supraindividual entity. As a result, whereas family was the specific "social police" in the past, guaranteeing the preservation of social order, today this role is played by public institutions that guard individual rights against threats arriving from inside the family.

Thus, it becomes clear that the heart of the matter is a question much more fundamental than the one of whether family is undergoing crisis or not. In the ideological dimension, the issue is about legitimizing individualism, not only in the ethical aspect, but also in the institutional one. Hardly anyone seems to doubt that structural pressures favor neither establishing a family nor securing its stability. However, the question is whether we shall consider these tendencies as a sign of the emergence of new forms of family - thus legitimizing them - or whether we shall treat them as a crisis-caused anomaly. Those in favor of the latter option indicate that it is absolutely necessary for the functioning of society to restrain egotistic impulses and reinforce the "moral sentiments" that incline us to be unselfish; this in turn demands subordinating individual needs to social demands. The best field for practicing this "social organ" - as Tönnies calls it is none other than family. Although this training does involve some suffering, misery accompanies individualism too. As Bauman observed with insight, "being an individual de jure means having no one to blame for one's own misery, seeking the causes of one's own defeats nowhere except in one's own indolence and sloth, and looking for no remedies other than trying harder and harder still. Living daily with the risk of self-reprobation and self-contempt is not an easy matter" (Bauman 2006, 38). Therefore, the question is not about the crisis of the family but about the thesis arguing the primacy of family (and by the same token, society) over the individual.

When expressed in the language of sociological theory, the debate on the crisis of the family is indeed an old dispute about the status of the concept of 
society. It is also easy to note that it goes hand in hand with the controversy indicated in Chapter One - over the question of what is it, in fact, that undergoes crisis: society or sociology, as well as over the question of whether it is possible to have a society comprised of individuals that are not rooted anywhere, or whether it is actually impossible to think about society differently than as an entity formed by smaller social sub-entities. If our assumption about society being a hierarchically nested system is true, it would be impossible to speak of any crisis of the family, regardless of how different its form is today. Although there can be no doubt that the "postmodern family" emerges in the context of structural pressures characteristic of contemporary times, it simultaneously allows them to expand through specific forms of socializing processes leading to reflexivity, fluidity and mobility. It is worth to recall here as well that family still constitutes to be the vehicle for economic, social and cultural reproduction, although the means it has at its disposal to achieve these goals are way subtler than marriage control, entail, or patriarchal power over women and children. 


\section{CHAPTER SIX. TRANSFORMATIONS OF LABOR}

What we are confronted with is the prospect of a society of laborers without labor, that is, without the only activity left to them.

HANNAH ARENDT, The Human Condition

Every concept of order divides and establishes the boundaries of what is acceptable; every concept of order leaves a part of society on the other side of the border - and openly voices the dilemma of its assimilation or parting ways with it for ever.

ZYGMUNT BAUMAN, Unnecessary and Rejected, or the Poor in a Rich World

The category of labor belongs to sociology's classic problems, occupying also a prominent place in theoretical systems developed within this discipline. It appears in the title of Durkheim's first book (2013), which attempts to derive the transformations of social solidarity from changes in the division of labor. In Weber's view (2005), shifts in attitudes towards labor are one of the main reasons behind the rise of capitalism - a socio-economic formation that is completely different from traditional society. In The Philosophy of Money, Simmel (2005) continues to return to labor as a factor whose fluctuations have affected the value of objects, people, and actions. All these thinkers convincingly demonstrate that the concept of labor involves a series of problems that are central to sociology (cf. Poleszczuk 1991), primarily because it combines the levels of individual actions and systemic properties, or - as in Simmel's theory - the domain of individual experiences (subjective, personal value) and their social standardization or objectification (value expressed in money).

A keen interest in the question of labor has been also taken by contemporary sociology, although it made the issue acquire a different character. Labor has become a self-contained object of study, treated as a separate area of human activity carried out in specific social organizations. The social dimension of labor is now analyzed primarily by studying relations among people employed in a single workplace, and their attitude to labor as such. Sociologists of labor focus 
on its forms, ways of organization, workers' relations, values connected to labor, as well as unemployment considered - almost by definition - as complementing the discussed concept.

Despite the significance attributed to labor, both as a theoretical category and as a distinguished area of activity, analyzed empirically, it remains difficult to define what it is. Is gathering of roots labor? Is hunting for buffalo labor? Can we say that labor is more like chipping stone pieces to give them the kind of shape that would make it easier to scrape meat from animal skins? Most people would find it more natural to provide a positive answer to the last question rather than the first two. In a popular view, labor is associated with creating tools and transforming the natural environment, while hunting, and especially gathering, seem to lack the above features ${ }^{257}$ - not to mention that also animals perform them. ${ }^{258}$

Still, attempts to approach the definition of labor in a more disciplined, scientific manner have brought more questions than answers. It is assumed today that "the essential feature of human labor is that its direct or indirect goal is to acquire means necessary to sustain the existence of both humanity as well as the world it has created and adopted, increasing chances for survival in a specific environment by controlling and transforming it" (Kozek 2002, 174). Clearly, such a broad definition of labor basically covers all forms of human activity that are not related to rest. This corresponds to the division into labor and leisure as two basic areas of human activity in contemporary societies. Yet, this dualism also accentuates the presentist character of such understanding of labor in sociological analyzes, i.e. one limited to a specific form of social order. Meanwhile, all data available to both scientists and amateurs unequivocally indicates that decisions about what is and what is not labor are strongly determined by historical and cultural factors.

It is precisely this aspect of human activity that Hannah Arendt emphasizes. "The world in which the vita activa spends itself," she writes, "consists of things produced by human activities; but the things that owe their existence exclusively

257 However, it ought to be said that both activities, even in their simplest form, necessitate the use of proto-tools (stones or sticks to kill animals; vessels for storing gathered fruit, seeds and roots). Moreover, both can greatly affect the natural environment, which is indicated by hypotheses formulated by researchers studying human prehistory.

258 It is the connection between labor and creation of tools that lay at the foundation of the well-known Marxist thesis that labor has created man. As Hannah Arendt notes, it was "one of the most persistent ideas of Marx since his youth"; however, as she points out, "Hume, and not Marx, was the first to insist that labor distinguishes man from animal” $(1998,86)$. 
to men nevertheless constantly condition their human makers. In addition to the conditions under which life is given to man on earth, and partly out of them, men constantly create their own, self-made conditions, which, their human origin and their variability notwithstanding, possess the same conditioning power as natural things" $(1998,9)$. The list of those "things" - "works and deeds and words" (19) - ought to include the socially created concept of labor as a factor that in turn conditions not only human actions but also forms of sociality.

Arendt uses the term vita activa to define three fundamental kinds of human activity: labor, work, and action. She considers them as basic since each corresponds to one of the three fundamental conditions of human life. Labor is an activity conditioned by the necessity to sustain the biological existence of human organism. Work refers to the world of culture, which is treated - as we do throughout this study - as an extension of, and simultaneously condition for the emergence and sustenance of a human-specific kind of mentality. ${ }^{259}$ Finally, action, according to Arendt's argument, is related to broadly understood politics. It would be conditioned by the fact that "men, not Man, live on the earth and inhabit the world" (xii); thus, decisions taken by individuals have consequences not only for them but also for other members of the collective.

From our perspective, what seems most interesting about Arendt's views is the analytical distinction between labor and work. Even if we treat all three kinds of the above activities as fundamental conditions of human existence, one could immediately assume that labor is in fact "more fundamental" than others for at least two reasons. First, biological sustenance of human organisms constitutes the necessary condition for becoming engaged in other forms of activity. Second, in the way that Arendt understands it, labor combines in itself the pre-social phase of humanity's history and the socialized "human condition."

For both reasons, such a narrowed concept of labor ${ }^{260}$ seems to be particularly suitable for analyzing the transformations of forms of sociality. Although labor

259 Arendt remarks that "[w]ork is the activity which corresponds to the unnaturalness of human existence, which is not imbedded in, and whose mortality is not compensated by, the species' ever-recurring life cycle. Work provides an 'artificial' world of things, distinctly different from all natural surroundings" (7). Both this explicitly articulated understanding and her later analysis of work as a specific form of activity allow to treat the term proposed by Arendt as one referring to actions connected with the sphere of culture (in the sense assumed in this study), which is related in this particular case primarily to the production of tools and knowledge.

260 It is important to emphasize again that we do not take this narrowed understanding as the definition of labor, especially one under the conditions of modern society, but 
has accompanied people since always (just like family, analyzed in the previous chapter), it can be variously understood and organized, which entails that it can in turn variously condition the form of social bonds and the specifics of accompanying obligations.

Therefore, let us examine the transformations of human labor understood in this way.

\subsection{Labor as an element of being in the world - hunter-gatherer societies}

In a provocatively titled essay “The Original Affluent Society," Marshall D. Sahlins argues that hunter-gatherer societies serve a double role in social sciences. First, they constitute a negative example of so-called survival economy. Second, they offer the key argument in treating the history of human societies as one constantly progressing in terms of development, not only economic but also civilizational. He bitingly remarks: "Almost universally committed to the proposition that life was hard in the Palaeolithic, our textbooks compete to convey a sense of impending doom, leaving one to wonder not only how hunters managed to live but whether, after all, this was living?" $(1972,1)$. Therefore, for both these reasons, it seems that the main function of these societies is to make contemporary researchers feel better (and their potential readers too), allowing them to look around and say (some actually do so): "Well, look where we are now thanks to human ingenuity!"

This specific variant of "negative PR" against primal societies studied by Western European travelers and scientists had its roots in the use of European criteria to describe other cultures, which hardly happens these days. However, improved methodological standards fail to protect scholars from another pitfall - one related to the fact that contemporary tribal communities studied by contemporary scientists substantially differ from their Palaeolithic prototypes. Sahlins points out this difference clearly when he writes that "the anthropology of hunters is largely an anachronistic study of ex-savages, an inquest into the corpse of one society, Grey once said, presided over by members of another" (1972, 8; emphasis added).

rather as a tool that makes it possible to achieve greater precision in demarcating this area or aspect of human activity, which is the subject of analyzes in this chapter. It seems obvious to us - just like it seems to have been to Arendt - that labor understood in this way cannot be in fact separated from either "work" or "action" in the sense she gave to these terms. 
Notwithstanding the above, let us attempt to say something about the place occupied by labor in the earliest form of human communities by referring to anthropological studies. It is universally assumed - hence the name of this kind of social organization - that these communities relied on two kinds of activities: gathering, done mainly by women, and hunting, which was the domain of men. However, as Jared Diamond points out, the share of hunting in the provision of food is overestimated with regard to these communities (especially in relation to proto-human collectivities from before the Great Leap Forward), at least because hunters would usually catch smaller animals - ones that would only round out a diet whose main component were plants (1991, 33-35). Analyzes presented by Sahlins lead to similar conclusions. Based on data acquired in the middle of the twentieth century, his research shows that in longer stretches of time it was the gathering done by women that provided the whole community with the major portion of food.

As Sahlins demonstrates, finding and preparing food would take members of these societies ca $4-5$ hours a day; thus, "they do not work continuously. The subsistence quest was highly intermittent. It would stop for the time being when the people had procured enough for the time being, which left them plenty of time to spare" (17). It is worth emphasizing here that this type of labor was not limited to securing simple survival because a portion of time allocated to it would be taken by searching not just for any food but for its particular types. The rest of time would be devoted to sleep, rest and "social" activities. ${ }^{261}$ And even though finding food was the basic occupation in hunter-gatherer societies, in overall balance it took relatively little time and had a discontinuous character in the sense that it was intermingled with frequent periods of sleep and rest.

From the perspective of the role played by this mode of sustaining biological existence in creating a specific form of sociality, which is found - regardless of later modifications - in contemporary tribal communities, it seems to be of greatest importance that labor does not create in them a separate subsystem, one that would be disconnected from other forms of activity. Despite the existence of a sex-based division of tasks, there is no specialization in hunter-gatherer communities, and no division between labor and work. Necessary tools are produced by everyone who needs them at a given moment. Even if there do emerge individuals distinguished by their agility or some specific skill (sculpting, making nets or arrowheads), their talents do not relieve them from participating

261 In languages used by certain Australian tribes, "work" and "play" are designated with the same word. 
in actions that form the basis of labor in the entire community. The same is true for the tribe's chieftain, who is appointed due to individual characteristics but participates in communal life on the same terms as everyone else.

Furthermore, despite the fact that hunting and gathering - part of common knowledge transmitted from one generation to another - are obviously socialized, they are also subordinated to physiological rhythms rather than economic ones. Phases of labor are interwoven with phases of rest in accordance with the daily rhythms of hunger and satiation, without even considering the future, i.e. without gathering surplus food for future needs in case of a shortage. In other words, labor does not create separate forms of sociality, ones that would belong only to this form of activity. It does not create social divisions or social regulations proper only to it. In that sense, labor is woven into the everyday rhythm of being in the world on the same terms as it occurs among other species.

It is possible to put it in even stronger terms: if the search is after the factor responsible for the "creation of humanity" during the Neolithic Revolution, then contrary to the belief held by Marx, it would not be labor - hunter-gatherer communities that survived until today are by all means "human" or, to use our preferred terminology, "socialized" in all aspects of their existence, despite the fact that labor is organized in them in a way conditioned by features of the habitat, and subordinated to regulation of biological needs. Demands of labor do not possess their own driving force in these collectivities - one that would be capable of modifying other aspects of communal life in a way that would distort the socially generated state of balance. They are woven into the order of nature rather than into the economic one.

This state of balance - in which a handful of hunter-gatherer tribes still last, living in relative separation from outside influences - was shaken only by the transition to agriculture and the adoption of a settled lifestyle. Certainly, this was not a one-time event, but a process initiated independently in several places on earth. It unfolded slowly: since the emergence of agriculture in the Middle East ca 8,500 BC, two thousand years had to pass until it reached Greece, and further two and a half before it found imitators in Britain and Scandinavia. Moreover, contrary to the popular view linking this process with "progress" induced by the development of human mind, data available today show that the shift actually worsened the "quality of life" among food producers rather than improved it in comparison to hunter-gatherers.

Both Sahlins and Diamond underline that even if we take into account contemporary standards related to the sustenance of biological existence, the hunter-gatherer form of labor had many advantages over any later ones. Sahlins quotes estimates of energy balance calculated on the basis of daily food rations, 
which show that these amounts more than meet today's criteria, also with regard to specific nutrients. Further, he draws attention to the incomparably greater amount of free time enjoyed by individuals. Diamond, in turn, referring to the results of research conducted by paleopathologists, indicates that the shift from the hunter-gatherer to the agricultural mode of obtaining food had several negative consequences: decrease in the average height among the population; ${ }^{262}$ increase of the number of cavities and teeth diseases; increase in contagious and bone structure diseases; and finally, rise in mortality rates. ${ }^{263}$ Furthermore, the risk of hunger went up as well due to the dependence on one or several kinds of crops $(1991,170-172) .^{264}$

In light of the above data, the true mystery is not how certain forms of hunter-gatherer societies managed to survive until this day, but rather how it even happened that a fundamental change in the mode of obtaining food was made along with the popularization of agriculture. Hypotheses attempting to explain this process point to the coincidence of factors that are primarily related to transformations of habitat. These included, on the one hand, decrease in the amount of food obtained directly from the natural environment, which was caused by climate change and the extinction of certain species of animals, as well as the perfection of hunting skills among the primitive tribes and the increase of their size, which caused the habitat's resources to be exploited more intensively, in turn decimating animals and ultimately necessitating animal husbandry. On the other hand, the aforementioned climate changes led, towards the end of the Pleistocene, to the increase in the range of wild species of cereal crops in the Middle East, which, in turn, favored adopting these crops more frequently as a dietary basis (Diamond 1999, 114-119; cf. also Saggs 1962, 3-10). Thus, it was

262 The average height of hunter-gatherers in the area of today's Greece and Turkey was, towards the end of the Ice Age, $180 \mathrm{~cm}$ for men and $168 \mathrm{~cm}$ for women, whereas ca $4,000 \mathrm{BC}$, when agriculture already dominated in those regions, these numbers amounted to $160 \mathrm{~cm}$ and $155 \mathrm{~cm}$, respectively.

263 Introduction of intensive maize crops ca 1,000 CE in America caused the per cent of people aged 50 or more to drop to 1 per cent in comparison to 5 per cent before the popularization of these crops.

264 In hunter-gatherer societies (naturally, those that live in a relatively natural habitat) there are better or worse periods in terms of food amounts, but there are no instances of devastating hunger since a varied diet allows to replace some forms of food with others. The best-known example of tragic consequences of dependence on a monoculture of crops is the Great Famine in Ireland (1845-1847), during which potato blight caused around a million people to die and forced a half of the remaining population (ca 1.5 million) to emigrate. Cf. more on this subject in Hobhouse 2005. 
necessity, on the one hand, and opportunity, on the other, that helped set in motion the process that came to be called the Neolithic Revolution. It initiated a long chain of events transforming labor into one of the crucial factors determining the form and content of socializing processes.

\subsection{Labor as a task - traditional societies}

In sociology, the term "traditional societies" is used in reference to all those forms of social organization that existed before the emergence of modern society. This is justified both by the discipline's tradition ${ }^{265}$ and by certain features shared by actions and institutions encountered in premodern societies. They include the dominant role of religion in determining collective representations, the centrality of agriculture to economy, the rule of a king or a chieftain in the political sphere, a low pace of changes and the subordination of individual actions to the patterns of behavior passed from one generation to another. Without negating the existence of certain similarities, it is worth to remark here that using a single semantic category to refer to societies as different as primitive hunter-gatherers (who did not know agriculture), ancient empires, Greece and the Roman Empire, and, finally, feudal Europe, makes it more difficult to discern the complexity of processes hiding behind the transformations of dominant forms of sociality. ${ }^{266}$ This becomes particularly inconvenient when considering processes linked to transformations of the economic order and the resulting changes to both social structure and attitudes toward labor as such.

In the first period of the transition to agriculture and settled life, nothing really heralded the immense scale of transformations induced by the change in the mode of obtaining food. As we have stated above, it was forced, unfolded slowly and entailed a clear decrease in quality of life since the profits gained from producing food were quickly equalized or literally consumed by the rapidly growing population. The settled lifestyle enabled to shorten the period between subsequent births, which led to a growth of population that would often exceed the rise in food production. Nevertheless, it was exactly the relationship between food production and population growth in a given society that turned out to be the decisive factor in the acceleration of changes. After all, "higher

265 We mean here primarily works by Durkheim, Tönnies, and partially Weber; they all focus on the moment of the transition to modernity, which favors assuming a bipolar division.

266 However, it makes it easier to treat human history as a steady line of progress leading from primitive communities to Greeks, Romans, and the contemporary world. 
birthrate of food producers, together with their ability to feed more people per acre, lets them achieve much higher population densities than hunter-gatherers" (Diamond 1999, 89). Thus, the latter began to be driven out from their territories or were forced to give up their nomadic lifestyle. ${ }^{267}$

As discussed in Chapter Four, one direct outcome of population growth was the change in the organization of social life, which involved replacing tribal systems with chiefdoms. In the Middle East, the latter emerged already ca 5,500 BC, giving rise to a division that still accompanies humanity: the division into those who work and those who do not, the latter obtaining means for the sustenance of their biological existence from other sources or forms of activity. At many stages of human history, this division structured societies in a variety of ways, but has invariably constituted an important factor in shaping social bonds, and consequently - the forms of socializing human actions. For this reason alone, one ought to consider separately the cases of ancient empires, Greece, and European feudalism. After all, they represent diverse ways of solving problems related to the transition from chiefdoms, based largely on tribal communities, to more complex systems of social organization. Such a distinction will help identify the influence that transformations of labor had on the development of this general form of sociality we have come to call the traditional society.

Historically speaking, the earliest ancient empires rose in Mesopotamia, which is understandable given the fact that the region between Euphrates and Tigris was one of the first where the Neolithic Revolution started. ${ }^{268}$ The oldest remains of settlements found in this area date back to ca 5,000 BC. From the perspective of labor, it is interesting that they differed from hunter-gatherer communities primarily in having developed early forms of specialization in everyday activities of their inhabitants. However, these settlements were relatively small, comprising just several larger families. Isolated and economically self-sufficient, they would be led by an elected chieftain. They established contacts with others only gradually, by initiating trade. A truly revolutionary change was started only by the rise of city-states.

267 "Those few peoples who remained hunter-gatherers into the twentieth century escaped replacement by food producers because they were confined to areas not fit for food production, especially deserts and Arctic regions" (Diamond 1999, 113).

268 The area is called the Fertile Crescent. Diamond (1999) lists four additional regions: China (Yellow and Yangtze Valleys of China), Mesoamerica (today's central and southern Mexico, and adjacent parts of Central America), the Andes, and the eastern part of today's USA. In each of them, the transition began later than in Mesopotamia. 
In his discussion of the earliest period in the history of Mesopotamia, Henry W. F. Saggs argues that " $[t]$ he aggregation of communities in the south into cities was almost certainly dictated by the rivers: to control and utilize them effectively needed cooperation on a larger scale than small isolated primitive villages could provide" $(1962,19){ }^{269}$ Credit for the organization of the first city-state in this region is given to the Sumerians. It was in their cities that - at the threshold of the third millennium BC - there developed an economy based on full-blown specialization. As Saggs points out, "[t]wo aspects of this specialization with almost immeasurable consequences for the subsequent activities of Man were the development on the one hand of the arts and on the other of a social group professionally concerned with the religious response of Man to the pattern imposed on his life by the agricultural cycle" (7).

Sumerian city-states initially had a theocratic character, with land belonging to the temple, and the lay chieftain of the city being a representative of the local deity, managing the temple's property with the help of an army of subordinate officials. ${ }^{270}$ This property would include not only land but also tools and animals necessary to cultivate it, as well as craftsmen's workshops, mills and breweries. Land was partially leased, but large portions of it were worked by hired people, paid either in precious metals or food products. The entire economic activity would be centralized, with management based on a bureaucratic system: specialized teams did the work directed by appropriate offices, while strict bookkeeping stimulated the development of writing.

With the passage of time, secular authorities would gain greater autonomy. Along with the consolidation of power by rulers of city-states, most agricultural areas would become property of the king or - strictly speaking - of the state. ${ }^{271}$ However, this did not change much in the division of labor, except for the rise of an elaborate state administration. Basically, everyone would work, even the

269 Anyway, all "agrarian bureaucracies" were dependent on rivers carrying fertile silt (Egypt), on canals irrigating desert-like areas (Mesopotamia and Persia), and on maintaining elaborate irrigation systems (China).

270 This office was initially filled in the course of an election from among the city's free citizens, and became hereditary only since the middle of the third millennium BC.

271 This process was related to a very complex, three-thousand-year-long history of the region, during which centers of power moved from the cradle of the Sumerian state the city of Uruk - to Ur, and were subdued at the turn of the second millennium by Babylon. In turn, since the eighth century $\mathrm{BC}$ Babylon was conquered by the Assyrians, but after the latter's fall in the seventh century BC it regained its former position for a brief period before surrendering to the invasion of Persians led by Cyrus. 
ruler's party would have its own duties between military campaigns, engaging in works related to maintenance of canals and construction. Craftsmen were "temple's men" or "king's men," which meant permanent employment in one of the big workshops run by the two main centers of economic life (Bielicki 1966). They would be organized into guilds, in which "the elderly ensured a just distribution of commissions, controlled the quality of products, and were held responsible by the temple or the ruler for the raw materials received from magazines as well as for prompt supply of appropriate quantities of ready products" (289). Their position depended on qualifications and skills. In this strongly structured society, anyone who could not cope with assigned tasks would fall to the lower rungs of the social ladder.

The account of the functioning of the entire system, which emerges from hundreds of clay tablets containing receipts, lists and directives, resembles a description of a well-managed company whose profits are of course unevenly divided but whose large portion would be allocated to serve the public good, i.e. to construct a network of canals, roads, and further temples. Naturally, one could infer that - just like it happens in today's organizations of this type - the closer one gets to the top of the social ladder, the smaller role is played by the principle of meritocracy in favor of descent as well as informal relations and connections. This can be easily discerned at least from the fact that some rulers introduced rotation with regard to certain high posts (an equivalent to province governor would be periodically transferred from one region to another), thereby attempting to counteract the creation and congealing of local systems of connections. It should also be expected that the functioning of the entire bureaucracy would be strongly limited during frequent periods of invasions and struggles for power. Historians agree that along with subsequent waves of invaders, "the old Sumerian system of state-socialism had begun to break down” (Saggs 1962, 198).

In economic terms, this breakdown manifested mainly in the increasing diversification of wealth. Whereas in earlier periods temples were not only companies employing ranks of people but also supported citizens afflicted by hunger, natural disasters and disease by distributing food, with the course of time independent farmers, traders or craftsmen could only take out a loan at a high interest. "Over the years this would result in the greater part of the peasantry becoming the victims to a crippling load of debt, and the situation could only be cleared by drastic measures, namely by a general remission and a fresh start"272 (198). This

272 Descriptions of similar situations can be found in the Old Testament. Cf. Chouraqui 1971. 
would be done by the ruler enacting laws that would specify, among other things, prices of foodstuffs, farm laborers' wages, and charges for hiring carts or boats. As Saggs emphasizes, the basic goal of ancient legal systems was "to set forth justice in the land" (198), mainly in the economic sense.

If the social structure of Mesopotamia's ancient empires sometimes resembles elaborate classifications of professions used by contemporary sociology, while the various laws included in diverse "codices" bound men and women in equal $^{273}$ measure, then "the Greek city in its classical form was marked by a double exclusion: the exclusion of women, which made it a 'men's club'; and the exclusion of slaves, which made it a 'citizens' club'" (Vidal-Naquet 1986, 206). Slaves were also found in the social structure of Mesopotamia, but they played a chiefly economic role, i.e. as a source of cheap workforce; moreover, a free citizen could become a slave as well after becoming an insolvent debtor (Bielicki 1966). It was different in Greece. As Arendt shows, keeping slaves was not related to economic factors but to the desire to set oneself free from "the slavish nature of all occupations that served the needs for the maintenance of life. [...] To labor meant to be enslaved by necessity, and this enslavement was inherent in the conditions of human life" (1998, 83-84).

Thus, the status of slaves was primarily a derivative of the Greeks' attitude to labor, which they saw as a condition that humans share with animals. After all, the latter also need to struggle to obtain means for sustaining biological life, which makes it inhuman, i.e. not specific to the human condition. "The 'good life', as Aristotle called the life of the citizen [...] was 'good' to the extent that by having mastered the necessities of sheer life, by being freed from labor and work, and by overcoming the innate urge of all living creatures for their own survival, it was no longer bound to the biological life process" (Arendt 1998, 36-37). As Pierre Vidal-Naquet aptly notes, "[t]he slave made the [Greek] social game feasible, not because he performed all the manual labor (that was never true) but because his condition as the anti-citizen, the utter foreigner, allowed citizen status to define itself" $(1986,164)$.

The attitude towards labor developed by Greeks certainly evolved. Homer's heroes perform various tasks that could be evidently called labor: Paris and Odysseus help with building their houses, while Nausicaa herself washes the underwear of her brothers, which does not require any further explanation. ${ }^{274}$

273 Still, the latter would suffer worse consequences than men in the usual punishment for the same misdeeds.

274 Arendt explains this by referring to "the self-sufficiency of the Homeric hero, to his independence and the autonomic supremacy of his person." "No work," she concludes, 
During the archaic period - the setting of Homer's epic poems - there naturally were slaves in the Greek society. However, they did not occupy the lowest rung of the social ladder - this place was reserved for farm laborers, who had only a pair of hands at their disposal, and had no permanent ties to the farm (Vidal-Naquet $1986,160)$. "It is only from the late fifth century onward that the polls began to classify occupations according to the amount of effort required, so that Aristotle called those occupations the meanest 'in which the body is most deteriorated.' Although he refused to admit banausoi [craftsmen] to citizenship, he would have accepted shepherds and painters (but neither peasants nor sculptors)" (Arendt 1998, 8-182). However, it was already Hesiod (active at the turn of the eighth and the seventh centuries BC) who wrote that labor, just like any other evil, was released from Pandora's box as a punishment inflicted by Zeus on Prometheus for the fraud the latter committed against gods. Hesiod also found it natural and unworthy of justification that farm labor constitutes a preoccupation strictly for slaves and farm animals. The contempt for labor displayed by the Greeks, which emerged from the desire to liberate oneself from life's necessities, was only reinforced as a result of increasing duties related to participation in the life of the polis, and the pressure to abstain from any activities beyond those of public character.

At first glance, Romans seem to have partaken in the Greeks' attitude towards labor. The claim made by Cicero - "wage labor is sordid and unworthy of a free man, for wages are the price of labor and not of some art; craft labor is sordid, as is the business of retailing" (after Veyne 1992, 121) - does not differ at all from Aristotle's quoted above. However, Cicero also adds the following caveat to the last item: "as opposed to large-scale wholesale trade" (121). For Romans, being free from labor was not the equivalent of freedom, or at least not the only one; it was first of all an indicator of high social status. Wealth and descent counted most. That is why wholesale trade was not as "ignoble" as running a small shop, while a landowner who took up trade would not be treated like a merchant, although a tradesman who bought land would be long considered a parvenu.

This difference was reflected in dominant social divisions. In Greece, the basic structure involved division into citizens, metics (foreigners earning their living

"is sordid if it means greater independence; the selfsame activity might well be a sign of slavishness if not personal independence but sheer survival is at stake" $(1998,83$; note 7). However, this interpretation seems anachronistic since it does not take into account differences in ways of thinking, which distinguish the archaic and classical periods of Greek culture. 
as craftsmen or tradesmen) and slaves. In Rome, free citizens would be divided into "estates" - ordinary citizens, decurions, equites and senators. This division was based on wealth, whose main component was land. However, it was not material wealth in itself that decided about affluence, but rather descent. Slaves could hold high offices and make fortunes, becoming trusted confidants of their masters, managers of their estates, and teachers of their children, but were nevertheless prohibited from participating in public life. An impoverished descendant of a noble family could give lessons at the house of a notable and be treated as a friend of the house despite being paid for his services. As Veyne writes, "[i]n this society no one was a worker; all social relations were conceived in terms of friendship or authority" $(1992,125)$.

Nonetheless, the primacy of birth in the determination of social position does not mean that the Roman upper class was a "leisure" one. It is even possible to say that the lives of these people were filled with business, speculation, management of workshops or companies, lending money at usurious rates etc. In the writings of Roman thinkers, we also find much praise for labor and entrepreneurship. However, as Veyne underlines, Romans considered diligence to be a moral virtue, not really a factor lying at the root of social divisions. The differentiation between those who work and those who do not appeared primarily at the level of collective representations, not at the level of real differences between kinds of activities. "Only common folk worked for a living. People of quality managed, that is, they engaged in the activity referred to as cura or epimeleia, which one might translate as 'government' [...]. This was the only activity worth of a free man, for it involved the exercise of authority" (141). Even if such "government" took up all time available to a given person, and even if it entailed receiving remuneration or involved activities demanding physical effort, it was not labor as long as that person was of noble descent.

One could therefore say that, by virtue of this semantic trick, the Romans succeeded in combining in their attitude to labor two features that would seem entirely contradictory: the kind of deprecation typical of the Greeks, and the belief that "no labor is disgraceful as long as it brings large profits," an idea typical of contemporary capitalist society. According to Veyne, Romans went even further in their approval of labor than it happens today, in line with the principle "Any toil, no matter how pleasurable, merited payment" (147). ${ }^{275}$ Engaging

275 "One picturesque aspect of amorous customs among the Romans was that the female partner in a high-society affair was paid for her trouble. A matron who deceived her husband received a large sum or, in some cases, an annual income from her lover. [...] The practice of accepting gifts from lovers was considered not prostitution but 
one's own and other people's resourcefulness, pursuing profits, seeking favors of the high and mighty, and taking any opportunity that presents itself to multiply wealth - these tendencies blurred any differences between estates, uniting all in the effort to amass riches. If the group that had the best opportunities to achieve this goal was the nobility, it was because they had easier access to information as well as because the entire economic system was based on agricultural production, making land ownership the best and most reliable capital resource.

All of this changed radically when the Roman Empire fell and the Middle Ages began. Despite being based on an agrarian economy, the Roman world was essentially urban, while the mediaeval one was first and foremost that of the countryside (Le Goff 1970, 13). It was also dominated by a vision of reality forged under the pervasive influence of Christianity, which is based on entirely different principles than those informing Greek and Roman views. ${ }^{276}$ Finally, it was a world deeply marked by its tribal, "barbarian" heritage, which only to a certain extent yielded to the influence of "the mission to civilize" taken on by the Christian clergy, which considered itself the heir to Roman culture (Le Goff 1992; Modzelewski 2004).

At the same time, the very concept of the Middle Ages is internally "broken," both in its spatial and temporal dimensions. Different factors shaped forms of life on territories that had been part of the Roman Empire, and those extending east of the Rhine and north of the Alps and the Danube. Moreover, different factors played the leading role in the period up to the tenth century, on the one hand, and the period up to the fifteenth, on the other, which saw the budding of processes that transformed mediaeval feudalism into germinating capitalism. Keeping this in mind, let us nevertheless identify the most characteristic features that determined the place of labor in the feudal society.

The face of the medieval society was shaped not only by the dominance of agrarian economy - in fact, cultivation of land serves as the basis of economy in all kinds of traditional societies discussed here - but also by the ruralization of the population (Le Goff 1992). The destruction of cities during subsequent waves of "barbarian" invasions certainly contributed to this, but - as Le Goff emphasizes this was just the final note in a long process that began during the final stages of

work for hire. The woman did not give herself because she was paid, the jurists held; she was rewarded for giving herself of her own free will. She who loved best was most handsomely paid" (Veyne 1992, 147).

276 Of course, Christianity drew many themes from Greek thought, but gave them a different meaning. 
the Roman Empire. It led to the gradual limitation and finally disappearance of those forms of economic activity that keep cities alive: craftsmanship, trade, as well as material, intellectual and cultural consumption. This process had at its roots a complex weave of factors which conditioned the functioning of Roman economy. Among them an important role was played by the growing fiscalization of the state. Importantly, it led to the disorganization of exchange. "The disorganization of the exchanges increased hunger and the hunger pushed the masses into the countryside and subjected them to the servitude of the 'breadgivers', the great lords" (Le Goff 1992, 24-25).

Invaders became part of this economic structure effortlessly. First, they were no longer nomads, but "fugitive sedentaries" (27). ${ }^{277}$ Second, barbarian tribes had long abandoned the egalitarianism of their original communities. Despite its members still retaining the status of free men, barbarian communities were deeply differentiated in social terms, including "powerful and weak, rich and poor who easily transformed themselves into great and small proprietors or occupants on the conquered land" (28). And finally, third, the desire to retain their difference, together with the fear of melting into local population due to small size, ${ }^{278}$ inclined barbarians - at least initially - to avoid cities and to settle in villages, where it was easier to follow the old traditions, whose nature is best reflected by the so-called Salic Law. ${ }^{279}$

However, becoming part of the economic structure of the late Roman Empire did not mean that the state's ideas about the world, or its models of behavior were to be continued. As Le Goff notes, barbarians "turned a decline into a regression. They combined a threefold barbarism, their own, that of the decrepit Roman world and that of the old primitive forces which lay below the Roman varnish and had been freed by the dissolving of the varnish under the impact of the invasions. The regression was chiefly a quantitative one. The barbarians destroyed human lives, great buildings, and equipment necessary for the economy. The population fell sharply; art treasures were lost; the roads, workshops, warehouses, irrigation systems, and cultivated areas fell into decay. [...] In this impoverished, underfed,

277 Le Goff writes that "the medieval society was half-nomadic. Hunger for land, changing fates of conflicts, religious unrest - all inclined these people to mobility" $(1970,15)$. It was, therefore, a mobility enforced by specific circumstances of individual existence, not a lifestyle component.

278 According to estimates made by historians, the population of all barbarian tribes after settling on the territories of the former Roman Empire amounted to only 5 per cent of the total population (Le Goff 1992, 29).

279 Cf. more on this subject in Modzelewski 2004. 
weakened world a natural calamity succeeded in completing what the barbarians had begun" (31-32). It was the bubonic plague that - since $543 \mathrm{CE}$ - devastated for half a century Italy, Spain and large parts of the Gaul.

The bubonic plague - or, strictly speaking, its consequences - interfered for a long time with the weak process of acculturation, which began with the settlement of barbarian tribes on the territories of the former Empire. One could even say that these circumstances halted all forms of socializing processes. Thus, it was not only a period of return to paganism but primarily to sophisticated torture, excess and exceptional "despotism curbed by murder," a period when "direct regulation" would become dominant (Elias 2000), i.e. one based mainly on physical force or the law of the stronger. ${ }^{280}$

This period concluded in the eighth century when political power was consolidated, thereby allowing for the formation of a properly feudal society (cf. Le Goff 1992; Elias 2000). The process was extended in time: its economic and social effects began to be felt only at the beginning of the eleventh century. These effects included the crystallization of social divisions. During the early Middle Ages, the categories used to describe the society had a bipolar character. Depending on the context, they involved the following oppositions: clergy and laity, mighty and weak, free and slaves. In the eleventh century, there emerges another classification, "which was to become classic: those who pray, those who fight, those who labor: oratores, bellatores, laboratores" (Le Goff 1992, 256). Nevertheless, the concept of laboratores - as Le Goff shows - had a very specific sense: it "designated the upper level of the peasantry, the ones who owned at least a yoke of oxen and their work-implements" (259). Thus, it would not cover the masses of poorest physical workers, whose only tools were their own hands. A later reflection of this division is the one into the clergy, nobility and third estate, i.e. the bourgeoisie. This system also leaves no place for simple folk, who exist, as it were, outside society.

The emergence of the bourgeoisie within this tripartite schema is related to the transformations that occurred between the eleventh and the thirteenth centuries with the development of cities. During the early Middle Ages, there were basically few cities in today's sense of the word. Most of them, as we have already mentioned, were razed, ruined or deserted. The few that survived from

280 Le Goff provides a summary of this period: "people of the Middle Ages - here one can risk saying: all of them - desired one thing only: to escape from their epoch into the spirit world, to Heaven. Among all the different things that made them tremble, the fear of death was the weakest" $(1970,16)$. 
the Roman times lost their political and economic functions, owing their existence and relative prominence chiefly to having become the bishop's seat. New burghs were simply extended seats of notables, surrounded with walls that also protected people living in settlements outside them, which later grew, forming cities. The economy had a largely natural character, with the exchange of goods and products occurring mainly within local communities.

In this world of farmers and warriors, with clergy reminding the faithful about the futility of all earthly things, labor was considered as an activity of an unambiguously low status. It was despised both by knights and clergy, although the latter usually expressed this in a more veiled manner. "[I]n man's obligation to labor the church saw [...] the result of his imperfection [...]. The Fall entailed divine retribution" (Gurevich 1985, 259-260). ${ }^{281}$ According to Christian theologians, people ought to care more for the salvation of their souls than for survival and material prosperity. Thus, a contemplative life that brings man closer to God would be the most appreciated of all forms of activity. As Max Weber observed, it was already Thomas Aquinas who argued, while interpreting the claim made by St. Paul ("He who does not work, neither shall he eat"), that labor is necessary only due to the need to keep oneself alive. "Where this end is achieved, the precept ceases to have any meaning. Moreover, it holds only for the race, not for every individual" (Weber 2005, 105).

However, even in the early Middle Ages, the attitude towards labor was not as clear-cut as it might seem at first glance. As is often emphasized, labor plays a significant role because of its disciplinary character as well as the interest of the whole. Laboratores are an inseparable part of an organic vision of society, which could not exist without them. As Le Goff points out, "in medieval iconography clergy and aristocracy allowed peasants their own place not because of the size of that social group but because the reigning ideology desired to portray in art an image of society that would be not only hierarchical but also coherent and harmonious - such an image would naturally counteract any claims or revolts" $(1970,15)$. At the same time, emphasizing the significance of labor also had an educational aspect. "Idleness - the 'enemy of the soul' - breeds vice and threatens

281 As Hannah Arendt remarks, “[a]ll the European words for 'labor,' the Latin and English labor, the Greek ponos, the French travail, the German Arbeit, signify pain and effort and are also used for the pangs of birth. Labor has the same etymological root as labare ('to stumble under a burden'); ponos and Arbeit have the same etymological roots as 'poverty' (penia in Greek and Armut in German)” (1998, 48; note 39). 
the immortal soul, while work subdues the flesh and makes for self-discipline and application" (Gurevich 1985, 161).

This was especially true for farm labor because other forms of activity did not enjoy such approval. For example, synodal statutes forbade monks to take up such professions as weaving, cloth-making, shoe-making, dyeing, milling, smithery, and brewing, i.e. typically craftsmanship-like forms of activity. At the same time, it was assumed that intellectual work - to which the clergy was chiefly devoted - does not demand material remuneration: "in particular, teachers did not receive salaries, for wisdom was accounted as a gift of God which therefore could not be the object of mercenary trading. At best, a teacher could be offered gifts in gratitude for his dispensation of knowledge" (266).

The rapid growth of cities at the beginning of the eleventh century - simultaneously the effect and one of the main reasons of economic growth - evidently disrupted the above coherent image of reality. Little wonder then that it initially met with strong opposition. "According to the Bible, Cain was the builder of the first city. An English sermon of the fourteenth century contrasts priests, the knights and the workers, created by God, with the townspeople and the moneylenders, the spawn of the devil" (272). With the course of time, however, the Church adapted, albeit reluctantly, to the changing reality, both in practical terms and in the preached vision of reality. The same Bible from which the inferiority of labor was derived became the source of the theological idea "that Adam tilled the Garden of Eden, and hence, before becoming a form of penitence, labor had been an activity enjoying God's blessing" (265). Figures of craftsmen began to appear in church iconography, along with the spreading belief that all professions are worthy of a Christian, while all people have their vocations, the finding of which shall save them.

Well, perhaps not all professions, particularly not that of a usurer. "With virtually no excuses available, the usurer remained, during the thirteenth century, one of the few men whose trade was condemned secundum se, 'in itself', de natura, "by its very nature.' He shared this unhappy fate with prostitutes and acrobats" (Le Goff 1990, 50). Condemnation of usurers is related to the danger posed to old Christian values by the spreading money economy. Since its very beginnings, Christianity has propagated a cult of poverty and opposed the cult of Mammon, i.e. growing wealthy just for the sake of doing so. Already in middle of the thirteenth century, Thomas Aquinas stated emphatically: "Work has four aims. First and foremost, it must provide necessities of life, secondly it must chase away idleness which is the source of many evils, thirdly it must restrain concupiscence by mortifying the body, fourthly it allows one to give alms" (after Le Goff 1992, 222). 
The aim of the medieval economy was to secure survival, not to facilitate becoming wealthier - satisfying nessitas, not stimulating development. "Necessity legitimized work and even brought with it exemptions from certain religious rules"; however, "[a]ll economic calculation which went beyond providing necessities was severely condemned" (Le Goff 1992, 222-223). As Le Goff convincingly argues, economic growth, which was observed in medieval Europe in the eleventh and thirteenth centuries, did not stem by any means from the activation of certain developmental factors, but was primarily related to demographic growth, which entailed the necessity to feed, clothe, and shelter a growing number of people. The improvement of tools and modes of production was only an indirect consequence of that necessity. Surpluses were either hoarded or used to stimulate consumption, which - contrary to the "ideology of poverty" preached by the Church - was related not only to the nobility and the bourgeoisie, but also to the clergy. "It was normal that this indifference and even this hostility to economic growth should be reflected in the monetary economy sector and should put up strong resistance to the development within this sector of a spirit of profit of precapitalist type" (224).

The medieval substitute for the above "spirit of profit" was cupidity. "In the later Middle Ages," writes Johan Huizinga, "[...] cupidity becomes the predominant sin" $(1987,26)$. It was an epoch of poverty, which made people even greedier, and led to stronger condemnation of cupidity among those who fell victim to those possessed by this vice. "A furious chorus of invectives against cupidity and avarice rises up everywhere from the literature of that period. Preachers, moralists, satirical writers, chroniclers, and poets speak with one voice" (27). It is precisely excessive avarice that lays at the foundation of condemnation of usurers. However, even official texts would not refer to all involved with such business, but only to those "carrying on excessive usury" (Le Goff 1990, 72; emphasis preserved). It was also the combination of avarice, primitive pride and ostentatious boasting of one's riches that provoked greatest outrage. "Riches have not acquired the spectral impalpability which capitalism, founded on credit, will give them later" (Huizinga 1987, 26).

Before that could happen, however, until the end of the Middle Ages land and spoils of war remained the main source of wealth. Even the prosperity of medieval cities - despite their economy being based on trade and craftsmanship ${ }^{282}$ - was

282 Still in the early years of the fourteenth century trade involved mainly rare goods exotic and luxurious ones - making the group of their recipients limited to the upper classes, which were not really sizeable and whose wealth was additionally conditioned 
primarily rooted in land. "Even the towns which had most enriched themselves by trade $[\ldots]$ had to base their activity and power on their rural hinterland" (Le Goff 1992, 296). "The Autumn of the Middle Ages" in the fourteenth and the fifteenth centuries differed in many respects from the epoch's early years, but these differences are - at least in the social sphere - often a mere intensification of features typical for earlier periods, which later became more prominent. "The image of society would remain the same, or even revisit older concepts: in a society of three orders or estates nobility and clergy would still rule, despite the bourgeoisie becoming bigger, richer and more confident. It would be satisfied either by penetrating into the upper classes through ennoblement or by representing the entirety of the third estate. [...] In fact, these were times of 'feudal reaction': 'secondary serfdom' would emerge in the east and peasants would be even more tyrannized in the west" (Le Goff 1970, 360).

This necessarily telegraphic survey of transformations of labor across the period of around 7,000 years - i.e. from the rise of first city-states in ancient Sumer to what we assume was the beginning of the "capitalist era" - allows nevertheless to formulate several more general conclusions related directly to labor's role in shaping that form of sociality which we call traditional society.

First, it needs to be emphasized that, in this type of society, the division into those who work and those who do not is based on the division of tasks: those who did not work were distinguished not only (and not even primarily) by the fact that they did not have to work (i.e. did not have to personally undertake any activities to obtain food) but also because they performed a different role in the organization of tasks in the entire society. This is best visible in the case of feudal society, which explicitly stressed that all estates are complementary and form an organic whole. However, the division of tasks also functioned in Greek society - if we do not wish to follow Greek intellectuals in their attempts to exclude slaves and women from it, its differentiation would have to be described precisely in terms of the division of tasks. The specific character of that differentiation (which also appears in ancient empires and in the Roman Empire) lies in the fact that one social group - peasants or slaves, or anyone in between - would

by fluctuating income from agriculture that depended on atmospheric conditions. The same was true for craftsmanship, which also catered for the needs of the upper classes. 
be obliged, as it were, to keep others "alive" so that the latter can effectively perform their tasks.

Second, the particular content of these tasks was obviously defined by cultural parameters, but in all traditional societies the division of tasks among specific social groups was justified not by economic matters but by referring to the religious "order of the world" (or a political one, though rooted in religion). In this configuration, labor would appear as part of a divine plan, a kind of - one might say - "calling" of a particular social group, which the individual inherits through the sole fact of being born in it, the same as others inherit other tasks by being born in other groups. ${ }^{283}$

Third, labor is related mainly to the production of food. The organization of this "production process" differs among various kinds of traditional societies; however, since the fundamental "workplace" was land, it would invariably lead to the segmentation of society into relatively independent territorial units, and not functional ones. ${ }^{284}$ Each of those units constitutes a miniature of the entire society, embracing the typical triad of "lord, peasant, and parson," or counterparts to these categories found in other traditional societies. It is also within those territorial units that there emerge social bonds characteristic for a "traditional" type of sociality, and that labor is potentially divided into more specialized activities, including the production of goods other than food.

Fourth, territorially distinct societies are basically self-sufficient ${ }^{285}$ in terms of their functioning. By the same token, they are the ones creating a variant of base communities for traditional societies. Social integration occurs in them on the politico-religious level. We deal with the issue in greater detail in subsequent chapters. Let us only recall here that in the case of the feudal society the goal of

283 The calling to become part of a specific group can also occur through co-optation. Let us note that the concept of "the calling" has survived to this day among priests or politicians.

284 The term "segmentation" was coined by Durkheim (2013), who identified this kind of society primarily with clan-like organization of communities, although he also demonstrates that it survived until feudalism.

285 This also relates to medieval cities which - as we have already mentioned - had their own hinterland. At the same time, however, one needs to remember that their self-sufficiency depended to a large extent on natural disasters, foreign invasions, and epidemics - factors that would deplete the resources of the basic "means of production" in traditional societies, namely peasants. 
that level was to break those base communities and subordinate their population to another kind of "communality" rather than integrate them into a higher kind of social organism.

Fifth, a specific form of such territorial units are cities, which developed in the feudal society since the eleventh century. What differs them from the cities that existed in earlier forms of traditional society is primarily the fact that whereas the latter were, for the most part, centers of political life and large-scale trading, controlled by the group of the highest status, which made extra profit there (and sometimes the bulk of it), the former, i.e. mediaeval cities, brought to life a separate "task team" focused on exchange, which, in turn, would become the aforementioned "third estate" representing - as Le Goff indicates $(1992,260)$ - not the entirety of the bourgeoisie but only its wealthiest or best-educated part. ${ }^{286}$

The Protestant ethos that emerged towards the end of the fifteenth century is basically an expression of the period's twilight - a time when religion played the role of the fundamental factor integrating the fragmented image of reality, although (as Weber rightly observes) its emphasis on individual responsibility also heralds the later period when the character of social praxis, by foregrounding individual interest, seems to leave no room for the emergence of a collective "We."287 Weber's "disenchantment of the world" is, after all, nothing else than historical mothballing of such traditionalistic sentiments as faithfulness or gratitude, which lie at the foundation of a sense of community. It was no longer the community that was to assign tasks to individuals and social groups, but rather the rationally planned tasks began to determine the "community" of the company, bureaucratic organization or army. The shift from "calling" to "profession" was a move from norms determined by a moral universe to norms determined by the logic of utility.

\subsection{Denaturalization of labor - modern society}

In terms of all its features related to labor, the modern society that emerged in the nineteenth century stands in stark contrast to the traditional one. First, the

286 It was the revolt of the "third estate" many centuries later that put a symbolic end to traditional society, establishing the modern one.

287 Let us draw attention here to the fact that it was the persecution during CounterReformation that gave the Protestant bourgeoisie the character of a community not only in religious but also in secular terms, just like in the case of early Christian communes. The ethos itself does not seem to feature any elements establishing the necessity for individuals to cooperate in the realm of praxis. 
division into those who work and those who do not would be no longer based on tasks but on the criterion of employment. In this system, those who do not work include, on the one hand, the rentier class and owners of companies, while on the other: women performing household tasks, who are commonly regarded as "unemployed," and the new category of those who are capable of, and wish to, work but have no available jobs to take up. ${ }^{288}$ Second, the period saw the rise of real division of labor. All the activities related to the sustenance of human existence underwent far-reaching specialization subordinated to economic considerations; thus, labor became employment. Third, among those activities the production of food was moved into the background, while industrial production took the lead, with the administrative sector and services developing as well. Fourth, city became the crucial spatial form of population concentration ${ }^{289}$ yet simultaneously ceased to serve the function of a base community. Fifth, neither urban nor countryside areas (nor any other somehow distinguished social group) would be self-sufficient any more. A complex web of dependencies began to link all forms of spatial and social aggregations of population.

Naturally, none of these changes occurred on a one-off basis - the transition from the traditional society to the modern one took as many as four centuries, even in countries where the above transformations concluded first. The question about what started this process is one that has kept recurring in social sciences since the nineteenth century, when the magnitude of these changes was first acknowledged. Most theorists have focused on one, singled-out fundamental factor explaining the entire metamorphosis. For Marx, it was the development of production means; for Durkheim - the increasing division of labor; for Weber the Protestant ethos; for Sombart - the rationalization of economic activities, leading to the development of bookkeeping, calculation and modern enterprise in Italian cities. Certainly, each of those factors played a prominent role in the changes that occurred between the fifteenth and eighteenth centuries. However, one could also indicate that each of them did appear before without triggering such a thoroughgoing transformation. The development of production means, just like the increasing division of labor, has accompanied humanity since the very beginning. Asceticism through labor constituted one of the fundamental

288 Of course, during the Middle Ages there were also poor people who did not work, but in most cases they were incapacitated by age or disability. In modern society, the unemployed have an entirely different status, which is best expressed in the concept of a "reserve army of labor" introduced by Karl Marx. Cf. also Bauman 1998a.

289 Alongside processes of industrialization, processes of urbanization are considered to be crucial in the emergence of modern society. 
components shaping the Christian culture of monastic orders during the Middle Ages (Le Goff 1992). Bookkeeping and banking, which go hand in hand with economic activities, were already developed in the city-states of Mesopotamia. ${ }^{290}$ Finally, the period of greatest intensification of urbanization processes in Europe occurred between the thirteenth and the fourteenth centuries when a record number of cities were founded (Braudel 1992). All of this, however, did not erode the traditional form of sociality, which determined, for seven thousand years, the basic modes of institutionalizing labor and the dominant forms of social bonds emerging from them (in some parts of the world, the traditional form survives to this day). One could ask, on a similar basis, why it was England that became the hotbed of the new kind of rationality ${ }^{291}$ that lay at the foundation of the new, modern form of sociality - not Italy, which led the way as far as development of banking is concerned; not Germany, where Protestantism was born; and finally, not France or Switzerland, where inventiveness blossomed, leading to the construction of elaborate, automatic toys (Bronowski 1979).

Therefore, we return to the question posed in Chapter Three where we draw attention to the fact that the mode in which the human mind - and consequently, human behavior - functions is conditioned, among other things, by the dominant form of sociality, which selects the contents created and processed by the mind, not only ideas but also new technical solutions or behavioral strategies, as well as actions undertaken by individuals. In relation to the problems considered here, it follows that any specific effects of the above factors, which are ascribed a special role in the rise of capitalism, could only transpire after abandoning the form of sociality proper for traditional societies. This means, in turn, that the emergence of capitalism cannot be regarded - as would be suggested by theories discussed before - as an evolutionary process of economic development, within which people, while perfecting their tools, adapt to new means of production in a learning process, while new institutions are born out of the old ones. The rise

290 The term "Babylonian capitalism" does in fact appear in the history of economy. Cf. Wojnarski 2004.

291 Ferdinand Braudel's analyzes of the period before the fifteenth century demonstrate how much the modern rationality, which makes labor the basis of individual success, differs from the rationality of traditional society: "Potentially a pauper was the man who barely scraped a living from his work. If he lost his physical strength, if a marriage partner died, if there were too many children, if the price of bread was too high or the winter harsher than usual; if employers refused work, or wages fell - the victim would have to appeal for help to survive until better days. If urban charity provided for him, he was as good as saved. Pauperdom was still a condition in society" $(1983,506)$. 
of capitalism had to be preceded by a radical break away from earlier dominant forms of socializing human actions, and by the emergence of such factors that would entail a profound restructuring of the psychic apparatus of individuals as well as the social bonds which link them with others.

Robert Brenner (1989) reaches a similar conclusion, when he analyzes the premises of the belief that economic growth is a "natural" effect of human orientation towards the increase of profit. He draws attention to the fact that assuming the idea of the "invisible hand of the market," which exploits this motive of human action, entails making a series of unarticulated assumptions that have no justification in historical knowledge - assumptions about what is rational and what is possible from the perspective of social actors. As he demonstrates, until feudal peasants possessed land they could inherit from generation to generation, the crops providing at least a minimum on which to survive, and until lords had at their disposal all means of coercion, which allowed them to make a living from rents paid in one form or another by the peasants, the lords' rationality would rather incline them to invest in the arming of troops which allowed them to both keep peasants in line and wage wars in order to extend their territories, whereas the peasants' rationality inclined them, at best, to rebel or flee when rents soared high enough to endanger their and their families' survival. In other words, the rationality of individual behavior was a derivative of the internal logic of the dominant form of socializing the modes of producing means necessary to reproduce the feudal society in its biological dimension.

Thus, in Brenner's view, the basic factor enforcing changes to the rationality of survival and expansion was the emergence of a large segment of the feudal society which did not have any guaranteed access to land and was therefore deprived of certainty about sustaining its biological reproduction. ${ }^{292}$ As he shows, this occurred earliest in England, where enclosures and the process of shifting to land lease began already in the thirteenth century, which led to the situation in which individual tenants were forced to compete with each other for access to land. Naturally, it was not the direct aim of English lords to introduce new ownership relations - they only wanted to multiply their profits by taking advantage of the demand for wool. Thus, they adopted means proper for the rationality of the period, i.e. began violently grabbing commons and the land that belonged

292 Before Brenner, a similar thesis was formulated by Karl Polanyi (2001), who indicates that the basic factor that initiated the "great transformation" of an agrarian economy into an industrial one was the separation of laborers from means necessary to sustain life. 
to peasants. Similarly, the peasants-turned-tenants did not immediately subordinate their actions to maximization of profits. In the fifteenth century, tenancy rates were still relatively low and did not necessitate abandoning the rationality of survival. However, "as the demand for land, for food, and for raw materials thus began to grow in consequence of the expansion of population, commerce, and industrial production in the course of the sixteenth century, tenants found themselves under pressure to maintain their holdings"; they had no other choice but to adapt their production to the rationality of profit-making, "for unless they specialized, accumulated, and improved, they would fail to compete successfully on the market for leases and lose their plots" $(1989,49)$.

According to Brenner, it was the specificity of solutions adopted by English feudal lords to multiply profits from land that explains both why capitalism developed earliest in England, and why Central European countries would gradually fall behind economically - countries where the same goal was realized through a re-introduction of serfdom, thereby postponing for a long time any transformations of rationality lying at the foundation of economic activity. At the same time, it is crucial that the shift away from the rationality of survival to one of profit-making did not entail any fundamental change in the basic motives driving people's behavior. Adam Smith's claim that it is self-interest that acts as this basis could be probably true for any form of sociality. The issue is rather that this self-interest was put in the service of innovativeness and calculated striving for profit, no longer helping with survival wedded to unreflective greed (as Huizinga bluntly puts it) or the will to amass riches.

While accepting Brenner's thesis, ${ }^{293}$ we need to remember about two important factors accompanying the discussed transformations of rationality, especially in the early stages of this process. First, the elevation of self-interest to the rank of a chief principle in individual actions - an interest subordinated to the rationality of profit-making - did not create any new forms of sociality despite necessitating the destruction of those that dominated in the past. As we have indicated before, the form of sociality is established on the basis of emotional ties, which entail obligations to a larger community, whereas being driven by profit-making demands rational calculation, which does not allow considering

293 Let us draw attention to the fact that this thesis perfectly fits all theories which claim that capitalism began with the development of cities. After all, from their beginnings, cities have concentrated people whose majority would have neither direct access to land, nor would be able to forcefully appropriate crops. At the same time, the discussed thesis offers an explanation of processes in which "urban mentality" could become widespread and permeate into other segments of society. 
any other motives than economic ones. Second, calculation is an option available to those who have some, even slightest means, and believe that these means could be multiplied through hard labor and "a worldly asceticism." It would be difficult to convince poor people who live on parish support, alms or casual labor to adopt rational calculation or begin thinking in terms of profit. ${ }^{294}$ As Zygmunt Bauman writes, "the premodern poor - docile, reconciled with their fate, and not believing in the possibility of changing it - remained oblivious to the promise of regular pay in exchange for submitting to factory discipline. They lived like before, as far as anyone can remember: from day to day, and in submission to acquired habits. As long as they were able to eat as much as they did the day before, which is what they have grown used to, they would see no reason to make any effort to shake off poverty once and for all" (1998b, 5).

When combined, both these factors led to a truly inconceivable exploitation of workforce, which, at the first stages, comprised mainly of poor people fleeing to cities from rural poverty. In a traditional society, "the presence of people demanding consolation and support was both a challenge and a divine gift for all others. It was the necessary component in a society where earthly life derived its meaning from the eternal one, and where mortal existence was merely a preparation for the Last Judgement" (4). In the modern society that was budding in England, withdrawing from traditional forms of helping the poor was facilitated not only by the adoption of Protestant work ethics among the middle classes, which left little room for practicing the traditional Christian virtue of charity, but also by entirely practical reasons. The rapidly developing workshops, and later on factories, demanded more and more hands to work, which could be obtained - easily and cheaply - by gathering paupers inside them. ${ }^{295}$

Shortage of workforce was one of the main maladies of the growing capitalism. One of its nineteenth-century ideologues, Andrew Ure, argued that the

294 According to estimates quoted by Braudel $(1983,469)$, in Stuart England one fourth or even a half of the population lived below or near the threshold of poverty defined by the sum equal to half of income spent on bread in a family.

295 Workshops would be often set up in shelters, barracks, and prisons, which became centers of forced labor. For example, a spinning mill was founded in a prison combined with mental institution and orphanage in Pforzheim in the seventeenth century. Its workers would also include vagrants and beggars detained in the city. Similar workshops were organized in places such as Nuremberg and Basle. One well-known poorhouse in Paris (founded in 1656) housed 560 girls forced to labor, who were spinning, weaving, sewing and embroidering under the supervision of qualified craftsmen (Maciejewski 2001, 56). 
goal should be "to supersede human labor altogether, or to diminish its cost by substituting the industry of women and children for that of men; or that of ordinary laborers for trained artisans" (after Anthony 1977, 58). It was not only the cost that played the key role, but also the ease of training young people and those without habits formed by earlier labor experience, so as to adapt them to the requirements enforced by labor in the rapidly developing industry. ${ }^{296}$

These requirements were completely different from those that accompanied labor in traditional societies. The kind of labor that lies at the foundation of modern society was entirely denaturalized. This stems mainly from the fact that it ceased to be directly related to activities aiming at the sustenance of human existence. By referring to the terms proposed by Hannah Arendt, one could say more generally that labor was simultaneously clearly separated from work and subordinated to it. The former process finds expression primarily in the increased significance of the division between the countryside and the city, each focusing on different tasks. The latter process, in turn, is visible in the division into household and professional work. The importance of the last distinction is emphasized by Ulrich Beck, who writes that " $[t]$ he ascription of the gender characters is the basis of the industrial society, and not some traditional relic that could be easily dispensed with. Without the separation of male and female roles [...] there would be no bourgeois society with its typical pattern of work and life" (1992, 104; emphasis preserved). ${ }^{297}$ Sustenance of "life" within a household ceased to be related to labor, as everyday activities of women were considered something obvious and unprofitable. It is the man who became the family's only "breadwinner" - and it is his activity, related to participation in production processes, that began to be called labor. As Toffler underlines (1989), for the first time in human history production and consumption became separated.

Denaturalization of labor also stems from the fact that it ceased to be tied to the natural cycle of life, in which periods of activity are interwoven - both in daily and yearly rhythms - with periods of rest. It would be no longer a task whose goals are obvious as such because they are derived from the human condition (one needs to eat, defend oneself, as well as earn the favor of God or gods), but a dismembered set of activities whose ultimate outcome or goal cannot be figured out at all. Labor ceased to mean using tools, which constitute a natural

296 In the 1830s, the number of adults employed in the cotton industry would still amount to only 25-28 per cent of all employed (Maciejewski 2001, 109).

297 In the terminology we have adopted from Arendt this distinction is one between labor and work. 
extension of the hand; instead, it started to involve the operation of ever more complex machines and equipment, in which one careless move might carry huge consequences (e.g. breaking of the machine, disability or even death). This is related not only to physical labor but also to the so-called intellectual work in bureaucratic institutions that developed parallel to industry.

One consequence of the denaturalization of labor was the rising significance of disciplining human body in modern society, along with surveillance of its functions. As Michel Foucault writes, "[m]any disciplinary methods had long been in existence - in monasteries, armies, workshops. But in the course of the seventeenth and eighteenth centuries the disciplines became general formulas of domination. [...] Discipline increases the forces of the body (in economic terms of utility) and diminishes these same forces (in political terms of obedience). [...] If economic exploitation separates the force and the product of labor, let us say that disciplinary coercion establishes in the body the constricting link between an increased aptitude and an increased domination" (1979, 137-138). Disciplining occurs by way of disassembling the collective into individuals, whole activities into isolated gestures, and products into their constituent parts - in order to reassemble them in a new, scientifically grounded way that guarantees better organization of a given activity and increased efficiency.

Adaptation to the mode of labor characteristic of industrial society occurred primarily in schools. Alvin Toffler draws attention to the fact that the overt program of mass education was only camouflaging the hidden one, which had a far greater significance for the organization of modern society than learning to read and write, or acquiring basics of history. "It consisted - and still does in most industrial nations - of three courses: one in punctuality, one in obedience, and in rote, repetitive work" $(1989,29)$. Learning follows the rhythm of the bell, and its scope is determined only by the teacher, who orders children to do oftrepeated exercises meant to develop skillful hands (writing) and linguistic proficiency (reading). ${ }^{298}$ Any deviations from norms imposed by the school would be

298 Foucault draws attention to the transformations of the organization of classes during the transition from Jesuit colleges to public schools in France during the Empire. In the former, classes could have even a couple hundred pupils, and would be divided into ten-pupil groups, ascribed to either the "Roman" or the "Carthaginian" camp. "The general form was that of war and rivalry; work, apprenticeship and classification were carried out in the form of the joust, through the confrontation of two armies; the contribution of each pupil was inscribed in this general duel; it contributed to the victory or the defeat of a whole camp" $(1979,146)$. Since the second half of the eighteenth century, classes in schools were already organized in accordance with today's 
punished (physically, by sitting at a separate table, or through grades and entries in record books).

While analyzing the sense of punishment in various nineteenth-century contexts, Foucault is right to stress that "the art of punishing, in the regime of disciplinary power, is aimed neither at expiation, nor even precisely at repression. [...] The perpetual penalty that traverses all points and supervises every instant in the disciplinary institutions compares, differentiates, homogenizes, excludes. In short, it normalizes" (1979, 182-183; emphasis preserved). This norm has a specific genesis since it does not arise from a total image of the world, in which a specific place would be ascribed to particular individuals or groups, but is a derivative of utility considered from the perspective of efficiency and effectiveness. "The order that the disciplinary punishments must enforce is of a mixed nature: it is an 'artificial' order, explicitly laid down by a law, a program, a set of regulations" (179). Thus, the social order arising from it has to be again "normalized" or, more precisely, "naturalized."

As we have already mentioned, the rise of capitalism was preceded by the destruction of the traditional form of socializing labor. This meant, among other things, the shattering of local communities as base communities, i.e. ones in which dominant social groups could meet and forge social bonds characteristic for the traditional society. In the slowly developing modern society it was the family that has initially taken their place. In the countryside, the family was (basically, since always) the basic unit of production. As Michelle Perrot and Anne Martin-Fugier point out, "[p]rotoindustrial development relied heavily on the family cell, on the coincidence of workplace and residence. [...] The small family business - store or workshop - is another French dream that has persisted stubbornly despite the vulnerability of small business to bankruptcy and the attendant risk of family dishonor" (1990, 116-17). At the beginning of the nineteenth century, most products in Europe would still come from workshops located in houses, where craftsmen and their families would rely on their own skills in processing of materials provided by tradesmen (Rosenberg 1986, 148). ${ }^{299}$

individualistic principle: every pupil would be responsible for him- or herself, and everyone would individually compete with others.

299 In order to protect their entitlements, weaving guilds forced the British Parliament to pass a special law in 1555, which allowed setting up workshops outside cities as long as they were equipped with no more than two looms and employed no more than two journeymen. In the eighteenth century, in some villages in Picardy and Artois in France, for every 100 households 90 would be entirely devoted to outwork. 
With the passage of time, and along with the increase in economy's complexity, which was accompanied by the introduction of greater mechanization of labor, workshops began to be replaced by factories. In France, they would be first located close to the countryside so as to allow employing all members of the family. The "family team," directed by the father and including mother and children, provided the solution to the problem of supervision. "The mill owner himself set the example. He lived near the plant, sometimes on the factory grounds. His wife kept the books, and he invited employees to share in family occasions. Paternalism was the first system of industrial relations. Its benefits flowed to a core group of key workers whose reliability was essential. The system was feasible only where willing workers resided near the workplace. Paternalistic industrialism spoke the language of the family [...]" (Perrot \& Martin-Fugier 1990, 118). One could say that whereas in the outwork system family was an enterprise, later on enterprises would become an "extended family."

Relations within those "families" were never idyllic. Especially in England, company owners would act as "punishing" rather than "loving" fathers. Naturally, it was the pay that was the basic source of conflicts. In the first half of the nineteenth century, in their attempts to increase profits, employers would not pay their workers in cash but in products. Throughout the nineteenth century, the so-called iron law of pay remained in force, along with the belief in the existence of the so-called natural cost of labor, which was supposed to be equivalent to the goods and services necessary to regenerate the employees' capacity to labor (Daszkowski 2002, 17). In practice, this meant starvation-level rates. The awareness that wages exceeding the natural cost of labor could be an incentive to increase productivity among workers, and consequently increase the profits of company owners, developed among the latter very slowly. One true "revolutionary" in this respect turned out to be Henry Ford.

As Zygmunt Bauman rightly observed, by doubling one day his workers' wages, Ford "wanted to tie his employees to Ford enterprises once and for all, to make the money invested in their training and drill pay, and pay again, for the duration of the working lives of his workers. And to achieve such effect, he had to immobilize his staff [...]. He had to make them as dependent on employment in his factory $[\ldots]$ as he himself depended for his wealth and power on employing them" (2001a, 21). Tying employees to the company was not limited to increases in pay. In the course of time, entrepreneurs would start building entire housing estates for them, opening special shops, establishing mutual assistance funds, and developing programs meant to foster healthcare and provide pensions.

This modern variant of paternalism caused the company to become the basic living environment, not only for regular workers but to all the employed, 
including the owners. The certainty that employment at a particular company makes one tied to it for the rest of his or her professional life contributed to the development of what Bauman has called "long term mentality," which boils down "to an expectation born of experience, and by that experience convincingly and ever anew corroborated, that the respective fates of people who buy labor and people who sell it are closely and inseparably intertwined for a long time to come - in practical terms for ever - and that therefore working out a bearable mode of cohabitation is just as much 'in everybody's interest' as is the negotiation of the rules of neighborly fair play among the house-owners settled in the same estate" (2001a, 23). This bred loyalty, responsibility and even the readiness to make sacrifices.

This is precisely what "naturalizes" the company, turning it - or, to speak more broadly, any workplace since this mode of employment included also institutions based on hired labor other than the industrial ones - into a base community of the modern society. Labor in such places would be still organized artificially, i.e. differently than activities undertaken in private life, but thanks to school training, which would begin early and spare none, it became something obvious. Anyway, as Richard Sennett aptly remarks, "routine can demean, but it can also protect; routine can decompose labor, but it can also compose a life" $(1998,43)$. Thanks to the routine of social life, people can plan their individual biographies - it is more or less predictable, first, what course a particular life may take because despite the prevalence of meritocratic rules - inheriting social status would be still strong; and second, what pace shall mark one's career progress, due to the principle of seniority; and finally, what one might be doing after retiring because one's retirement pension became calculable in advance.

Of course, modern society is largely structured, but in the middle of the twentieth century the place of strongly hierarchized and thus conflictual class divisions from the first stages of capitalism came to be replaced by a more balanced social system based on a social contract between capital (employers), work (employees) and the state (government). The state began to be engaged in public investments (e.g. construction of motorways, or urbanization) in order to support mass production and consumption, which, in turn, was supposed to guarantee almost permanent employment. The state would also oversee public spending (better education, healthcare and pensions), providing citizens with a relatively fair living standard, thus encouraging consumption. The legal framework regulating those relations, as well as strong institutions, facilitated stable economic and social growth. Trade unions would become stronger. With better organization, they could often wield considerable political power. Their preferences were reflected not only in labor law but also in social policy, making 
them the architect and main proponent of the post-war welfare state, which strove for full employment, social security and decent pay.

However, as Zygmunt Bauman aptly points out, the concept of welfare state originated not with the desire to grant everyone fair living conditions, but with the necessity to support the unemployed, who would form a reserve army of labor "on standby." Welfare state "was a contraption meant to tackle the anomalies, prevent departures from the norm and defuse the consequences of norm-breaking were it to happen, nevertheless. The norm itself, hardly put in question, was the direct, face-to-face, mutual engagement of capital and labor, and the solving of all the important and vexing social issues within the frame of such engagement" (2001a, 22). In other words, it was a more or less peaceful coexistence that served as the norm, arising from the awareness of interdependency. The basic indicator of the effectiveness of this form of sociality was the numerical growth of the so-called middle class.

\subsection{Dematerialization of labor in contemporary society}

Routine, which constitutes the basis of labor in modern society, reduced the division of labor to the categorization of actions, and in the case of unskilled labor even to isolated gestures. The assembly line became the symbol of modern society. However, it is important to remember that routine also characterized the work of state or municipal officials and other white-collar workers. Weber's bureaucratic organization that lay at the foundation of modern management of institutions was supposed to rationalize the use of time, which demanded the use of routinized procedures. Changes that occurred over time were either integrated into existing procedures or sparked the development of new ones.

The stabilized world of day-to-day routines began to crumble in the $1980 \mathrm{~s}$ when certain changes in labor began to be acutely felt, although their origins went back to much earlier periods. Labor ceased to be a well-marked career path, which gave meaning to individual biographies, and began to resemble a rollercoaster ride full of ups and downs, sudden turns, unexpected events, and entailing many risks. Companies began to dismiss employees, move them to new posts, or suddenly demand travelling to the other side of the world. Large industry began to relocate factories to third-world countries, where workforce is much cheaper, while companies of long-standing tradition would bankrupt overnight. Unemployment rose, while benefits dwindled. Toffler's "third wave" of civilization-scale changes burst into people's lives with a dizzying force.

When considering the basis of currently experienced changes, it is usually assumed that they have been caused by the transformation of those 
production processes that are related to the introduction of new technologies and methods of management, necessitated after the Second World War both by the incessant contest between communist East and capitalist West, and the recession of the early 1970s, which brought the growth of the Western economy to a temporary halt. Certainly, these factors played a significant role, but one also needs to remember that the development of production depends primarily on a sales market, which is correlated with demand. Henry Ford understood this well when he decided on the mass production of his cars, expecting that the relatively high wages he paid to his workers would come back to him as soon as those workers begin to buy the cars. "The Fordist economy," Steven Miles observes, "was dependent upon the fact that workers had a surplus of disposable income which they could invest in the increasing diversity of goods that were being made available to them. This was a crucial development" $(2006,7)$. This, however, required time. As Miles makes it clear, "it was not until the 1950s that the accessibility of consumer goods began to transcend social classes and that the status-conferring qualities discussed decades earlier by Thorstein Veblen [...] came to have a more general social relevance" (8). From this perspective, the introduction of the credit card in 1950 was as momentous as the earlier invention of the steam engine. The industrial revolution gave way to the consumerist revolution, while the ethos of work was replaced by the ethos of consumerism, especially in its function of shaping lifestyles.

One could say that whereas in modernity people worked in order to earn means for satisfying their needs, the arrival of the consumerist era has made people work in order to satisfy their desires, which are "fueled" by the elaborate advertising "industry." As Claus Offe rightly observed already in 1985, labor has been ousted from the center to the margins of individual life. ${ }^{300}$ Thus, it has become a subordinate element of individual biographies. Lunt and Livingstone phrase this even more forcefully when they argue that "involvement with material culture is such that mass consumption infiltrates everyday life not only at the levels of economic processes, social activities and household structures, but also at the level of meaningful psychological experience - affecting the construction of identities, the formation of relationships, the framing of events" (after Miles 2006 , 9). Consumerism - or, to be more precise, its rejection - has also become

300 This is perfectly illustrated by a snippet of a telephone conversation the authors recently had during working hours. "What are you doing?" one of us asked and the other replied: "Nothing. I'm working." 
the main point of reference for mass social movements and therefore the fundamental field of contemporary political conflicts.

The subordination of the economy to consumerism was possible thanks to the aforementioned technological development, which allowed for a more flexible use of machines, swift and cheap production of short series of high-quality products, and quick reaction to market trends. This fostered a move from mass production to a model characterized by "diversified quality" (Lash \& Urry 1994, 72-73), i.e. oriented towards the creation of individualized products adapted to suit the needs of various client groups and different micro markets. At the most general level of analyzes, the entirety of the processes transforming contemporary economy is often called with the overall term of a transition from organized to disorganized capitalism (Lash \& Urry 1993). Whereas "organized capitalism" was distinguished by concentration and centralization of capital on regulated national markets, "disorganized capitalism" is characterized by spatial dispersion and decentralization of economic relations, freeing them from the supervision of the national state. This transition was accompanied by qualitative changes of the very nature of labor and its organizational structure.

The essence of these changes is probably best captured by the concept of "the dematerialization of labor," which is a derivative of "the dematerialization of economy" (Goldfinger 1998, 109). The term underscores the fact that in contemporary economies labor is increasingly less often organized around the production of objects (although they still constitute a significant portion of final products), and more and more often deals with ideas and abstract things. "A dematerialized economy transforms the nature of labor and its place in space and time. Labor is becoming dematerialized, both in terms of the objects produced, and the relations between employees and their environment (including interactions among people). What is more, the change of the nature of labor and its modes is accompanied by a deep transformation of the relation between labor and non-labor activities. Traditional boundaries between the two are becoming blurred, obscuring the division between the time and space devoted to labor and those related to what people do beyond it. Labor leaves factories and offices, appropriating private space and public spaces. Once so clear, the distinction between workplace and other spheres of life, and between time of labor and that of non-labor, is becoming increasingly fluid" (Chojnowska 2002, 2).

The phenomenon of the dematerialization of labor is usually associated with the shift towards the "knowledge-based economy," which would suggest the presence of a large intellectual load in processes of production. Certainly, adopting scientific achievements is a crucial feature of contemporary economy, but the above "dealing with ideas and abstract things" is definitely not related to 
scientific ideas. Rather, it is connected with the emergence of hosts of specialists whose basic task is, on the one hand, to improve labor's efficiency by adopting the best possible uses, not of physical power, but that of minds concentrated in an organization or working for it; and on the other hand, to improve the "efficiency of consumption," which primarily means, under conditions of intense competition, finding ever more complex ways of tying potential consumers with the products of given company. That is why the basic areas of the dematerialization of labor include the activities related, on the one hand, to the management of "human resources," and on the other - to marketing.

Management naturally encompasses various indirect goals such as increase of efficiency and flexibility, decrease of costs, etc. All of them, however, are related to an assumption which forms - as Richard Sennet claims - "the cornerstone of modern management practice," namely that "loose networks are more open to decisive reinvention than are pyramidal hierarchies such as ruled the Fordist era" $(1998,48)$. The network structure is supposed to be more flexible, better suited to the changeable nature of tasks faced by organizations in the era of disorganized capitalism, while teamwork is supposed to match "human nature," to which the routine of monotonously repeated activities is not only degrading but also wearisome.

In consequence, the two major fields of activity in management (of course, beyond running the company and making strategic decisions) include ceaseless re-engineering of organization, focusing on developing more effective ways of building networks of connections to decrease employment ${ }^{301}$ on the one hand, and on the other - organizing various workplace reunions and special trainings $^{302}$ that are not meant to develop strictly professional skills but rather foster better teamwork. ${ }^{303}$ After all, the last element demands sensitivity to

301 As Zygmunt Bauman puts it, today's large corporations list workforce among liabilities rather than assets: "today's slimmed-down, streamlined and flexible industrial plants - hungry for capital and knowledge - do their bookkeeping in such a way that employees are classified as liabilities, as a negative factor in calculations of productivity" (1998b, 5).

302 Naturally, each of those fields of activity has its own specialists; whereas direct running of the company is the responsibility of the management, all kinds of actions supposed to increase its efficiency are typically the domain of professionals employed by specialized companies.

303 As Sennett observed, there is basically no evidence that re-engineering increases the effectiveness of companies. Some even claim that it has the opposite effect, although the very fact of implementing it might increase the company's value as it testifies to changes that are valued in themselves. One could add here that trainings could 
others, the ability to listen, willingness to cooperate, orienting oneself quickly in new circumstances, and the aptitude to disregard any problems that could interfere with the joint accomplishment of specific tasks.

The second important area in which the dematerialization of labor has occurred is the rapidly developing marketing. Advertising as such constitutes only a small component of it, while direct appeals to potential customers to buy specific products are merely a distant echo of the strategies that currently assume much more complex and sophisticated forms. Contemporary marketing has come a long way from creating particular needs, through developing complete lifestyles, to the crafting of "life worlds," in which specific experiences or sensations are being sold as part of so-called experiential marketing. ${ }^{304}$ Today's marketing is an area of activities based on specialized knowledge not only about human actions but also about all those elements of mutual relations between humans and culture that facilitate the modelling of these actions in various ways. Nevertheless, they are always supposed to lead clients to the shelf that contains products of a specific brand.

Both management and marketing constitute an indispensable functional component of contemporary industrial organizations, which may still be oriented towards maximization of profits, but try to achieve that goal not through production but rather employment and sales. In this they are primarily aided by the "dematerialization of labor." Thus, one might say, with only slight exaggeration, that dematerialized labor is neither an activity aiming at the sustenance of human existence, nor one focused on production. Instead, it becomes "action" in Hannah Arendt's sense: a unique mode of engineering "souls" and interpersonal relations.

It is worth mentioning here that processes of the dematerialization of labor understood in this way are primarily related only to certain groups of people employed in contemporary economy, mainly to the "symbolic analysts" as Robert Reich calls them (1992). It would be difficult to speak of the actual dematerialization in the case of people whose job is to enter data into computers, or deal with clients in places where products or services are sold. After all, their activity

constitute an attempt at developing communal characteristics inside a formal organization, e.g. engagement, loyalty and mutual trust, which do not arise from real cooperation but are taught through exercises done in task teams, aiming to increase the effectiveness of teamwork.

304 Along the same lines, one may argue that "Nike towns" are a reworked version of Disneyland, which would also confirm the claim made by many that today's mass culture is covertly infantilized. 
has a more concrete and less abstract character, even though it does not lead to the creation of material objects. It is in the case of such individuals as well as other categories of people entering the job market that a particular significance is acquired by the transformations of the very structure of labor organization, which departs from the traditional division of workforce into the employed and the unemployed, the latter forming a reserve army of labor.

In contrast to the economy from the period of modern society, i.e. one based on long-term, often life-long and inherited relation with the company, today's economy is based on a flexible regime of employment, involving a gradable certainty of keeping jobs, which has led to the emergence of two, fundamentally different segments of the job market: the core and the periphery (Harvey 1995, $150-152)$. The core is a constantly dwindling group comprised of highly qualified employees who are essential from the perspective of long-term functioning of a given organization. Potential costs related to dismissing employees from the core favor the practice of subcontracting even at the highest level, which makes the core group relatively small. Its members are full-time employees, enjoying secure status and having opportunities to participate in trainings and become promoted. Moreover, they receive high salaries, social benefits, and other kinds of bonuses. In return, full availability is expected from them.

The periphery, on the other hand, includes two different subgroups. The first is comprised by full-time employees whose skills are easily obtainable on the market, e.g. salesmen, personal assistants or workers performing routine manual tasks - chiefly the so-called pink-collar sector. Members of this subgroup have little opportunity to pursue any career, and have smaller certainty of keeping their post since a large supply of such skills makes lay-offs among them relatively easy and natural. The second peripheral subgroup includes part-time employees, temporary workers hired on subcontracts, the self-employed, and those working in several places. They are so-called buffer workers whose employment security is even smaller than that of the representatives of the other group. They merely provide workforce depending on particular demand on the part of companies, often taking the so-called McJobs: unqualified, badly paid, insecure posts without any perspective of development, although this group also includes highly-qualified specialists hired to perform specific tasks. In both cases, this category of workers is not offered any trainings or promotion opportunities, and is not given any promises regarding continuation of work (cf. also Hartley et al 1991, 5).

The flexible system of employment thus assumes two kinds of flexibility. The core employees are supposed to be "functionally flexible:" in exchange for high salaries, security and good working conditions, they are demanded to do whatever the company currently needs them to do. At the same time, however, 
employees from the periphery of the job market are hired to perform specific tasks and are dismissed as soon as they are no longer necessary - in this case, we deal with "numerical flexibility." It is crucial that changes observed in the structure of the job market are parallel to those transformations that occur in contemporary economy's organizational structure. The economy itself is becoming increasingly polarized, with relatively few large, international corporations, on the one hand, and the other - countless small, often family-run companies, easy to found yet easily vanishing, which try to flexibly adapt to the conditions of ever-changing demand. ${ }^{305}$ Middle-size national companies have more and more problems with staying afloat, although they constituted the bulk of nineteenthand twentieth-century "mature capitalism" and simultaneously the social base of socio-political relations formed within that regime.

The above transformations of the nature of labor and its structural organization have two important consequences. The first one affects mainly independent experts as well as employees of those large corporations and state institutions that are being ceaselessly restructured. Endlessly moving from one project to another, their attitudes and work habits change in a direction that Richard Sennett (1998) sees as destructive, both from the individual perspective and from the perspective of the company's long-term interest. "The corrosion of character" involves individuals dropping such attitudes as loyalty, engagement, firmness, and being goal-oriented. They are hallmarks of long-term relations for which there is no place in the constantly changing workplace. For an average employee, working in such an environment entails being torn away from the past, lacking foothold or attachment to one's work, and most importantly - needing to accept its chaos and fragmentation, which prevents individuals from planning their lives. Their actions cease to be tied to the company's interest or good name, but begin to be oriented towards performing the role of an enthusiastic employee ready to accept any new challenge.

The second consequence produced by the shift to "disorganized" capitalism was the complete downfall of politics and institutions appealing to class interest. This is largely due to the disappearance of conditions favoring the development of trade unions as the main representative of working-class interests. Regardless of the reduction of that class's size, as Harvey notes, "struggling against capitalist exploitation in the factory is very different from struggling against a father

305 According to data quoted by Kazimierz Frieske, in 1999 the job market's portion belonging to small businesses amounted in the EU to 66 per cent, while in the candidate countries - to 72 per cent of all employed $(2004,365)$. 
or uncle who organizes family labor into a highly disciplined and competitive sweatshop that works to order for multinational capital" (1995, 153). However, the dwindling significance of the idea, according to which representing class interests ought to be the basis of actions taken in the public sphere, was also affected both by processes of globalized change in the role played by the national state in supervising economic activity, and - or perhaps even primarily - by the structural transformations of labor itself, which, in turn, led to such changes in social relations that have made it more difficult to define individual aspirations in terms of relatively stable interests.

The consequence was that - as Ulrich Beck put it - "the people have been removed from class commitments" $(1992,87)$. This leads to progressing individualization, which has been enforced by the structural properties of postmodern society. Although the process of individuals being "set free from the social forms of industrial society - class, stratification, family, gender status of men and women" (87; emphasis preserved) was in its initial stages largely alleviated thanks to the development of the capitalist welfare state, it currently also involves (perhaps crucially) the equaling of everyone's chance of becoming unemployed, with increasingly limited assistance provided by the state. "No qualification or membership in any professional group can protect one from unemployment," Beck strongly emphasizes. "What formerly used to be a fate shared by the entire group," he concludes, "is now [...] distributed crosswise among biographies [...] while a large portion of the population is at least 'temporarily' confronted with unemployment (and poverty)" (2002, 138 \& 141; emphasis preserved).

Labor has become a rare good. As Jeremy Rifkin writes in the symptomatically titled book The End of Work: "We are entering a new phase in world history - one in which fewer and fewer workers will be needed to produce the goods and services for the global population" (1995, xvi). Therefore, one might say that labor is being "dematerialized" also in the sense that, along with technological progress, the scope of actions for which humans are necessary becomes narrower. ${ }^{306}$ More and more people are sentenced to remain outside the world of labor. ${ }^{307}$ They no

306 As Ulrich Beck points out (1992), housework is basically the only area in which, until recently, there was a "technological gap" which necessitated human work. However, this area has been recently invaded by "processes of technical automation," which leads - according to Claus Offe (quoted by Beck) - to the "deskilling of housework" (110; emphasis preserved).

307 According to estimates of the International Labor Organization, almost a billion people worldwide are deprived of work or are forced to accept part-time employment. Taking into account that the number of people capable of working is estimated 
longer constitute a reserve army of labor, but are quickly becoming a dispensable part of a society (Bauman 1998a) in which the place of the individual is determined not by labor but by consumption.

"Glorification of hired labor as humanity's greatest calling, the condition of moral decency, a guarantee of law and order, and simultaneously a patented solution to poverty," Bauman writes, "harmonized with the once labor-hungry industry, in which the production output would grow along with the number of staff" (1998b). This sounds anachronistic today, not only because large corporations have adopted new major sources of profit, namely downsizing (reduction of employment by utilizing new technologies) and re-engineering of organizational structure, but also because it is consumption, and not labor, that has begun to provide meaning to human activities, and to act as the driving force behind market economy. However, the juxtaposition of these two reasons demonstrates unequivocally that although labor ceases to be one of the fundamental areas of socializing human actions - since it no longer produces any bonds, creating adaptations at best - it might soon become one of the most explosive areas of human activity. After all, it is difficult nowadays to be a consumer if one does not have a job.

to amount to ca 3 billion, this would mean that one in three people around the world are either unemployed or do not enjoy full-time employment (Budnikowski 2002, 7). 


\title{
CHAPTER SEVEN. TRANSFORMATIONS OF THE PUBLIC SPHERE
}

\author{
[...] the political realm rises directly out of acting \\ together, the "sharing of words and deeds."
}

HANNAH ARENDT, The Human Condition

Issues related to the public sphere certainly occupy a vital place in reflection on contemporary society, mainly because the term "public sphere" tends to be associated with questions of civic society, freedom of expression and political participation. Transformations of the public sphere are more or less directly related to the very heart of democracy. One could also note that - just like in the case of transformations occurring in the realms of family and work - this sphere also appears to be often diagnosed as facing crisis. This has been articulated, for example, by Seyla Benhabib, who writes: "Undoubtedly, our societies are undergoing tremendous transformations at the present. In western democracies, under the impact of corporatization, the mass media and the growth of businessstyle political associations, like PAC's (Political Action Committees) and other lobbying groups, the public sphere of democratic legitimacy has shrunk" $(1992,112)$.

The conviction that the public sphere is in crisis permeates the three main currents in philosophy of politics distinguished by Benhabib: first, the agonistic model of Hannah Arendt, which originates in the republican concept of civic virtues; second, Bruce Ackerman's model of public dialogue, which originates in the liberal tradition; and third, Jürgen Habermas' model of a discursive public sphere. Despite the fact that each of those currents defines the category of the public differently, it is easy to notice that all of them take a certain normative pattern as their point of departure. The noblest forms of such models would be invariably located somewhere in the past: in different periods of European history, depending on the current. Thus, the public sphere becomes incorporated into specific myths of the golden age, which are on equal footing with the myth of the lost primal community, the myth of a consolidated pre-industrial family, or the myth of work rooted in religious values, i.e. understood as a creative and meaning-making activity.

At least for that reason it could be said that the concept of the public sphere clearly features an additional component carrying value judgments. The existence 
of the public sphere - naturally, in the form close to the model assumed by a given author - would be implicitly regarded as something good and desirable. Therefore, any deliberations on the changing public sphere demand - already at the very onset - that this more or less decidedly identifiable normative reference be revoked. To an equal extent, however, they demand that we consider the very sense of the concept since it is far from unambiguous. This is expressed by the historian Philippe Ariès, one of the editors of the monumental study $A$ History of Private Life. In the introduction to the third volume, he confesses that he used the public/private distinction for many years in numerous analyzes, assuming that it would correspond to the distinction between the intimate (i.e. that which is hidden from the eyes of others) and that which is there for everyone to see, but remained unaware of a different possible approach. It has taken him a lot of time to recognize that some "conceived of the public/private dichotomy differently." As he notes, "a second aspect of the public/private opposition had escaped me because I have been alienated from political history. The word 'public' also refers to the state, and to service of the state, whereas 'private' (or particulier, as one used to say in French) referred to everything outside the state's purview. For me, this was a new and most rewarding way of looking at the question" $(1989,9)$.

These two approaches, or aspects of the public/private opposition, also appear in sociological discussions. It could seem that, in the case of analyzes focusing on the transformations of the public sphere, the simplest solution would be to clearly subscribe to one of them. This would make it possible to precisely demarcate the field of enquiry while avoiding accusations of disregarding another, equally important aspect of the issue. However, the very fact that double meanings of the public/private opposition do coexist, as noted above, can suggest that they may be linked in a way that could prove to be vital from the perspective developed here. Thus, let us first examine the various shades of the meaning of the two terms.

\subsection{Semantic aspects of the public/private opposition}

The public/private opposition appears already in classical Latin. The semantic core formed in this context survived throughout the Middle Ages - including the era of "barbarians" 308 - and was preserved in local languages. It can be traced in nineteenth-century dictionaries of French and English, and has survived to

308 We mean here the period of departure from Roman law in favor of common laws. 
this day, offering the basic semantic intuitions in European languages. In Cicero's Latin, the term privus denotes that which is specific (particular) and private; to act privatim means to be doing so at one's own place, at home ${ }^{309}$ - not as a magistratus (official) but as an ordinary citizen, i.e. on a different legal ground. Furthermore, privatum denotes one's own means, things for one's own use. ${ }^{310}$ In privatio refers to making something in isolation, at one's own home. Thus, the original sense of the term "private" refers not to the individual as an isolated unit but to a person belonging to a "house community," while the sense of the term "public" points to a higher-order collectivity, i.e. the political community of the polis, tribe or state. Res publica therefore encompasses that which is not the private property of any group and consequently cannot be legally traded or exchanged on the market. ${ }^{31}$

"The Latin of chroniclers and charters characterizes as publicus that which falls to the sovereign, which is part of his regalian rights or which falls within the jurisdiction of magistrates charged with preserving peace and justice. [...] The publicus was the agent of sovereign power, the persona publica, responsible for acting on behalf of the people to defend the rights of the community. As for the verb publicare, its meaning was to confiscate, seize, remove from private use or ownership" (Duby 1988, 5; emphasis added). The meaning of the term privatus broadened, encompassing not only that which belongs to the family, but also everything that lacks a festive character. For example, in the Rule of Saint Benedict, privatis diebus means "weekdays." "The notion of the festival will prove to be important in what follows. We shall be looking at ceremonies and staged spectacles, at the gestures that people made, the words they spoke, and the way they presented themselves to others. Words associated with privacy were not applied to this kind of festive behavior but reserved for more homely activities, especially those prescribed by rule for monks" (5). Such activities would have a specific function in a given community. In a monastery, monks would hold certain sets of prerogatives - rights exclusive to all members of the closed

309 It is important to remember that "home" denotes a separate legal territory, not the lack of law.

310 Hence, res privata can find itself in commercio and in patrimonio - i.e. it can become an object of trade, exchange, inheritance; res publica, on the other hand, remains extra commercium (outside trade) and cannot become an object of exchange.

311 Similar semantic intuitions can be found in the classical definition of the public good its essence would consist precisely in the following: everyone can use it and access to it cannot be restricted in any way. 
community separated from public life by means of monastic enclosure. All of its members enjoyed this privitas. Therefore, privitas denotes in this context everything that is happening within an isolated community regulated by its own rules of behavior.

Littrés nineteenth-century dictionary also points to home-related themes as constitutive elements of the original sense of the term privé: "private" would mean "tamed" or "domesticated," e.g. un oiseau privé refers to a domesticated bird. "Private" is therefore connected to ideas of familiarity, intimacy and attachment; it belongs to the group of concepts related to family, home, interior, but also confidentiality. After all, the word "private" also shares affinities with the adjectives "secluded," "secret" or "reserved."312 The scope of the term privé also includes the concept of a "particular person" (privé means particuler), to whom something may belong as his or her property, therefore being somehow "reserved" and made inaccessible to others. Two quotations from Littrés dictionary are particularly characteristic in the context of the public/private opposition: "those who rule make more mistakes than private people" and "nothing is private in the lives of great people as everything belongs to the public good." In turn, the concept of the "public" is defined in Littrés dictionary as "[t]hat which belongs to an entire people, that which concerns an entire people, that which emanates from the people" (Duby 1988, 3), i.e. that which is of common use things that are not only open and universally accessible, but also overt, visible, "for all to see."

As it becomes apparent from the analysis of the original meanings of the public/private opposition, these terms did not initially refer to the distinction between the individual and the state, but they referred to the distinction between two kinds of communities: the domestic, local one bound by friendship and tradition, and the political one tied by relations of power and law. What is more, within each community there existed a space where certain kinds of behavior would be made "common:" in the former, it would be shaped by particular habits or traditions, while in the latter - by universal law. In this original interpretation, the key issues are not related to the sole existence of the public sphere, but include the balance between the public and the private, their mutual relations, the mechanisms of making things "public" or "private," and finally, those spheres of life that are either lifted into the limelight from the realm of privacy or withdrawn into this realm and in time made intimate.

312 According to Littrés dictionary, "Private life should be lived behind walls" (after Duby 1988, 3). 


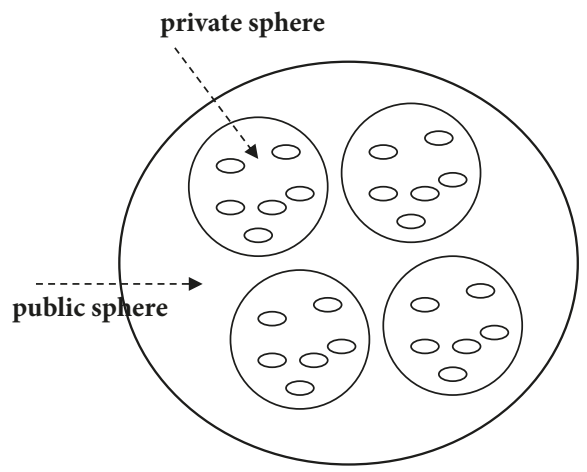

Fig. 7.1: Private and public sphere

At the same time, it needs to be emphasized that the terms "private sphere" and "public sphere" are not only relative but also invariably refer to communities located on different levels of the hierarchical organization of society. In the original sense, the "private sphere" would be related to the household community, while the "public" one - to that of the state, which would mean that the public sphere is nested inside a number of private ones that constitute, for those who partake in collective life, the primary common space. However, the household community or any other local, particular one - also contains a certain public sphere, in which individuals are "submerged." The difference between the level of household communities and the level of the political community would lie only in the following: in the original sense, the private sphere is the space of intra-group integration, whereas the public sphere is the space of inter-group integration.

One could therefore say that the public sphere is "the group of groups" - a space inside which the particular becomes the general. In this perspective, great significance is given to the principle of including "private" groups in the public sphere, which determines the balance between these two levels of integration. Theoretically speaking, the inter-group space can be occupied either by all members of these groups, or only their representatives. In the former case, boundaries of lower-order groups are dissolved and they are reduced to the narrow sphere of intimacy or mores. ${ }^{313}$ In the latter case,

313 This is the origin of controversies around parental rights regarding children: the more individual rights are gained by the latter, as guaranteed by public institutions, the less "private" the family becomes and the fewer rules operating inside it have a particular character derived from tradition or mores. 
certain members of the lower-order groups are excluded from participation in the public sphere. For example, it was fathers of families (patres familias) that constituted the Roman political community, while women, children and slaves remained outside the public sphere. Therefore, heads of large families acted as a bridge between the private and the public, ensuring the balance between the two realms and securing the stability of the entire complex social order. $^{314}$

The semantic tropes found in contemporary interpretations of the private/ public opposition initially seem to be entirely different. This stems from the fact that the authors of currently developed theoretical models ${ }^{315}$ focus primarily on the concept of the public sphere, often implicitly assuming that the private sphere constitutes the domain of individual people's actions and encompasses everything that is not related to others who belong to broader societal categories. Thus, in more general terms, it would remain an intimate matter of private preferences. Nevertheless, in these models there also invariably emerges the question of individuals being rooted in lower-order groups.

For Hannah Arendt (1998), the Greek polis or the Roman res publica, which she regards as the original model, are spaces where individuals can reveal their specific characteristics: one's moral greatness can not only be discerned and acknowledged by others but also become part of a shared tradition, setting up new standards of behavior and forging new values. "This is a competitive space," writes Benhabib, "in which one competes for recognition, precedence and acclaim" (1992, 93). At the same time, however, in Arendt's view the public sphere also has - as Benhabib calls it - an "associational" character: it can emerge in all places where people work together upon agreement or as a result of persuasion, and where centers of coordinated action are created.

According to the liberal model, in turn, the public sphere acquires a "legalistic" character. Benhabib defines it as a space of rights and principles, thanks to which "different primary groups, about whom we only know that they do not share the

314 The quoted example aptly illustrates not only the irremovably relational character of the pair private/public but also the fact that the rules determining transitions from one to the other constitute the keystones of socio-political order. The question of balance between levels of integration is also related to individual participants in social life. As Norbert Elias noted, "in the transition to new level of integration [...] there are conflicts of loyalty and conscience which are at the same time conflicts of personal identity" (2001, 179).

315 We employ here the typology developed by Seyla Benhabib (1992). 
same conception of the good, can 'resolve the problem of coexistence in a reasonable way" $(1992,96)$. However, such a "reasonable resolution" would require to exclude from public discourse those moral questions which - out of their very nature - cannot be settled; their place would be taken by accepted procedures or normative premises of public dialogue (e.g. the principle of equality), which "all political participants find reasonable (or, at least, not unreasonable)" (97).

It is worth noting, however, that in the liberal model - as underlined by Will Kymlicka (2002) - the public sphere is the realm where groups coexist, not individuals. Its primary function is to create an infrastructure facilitating constructive dialogue that would bring pragmatic profits. The question of a "good life" would be resolved in the private sphere, inside groups, while the public one would be the place for settling questions of justice and social choices understood as an optimum mode of aggregating preferences rather than a way of reaching consensus in social discourse. The public sphere would be thus a space in which, on the one hand, various groups can coexist peacefully, while on the other - a common direction for action is negotiated.

Finally, in the third model distinguished by Benhabib - one developed by Jürgen Habermas (1989) - the public sphere is conceived as a space located between the state apparatus and social groups. Inside it, reasonable discourse is possible without violence. While Arendt argued that it was precisely the development of a "social sphere" between the family and the state (a sphere of social interactions that mediate between the two) that caused the downfall of the public sphere, ${ }^{316}$ for Habermas its emergence is the very moment when the public sphere was born. According to him, its demise would be rather associated with the extension of the state's prerogatives. ${ }^{317}$

According to Habermas, the bourgeois public sphere, as distinct from its ancient predecessor, had a particular character: its participants would not act for the benefit of the political community to which they belonged, but for their

316 Whereas household communities or, to a lesser extent, local ones constitute a miniature of the political community, "groups of interest" assembled as a result of interactions occurring in the "social sphere" have a completely different character. In their case, the common good has a "club-like" rather than public character.

317 Strictly speaking, the crisis of the public sphere was caused by the political influence of larger social groups gained as a result of reforms extending the right to vote (introduced in Britain in 1867 and 1883). This reinforced the position of state because the poorer parts of society, which successfully campaigned to obtain compensation for unequal economic opportunities, have led to the development of interventionist policies. 
own profit. However, since they occupied the same social position, it was possible for them to reach agreement and establish goals whose fulfillment would be in everybody's interest. In Habermas' model, therefore, the public sphere's key components include the concept of equal status among members and the principle of rationality. The justification of postulated premises would consist in the process of formulating them as they need to be the outcome of a discussion based on the criterion of rationality, i.e. the principle that it is not the authority of power but objective rightness that determines their importance. It remains unclear, however, what would be the significance of the condition of members being rooted in private communities of local, family or religious character, which belong to a completely different order than the one related to social stratification.

Regardless of the differences between the three models, they all share the conviction that it is precisely the public sphere - where particular individuals and groups meet - that constitutes the essence of collective life because it provides a space in which crucial choices are made, ones that determine both the basic rules of social life on the inter-group level and the principles of peaceful coexistence, coordination and cooperation, creating a framework in which individuals can "reveal" themselves by emerging from the realm of private life. All of them are also informed by normative ideas such as the open character of the public sphere, the transparency of its organizing principles, the equality of those who participate in it (secured by suspending their private status in order to grant them a more general identity as citizens) and the rationality of discourses developing within it.

Despite their highly normative character, in each model one can find a jarring note, namely the question of excluding certain groups or issues from the public sphere. The problem disregarded by Hannah Arendt in her nostalgic praise of Greek democracy is that neither the Greek polis nor the Roman res publica were open to all. In fact, women, slaves and metics did not enjoy the status of citizens in them. As non-citizens, not only were they excluded from decision-making processes regarding the political community, but they were also subject to the laws governing the internal sphere of oikos, against which there was no appeal. In Arendt's perspective, the public sphere is invariably related to the question of participation and exclusion - the unconditional or solely conditional (licensed) openness of the space that lies outside the private sphere.

As for the liberal model, in turn, its criticism would focus on excluding certain moral issues from public dialogue, specifically ones related to the good life. They would be left to the private sphere, i.e. groups in which dialogue is not subordinated to public rules and procedures. It could happen, therefore, that solutions reached in the private sphere violate certain general principles and 
individual rights. At the same time, however, any interference with the private sphere, made in the name of individual rights, could violate the very basis of the existence of lower-order groups. ${ }^{318}$

Finally, in Habermas' model the public sphere reaches its perfect form in the period when participation in it is limited to representatives of a single social group, namely the bourgeoisie. Surely, as Benhabib demonstrates, Habermas' locating of the model of public sphere in the first period of the development of the bourgeois society does not preclude the possibility of extending it by embracing further groups in the rational discourse. However, Habermas himself sees the extension of the right to vote as the beginning of the crisis of this sphere because the broadened circle of citizens came to include groups whose class interests were contradictory and impossible to reconcile. Thus, in the new social reality - similar to the contemporary one - it ceased to be possible to uphold the concept of a rational discussion that would allow reaching consensus.

This is a kind of "aesthetic" defect found in the most laudable models of democracy, namely that they endorse solutions that exclude a number of groups or social issues from public life. Still, this "aesthetic" defect is far less important than the "theoretical" one which arises from the fact that the public sphere cannot be considered in separation from its complementary concept of the private sphere regarded not in terms of individual intimacy but in terms of intragroup spaces. This dilemma was already pointed out by Hegel, who underlined that the law can be only personal, making the individual its subject and consequently a participant in the public sphere. However, if it were indeed the case that individuals themselves create the public sphere, the state would deal with a mass - an inorganic, disordered collectivity. Thus, individuals need to be subordinated to certain circles, groups or communities, among which the family is the most important one, and whose internal space - the "private sphere" in the original, historical sense of the term - does not constitute a subject of the law. Therefore, the condition of the existence of the public sphere would be the existence of citizens being rooted in quasi-private groups whose values and interests they could represent in relation to the state. It is precisely at this point that we encounter the contemporary understanding of the concept of the civic society as an entity that lies at the foundation of a democratic state.

The above understanding of the concept of the public sphere conforms to a particular semantic aspect identifiable through lexicographical analysis, namely

318 As in the case of taking children away from their parents due to poverty, "inefficiency" of care, or on suspicion of violating children's rights. 
one indicating that this sphere constitutes an inter-group space, a sort of a meta-level that acts as a place for the integration of diverse groups, not the individual members of a given society, who were extracted from it. Moreover, one could also easily demonstrate that although individuals are the main actors in the public sphere in models developed by Arendt and Habermas, even in these theories individuals represent greater communities. This is not only because their actions and decisions affect the position of those who are excluded from the public sphere yet remain tied to its full members through family or economic bonds, but also because it is the quality of the private sphere that ought to be regarded as the source of their unique features and competences (which are assumed to exist by Arendt ${ }^{319}$ ) or the character of their particular interests (emphasized by Habermas).

Therefore, the public sphere - as the domain that determines the rules and goals of collective life - needs to be complemented not with a private sphere of individual decisions but with an inter-group space in which individual members of the society are rooted and from which they derive their ideas of a "good life."

\subsection{The concept of the public sphere}

The above survey of meanings related to the concept of the public sphere shows that it is rich in associations and offers many intuitions, among which the foremost are: (1) generalizing and sharing choices and behaviors within (2) organized space, entrance to which is regulated by (3) general criteria or rules of passage, which create (4) a "meta-group" of an abstract (general) identity, which goes beyond the private (intra-group) life-world of individuals. Analyzing the historical transformations of meanings related to the concept of the public sphere can also help demonstrate the existence of two processes which inform a different distribution of emphasis in accounts of phenomena related to it. The first concerns the disappearance of private shared spaces. ${ }^{320}$ Public spaces of the "lower order" (such as house, street, neighborhood, workplace) have begun to

319 In this perspective, the morality of private communities becomes the source of public virtue. Even though at first glance the idea that the best models of living together (developed in the private sphere) permeate into the public sphere seems utopian, it corresponds to the theories of Auguste Comte, Adam Smith and even Charles Darwin, who has expressed (in a passage quoted in Chapter Four, note 16) the conviction that long-term success shall be secured by those human communities that are capable of developing moral virtues among their members to a greater extent than others.

320 In fig. 7.1. they are represented by circles inside the greater whole. 
be the meeting place for individuals who do not share any "local" sense of community or a "local" common good. ${ }^{321}$ The second process, historically parallel, regards the extension of the state's authority over the entire public space, which has been opening up along with the disappearance of "local orders." As a result of this process, individuals have become the only "particularity," while the state the only creator and guardian of the public sphere.

Therefore, the question arises whether - given the richness of associations with the term - there actually exists something that could be regarded either as an element shared by all the diverse ideas and theories developed around it, or at least as a condition necessary for the emergence of the public sphere, regardless of the details specific to its various accounts. If we were to assume that the overriding function of the public sphere is to establish and continuously coordinate actions in larger collectivities comprised of individuals and social groups, then its necessary condition would consists in creating and confirming common knowledge about what other people feel, know, do or plan to do (Chwe 2001). ${ }^{322}$ After all, actions in the public sphere can be coordinated only under the condition that individuals are capable of attributing to others knowledge which they possess themselves, and predict their behavior on this basis. ${ }^{323}$ Thus, in order to grasp how the public sphere functions it is crucial to understand social processes of forming common knowledge.

In the perspective developed here, the public sphere is therefore a space facilitating public disclosure of knowledge, intentions and sentiments, either in the form of behavior (actions) or verbal communication ${ }^{324}$ by making them public and thus constituting common knowledge, co-intentionality and co-feeling. It needs to be emphasized that common knowledge assumes not only that everybody knows

321 It is this aspect of transformations that is pointed out by Richard Sennett (1992) in his discussion of the conditions for the emergence of the "public man" and his later "fall."

322 This definition refers to the theory of common knowledge understood as a condition for coordinating actions. As Suk-Young Chwe writes (2001), knowledge of a certain message does not necessarily facilitate coordinated action - it is also necessary to know that others received the same message, understood it in the same way, and intended to act in accordance with that particular information.

323 Drivers move in a coordinated manner not only because all of them know traffic regulations and signs but also because each of them additionally assumes that others know them and will act in accordance with that knowledge.

324 Just like language, behavior reveals emotions, intentions and knowledge, but does so in non-verbal ways. 
the same thing but also, additionally, that everybody knows that others know this, and that the latter know that all the rest know this. Next, co-intentionality assumes not only having knowledge of other people's intentions regarding a certain action plan, but additionally having the intention that is a part of this plan. Finally, co-feeling assumes not only experiencing the same feelings but also the conviction that others feel the same (Tomasello 1999). ${ }^{325}$

It is in this public community of convictions, sentiments and intentions that one ought to seek the source not only of the regularities that create the concept of the society as a system, but also of the idea of the common good as an overriding normative principle governing the decision-making process in the public sphere. Thus, participation in the public sphere invariably entails a component that ethnomethodologists have termed knowledge taken-forgranted: the conviction that others understand signs in the same way, know the same rules, hear and see the same things, and so forth. ${ }^{326}$ This also entails the conviction that there exists a certain state of reality that is beneficial for all participants in the public sphere - it is only by assuming this view that it becomes possible to expect cooperation from others acting in the public sphere. Finally, such participation presupposes an internal compulsion to act in a certain way due to the consciousness of a common knowledge shared among the people who constitute the auditorium, i.e. the "audience" of individual behavior.

Now, let us draw attention to the fact that, given such a view of the public sphere, it becomes to a certain extent identical with the concepts of community and sociality. One could even say that - in light of the discussion in Chapter Two - the essence of the "foundational holism" (which constitutes the basis of sociality) was the making public of individual thinking, e.g. in the form of cave drawings, or through the possibility of experiencing the same emotions while participating in shamanic rituals. Similarly, Linnda Caporeal, whose theoretical

325 This is perfectly illustrated in the well-known fairy tale by Hans Christian Andersen about the emperor's new clothes: although all people in the crowd who witness the emperor's procession individually do see (and know) that the ruler is naked, none of them are certain whether others see the same thing. It is only the making public of this knowledge by the little boy's cry, in combination with eye contact, allowing to observe other people's reactions, that renders this distributed cognition common and thereby facilitates the coordination of actions.

326 One's own knowledge and knowledge about other people's knowledge (metaknowledge) also constitutes an important element of phenomenological sociology, analyzing so-called "constructs of the first and second order." 
model is presented in Chapter Four, argues that the proper function of deme is the creation of shared knowledge, which constitutes the basic condition for the realization of the fundamental task of the base community: the coordination of individual actions.

These similarities should not come as a surprise because - as we have already demonstrated - every community creates its own, "private" or "local" public sphere, which bears the same characteristics as the public sphere in superior collectivities. At the same time, this similarity facilitates grasping the circumstances of the latter's development - as a space of integration and inter-group coordination, it emerged at that stage of historical development in which, along with the numerical growth of the primal communities, there arose the necessity to construct a political order on top of the social one. This was related to the rise of the state - a concept that, as we have indicated in Chapter One, is frequently confused in sociology with that of society, despite the fact that those two entities function in different dimensions of reality and refer to different mechanisms of generating common knowledge as the basis for coordination of actions. The state employs primarily formal institutions, especially law, whereas processes of socializing human action are founded mainly on the informal institutionalization of behavior, which is thus transformed into habits and traditions.

These differences are the subject of the next chapter - the present discussion focuses on broader characteristics of public sphere, which manifest themselves regardless of whether it assumes a more "local" or more "universal" form. They could be described by indicating three fundamental functions performed when knowledge, sentiments and intentions are made public, both in local communities and higher state collectivities.

Certainly, the most primal and simultaneously fundamental function is the coordination of individual and collective actions in a way that raises the degree of integration in the entire social system. In this respect, it is essential for each member to know his or her place and task within the broader community. Many tools are used to achieve this goal: elaborate ideological systems as well as parables, sayings and proverbs that are part of common knowledge. On the one hand, therefore, these are visions of order, in which each estate has its own ascribed function in the organic social whole; class ideologies that assign to each collective normatively specified tasks; or concepts of individual rights. On the other hand, there are tools such as popular literature, films and proverbs like "children should be seen and not heard" or clichés like "women's vocation is to bear children."

In the area of integration, the most fundamental task performed by the public sphere is not only to turn distributed knowledge into a common one but also to 
legitimatize it so that it can become indubitable and taken-for-granted. ${ }^{327}$ One could also say that integration occurs primarily by way of broadly understood education and the creation of what Pierre Bourdieu has called "habitus," which constitutes a set of dispositions to act within the framework of structures and institutions in the superior system. This goal is served not only by journeyman institutions or ones facilitating general education but also by establishing and celebrating religious and state holidays ${ }^{328}$ - the goal of which would be to develop and sustain a sense of the collective "We" superior to both individual and group identities.

The second vital function of the public sphere is to mobilize the population in order to accomplish goals whose achievement requires the cooperation of a greater number of people, e.g. taking revenge on another tribe or family, proper maintaining of irrigation canals, constructing dams, defending the state against enemy invasions, or - conversely - enlarging it by territorial expansion. Conceived in this way, the function of the public sphere would boil down to generating engagement and participation, which become the basis for cooperation, whereas the fundamental space for its emergence would be a tribal or political assembly, a meeting of allies, or a discussion club. In all three cases, the goal is to generate common knowledge (e.g. what everyone thinks and proposes to do) in order to decide about the joint course of action in the course of a more or less rational debate.

Regardless of differences between theories of the public sphere, all of them view it as a space that is crucial for social life in its political dimension: one where agreement is reached with regard to goals that bind all interested parties. Thus, key aspects in the area of mobilization include not only the release of emotional energy by reinforcing a belief in the common cause but also the selection of the mode in which decisions are made in the process of public discourse and construction of action plans in accordance with a broadly accepted concept of the common good. It is in this last category that the realm of politics is located as an area of decision-making and citizen participation. A vital role in mobilizing society is also played by public rituals whose essence is to "generate" an audience that legitimizes existing structures of coordination, especially those that

327 It is not essential, however, that individuals see it this way - it suffices that they believe that others do not doubt it.

328 An identical role is played by family celebrations, which not only "publicly" renew bonds between people but also confirm the roles they play within the framework of a "private" community. 
embody power relations. The passage of the royal procession not only reminds of, and makes present, the authority of power, but also - by creating an audience - generates common knowledge and sentiments. Every public ritual - be it Gaudeamus, knighting or the opening of the Olympic Games - performs a similar function: it restores a sense of the common good and a community of sentiments, consecrating all actions undertaken in the name of the community.

Finally, the third vital function of the public sphere is to facilitate individual and collective actions. After all, the public sphere is also the space of coexistence, in which the actions of particular individuals are woven together. Their coordination demands not only sharing knowledge but also establishing and obeying rules that would facilitate the accomplishment of personal goals for everyone, or finding satisfaction by acting in that field. A case in point here would be a public road used by riders or drivers, or a bath house and cinema, which attract those desiring to bath or entertain themselves with films. The organization of these spaces is supposed to ensure conflict-free and effective sharing of a certain good (road, bath or film) in such a way that individuals do not endanger others, violate their rights, or diminish their chances of pursuing desired goals, etc.

In this sense, the public sphere secures, facilitates and sustains the coordination of actions, mediating between individuals and determining trajectories of their behavior. Clear traffic regulations and signs, alternating bathing days for men and women, or numbered seats in the cinema are all mechanisms of control, which force individuals to accept public roles. These mechanisms and common knowledge (in the sense specified above as the knowledge about everyone else knowing what one knows) are the condition for sustaining the coordination of actions and, by the same token, the condition that needs to be met if individuals are to achieve their particular (private) goals. The public sphere is constituted through various techniques of generating common knowledge (trainings, courses, tips from other people or learning from them), rules of access or licensing individuals (ticket, library card), and organization of physical space (signs, numbered chairs in public halls, etc.). Its main function is to minimize the probability of conflict, collision or disturbance among individuals using the same space for their own goals.

To guarantee collision-free space, it is necessary to obey rules and procedures, and simultaneously suspend private identities in favor of public roles. Regulations secure a peaceful and harmonious joint movement of individuals, whose individual actions are coordinated even when they are completely independent of each other. ${ }^{329}$ More than that - any attempts at making such actions common

329 In this context, the contemporary driver's knowledge that it is advisable to pull over upon hearing an ambulance siren would correspond to the knowledge of a medieval 
could endanger the audience itself, e.g. in the case of hooligans on stadiums, quarrels between spouses while driving on a motorway, or the conversion of a café into a drug den. In all these cases we deal with the invasion of particular identities, which transform public sphere into a private one. Thus, the rules that facilitate actions in the public sphere primarily aim to block the expression of knowledge and sentiments as well as to cool and formalize contacts. One emotional outburst on a motorway could lead to a pile-up, and one insubordinate audience member is enough to transform a film screening into a brawl.

However, lack of collisions does not exhaust the entire space of coexistence created by the public sphere. It can be also filled with positive emotions as a place "where people who do not know each other can meet and enjoy each other's company" (Ariès 1989, 9). Coexistence in public space can also have a festive and celebratory character, giving a sense of "revealing oneself" to others and presenting one's individuality. A good example of this would be a stroll on a promenade at a holiday resort. As the interactionists emphasize (cf. Goffman 1967; Collins 1975), presence of many individuals in a single space constitutes a point of departure for the development of complex interactionist rituals whose goals include the confirmation of individuals' position with regard to others.

It needs to be added that the development of this specific function of public sphere - i.e. facilitation of individual actions - is a civilizational invention that does not go very far back in the history. As Norbert Elias demonstrates (2000), the use of the public space for individual actions was based, for a long time in history, on direct regulations, or the "law of the stronger," which would apply when particular interests come into conflict. According to Sennett (1992), the impulse that prompted the development of modern public life in London and Paris - the largest Western cities in the middle of the eighteenth century - was the appearance of "aliens:" thousands of young people arriving from remote parts of England and France in search of employment. The myriad of immigrants dispersed in London and Paris, without any social roots, alongside the new middle class which was engaged in an exchange of products rather than their production and which did not have enough time to develop its own professional ethos (both categories partially overlapped). All these people did not yet have a defined social identity. The effect of this was the emergence of specific patterns of social interactions (especially since their intensity was greatly increased) among people

citizen, who understands that it is best for him to find shelter at a wall when a procession on horseback is approaching. 
who would not know each other's status. The culture of public life was based at that time on the assumption that, in the public sphere, one plays a certain role rather than presents his or her identity. ${ }^{330}$

The above description of basic functions also allows to indicate those shared features of public space that are necessary for the making public and common of sentiments, knowledge and intentions.

First, public space is by definition accessible so as to make it possible for new participants to join in out of their own will - not on the basis of drafting or nominating. Let us note that the public character of a given space does not require the kind of accessibility that would be completely unhindered. After all, for example, only registered members can use public libraries. However, the public character of these institutions is determined by the type of limitation - anyone who fulfils certain criteria can register as a library member. Other public spaces also utilize certain modes of licensing, qualifying, ticketing or restricting access (e.g. one cannot walk naked in the street). The idea of universal, free and unrestricted access to the public sphere is not even realized in the space of home to the extent that other household members always have to be taken into account. One could adopt a similar perspective with regard to the "exclusive" character of the Greek polis or the bourgeois public sphere, indicating that the evolution of the conditions of access to the public sphere was one of the vital factors in determining the historical transformation of the political variant of the public sphere.

Second, public space is always a space of making something apparent to others: revealing oneself or something (one's competences, reasons, opinions etc.), even when this simply means "being exposed to the public view" as in the case of enjoying a walk or travelling by tram. In the original sense, making public means communicating to the public the ideas, reasons, information or arguments formulated or created by various groups or individuals so that they can be known to other members and become the subject of discussions. As

330 As Sennett emphasizes, public life, which blossomed in the mid-eighteenth century in the capitals of Britain and France, was based on the universally shared assumption that a certain distance exists between personal identity and the role one plays in public situations. This conviction allowed people to play with one's identity, as part of which they would create themselves. According to Sennett, the equating of one's self-image presented in the public with one's true nature was the main symptom of the crisis of public life. Its first signs, Sennett argues, can be observed already in the mid-nineteenth century. The equating of the role with identity subjected individuals to social control (cf. Foucault 1979), as people assumed that, based on observation of a person in the public, one was able to judge who that person really was. 
Linnda Caporael indicates (1995), making things public always provides grounds for spontaneous group action. Cries signaling that a dam or a tram is broken can cause the actions of passers-by or fellow travelers to acquire a common direction. Transformations of the strictly technical means of making things public, which has occurred along with the development of mass communication, are therefore an important factor determining the historical changes of the public sphere.

Third, public space is always somewhat organized, not only in the sense that there are rules and signs aiding a larger number of participants to coexist inside it and identify the more or less clearly defined boundary between the public and the private, between what can be revealed to the broader public and what ought to remain a "bedroom secret." It is also organized by virtue of the fact that formal institutions operate inside it, whose main task is precisely to make knowledge common and public, and take decisions in the name of the entire collectivity, later supervising their implementation. ${ }^{331}$ The basic institution of the public sphere is naturally the state, along with all of its branches. However, institutions of this kind can be also created on the grassroots level out of the initiative of particular individuals or social groups, or on the basis of their agreement. The crucial property of public space - one that organizes its functioning - is the (often situationally constructed) division into leading actors and audience.

The theory of public sphere developed here proposes to treat it as a space where actions are coordinated by way of making sentiments, knowledge and intentions public and common. This allows one to discuss its historical transformations somewhat differently than the classical theoretical models presented above. If the public sphere is regarded as constituted by a particularly understood space, a "meta-group" recruited in some way, and specific technologies of making public, it would become easier to point out those of its changes that are related to the emergence of mass media as a new technology, mass society as a new type of "meta-group," and cities as well as other modern forums on which people meet as new forms of public space.

Doubtless, the transformations of public spaces, media and "meta-groups" bear consequences for both the way in which the three main functions of the public sphere are realized, and for the effectiveness of its deployment in establishing or sustaining the coordination of social actions. Thus, since the frequently underlined demise of the public sphere is described by indicating symptoms of

331 The counterpart of such formal institutions in "local" communities would be - for example - the division of roles in the family or the informal authority of the "council of elders" or most distinguished family members. 
crisis in the domain of the coordination of actions - both in terms of social integration (sense of similarity and sharing one's fate with others), mobilization (participation), and facilitation (sense of control over one's actions) - this does not have to mean that we are facing a real crisis of the public sphere. All it might prove is that currently operating institutions, rules of behavior and actors have lost their power of generating common knowledge, intentions and sentiments in favor of other spheres - developed in the former's shadow - where coordination is established and sustained.

Let us therefore examine in detail the transformations of the public sphere in human history.

\subsection{From tribal to political community - traditional societies}

Although the earliest tribal communities did not make any distinction between the social and political orders, this does not mean that we cannot speak in their case of the existence of such a space for action that would correspond to the public sphere understood in the way proposed here. As we have already noted, generating common knowledge is the basic function of deme, comprised in this particular case by the entire tribal community. These communities also had to take decisions with regard to all their members (e.g. about declaring war on neighboring tribes) and create adequate means of mobilization and control, which would prevent the problems of "social idleness" or "free riding," invariably emerging at all sites of collective action.

As scholars researching such communities unanimously emphasize, probably the clearest feature of the public spheres they produced is their total inclusiveness. Decisions were taken in them with the participation of all adult members of the community gathered at a large assembly; moreover, they would be taken unanimously. Claude Lévi-Strauss shows that some communities also developed specific rituals whose task would be - to use the terminology proposed in this study - to facilitate actions of this type. "When an important decision has to be taken, a kind of ritual combat is organized a day or two days before so that all old quarrels are liquidated in fights;" community, he concludes, begins from cleansing itself of all grounds for disagreement, "and it is only when this has been done that the refreshed and rejuvenated community, having rid itself of all differences of opinion, is in a position to take a decision which will be unanimous and thus express common good will" (Charbonnier 1969, 35).

Regardless of their subject matter, these decisions are subordinated to the general image of the world, which coordinates both the process of making such choices and the everyday activities of such communities. Despite local variations, 
this image is - in its basic forms - always the same. It is saturated with the presence of the sacred and based on mechanisms to which keys are held by gods, demons and secret powers. Thus, if the well-being of both the entire community and its individual members relies primarily on such powers, influencing them is possible only through religion ${ }^{332}$ or, more precisely, through religious rituals aimed to win the favor of gods and gain mastery over the secret powers that rule both nature and the fate of individuals.

While analyzing the role of religion in the integration of the collectivity, Peter L. Berger draws attention to the fact that the legitimization of a given vision of reality cannot be limited to sustaining belief in it as part of collective actions, even when this is supported by disciplining measures. As he succinctly puts it, "[m]en forget. They must, therefore, be reminded over and over again" (1967, 40). Ritual is the key means of "refreshing their memory." Its power rests chiefly in that "[a]gain and again it 'makes present' to those who participate in it the fundamental reality-definitions and their appropriate legitimations" (40). Ritual restores "the continuity between the present moment and the societal tradition, placing the experiences of the individual and the various groups of the society in the context of a history (fictitious or not) that transcends them all" (40-41). At the same time, it gives the feeling - by reassuring or convincing - that others share the same reality as we; in other words, ritual helps not only to refresh common knowledge but also to confirm that it is common.

Thus, ritual does not only aid memory. Its fundamental and oldest function is - as we demonstrate in Chapter Two - to establish emotional bonds between community members, or - strictly speaking - to create that community. ${ }^{333}$ This was already emphasized by Durkheim, who wrote that "the cult really does periodically recreate a moral entity on which we depend, as it depends on us. And this entity does exist: it is society" (2001, 258; emphasis added). According to Durkheim, ritual has two crucial features that enable it to realize this function. First, it is performed in the presence of a larger group gathered in one place. Referring to the terminology used here, one could say that rituals constitute a

332 Durkheim draws attention to this when he writes that "at least the manifest function of the cult is initially to regularize the course of natural phenomena" $(2001,259)$.

333 It is symptomatic that Berger completely disregards this function of rituals. Both The Sacred Canopy and the book co-written with Thomas Luckmann contain no references to social bonds. This becomes clearer if we recall that the interpretative paradigm, to which both works have contributed significantly, focuses on individuals as the main social actors, and on cognitive processes as the basic mechanisms guiding their actions. 
way of making public the vision of the world, at the same time allowing people to observe each other as well as each other's reactions and reactions to these reactions, etc. "Their first effect, then, is to bring individuals together, to increase contacts between them, and to make those contacts more intimate. This in itself causes a change of consciousness" (258). ${ }^{334}$

Second, ritual transports us from the realms of the profane into that of the sacred not only because it is related to beliefs in the narrow sense but also because it somewhat "suspends" everyday reality along with all of its down-toearth existential problems. Solving such problems is subordinated to the self-interest of individuals, setting them in an irresolvable conflict with other members of the community. As a result of such suspension, thoughts are focused on social things: "[e]ven the material interests that the great religious ceremonies are meant to satisfy are public in nature, and hence social. The whole society is interested in the abundance of the harvest, in timely but not excessive rainfall, in the regular multiplication of the animals" (259). ${ }^{335}$ Due to the public character of

334 Changes in the contents of consciousness caused by the physical closeness of people gathered at the same place were indicated by Durkheim's contemporary - Gustave Le Bon. In The Crowd. A Study of the Popular Mind, he writes: "The sentiments and ideas of all the persons in the gathering take one and the same direction, and their conscious personality vanishes. A collective mind is formed, doubtless transitory, but presenting very clearly defined characteristics" $(2001,1-2)$. Unfortunately, due to the very suggestiveness of the claims made in this work, it has shaped the image of the crowd as an entity that is - by its very nature - irrational and subject to uncontrollable emotions. It also affected research on phenomena occurring in larger gatherings of people, subordinating them to an analysis of the internal dynamic of collective behavior and the means of manipulating it.

335 In contemporary sociology, one of the few concepts in which the coexistence of individuals in a single space would be regarded as an initial condition for the development of elaborate rituals of interaction is the theory of Randall Collins. He begins with a Durkheimian analysis of religious ritual but assumes that "[t]he bonds that unite human beings are fundamentally the same as those that unite other animals. The only difference is that humans have symbols that can invoke unseen realities and hence carry the past into the future. Religious and other moral ceremonies thus serve to attach the animal bonds of emotion to symbols which enable people to carry their solidarity in their heads even when they are not together physically" $(1975,95)$. Thus, just like Berger, he sees ritual primarily as a kind of "mnemotechnic" tool: it would help one remind him- or herself that something links them to other members of the community. By the same token, this entails a complete disappearance of the specific function of ritual which, according to Durkheim, is to create the concept of the common good. 
rituals, they allow not only generating common knowledge but also intentions and sentiments, thus renewing or recreating the very basis for coordinated social action.

Religious rituals are therefore a means of sustaining the idea of the common good, which grows out of the totality of social praxis, and is set within an image of reality that is specific to a given community. This idea constitutes the basis for integrating a system of coordinated actions. Due to its relations with the basic factors determining lifestyles, the common good is something that every member of the community wishes to come to existence, regardless of any existing divisions. Let us note that the common good, if understood in this way, is not identical with a "community of values" and cannot be reduced to accepting a "normative system" common to all community members. It is something concrete, tangible, and definable in terms of clear, specific states of reality - i.e. as abundant crops, turning away of plague, courage in facing enemy - ultimately resulting in the rise of well-being as well as an increase in the community's significance and, consequently, in the significance of all its individual members.

The rise of chiefdoms and states as "management" or administrative units caused by further population growth, certain demographic processes, or conquests - unleashed processes that invariably began to fragment the initial "totality" of the original community, which concentrated in itself all dimensions of individual existences. It was a split into rivalling clans and separate factions not just individuals but groups of warriors, clerks, specialized craftsmen - developing a praxis that differed from what was experienced by other members of the tribe. The right to make decisions was centralized, just like the right to use violence. Access to certain information was limited only to the chieftain and his closest associates.

Still, it is the religious system that integrates the community into a superior unit. On the one hand, it incorporates all emerging divisions into a coherent image of the world, in which they become functionally justified. On the other, the very existence of these divisions is thus legitimized. The religious system is also a means of sustaining social identity, which - along with the shared language - is based on some kind of a cult of ancestors, which secures the continuity of the collective "We" on various levels of the superior collectivity, and helps control the forces of nature. The fundamental way of making common knowledge public is still the ritual, in which the figure of the ruler, who acquired divine status, begin to play an increasingly important role.

The transformation of certain chiefdoms into states, which occurred along with conquests as well as consolidation, only seemingly changed this state of affairs. Despite certain similarities to modern states (centralized power, often 
based on bureaucracy, specialized administration, separation of trade, existence of cities, etc.), ancient empires were basically a more or less loose confederation of communities based on clans or extended families, which served as the basic point of reference for identification and integration of most people. The organization of cities was also based on a family system. As Max Weber shows in his compelling analysis of the phenomenon of urbanity, ancient cities were primarily fortresses as well as administrative and cult centers established with the help of local people, most often coerced by force of arms. Nonetheless, the group of forced settlers "retain[ed] the membership in its former local and clan associations" (1978, 1245).

One could therefore say that, despite further differentiation of ancient societies, ${ }^{336}$ it is still easy to point out what they believe to be the common good. After all, the well-being of all - godlike rulers and their clerks, warriors and priests, as well as all other folk - depends primarily on the still unconquered forces of nature. Influencing them is possible only by way of religion. As Hans Georg Wunderlich suggestively demonstrates, using the example of Egypt, this was the main aim of elaborate cults of ancestors, which produced monumental constructions admired to this day. In Ancient Egypt, the dead pharaoh was typically regarded as an earthly incarnation of Osiris, the god of vegetation and of the dead. Therefore, it was requisite to erect a funeral monument that would correspond to the creative powers of the whole people. Failure to do so, Wunderlich observes, "would bring down dire punishment upon the whole country. Above all, it might mean a failure of the Nile flood and hence the end of vegetation, which was directly dependent on Osiris and his earthly representative. Of course, the masses of people [constructing the monument] had to be guided, but force was not involved. The inner coercion of religion was effective enough" $(1974,333)$.

Naturally, identifying and acknowledging common good does not preclude conflicts between rulers and their subjects. The existence of such conflicts in ancient states is confirmed by numerous mentions of rebellions against excessive rents imposed by particular rulers. However, questioning of specific decisions

336 One needs to remember, however, that this process of differentiation had a relatively narrow reach. Jacek Kochanowicz reminds us of this: "Despite the impressive development of cities, religion, art and intellectual life, the ordinary masses in these societies - some 80 per cent of all people - were constituted by illiterate peasants living on the verge of starvation and working in a pre-industrial economy aimed at survival. Upper classes lived off wars and taxes, basically exploiting peasants, i.e. supporting themselves with redistribution" $(2003,467)$. 
does not entail undermining the ruler's right to make them, his special role in upholding divine order, or that divine order itself. Even if it is violated by actions of particular individuals, this does not mean that the entire community fails to understand what proper behavior is. Were someone to forget about it, early legal system provided for strict punishment, which would immediately refresh that person's memory.

Let us note that, along with the transformation of tribal communities and chiefdoms into state organisms, the mode in which common knowledge was made public also changed. Strictly speaking, it was enriched with new techniques, unknown in earlier forms of social organization. This was related primarily to the invention of writing. Initially, as Lévi-Strauss shows, it was used "for inventories, catalogues, censuses, laws and instructions; in all instances, whether the aim was to keep a check on material possessions of human beings, it is evidence of the power exercised by some men" (Charbonnier 1969, 30). Although knowledge of writing was not widespread, in Sumer - where the earliest written documents were found - it was known not only by priests and professional writers but also, at least to some extent, by tradesmen and administration officials.

Writing greatly accelerates the popularization of common knowledge about ways of acting established within the community. This is because it does not require personal interaction when transmitting information. Additionally, it diminishes the risk of distortions, which may occur in verbal communication. Naturally, in societies using solely oral methods of passing down tradition, special techniques of memorizing even very long texts were developed, but this was limited primarily to texts of fundamental importance for the collective identity of a given community, ones documenting its history and customs (laws). Moreover, the making public of a given canonical text would always demand the physical presence of a person specialized in memorizing it. ${ }^{337}$ The invention of writing makes it possible to overcome such limitations.

First of all, writing facilitated faster transmission of information to the broader public. It also made it easier to integrate various communities, dispersed in a larger area, into a single political and economic organism, especially when accompanied by an efficient system of territorial communication. Already in the first millennium BC, the Assyrian Empire could boast an elaborate system of

337 As Karol Modzelewski demonstrates (2004), before Salic law was codified in writing, it was stored for many generations in memories of select members of the local community, who also performed the function of judges. The same was true for other legal systems used by peoples of the "barbaric Europe." 
relays of horses and postal roads, which allowed governors of individual provinces to maintain close everyday contact with central authorities (Saggs 1962, 234). Diplomatic correspondence also rapidly grew in volume, covering not only relations between rulers of neighboring states but also the protection of life and interests of one state's "citizens" living on the territory of another. On the basis of preserved letters one can suppose that authorities made efforts to lend the public space such character that would help it facilitate the development of trade (protection of caravans, maintenance of crossings, development of water communication, etc.).

Law played a very important role in these efforts. In the Code of Hammurabi, ${ }^{338}$ we can naturally find regulations regarding actions taken in the private sphere (adultery, mariticide, beating of father by son). However, just like the Salic law, these provisions not only regard relations between members of an increasingly diversified social world (i.e. free people and slaves, owners and leaseholders, partners in trade ventures, men and women, parents and children), but also order actions in the public sphere (i.e. with regard to corrupt judges; severe punishment for stealing temple property; unlawful felling of trees; lease regulations; construction and renovation of houses; regulations introducing something reminiscent of "group responsibility" for doctors and construction workers, etc.) (cf. Saggs 1962, 196-228). One could therefore say that these codices constituted a mechanism of adapting rules of behavior to the changing reality, which began to be comprised not only of the space regulated by rules of coexistence within communities (included into codices) but also public space, which is, in a sense, "no one's." In this way, by imposing a common framework of actions in the intergroup space, they would become means of political integration.

Up to a certain moment, the world of Greeks and Romans was a continuation of earlier historical societies. The myth of the golden age, presented in one of the oldest Greek works of literature, the long poem by Hesiod, contains traces of elements characteristic for tribal communities (Graves 1992, 36). However, in Hesiod's Greece humans found themselves already excluded from the golden age; moreover, they had already moved past the epoch of kings, and existed - as Pierre Vidal-Naquet shows - "only by means of agricultural labor carried out in

338 The Code of Hammurabi is younger by entire three centuries than the oldest known example of written law, which also comes from Babylon, specifically from the period of the reign of King Ur-Nammu of Ur (2111-2094 BC). In later findings, we encounter the same problems that ancient lawmakers repeatedly attempted to solve. 
the heart of the familial community, the oikos" $(1986,2){ }^{339}$ This is part of more general historical changes that transformed Greece in the period after the fall of Mycenae and the great migration. ${ }^{340}$ The subsequent period of cultural and political revival greatly extended the scope of this "family." "In the fourth century, the concept of Hellenism continues to be cultural: a Greek is someone who has received a Greek education, which someone born a barbarian is capable of acquiring" (Vidal-Naquet 1986, 4); in other words - a Greek is someone who behaves like a Greek. Some two centuries later, however, the difference between Greeks and barbarians began to be emphasized in stronger terms by referring to the opposition "between the slaves of law and the slaves of a despot" (4). ${ }^{341}$ Crucially, however, between the world of the polis (denoting the collective "We") and the world of barbarians (denoting the "aliens") there emerges ethnos - a Greek community in terms of dialect and culture yet one that could not organize itself into a city-state and became subject to the rule of tyrants (Żelazny 2004, 25).

The rule of law is, therefore, the special invention made in Greece which was later raised in Rome to the rank of an organizational model that is still in force today and regulates the relationships among citizens. The inter-group space of

339 The meaning of the original Greek term - as Maria Rogaczewska helped us establish - is much richer than what we associate with the concept of family today since it indicates a place where one returns, and one's natural environment, where he or she can flourish. It is also related to the concept of the "ecumene," which denotes a community both in territorial and cultural terms (today - in the spiritual sense).

340 Wunderlich (1974) developed a very interesting theory, according to which the roots of the modern Western European civilization ought to be sought in the specific transformations of the cult of ancestors in Greece, which morphed into the cult of heroes. In this view, heroes (who were born out of gods and mortals) became models for their worshippers, who could make attempts at imitating them (after all, gods cannot be mimicked). The basic edifices in this "cult" would be theatre, where scenes from the lives of dead heroes were staged, and the stadium, where bodies were exercised so that they could achieve feats matching those of heroes. The twilight of the cult of ancestors and religiosity as such (only as late as in the Hellenic period of Greek history) is indicated also by Anna Świderkówna (1978, Chapter Ten). Cf. also Pierre Vidal-Naquet's discussion of the changes in how the Greeks conceived time (1986, 39-52).

341 This change was inseparably linked with the development of Greek philosophy, which preceded it and flourished in the period between the fifth and fourth century BC. However, it is important to stress that its broader reception, even in a society where all citizens could read and write, took around two centuries. 
integration was subjected not only to customary but also to formal regulation created in a bottom-up way through the collective will of all citizens. ${ }^{342}$ Along with the rule of law another process began: the centralization of executive and judiciary power. The power that was formerly dispersed among local communities crystallized in the form of political institutions. This meant the birth of the public sphere in the form we recognize today, i.e. as a specialized sphere, separated from the social order and programmatically independent from it. It is also in Greece, and then in Rome, that we can trace the first "test of strength" between the private sphere (of great families) and the public sphere. As we have indicated in the chapter on the transformations of family, both in Athens during the classical period and in Rome during the Republic legal reforms were introduced in the area of marriage, inheritance, and citizenship, which put those families at work for the state's political structure: on the one hand, the status of citizen gave fathers new prerogatives inside the family, but on the other made them guardians of the public order.

In other words, heads of families were "extracted" from the communities and given new privileges; their power was augmented insofar as they maintained loyalty to the newly emerged group of politicians managing public matters. Thereby, however, the level of the community lost the rights regarding its internal public sphere, and group responsibility was replaced with individual one. This means that societal self-control was severely limited. Conditions for gaining citizenship, granting rights and holding strictly public roles, as well as awarding privileges or material benefits, became means of strengthening central power.

However, the rule of law concerns mainly the political order. Although the application of law - which evolved, in Greece and particularly in Rome, from customary specific norms into an elaborate abstract system ${ }^{343}$ - certainly affected the perception of reality (especially in circles of people with better education ${ }^{344}$ ),

342 However, it is important to remember that there were restrictions regarding the status of citizens. The right to vote was denied to women, children, slaves, and foreigners.

343 As Marek Zirk-Sadowski indicated to us, besides moral duty Roman law distinguished legal duty, while besides physical persons - legal ones. Further, the introduction of an abstract concept of property facilitated discerning the role played by concepts in developing the image of the world. Apart from Greek philosophy, law was thus the area, in which the transition from the language of action to the language of reflection first began (cf. Chapter Three).

344 As Joseph Vogt points out, the process of Romanization of the provinces conquered by Rome occurred not only by way of granting local notables the status of Roman citizens, but primarily through the promulgation of Roman legal concepts and practice. It was 
most people would still consider the essence of that reality primarily in religious terms. The social order of both these state organisms was firmly reliant on the work of slaves or half-slaves, while the extended family remained the fundamental organizational unit. ${ }^{345}$ As Weber emphasizes, "[Greek] [d]emocracy was initially unable to abolish the organization of the citizenry into sibs (gentes) and the superordinated phratries and phylae [...] [i.e.] purely personal cult associations" $(1978,1245)$. Also within the economic order, the basic sources of income for the population would still be located in agriculture and trade.

One could therefore say that, both in Greece and the Roman Empire, the system of cohering and integrating the state's community was based on two major pillars supporting the organization of life both in the inter- and intragroup space. The first was law, which is no longer rooted only in tradition but also in a specific conceptual system ordering the image of the world. The second was institutionalized religion, with origins in a more or less syncretic system of beliefs. Despite that each of the two - law and religion - concerned a different sphere of life (the profane and the sacred, respectively), they were complementary and supported each other, developing and simultaneously legitimizing the image of the world and the concept of the common good related to it. The public sphere would be divided into two relatively independent yet complementary institutional systems - political and religious - which were made to fit through community's social organization. It was in the extended family, phratry, or phyle that religion and politics would be rejoined, both in the personal sense (with the head of the family representing both orders in his community) and in the behavioral one (in joint rituals).

Thus, given all the important differences introduced by the rule of law, life in Greek and Roman societies was still a continuation of the older, tribal organization of community. ${ }^{346}$ The first departure from this thousands-year-old system of creating and sustaining the moral universe of the community came with the rise of Christianity. In contrast to the religions that preceded it - whose basic function was to collectively influence the forces of nature, on which the fate

law that legitimized the power of Caesars and made service to the state the primary obligation of all citizens (Cf. Vogt 1967).

345 It did not necessarily have to mean real kinship. Weber draws attention to this when he writes that "an association with claims to legitimacy could rest only on the basis of the traditional, ritually oriented organizational forms [...] or at least had to create such a basis by fiction" (1978, 1246; emphasis added).

346 Greek democracy constitutes a crucial modification of the tribal assembly, but it is still only a modification. 
of the entire community depended - Christianity sets entirely different tasks before its believers - tasks assigned to individuals and not to social groups. One could put it even more strongly by saying that the common good was replaced in Christianity with "the good of the individual" because, unlike good crops, salvation cannot be secured with collective efforts of a family- or territory-based community.

What is more, Christianity has "extracted" individuals from their former family relations. "And everyone who has left houses or brothers or sisters or father or mother or wife or children or lands, for My name's sake, shall receive a hundredfold, and inherit eternal life" (Matthew 19:29). They were offered instead to participate in a new community - one no longer based on an ethnic group, but on a more universal system of beliefs ${ }^{347}$ accompanied by an entirely redefined realm of praxis. The aversion and suspicion that the early Christian communes met with in the Roman Empire were caused not only by the difference of their beliefs but also by the difference of their practices. After all, the Roman religious system was eclectic enough to incorporate elements from all kinds of religions practiced at the time (which it actually did). Practices specific to Christianity, however, not only prevented believers from revering Caesars as gods but also led them to question various dimensions of social differentiation, fundamental for the image of the world accepted in the period. One needs to remember that the first Christian communes gave equal rights to all their members regardless of whether they were rich or poor, free or enslaved, barbaric or "civilized," men or women. ${ }^{348}$

In the early stages, Christianity closely resembled an ethos-based group in the sense given to this term by Tadeusz Szawiel (1982), which combines the existence of a well-articulated normative system with the subordination of everyday practices to it. "The distinguishing feature of an ethos is the unity of beliefs and social action" (171). This is exactly this unity that differentiates ethos from other types of normative systems, such as those contained in the traditional

347 However, Zbigniew Mikołejko has drawn our attention to the fact that in Christianity's early years it was not certain whether this religion would not be permanently related to an ethnic community. During the Council of Jerusalem there was a dispute between those willing to keep Christianity within Judaism (James the Less), preserving its laws and traditions (e.g. circumcision), and proponents of a more universal vision (Saint Paul). The leader of the Church, Saint Peter, initially leaned towards the former position and only later embraced one closer to the latter.

348 Just like men, women were entitled to perform a strictly religious function in them, serving as deaconesses. 
understanding of culture. It constitutes one of the main values for members of an ethos-based group, becoming the source of positive self-assessment of a given group on the background of the broader collective, or - as we would put it today - the source of its social identity. Although in the course of later historical transformations Christianity lost that characteristic, it would never abandon attempts at subordinating the sphere of praxis to religiously defined norms of behavior, which found fullest expression in Protestantism, born in the sixteenth century.

There were two convergent processes that determined the evolution of Christianity in the later periods of its development. First, it began to institutionalize itself, developing a separate mode of social organization, one parallel to the Roman state. After edicts issued by Constantine, it acquired the status of state religion. ${ }^{349}$ Such "cohabitation" of the state and church structures is a novelty in the history of societies. This is indicated by Marcel Simon, who writes that "pagan priests formed a single unity with the state, and were basically officials managing religious life. Their privileges did not disturb the unity of the state. Christian clergy, on the other hand, formed a separate hierarchic organization, providing support to a community distinct from that of the state" $(1992,226)$. The struggle between the papacy and the empire during the Middle Ages, and the incessant clashes between "the good of the Church community" and "the good of the state community" became a significant factor shaping the degree of integration in societies which developed at that time.

Second, while extending its institutional influence, the Church was forced to adapt to the reality of praxis, which, in fact, changed dramatically after the fall of the Roman Empire. Its Western territories were occupied by peoples who were still stuck at the stage of tribal organization, with families or clans constituting the basic social unit and exerting full control over the lives of their members, as well as taking group responsibility for their actions. This neighborly-territorial community "would cope quite well with everyday economic problems and

349 Along with the rise of the number of believers and the intensification of centrifugal tendencies that tore the Empire apart in the first centuries CE, the Church became the only organization that had its own centrally bound structures in all provinces. Competing rulers began to regard this as a means of integrating the crumbling state organism. The tolerance edict issued by Emperor Galerius in $311 \mathrm{CE}$, which abolished the persecution of Christians, argues openly that in exchange for the Emperor's generosity, "Christians ought to plead with God that auspiciousness be granted to Us, the state and themselves, so that the state itself could flourish in all aspects as they lead their peaceful lives at the hearth and home" (Simon 1992, 214; emphasis added). 
the protection of traditional order, bringing crime under control and settling disputes, but could not manage more serious military threats on its own. Waging war, negotiating peace and forming alliances belonged to the tasks of the higherorder political community, i.e. the tribe itself, usually comprised of a dozen or several dozen local communities" (Modzelewski 2004, 348).

However, this kind of a higher-order political community had a specific, virtual character. It would not constitute a permanent, independent structure functioning before or after the tribal assembly. Nor did it have at its disposal any executive, administrative or controlling apparatus necessary to enforce the decisions made at the assembly. Therefore, while local communities would be incapable of confronting outside threats without coordinating their actions at a higher political level, the tribe "would not function without neighborly relations because it lacked administrative instruments for protecting peace, mobilizing levy in mass, collecting means for paying political tributes, or organizing construction works to fortify towns and make clearings" (349).

Lack of independent, specialized structures of power and administration leads - as Jan Baszkiewicz argues (1999) - to the dispersion of control, power and violence among communities that keep each other in check. In combination with the principle of group responsibility, typical for barbaric Europe transferring power to lower-order groups was a perfect tool for maintaining relative inter-group balance. A mutual control system effectively blocked any designs on excessive territorial expansion onto shared wastelands, or attempts at increasing political influence.

The basic institution of the public sphere is the assembly, which can occur on three different levels of tribal organization: local, tribal, and supra-tribal. During assemblies of the higher order, the elderly presented the point of view negotiated earlier within a smaller circle. The assembly - "the basic institution of the community, regardless whether formed by neighbors coming together or representing a federation of tribes, always functioned on the same rules" (Modzelewski 2004, 357) - is the most primal form of the public sphere. ${ }^{350}$ On the one hand, disputes are settled during the assembly, which refreshes the "common knowledge" about common law; on the other, it serves as a place for

350 It is worth mentioning here that the specific infrastructure of this form of public space - the shape of the square, ritual attire, and ceremonial character - allowed it to perform the role of generating and sustaining common knowledge. When individuals are shaking spears to indicate acceptance for a particular decision, they can see others doing the same thing; further, they know that others see them, and so on. 
taking decisions regarding matters pertaining to all neighboring communities (or tribal ones at assemblies of the higher order). Moreover, it always has a sacred dimension, which manifests itself in the choice of its site, its principles of peace and non-violence, and the crucial role played by priests, who were responsible for divinatory practices whose results would often affect the decision-making process. Religious ritual constitutes an integral part of the process in which agreement is reached and judgments passed; "the tribe was a community which conceived of its bonds in terms of the sacred" (Modzelewski 2004, 402).

The assembly was not a place where voting would occur: it was a place where unanimity was expressed. Although it seems easy to indicate pragmatic reasons for the emphasis on unanimity, e.g. the fact that - since the local community had to enforce on itself the fulfillment of obligations undertaken during the assembly and everyone had to accept it ${ }^{351}$ - this principle also had a sacred dimension. It was not only the people who had to be unanimous but also gods - any disparity, even in the case of a prophecy, was a bad omen. The principle of unanimity also constitutes a logical complement to the principle of group responsibility.

These basic principles of socio-political organization were retained in the first period after the fall of the Roman Empire, which was accompanied by the destruction of all those structures that bound its population into superior wholes. As Karol Modzelewski has demonstrated in his compelling analysis of the political system of "barbaric Europe," in the period between the fifth and the tenth centuries the dominant organizational form in Europe were still the territorial and neighborly communities. "They satisfied the basic demands of everyday life and work; simultaneously, by protecting order, administering justice, and performing defensive functions they created the basic conditions for the functioning of larger political communities: first tribes and later states" $(2004,343)$. These units, which still functioned in accordance with quasi-tribal, collectivist principles, were provided with the institutions of the new supra-local political power, which developed in constant battles, and of the Christian Church. This occurred not only in the newly Christianized areas but also in Merovingian and Carolingian Gaul, whose structures were developed throughout the six centuries of the Roman rule, and at whose local center of life we find the urban commune (civitas). "[Bishop] Gregory of Tours feels like a citizen of Tour, speaks about

351 Moreover, in the case of passing judgment on someone, objection could set in motion a spiral of violence: if someone did not accept the sentence, then he or she could consider the burning of the guilty party's house as an unjustified act demanding revenge. 
fellow citizens and contrasts them with the citizens of Berry, Mans, Anjou, Bloi, and so on" (Lelong 1967, 14).

The quasi-tribal character of "barbaric" Europe also manifests itself in the fact that the kin-based community was still the basic kind. Based on blood relationships, both in the male and female line (cognatic community), it would place each individual in a complex and slightly different web of dependencies. No individual would exist outside kinship community, just like they could not exist outside a territorial one. The sense of such punishments like political exile or excommunication would be closely related to this particular feature of early medieval society.

However, this world of neighborly and family communities soon began to transform, both under the influence of Christianity and due to processes modifying the very sphere of praxis. As Karol Modzelewski emphasizes, "the Christianization of the barbaric peoples was something much more than an intervention in the sphere of faith and cultic practice. [...] Baptism had the weight of a social revolution: it struck at the very basis of the traditional order in European tribes, shattering it and paving the way for a new order" $(2004,454)$. Just like in the case of the Roman Empire, Christianity "extracted" individuals step by step from traditional relationships, shaping a collective "We," whose distinguishing feature is the community of faith - not the community of origin. ${ }^{352}$ Paradoxically, however, shattering of traditional relationships helped other secular communities to emerge, which would fragment the original, essentially tribal "totality" into distinct worlds.

"The felling of Irminsul, and the dethroning of Svetovid or Perun was [...] a turning point because it caused the cultic unifying force to dissolve in the political organization of tribes. Deprived of their support in the pagan sacred dimension and the authority of prophecy, the lesser and greater tribal assemblies irreversibly lost their significance. At the same time, the king (or prince) gained prominence. Freed from any limitations imposed by the assembly and supported by the Church from the very start, the monarch would utilize traditional prerogatives of the grand relative, mighty warrior and great neighbor in order to transform himself from a tribal leader into a ruler governing a supra-tribal state. The place

352 This community of faith nevertheless draws heavily on metaphors of the community of origin (e.g. "God's children") and family symbolism. Figures of mother, father, son, and the patron saint use the firmly established sense of family community, at the same time reinforcing it. "The devout population came to resemble a vast household divided among numerous residences, each under a protection of a saint or the Virgin" (Duby 1988, 26). 
of the traditional elderly council would be taken in this state by a group assembled around the ruler" (460). Both religion and the taking of decisions about common good were thus moved to a higher level that would be autonomous from society: on the one hand, to the state, and on the other - to the Church as an institution representing the universal community of the faithful. In both cases, new groups of "intermediaries" would emerge, mediating between the level of community and the level of state or religious institutions: lay and church notables as well as officials whose competences included none else than creating and managing the public sphere - parish, church, or a given area of jurisdiction.

However, the alliance formed between the emerging royal power and the Church, which was lifting itself from institutional chaos, did not last too long. Soon, both institutions began to compete for the kind of dominance that would allow subordinating the whole feudal society, which was a patchwork of various local communities of different tribal roots as well as of different languages and traditions, making communication between them very limited. In no way did it resemble the peoples of the "barbaric" period when various tribes would periodically unite in order to boost their chances for survival or expansion. The boundaries between the rising states remained fluid, while the populations of border villages would change hands. From the perspective of an individual, the local (neighborly-territorial) community was the only basic social category, aside from the family, in relation to which the concept of the common good could be meaningfully applied. One could even say that this community constituted a barrier protecting people from outside powers and "law," which attempted to regulate the life of the community from a distance.

The claims of royal (princely) authorities as well as its mightiness are best illustrated by the symbolism developed in that period. Writing about the ceremonies that accompanied the arrival of a king at a city in the Middle Ages, Georges Duby points out that one particularly significant element was the handing over of keys to the city gates: "After all, the key strapped to a belt worn by a woman, or the lady of the house is the symbol of the other kind of power, the private and internal one, which is not less demanding than the public one, and likewise has little tolerance for resistance of individualism" (1998, 24). Such symbolism aptly underscores the struggle between private and public (royal) authorities, foregrounding their antagonistic relation. Royal and local authorities (whether familial or seigneurial) remain in conflict: the strengthening of the king's position could occur only under the condition of undermining the position held by family heads in "private" communities. Under feudalism, there was no political level of inter-group integration - no self-organizing political public sphere that could create and sustain a sense of common good in the highly 
differentiated motley of communities that constituted the state. At best, we can find legitimizing rituals and myths: the splendor of palaces and royal processions as well as ceremonious announcements of royal decisions performed - at least in cities - the function of generating shared sentiments and knowledge.

A similar role was played in the emerging Christian community by architecture and the design of churches as well as rituals and religious myths in general something that Gurevich (1985) has called the mediaeval "popular culture." The gravity, solemnity and rhythmical nature of collective prayer created a sense of a collective "We," although not necessarily a sense of the common good. ${ }^{353}$ Besides customary rituals and folk traditions, religion played a significant role in constructing a sense of local identity. It could be considered as the basic public sphere under feudalism: not only due to the social dimension of denomination (the community of believers, spending time together in church, participating in the same rituals), but also because of the excellent organization and standardization of message. The rhetorical exposition of religion, cultic objects (rosaries, sculptures and paintings ${ }^{354}$ ) and oral traditions (songs, lives of saints) was relatively homogenous and uniform, which helped to generate common knowledge.

The fluid boundary between the private and the public became a problem for authorities, both secular and religious. On the one hand, private communities noble families, local communities or heretical groups - appropriated public space, challenging authorities or seeking protection from them. As a result, entire villages were transformed into solidary neighborly assemblies closely tied with bonds of friendship. Abuses of power caused people to be wary of royal authority and curb it, apart from cases of particularly hideous crimes. On the other hand, power became privatized, dissolving in local communities. This affected not only the prince and royal officials but also the king himself; "his kingly powers came to be seen more and more as his private, personal, and hereditary property. This private appropriation of public power began at the top of the political hierarchy. As Fustel de Coulanges remarked long ago, publicus in ancient Rome meant that which belonged to the people; in Frankish Gaul, that which belonged to the king. The regalian power had become family property transmissible through copulation, through blood, to one's offspring; at each inheritance it was either divided among the children 'of the blood' or held undivided by a group of brothers, like a house. Slowly the palatium or palace in which the sovereign rendered justice

353 Especially if we take into account the fact that Latin was the language of the liturgy.

354 One needs to emphasize here the highly conventional and symbolic character of sacred art in that period. 
came to be seen as a dwelling place, affecting the meaning of certain words such as the Latin curia" (Duby 1988, 14). Just as "palace" became closed, becoming the king's private house, so the "houses" of all those who would partake in the royal power, even to a slight degree, became open to the "public," turning into palaces. Thus, the state began to resemble a private family organism, while family seats turned into "courts." By the same token, the boundary between the private and the public became blurred, bringing disintegration into the state community.

Private life - not the individual kind but the one lived together with family or neighbors, based on mutual trust - thereby acquires a special meaning. "In the late Middle Ages, we find the individual enmeshed in feudal and communal solidarities, incorporated into a more or less functioning system. As part of a seigneurial community or a clan or bound by ties of vassalage, he (or she) and his family moved within the limits of a world that was neither public nor private as those terms are understood today or were understood at other times in the modern era" (Ariès 1989, 1). It is a world marked by strong divisions that nevertheless have a purely personal or affective character. As Johan Huizinga points out, the late Middle Ages was a period of struggles between various parties. These conflicts, however, did not have any political or economic basis except for envy of possessions, goods and familial fame. "Racial pride, thirst of vengeance, fidelity, are their primary and direct motives" $(1987,21)$.

One humble harbinger of processes that would completely transform Western world in the next several centuries was that new groups developed within the feudal society - ones united by a sense of the common good. We mean here, on the one hand, the gradual emergence of chivalry with its specific ethos (Elias 2000; Ossowska 1986), and on the other - the rise of guilds, which were established mainly by merchants. Although these "conspiracies" were fought by the Church, which saw them as a danger to public order, they mainly focused on mutual assistance in financial and defensive terms despite being sometimes capable of imposing their own economic laws (Rouche 1992, 431-432). In both cases, we deal with the constituting of a collective "We" not at the level of the entire local community, but within its specific segments - ones formerly comprising specific "task groups." This process accelerated in the sixteenth century, which is generally considered to be the breakthrough point on the path towards modernity.

\subsection{The public sphere in the modern society}

Three parallel processes contributed to the transformation of the public sphere in the period between the seventeenth and the eighteenth centuries: the decay of 
traditional local communities (family, neighborhood or guild) integrated around a particular task or a joint fate; the growth of the state, which became increasingly capable of performing its functions due to the efficient monopolization of means of violence and management techniques; and finally, the emergence of new kinds of "private" communities or societé, which crystallized on the grounds of joint interests in circles gathering people of equal social status. All of these processes were combined, more or less directly, with the numerical and social growth of the "third estate."

The decay of traditional local communities is elaborated on in detail in the previous chapter. In the present context, it would be only necessary to note that the economic processes which led to the rise of the modern bourgeoisie were accompanied by the undermining of the material position of court circles. The ever more glaringly anachronistic character of the courtly lifestyle - excessive spending, exaggeration of honor, long chains of revenge - created an opportunity or even necessity for state intervention. In France, Richelieu forbade dueling under penalty of death, introduced luxury tax, and developed official lists of noble families, which allowed verifying claims of belonging to the nobility. In cases of family disagreements, it was also commonplace for the royalty to support one family member in his or her fight with another.

The consolidation of state power was related, on the one hand, to the economic boom in the sixteenth century, from which the state greatly benefited, and on the other - to the support of the third estate, which provided royal authorities with financial means and assistance, helping them to gain independence from court circles. The flow of money strengthened control over the executive apparatus: whereas public functions previously had, in a sense, the character of private activities (i.e. one would wield power in the name of the king, but with one's own means), the state extended complete control over the administration in the seventeenth and the eighteenth centuries. Processes of rationalization, described by Weber, were not only inspired but also largely realized and financed by the new social class.

Finally, the vital factor in the transformation of the public sphere was that representatives of the third estate would share not only their social standing but also broader interests. This is related not just to the group of entrepreneurs but also to the so-called middle class: "between the common people and the court was an intermediate zone, populated for the most part by people of culture, the minor nobility of office and the church, the middling sort of notables who in unprecedented fashion enjoyed staying at home and engaging in agreeable relations with a select 'society' (the very word that was used at the time) of friends" (Ariès 1989, $3-4)$. These circles formed, in the period from the sixteenth to the eighteenth 
century, a new kind of community: groupes de convivialité, within which a true culture of small associations was developed. In the eighteenth century, some of these groups transformed into statute-based institutions such as clubs or scientific societies, thus moving into the public sphere.

Jürgen Habermas strongly emphasized this process, making it a cornerstone of his communication-based model of the public sphere. Habermas draws special attention to the fact that socio-economic transformations were accompanied by the quick growth of institutions in the public sphere. Initially, club-like circles were places where literature would be discussed but with time they embraced political subjects. ${ }^{355}$ The rise of the novel created a community of readership, gathering people who would discuss currently published books. In Great Britain and France, cafés and salons began to be opened (the former on a mass scale at the turn of the seventeenth and eighteenth century), while in Germany "table societies" and literary clubs. The characteristic feature of most of these institutions of the public sphere was that they allowed, on equal terms, people of different social status: ones with roots both in the middle class and in the aristocracy. Moreover, those who participated in discussions hosted by such clubs did not consider themselves a closed group but a part of the broader public.

One of the important subjects addressed during such meetings was the role of the state in its relations with the "civil society." This last term would emerge (356 $^{356}$ in debates as a tool for articulating the growing differences between two modes of organizing social life: the social and the political order. More precisely, it ought to be said that it constituted an answer to the inclinations of absolutist

355 It is assumed that the revival of political criticism was facilitated by broad distribution of information in the press. Its birth was related to the development of capitalist economy in two ways. First, current information became valuable because it allowed choosing the most profitable market strategy. Second, by becoming a commodity, information came under the rule of supply and demand. The striving to maximize profits inclined the distributors of information to expand their clientele, contributing to the transformation of closed exchanges of letters into widely accessible papers of high circulation. With time, the press won some independence from the authorities and became not only a source of information but also a place where competing views would be presented, including the views of opposition (since the first half of the eighteenth century in Britain).

356 As we have already noted, the forming of the concept of civil society occurred, in England and France, parallel not only to the numerical growth of the third-estate "public," but also to processes of, first, breaking the power of aristocratic families, and second, monopolizing of power by monarchs, the latter famously expressed in the words of Louis XIV: "I am the state." 
state to control the actions of its citizens. As the Anglo-Saxon liberals put it at the time, it was an attempt at limiting the functions of royal power to the role of a "night watchman," while French republicans argued that monarchy ought to be abolished and replaced by a state that would express the "universal will." In other words, the concept of the civil society was employed, first, to prove that the monarch - being the incarnation of state - is not the only collective agent of social life, and to demonstrate that decisions of an alternative agent, which manifest themselves in the actions of citizens, receive a stronger legitimization (due to their collective nature) than the individual decisions made by the head of the state. Second, it was meant to define the duties of citizens to the society and its agency: the new liberal or capitalist state.

Thus, at the foundation of the citizenship, as understood at the time, we find the project of state not as the domain of monarchs and appointed officials, but of society, which becomes the sovereign. In its name, violence was monopolized in the form of law, and new directions of social development were defined. Both of these tasks were supposed to be realized by democratically elected representatives. The new state was to constitute a plane of integration not only of local or family communities but also of action-focused communities. In other words, the state would be obliged to create a universal social association, meaning that it would need to take responsibility for forming a public sphere that includes the entire society. ${ }^{357}$

However, the realization of this project at the turn of the eighteenth and the nineteenth centuries was preceded by fundamental changes in the social order, whose extent and depth were not acknowledged by the period's proponents of the civil society. In the process of shaping the modern society, individuals were uprooted from territorial and neighborly communities. Traditional ethos- and action-based associations would be dissolved too. Along with the consolidation of the power of the state, which embraced the duty of coordinating tasks previously realized by the above groups, their place was taken by legal and administrative categories that had the character of statistical aggregates rather than communities. With the passage of time, most of them selected their representatives in the form of professional associations, political parties and social organizations. However, they would represent interests or focus on tasks that remained

357 It is worth remembering that this new project of the public sphere was not - at least initially - a project of inter-group space, but of a space that would gather and integrate individuals; moreover, it would do so only with regard to those who fulfill specific criteria (qualification on the basis of wealth, education, sex etc.). 
on the margin of the state's interest rather than create holistic moral universes that would subordinate the actions of individuals to a good of a given social category.

One could therefore contend that, along with the development of the modern society, the public sphere was becoming more and more accessible. However, instead of becoming a space where common knowledge, sentiments and intentions would be recognized, it became an arena for playing social conflicts off against each other. It lacks any overriding idea that would integrate the various social groups that were forced to become part of the ever-stronger state. Let us recall that the fragmentation of the traditional society into task-oriented subsystems - a process that unfolded parallel to the development of capitalist economy in the period between the fifteenth and the seventeenth centuries - was accompanied by the diminishing of its religious legitimization. The religious community established by Christianity began to crumble under the pressure of the Reformation, while the cultural community - under blows dealt to the religious vision of the world by the budding science. ${ }^{358}$ Nevertheless, the scope of achievements during the Enlightenment was too narrow to replace religion in its community-forming function. In other words, in the initial phase of the development of the modern society there was no clearly articulated idea that could lend internal meaning and coherence to the emerging new form of organization of social life and a sense of a collective "We" to the internally fragmented society. However, at the turn of the eighteenth and the nineteenth centuries, nation became one such idea.

Just like in the case of the concept of society, it is widely debated whether nations exist objectively or are only subjective categories. ${ }^{359}$ Since the publishing of Benedict Anderson's seminal work (2006), sociology began to emphasize the "imagined" character of such communities, which does not change the fact that they are firmly rooted in linguistic and territorial collectivities consolidating already since the eleventh century (cf. Zientara 1985). It is in relation to this type of collectivities, originally called "peoples," that Walter Żelazny (2004) uses the term "ethnic community," indicating at the same time that the concept of nation is born when one ethnic group gains political dominance over other groups living on the territory of a given state. The "forging of nation" as a community,

358 Many authors emphasize that the printing press has had a tremendous significance for both processes. According to John B. Thompson (1995), this invention radically changed the social organization of symbolic authority, giving rise to processes of its fragmentation, which have reached their zenith in modern societies.

359 Cf. the survey of views on this issue in the book by Walter Żelazny (2004), especially Chapter Three. 
and the role it played in the consolidation of nineteenth-century states, are perfectly captured by Anderson, which makes it unnecessary to address these issues at length here. We shall only emphasize that a significant role in this process was played not only by the invention of the printing press and the resulting homogenization of ethnic languages, which later supplanted Latin as the language uniting the religious community (a factor of crucial importance to Anderson), but also by the intensification of communication among people in the broadest sense of the term, including the development of postal services, roads, means of transport, etc. (cf. Thompson 1995).

The nineteenth-century state was, therefore, more national than civic in the traditional sense of the latter term. It created structures whose goal was to unify the nation. Among them a key role was played by schools, which were to promote a coherent model of civic attitudes by standardizing language, knowledge and manners, disciplining bodies, restraining dialects, and correcting accents. Between the consolidating structures of state and law on the one hand, and individuals and "private" communities (families, ethnic communities, professional associations etc.), on the other, a new kind of public sphere emerged. It would mediate between the level of the state and the level of various groups.

Moreover, it is important to point out that nation, despite its crucial position, was not the only subject of so-called secular religions, which began to emerge with time in the modern society. As Serge Moscovici writes, "the idea of them was one of the discoveries of the French Revolution, and Robespierre was the first to see them as a powerful way of regenerating a nation and an instrument enabling him to make a Republic replace the monarchy completely. Secular celebrations of Reason and the Supreme Being crowned the discovery" (1985, 354-55). ${ }^{360}$ Various ideologies developed in the nineteenth century (and in the

360 It is highly characteristic, however, that the leaders of the French Revolution referred to the concept of the citizen rather than nation, and that the search for an appropriate "secular religion" could sometimes assume caricatured forms. In a fascinating essay, Lynn Hunt indicates that the concept of the public has been rapidly expanded: "Dress, language, attitudes towards the poor, providing jobs in the city and on the land - all had become gauges of patriotism. What separated the private from the public man?" $(1990,14)$. Leaders of the Revolution were constantly worried about attire; endless projects of "uniforms" for citizens were developed, or at least ways of "marking" them as participants in the new space uniting all patriotically-minded Frenchmen. Since 2 July 1792, the Convention legally obliged all men to wear a tricolor bow; after 3 April 1793, the law was extended to include women. In May 1794, the Convention demanded from Deputy David, a painter, that he design a national outfit. 
first half of the twentieth) elevated different kinds of collectivities to the rank of the "Supreme Being": the working class, white people (whose cult we find in fascism), and the middle class as a group that combines all virtues necessary for the functioning of the civil society.

Just like regular religions, secular ones have three main goals. First, as Moscovici argues, "they create a total view of the world as a palliative to fragmentary and divided nature of all science and technology and indeed of knowledge in general"; in everyday life, he claims, people need "an overall view with a single cause (social class, race etc.), a universal principle (the class struggle, natural selection, etc.) and a definite picture of the human and non-human world. What secular religions essentially do is provide us with total view of that kind. They offer us a concept of the world in which every problem has its solution" (355). While maintaining an appearance of scientific approach - by retaining appropriate language and arguments - they recombine its elements, focusing primarily on social reality.

The second function of secular religions is to provide a moral code that allows to "harmonize relations between the individual and society and to reconcile the social and antisocial tendencies present in the former" (356). The only way to reach both of these goals is to socialize individuals in such a way as to make them identify with the collectivity. In this context, Moscovici recalls Freud, who argues that there are only two ways for a community to hold together: the use of violence or the creation of emotional bonds between individuals and community. We could just as well refer to the theory developed by Greenspan (presented in Chapter Two), which demonstrates the role of such bonds in socializing individuals.

Finally, the third function is to hide a mystery. "Each religion has its own. In its name it imposes rules and proclaims truths it does not explain" (357). Revealing it by some community member would spark violent emotional outbursts among others, making them panic disproportionally to the revelation. As Moscovici emphasizes, "most artificial crowds (armies, churches, parties) are in touch with a mystery of that kind. They have a whole range of ceremonies, emblems and passwords (one need only think of the freemasons) that protect it and attack any attempt to reveal it" (357). Such mysteries are meant to consolidate hierarchies and rituals developed around them, and to create degrees of initiation, whose gradual achievement determines the position of the individual in the community.

The process of replacing traditional, sacred visions of the world with images created by "secular religions" or ideologies was accompanied by a significant change. One of the crucial differences between the two kinds of the sacred is 
the shift of accent from rituals meant to control nature, and order actions taken with regard to it, to rituals whose goal would be to primarily gain control over the social world and the relations between ever more numerous communities, usually of conflicting interests. This fundamentally changed the very concept of the common good whose establishing is served by these rituals. Whereas during tribal rituals the entire community could unanimously pray for rain and bountiful crops, in modern societies rain would become an "ambivalent" phenomenon, depending on what consequences it might have for farmers, stock market players betting on bad crops, or city-dwellers dreaming of sunny weather.

Among those secular religions, the one organized around the nation performed a special function: it was meant to subordinate the goals of individual communities - ones based on ethnicity, class or religion - to the superior goal of the national community, whatever it would be. In a diversified modern society, the interest of the nation is supposed to be exactly that which creates the common good. "Dying for one's country," Anderson notes, "which usually one does not choose, assumes a moral grandeur which dying for the Labor Party, the American Medical Association, or perhaps even Amnesty International cannot rival, for these are all bodies one can join or leave at easy will" $(2006,144)$. Even when the goal is not to die but to live for one's country, invoking the national interest is an argument that cannot be possibly challenged by the interest of any, even most distinguished group.

\subsection{Contemporary transformations of the public sphere}

In the historical dimension, the creation of the modern public sphere is connected with processes that are well-documented by social sciences: the monopolization of violence, breaking of local communities' power, the rationalization of management, the development of administration capable of registering and controlling the growing population, the standardization of language and knowledge and the increase in efficiency of communication. At the foundation of these processes, we find not only technological progress but also the development of sciences, including social sciences that objectivize the mechanisms of collective life and provide the "society," or rather its individual members, with feedback about their behavior, attitudes and views.

This kind of community - organized around the concept of nation, certainly not without difficulty - began to crumble in the second half of the twentieth century. According to more and more scholars, one of the most characteristic features of our times is none else but the crisis of the nation state, the most general kind of community developed so far, one determining both collective and 
individual identity. This process is supposed to be the consequence of synergy between many processes transforming contemporary life, which are usually gathered under the umbrella term "globalization." As this word suggests, the main feature of these changes is the emergence of a new type of territorial unit, one that establishes the basic framework for economic, political, social and cultural processes on the scale of the globe, not on that of the state (Castells 2001a).

One effect of the breakdown of the significance of the national state as the basis for coordinating individual and collective actions is also the collapse of the capability to integrate and mobilize society through the existing public infrastructure: parliamentary elections, organizations of the "civil society," or organizations representing the interests of various groups (trade unions etc.). The impression is that - just like in feudalism - the public sphere is privatized, displaying unclear and fluid boundaries between the private-turning-public (e.g. in the form of popular reality shows) and the public-turning-private (as in the case of ties between politics and business). This resembles the twilight of the Middle Ages, as described by its historians. One could also say that the trends culminating in the emergence of the kind of public sphere that was characteristic for societies of the modern type, reached their apogee and then froze in a form that increasingly often diverges from ongoing processes of social life.

The first of these trends was the universalization of the public sphere, which extended its reach to encompass more and more diversified groups. At the same time, it has had to become increasingly abstract, losing one important feature the real experience of coexisting with others. The tribal assembly could physically gather all the elderly; today's voting, in turn, takes place in isolation. Although each voter knows that many more people are doing the same thing on that day, and that they act in accordance with the same practical knowledge he or she has (i.e. about polling stations, candidates, ways of voting etc.), there is no physical possibility of feeling a part of the voting community, all the more so since all participants are also conscious of their vastly differing political inclinations and the conflicts this entails. ${ }^{361}$

The universalization of the public sphere was achieved thanks to the development of communication technologies. On the one hand, without mass media there could be no democracy for all; on the other, however, the dependence on

361 It is worth pointing out that this trend is not limited to the sphere of politics. Its counterpart in the area of economy is the global market along with the abstract medium of money, while in the area of collective representations - the global village, along with its universal visual code (film), as first described by McLuhan. 
media has vital consequences for processes of creating and sustaining common knowledge. The image of society in the broadest sense - as a "meta-group" to which we belong - cannot be possibly developed on the basis of direct experience. There are no real spaces where sentiments, intentions and knowledge would be made common. Although we know that many others are doing the exact same thing at a given moment - such as taking out money, buying food, voting, watching news on television - we do not have direct contact with them. We have no access to their reactions and we cannot observe their behavior. We can only learn through media about the collective effect of those actions in aggregated form as voting results, dwindling of bank reserves, or the rise of food prices. The only space in which individuals learn about each other, their views and behaviors, is the space of mass media. They produce the image of an abstract "society" to which we belong. Ultimately, the task of creating and promulgating of this image has been taken by the media industry, with all the consequences this entails.

The more abstract and separated from real experience the "meta-group" becomes, the more it is exposed to the risk of fabrication. As a result, "[ $t]$ he autonomous citizen, whose reasoned judgement and participation was the sine qua non of the public sphere has been transformed into the 'citizen consumer' of packaged images and messages, or the 'electronic mail target' of large lobbying groups and organizations” (Benhabib 1992, 44). In other words, instead of contributing their "private" social or cultural resources to the public sphere, its participants are to an increasing extent constructed, mobilized and managed by major commercial or political players.

The second trend, closely connected to the first one, is the progressing abolishing of "particularities" - the more individuals (social groups) the public sphere includes, the more abstract and general their shared identity has to become, the more standardized (shareable) the language of communication and common knowledge have to be, and the subtler (more civilized) their behavior has to be. One could therefore say that it is not only the community but also the individual that becomes more and more abstract. The more individualized we are, the more difficult it is to "reveal" oneself and come to existence in public space as a specific, particular person known by name and surname. The logical complement to universalization is the blurring of the boundary between private life-worlds: an autonomous subject equipped with individual rights cannot be a member of a community as long as it means being-shared-with-others and thus, being dependent on others. ${ }^{362}$

362 As Tönnies intuited, it is the reflexive identity that is artificial, general and non-unique. Here we encounter a harbinger of the postmodern paradox: one's authenticity cannot be constituted reflexively (cf. Chapter Four). 
The third trend, which results from the elimination of embeddedness in a "private" community, is the generalization of the concept of the common good. In modern societies, the integration of the population around the concept of nation represented by state subordinated any particular common good to the abstract "reason of state," detached from particular conditions of individual and collective life. Along with the crisis of the nation state, we observe the dissolving of that which could mobilize individuals to take coordinated action. If it is not a common good for a particular community, only the abstract good of humanity remains.

All of these processes have contributed to the emergence of contemporary society, one no longer comprised of communities but of individuals - a society in which the state is "omnipotent," while local public spaces have lost their intrinsic meaning and sense. The politicization of social life has caused the private sphere to shrink down to the intimate sphere, while public space has become a "no-man's land." One could stroll in a park without feeling any sense of unity with passers-by, while some people would be even afraid of others, or at least annoyed by their dogs or children. Negotiations between collective actors no longer express differences of opinions held by members of society. Parties and organizations, which were supposed to represent the interests of citizens, are actually beyond their control, and are playing their own game with the public administration. Results of elections are no longer a derivative of how society's opinions are distributed, but they largely constitute an effect of rhetorical efforts made by politicians. What is more, the latter do not even attempt to convince citizens to embrace their views or understand them better - they merely try to mobilize short-term support during elections.

This fragmentary description, which gathers the most frequently voiced opinions, meant to support the thesis about the crisis of the public sphere (regardless of whether we accept this thesis or not), suggests one thing, namely that the political order that used to determine the shape of public life is currently drifting apart from the social order. More and more frequently, the latter assumes the form of a dis-order, thereby becoming a symptom of deep transformations occurring under the surface of the public sphere. 


\title{
CHAPTER EIGHT. MECHANISMS OF GREAT TRANSFORMATIONS
}

\author{
[...] every working social scientist must be his own \\ methodologist and his own theorist.
}

C. WRIGHT MILLS, The Sociological Imagination

Sociology is by definition a historical discipline insofar as its basic object of study is society, which is understood as forms and constellations of human relations subject to a constant change. This does not necessarily mean that sociology cannot be a strictly theoretical discipline, nor that it is doomed to rely in its inquiries on accounts of individual cases. Nevertheless, the opposition between theory and description, between nomothetic and idiographic sciences, which lies at the foundation of the development of modern sociology and seems to motivate it to this day, is widespread to the extent that requires closer examination.

The above opposition has inspired attempts to either make sociology more "scientific," following the classical model of natural sciences, or conversely (though to a much lesser degree) - give it the status of a unique discipline employing in its research an original and highly specific mode of understanding. These tendencies themselves showcase the historical character of social phenomena, which naturally include the development of all sciences. However, it seems even more important that they are rooted in nineteenthcentury dilemmas ${ }^{363}$ and fail to account for real changes occurring in science. $^{364}$

363 This is yet another argument in favor of the thesis formulated in Chapter One, namely that the major directions of development in modern sociology have been determined by the vision of society produced by nineteenth-century sociologists and then taken for granted as universal.

364 This was emphasized by Edmund Mokrzycki, who wrote that at the foundation of contemporary sociology we find the methodological model that was adopted in the 1930s as the only scientific one; however, "it had been already deemed inadequate and abandoned by the discipline that formulated it, namely the philosophy of science" $(1980,8)$. 
The nineteenth century was, on the one hand, a time when classical science triumphed, culminating in Newtonian mechanics, and on the other - a period when this vision of reality encountered strong opposition, when voices were raised against the view of the world as passive, lifeless and governed by universal laws of causation that operate regardless of the objects they affect. In the domain of culture, this opposition manifested in the form of the Romantic rejection of Enlightenment rationalist ideals, while in the humanities - as an emphasis on the ineradicable difference between the world of nature and the world of humanity. It is the underlining of that difference that gave rise to the many variants of "interpretive sociology," which assumes - as Wilhelm Dilthey does - that "man creates a world of history within the world of nature, an empire within an empire, one in which values take precedence over causes, freedom over necessity" (Szacki 2002, 415).

However, neither Dilthey nor Rickert - or Weber, the "founding father" of "interpretive" or "humanistic" sociology - would place their own methods in opposition to the methods of natural sciences, treating the former rather as complementing the latter. One could put it even more strongly and argue that the philosophy of science to which these thinkers subscribed, especially with regard to its role in explaining both organic and inorganic matter, was basically identical with the theories dominating in natural sciences, while "interpretation" was meant to introduce a certain "additional value" constituting "a procedure of revealing what is hiding under the surface of phenomena observable thanks to methods employed by natural sciences" (Szacki 2002, 423). It was to be a procedure of furnishing these phenomena with historically changing meanings.

From this perspective, the work of those who founded "humanistic" sociology was part of more general efforts made by researchers in that period, including those operating in the natural sciences. After all, the opposition to the vision of the world established by classical science did not regard its methods but rather assumptions lying at the foundation of its theorems. Among them a fundamental role was played by the conviction that "at some level the world is simple and is governed by time-reversible fundamental laws" (Prigogine \& Stengers 1984, 7; emphasis preserved). This, in turn, led to the formulation of the belief one widespread today not only among amateurs but also among some social researchers - that the aim of science is "discovering the unique truth about the world, the one language that will decipher the whole of nature [...] the fundamental level of description from which everything in existence can be deduced" (52; emphasis preserved). 
As Prigogine and Stengers have demonstrated, the major achievement of modern science was to overrule these assumptions. ${ }^{365}$ The world of today's natural sciences is a complex one in which the irreversibility of certain processes "lies at the origin of most processes of self-organization" $(1984,8)$ and is a rule rather than exception. It is a world where randomness and fluctuation are more frequent than cases of determinism. This shift in assumptions did not stem from any arbitrary decision. "In physics it was forced upon us by new discoveries no one could have foreseen. Who would have expected that most (and perhaps all) elementary particles would prove to be unstable? Who would have expected that with the experimental confirmation of an expanding universe we could conceive of the history of the world as a whole"? (8-9). These discoveries did not annul past ones, but revealed their limitations, demonstrating that apart from reversible processes independent from the direction in which time flows, which were the main subject of enquiry in classical science, there do exist irreversible processes that are dependent on that direction. ${ }^{366}$

Let us dwell for a moment upon this distinction, as it carries vital consequences, also for the way we conceive of social phenomena. To understand this distinction, it becomes crucial to grasp the difference between processes occurring in the state of equilibrium and those occurring otherwise, which are designated by the term "dissipative processes." 367 "Matter near equilibrium behaves in a 'repetitive' way. On the other hand, far from equilibrium there appears a variety of mechanisms corresponding to the possibility of occurrence of various types of dissipative structures" (13). In other words, lack of equilibrium does not necessarily entail a state of chaos, but is rather the kind of a dynamic state of matter that reflects mutual influences between system and environment. The emerging structures of order are created as a result of "communication" between individual

365 It seems characteristic that Prigogine and Stengers consider nineteenth-century sociology to be a discipline whose emphasis on processes of becoming, and focus on increasing complexity contributed to the creation of conditions favoring the change of paradigm in classical science $(1984,12)$.

366 The role of the criterion allowing for differentiation between the two kinds of processes was given to the concept of entropy since the only reason for an increase in entropy is the operation of irreversible processes.

367 Ilya Prigogine, co-author of the book referenced here, was awarded the 1977 Nobel Prize in chemistry for work on dissipative structures created as a result of non-linear processes in far-from-equilibrium systems. 
elements in the system. "In addition, the type of dissipative structure depends critically on the conditions in which the structure is formed" (14). ${ }^{368}$

Reading such statements, one could only repeat after the quoted authors that " $t]$ he analogy with social phenomena, even with history, is inescapable. Far from opposing 'chance' and 'necessity', we now see both aspects as essential in the description of non-linear systems far from equilibrium" (14). Paradoxically, however, that which may be obvious to representatives of natural sciences or philosophy of science proves difficult to accept in the contemporary sociology. The acknowledging of the historical character of social phenomena still bears associations with nineteenth-century historicism, incidentally interpreted in terms entirely contradicting the intentions of its representatives. ${ }^{369}$ As a theoretical ideal, this approach posits a set of universal laws describing linear dependencies unaffected by the passage of time.

It is easy to demonstrate (as has been often done) that this kind of a "sociological frame of mind" was shaped under the influence of neo-positivist vision of science. However, it remains much more difficult to answer the question why this particular influence has held sway to this day. ${ }^{370}$ One of the reasons could be - as was aptly noted once by Edmund Mokrzycki - that sociological practice

368 One of the more interesting moments in the creation of dissipative structures is the reaching of the so-called point of bifurcation, where systems begin to undergo considerable fluctuations. "Such systems seem to 'hesitate' among various possible directions of evolution, and the famous law of large numbers in its usual sense breaks down. A small fluctuation may start an entirely new evolution that will drastically change the whole behavior of the macroscopic system" (14). The concept of bifurcation resembles - in its general outline - the well-known psychological theory of the conflict between pursuit and evasion, formulated by Neal E. Miller (1959).

369 The existence of so-called historical sociology (cf. Morawska 2002) in no way disqualifies this statement. First, this approach so far constitutes only a margin of sociological research. Second, many works assumed to represent this current simply utilize all assumptions made in modern sociology to analyze phenomena or societies only slightly removed from us in time. Sociological mainstream was never impacted by the assumptions that lie at the foundation of this approach. This is confirmed by the distanced (if not indifferent) respect with which scholars regard works by Norbert Elias, one of the creators of this approach, who is often quoted (just like other classics) but rarely treated as a source of inspiration.

370 To see that sociological thinking is still shaped by this vision of science it suffices to take a look at introductions to handbooks of methodology. For example, the one by Chava Frankfort-Nachmias and David Nachmias argues: "The most basic assumption of the scientific approach is that there is a recognizable regularity and order in the natural world; events do not just occur" (2008, 5; emphasis added). 
is "based on a tripartite division of the discipline into methodology, theory, and empirical research" $(1980,269)$, with the three dimensions rarely, if ever, communicating with each other. Thus, sociology would lack the kind of positive feedback between theory and actual research that caused physics to move from classical mechanics to contemporary theories. Regardless of the causes, however, it is true that sociology has been facing a growing crisis since the 1960s, as it has kept encountering more and more obstacles in explaining social processes given the increasing pace of changes that deeply transform contemporary society.

As we have demonstrated earlier, one of the symptoms of this crisis is the de-theorization of the very concept of society. Meanwhile, as we wish to argue, it is the study of the historical changeability of forms taken by human relationships that plays a crucial role in grasping what society is. At this point of the present discussion, it is necessary to search for a more general model that would, on the one hand, help provide a more adequate description of the very process of historical changeability, and on the other - make it possible to answer the question about the distinctive features of that form of society which is currently emerging from these transformations (if it in fact does). We do not seek to find one universal cause or principle governing the development of human societies. ${ }^{371}$ The point would be rather to isolate those components that could be considered - from the perspective of a theoretical model proposed in earlier chapters - as factors that perhaps may not be directly causing but at least significantly contribute to the historical changeability of the subsequent forms of social organization.

\subsection{Dynamics of change}

At the foundation of sociological differentiation between various kinds of societies, we find the question of the mode of organization specific to a given social order, or in other words: the dominant principle of coordinating collective actions. As we have indicated, this question - one that established sociology as a distinct scientific discipline - emerges at the moment of transition from the traditional order to the modern one as a reaction to the accompanying breakdown of former norms of behavior and the consequent disorganization of social

371 Such an approach to explaining the changeability of human societies is explicitly contained in a strong claim made by Giddens, who argues that "the search for a theory of social change [...] is a doomed one", simultaneously adding that " theory' means in this instance explaining social change by reference to a single set of mechanism, such as the old evolutionary favorites of adaptation and differential selection" (1986, xxviii; emphasis added). 
life at all its levels. A direct expression of such a reaction is the assessment made by Durkheim, who concludes his discussion of two types of social solidarity by writing about the society of his time: "Our beliefs have been disturbed. Tradition has lost its sway. Individual judgement has thrown off the yoke of the collective judgement. On the other hand, the functions that have been disrupted in this period of trial have had no time to adjust to one another. The new life that all of a sudden has arisen has not been able to organize itself thoroughly. Above all, it has not been organized so as to satisfy the need for justice that has been aroused even more passionately in our hearts" (2013, 317-318).

Taking into account what has been already said about the transformations of human societies since the moment of "foundational holism," one could argue that this "disruption of functions" characterizes all periods of transition from one form of sociality to another. As we have shown, the very act of "foundational holism" can be interpreted as a specific reaction to the upsetting of actions previously controlled by biologically conditioned habits, which was caused by the emergence of early human psycho-culture. Similarly, the period of a specific "anomie" or the domination of "direct regulation" based on physical force, as Norbert Elias calls it (2000), occupies the place between the post-tribal form of sociality, and the one characteristic for a fully-developed system of European feudalism. Finally, it was not only Durkheim but also a majority of intellectuals of his time who emphasized the sense of being lost or disorientated that accompanied the transition to modernity. Karl Marx put it in similar terms: "Constant revolutionizing of production, uninterrupted disturbance of all social conditions, everlasting uncertainty and agitation distinguish the bourgeois epoch from all earlier ones. All freed, fast-frozen relations, with their train of ancient and venerable prejudices and opinions, are swept away, all new-formed ones become antiquated before they can ossify. All that is solid melts into air, all that is holy is profaned" $(1988,212)$.

These periods of transition are usually linked to the change of "production forces" or tools used by people in their efforts to secure means of living. It is not only Marx who emphasizes the significance of this factor for the alteration of forms that organize social life. Most contemporary theories of social change do the same, whether they are formulated in sociology, anthropology or economy. One of the more frequently used categorizations - i.e. one distinguishing agrarian, industrial and post-industrial societies - owe its popularity not only to a renewed interest in technological changes after the publishing of the study by Daniel Bell (1973), which proclaimed the arrival of post-industrial society, but also to the universally shared conviction that changes of this kind have had the broadest influence on other realms of social life. It is in this spirit 
that Manuel Castells justifies the significance of information technologies for the transformations occurring in contemporary world (2001ab). He emphasizes that they affect changes of social structures, relations of power, transformations of family, experiences of individuals etc., simultaneously contributing to the formation of a new kind of social order and a new type of culture.

Castells rejects the accusation of "technological determinism:" after all, as he aptly observes, to point out the impact of the transformation of tools on the transformation of forms of social organization is not necessarily to negate other factors contributing to these processes (cf. 2001a, 5-13). He uses the example of China, which already in the fourteenth century had at its disposal, in a working form, all the inventions whose application later triggered the Industrial Revolution in the West. ${ }^{372}$ That this revolution never happened in China was - in his view - the result of a strong-state policy. However, the fact that Japan played a key role in the IT revolution of the twentieth century was also, as Castells emphasizes, the effect of actions taken by the state. Therefore, his acknowledgement of the role played in each case by the state boils down to the argument that, depending on historical conditions, the state either holds back or stimulates social transformations. It is difficult to accept this as a satisfying explanation.

The case of China is one of many examples that clearly illustrate the Eurocentrism of various theories of social change, i.e. their disregard, discernible already in their fundamental assumptions, of the "evolutionary" dissimilarity of non-European societies. Another example is provided by various concepts of modernization developed since the 1950s. They usually assume that the transfer of technologies and patterns of production from more developed countries to "backward" ones would cause desirable changes in the latter's organization of social life, turning them into fully developed societies. ${ }^{373}$ One of the

372 “Thus, around 1400, when European Renaissance was planting the intellectual seeds of technological change that would dominate the world three centuries later, China was the most advanced technological civilization in the world" (Castells 2001a, 7). Castells refers to analyzes by Joel Mokyr contained in the 1990 study The Lever of Riches: Technological Creativity and Economic Progress (New York: Oxford University Press).

373 This assumption is found at the basis of the communist experiment, which - generally speaking - constituted an attempt at accelerated modernization of a country located on the peripheries of the modern world. Clearly, Lenin's famous slogan ("communism is Soviet power plus the electrification of the whole country") can be considered a synthetic (and, as it later turned out, not fully adequate) rephrasing of two key elements of modernity: technological progress and democratization of social life. 
reasons for the inadequacy of such theories is certainly the fact that - as Jürgen Habermas rightly observes (1998) - "[ $\mathrm{t}]$ he theory of modernization performs two abstractions on Weber's concept of 'modernity'. It dissociates 'modernity' from its modern European origins and stylizes it into a spatio-temporally neutral model for processes of social development in general" (2).

It is also possible to argue that the emphasis on technological changes as the driving force of the evolution of human societies is supported by the turbulence and a specific "total character" of two technological revolutions, of which the first (Industrial) has formed the modern kind of social order that prevails in Western countries, while the second (Information) is currently transforming that order. It is also not without merit that the expansion of Western countries, which began in the seventeenth century, entailed imposing on other states the kinds of lifestyles and modes of social organization that are characteristic for industrial civilization. However, regardless of the reasons for highlighting technological transformations, the emphasis placed on the development of tools makes it hard to see that periods of transition from one form organizing social life to another are characterized by a co-occurrence of many interdependent processes whose unique combinations could yield similar or entirely dissimilar effects depending on social conditions.

Naturally, we are unable to thoroughly analyze these dependencies. Still, we wish to draw attention to several of them - ones that seem to accompany most periods of transition predating the emergence of those distinct forms of social order that have been identified in social sciences, and whose existence is referred to in more or less systematic way in the present work. Let us note at this point that we take into account five such forms: tribal societies, whose characteristic feature is the dispersion of power and the flattening of social differentiation; ancient empires or "agrarian bureaucracies" characterized by centralized power, poorly developed land ownership and an elaborate system of redistribution; feudal societies, typically exemplified by mediaeval Europe; ${ }^{374}$ modern societies identified with that kind of social organization which found clearest form in nineteenth-century Western Europe; and finally, the postmodern society - a term that refers to imprecisely defined peculiarities of the currently emerging organization of social life. In all of the above cases, during periods of transition

374 As we have already mentioned, the first three slightly different forms organizing social life are usually referred to with the term "traditional order." We shall return to this term further on, but here - due to examples we shall use - we retain this differentiation for the sake of convenience. 
from one social form to another, there emerge several factors that fuel the process of change.

The first factor is linked to large-scale movement of people, either between populations or within them, that causes locals to mix with migrants. The rise of ancient empires was correlated with the influx of slaves from conquered territories. ${ }^{375}$ The emergence of the feudal society was preceded by large-scale barbarian migrations that completely rearranged the ethnic map of Europe and large parts of Asia. The growth of the modern society was accompanied by a relatively massive migration from the countryside into urban areas. Today, as we are more and more frequently speaking of the development of a postmodern society, we witness an intensified migration from South to North, which some scholars call the new Great Migration of people.

The second factor is linked to the fact that movements of people were tied to the relative exhaustion of resources in habitats that constituted the former dwelling place of migrants. According to current hypotheses, the factor that triggered the barbarian migration was the abandoning of their former habitat by Mongolian tribes, which was caused by the depletion of pastures in Far Eastern Asia. The sequence of westward movements initiated in this way not only put an end to the rule of Rome but also gave rise to the development of European feudal society, which thus emerged directly from tribal organization. ${ }^{376}$ Migration from the countryside to urban areas in the period preceding the Industrial Revolution was caused by a radical decline in the capability of rural communities to feed their population, which resulted from repeating periods of poverty and hunger as well as the opening of new possibilities in the growing cities. At the foundation of currently observable migrations, we also find a similar process: populations from poorer regions of the so-called South are drawn by the immensely richer countries of the North. ${ }^{377}$

375 In the case of empires, the flow of people, though certainly less intense, would also occur in the other direction, e.g. from the center towards the peripheries, as necessitated by the establishing of garrisons and trade outposts on conquered territories.

376 The specific Eurocentrism of history, especially its popular version, has caused the popular imagination (from which most sociologists also derive their notions of historical processes) to usually conceive of European feudalism in terms of an "heir" to the Roman Empire rather than to the tribal form of social organization. The latter's impact on processes that led to the rise of feudal organization is emphasized by Norbert Elias (2000). More recently, it has been underlined by Modzelewski (2004).

377 However, as Jacek Kochanowicz has brought to our attention, today's China witnesses the complete inverse of this pattern of migration, which is related to the fact that the wealthiest regions of this state are located in the south. 
The third factor is that periods of transition are also preceded - in strict connection with the growth of population - by the exhaustion of the sources of social power, i.e. those resources whose use constitutes the basis for the expansion of a given form of social order. In the case of tribal and imperial societies, it was territory. Its expansion, however, ultimately had to meet with resistance from neighboring populations. In feudalism, this resource was land, granting of which set up a specific relation between the sovereign and vassals. Finally, the functioning of the modern order, in which economy is based primarily on the mining and food-processing industries, depends on access to natural resources. ${ }^{378}$

As Norbert Elias accentuates in his analysis of the functioning of the European feudal system, "[t]he smaller society's possibilities of expansion became, the larger grew the reserve army from all strata, including the upper stratum" (2000, 238). On the one hand, this stimulated a search for new career paths, which began to modify - as if from the inside - the system's modes of functioning, as in the case of the concentration of knights around courts, development of trade, and the growth of state bureaucracy. On the other hand, it favored seeking out and accepting those "technological" solutions whose application would either contravene the system's logic of functioning, or significantly contribute to its change. ${ }^{379}$

This leads to the fourth factor, namely the emergence and consolidation of groups whose existence within a given social order was not legitimized by its main principle of organization, and whose functioning would not be regulated in the same way as other categories of population. In traditional societies, this group was comprised by city-dwellers, while in the modern one - by the so-called intelligentsia. ${ }^{380}$ It is not an accident that these groups played a leading role in the transition to the new kind of social order. Already Max Weber indicated

378 One crucial factor in the consolidation of this kind of social order in Europe was the conquest of overseas territories, which not only extended access to resources, but also provided an additional career path for representatives of various social groups whose development was previously thwarted.

379 The use of new, electronic means of communication facilitates the transfer of production to those regions of the world where workforce is cheaper, although this simultaneously undermines the logic of the modern social order, which relies on the concept of competing states.

380 This pattern is less clearly visible in transitions related to the traditional order, although one can observe that there are no distinct groups of priests in tribal communities though there are people performing religious tasks. These groups began to play a key role only in ancient empires and in the feudal society. 
that "major forerunners of the modern, specifically Western form of capitalism are to be found in the organized urban communes of Europe with their particular type of relatively rational administration" $(1978,240-241)$. On a similar basis, one could demonstrate that the role ascribed in the postmodern society to "experts" (Giddens 2006) or "symbolic analysts"381 (Reich 1992) has its roots in the functions (not fully crystallized back then) performed in the modern society by the intelligentsia.

Each of those factors alone contributes to the destabilization of a given form of social order, but their joint occurrence has to lead to the complete dissolution of its network of connections. Let us note that the above phenomena, discernible in transition periods, share one feature, namely - they introduce significant, largescale and illegitimate modifications to interactions occurring at various levels of social life. They are illegitimate for three possible reasons. First, they constitute the effect of collisions between actions inspired by extremely dissimilar visions of reality. Second, they introduce new, incompatible elements into these visions as a result of individual- or group-led quest for new means of expansion. Third, they express different lifestyles developed by groups that were hitherto marginal in a given social order. All of this causes the destruction of not only the former "repeated assemblies," which distinguished a given type of social order, but also its collective representations that lay at the heart of its characteristic form of sociality.

At the same time, the very similarity of factors operating in transition periods suggests the existence of three more general processes forming a basis for the evolution of human societies.

The first process is related to the numerical growth of population, which started when people adopted a settled lifestyle. We are far from subscribing here to some new version of Malthusian theory, but merely wish to draw attention to the fact that population growth in individual societies - regardless of whether it would occur through biological reproduction or by "co-opting" people from other countries or territories as a result of conquests or migrations - is a factor that autonomously leads to their differentiation and enforces changes in the organization of social life. These include, on the one hand, political transformations

381 Other names have been coined too. For example, Peter F. Drucker (1994) uses the term "knowledge workers," while Manuel Castells calls them "innovators." The latter term ties the significance of this group to the transition from post-industrialism to the "information era," i.e. the system in which processing information is the economy's key aspect. 
stemming from the necessity to solve problems arising from the "management" of growing populations, and from contacts with neighboring collectives, leading to the emergence of separate groups and institutions performing specific tasks linked to the above issues, first within communities and later on higher levels of social integration. On the other hand, these include economic changes arising from the fact that the emergence of these kinds of groups and institutions either leads to or results from the fact that they accumulate a major portion of those resources available to the collectivity which determine its power in contacts with other communities. ${ }^{382}$

The differentiation of a given collectivity has consequences of a strictly social character as well, because it leads to the emergence of new types of groups: no longer "natural communities" (ones tied with blood bonds) but ones produced by certain features of social position, which create a community of interests, lifestyles or values. A classic example of such communities is provided by the estates of the feudal society or the classes of the modern society. Let us note, however, that the community of interests can be also established on different grounds than a similarity of lifestyles or relations to means of production. It can stem from the exchange of "services," as in the case of ties linking feudal lords and their peasants ("I defend you and you feed me") or paternalistic relations between factory owners and their workers ("I give you work and you work for me"). It can also emerge from alliances formed in the face of danger posed by a shared enemy (real or imagined), or from combining resources necessary for expansion, either in terms of territory or capital. Finally, it can be created on the basis of a shared ethos (chivalry, guilds, professional associations) or shared views (parties, scientific schools), which surely does not preclude more pragmatic actions.

Those new kinds of "communities," which correspond to Tönnies' Gesellschaft (society), obviously differ from older communities based on blood bonds, at least because - as he puts it - its members "live peacefully alongside one another, but in this case without being essentially united - indeed, on the contrary, they are here essentially detached" (2001, 52). In other words, members of "arbitrary communities" are tied with much weaker bonds that become - as we have already demonstrated in Chapter Four - "cooled" through the process of reflexivity. Nevertheless, there is also something that makes communities of this kind similar to "natural" ones. After all, both create some form of collective representation, which becomes the source of social

382 We discuss both types of transformations at length in Chapters Six and Seven. 
identity. As a result, both allow recognizing a formerly "alien" individual as "one of us."

It is worth recalling here that insofar as natural communities rely on direct acquaintance or documented kinship as the basis for such recognition, in the case of arbitrary communities this is achieved, on the one hand, by the use of a formal proof of membership (e.g. party or union membership card, certificate of baptism etc.), and on the other - by forms of behavior whose main function is to create and sustain social distinctions (Elias 2000; Bourdieu 1979). The latter acquire special significance in the process of the transition to modern and contemporary societies, in which the weakening of social divisions and the blurring of the boundaries separating particular social categories make it easier for communities to be infiltrated by "aliens" - people whose presence would further endanger the already weakened bonds.

This leads to the second important process at the foundation of transformations of forms of sociality, namely the intensification of interpersonal communication. This process is certainly determined by technological progress - from the invention of the wheel and the domestication of draught animals to contemporary proliferation of electronic mass media. However, it is important to stress that the emergence of subsequent, ever more advanced means of communication is inseparably tied to both the above-mentioned social transformations, which extend the circle of potential interpersonal contacts, and to changes of social representations. In other words, new means of communication demand new kinds of users and new types of messages to be conveyed.

As far as the new users are concerned, we mean here not only changes in sensibility and ways of "decoding" messages, as discussed by McLuhan in his famous 1962 book The Gutenberg Galaxy, where they are treated as an outcome of the transition from oral communication to writing, print, and later to radio and contemporary electronic means. They certainly crucially affected the development of human intellect, as we indicate in Chapter Three. However, we also wish to point to those changes in the realm of praxis which homogenize the position of potential recipients, making them more susceptible to specific kinds of messages. The operation of such processes was indicated by Zygmunt Bauman, who emphasizes in his analysis of social conditions related to the rise of mass communication that it could emerge only in a society which embraced "universalization - supra-regional, supra-ecological and supra-class - of the essential components of the social situation" $(1972,65)$. $^{383}$

383 Among those "essential components" at the basis of mass communication, Bauman 
Although Bauman treats the phenomenon of universalization as characteristic of mass communication exclusively, it is worth underlining that it can be also discerned in basic modes of social communication developed in earlier historical periods of human societies, with one caveat, namely that the components determining social situation would be different. As we note in the previous chapter, what homogenized the situation of most people living in ancient societies was their dependence on the natural environment, which used to be a much bigger factor than it is today. The great "agrarian bureaucracies" constituted systems whose functioning and existing depended on rivers carrying fertile silts (Egypt), canals irrigating desert-like areas (Mesopotamia and Persia) and maintenance of elaborate irrigation systems (China). Along the same lines, it is possible to indicate that the factor homogenizing the social situation in the early Middle Ages was the specific "universalism" of recurring epidemics as well as of wars and robberies. The dissimilarity of factors homogenizing the social situation has particular significance for forms assumed by social representations we shall return to this soon. At this point, it is only necessary to note that at the basis of such homogenization we find the transformations of the sources of social strength as well as changes in the structure of power and wealth, which are consequences of the former.

The concept of social strength could be defined in more formal terms as referring to "the capability or opportunity to implement one's own will, to realize one's goals" (Giza-Poleszczuk 1991b, 86). It invariably constitutes a combination of individual characteristics (physical strength, intellectual capability, etc.) and resources available to individuals, which are derivatives of socially acknowledged forms of capital (Bourdieu 1986). ${ }^{384}$ Transformations of the sources of social strength understood in this way constitute a component of the third process that lies at the foundation of the evolution of human societies, namely the transformations of habitat. It is the kind of process that often inclines sociologists

enumerates: the dependence on the market, which we enter as ones who sell their skills or labor; the dependence on organizations that turn most people (regardless of their position) into officials of formal institutions; and finally, the dependence on technology, whose increasing complexity breeds disorientation and anxiety.

384 This concept is similar to that of social power, which appears in Elias's discussions, where he indicates that "the social power potential of a man in feudal society was exactly equal to the size and productivity of the land and the labor force he controlled," adding at the same time that "[i]n the feudal warrior society considerable strength was an indispensable element in social power" $(2000,234)$. Elias also emphasizes that social power "varies in its structure as much as does society itself" (234). 
to adopt the position of "technological determinism," which manifests itself in clear preference for those explanations that argue the development of tools (resulting from special properties of the human mind) to be a facilitator of social development which finds expression in the transition from traditional society to the postmodern one. However, the transformations of habitat can be also regarded from a different perspective as a process which, on the one hand, is conditioned by searching for new "ecological niches" for human collectivities, while on the other - by a system of social forces within those collectivities. In both cases, the existence of appropriate tools is separated from their use; therefore, the development of technology ceases to be the cause of social transformations. ${ }^{385}$

As we have already made clear, one of the factors that accompany periods of transition is the relative exhaustion of the habitat's resources, whose use serves as the basis for the expansion of a given form of social order. Such exhaustion can entail two fundamental consequences: either the society could face stagnation (or even decline), or its members could begin experimenting with new forms of behavior, typically turning to already existing patterns that have so far remained on the margin of social activity.

In those collectivities where the defenders of the status quo were for many reasons weakened, experimentation with new forms of behavior could lead to the transformation of that which constituted the individuals' main resource in their expansion in social space. The introduction of imitation processes on a large-scale would simultaneously entail changing the main factors that structure society. In this light, the transition from agrarian civilizations to industry- and information-based ones could be treated as a consequence of the search for subsequent "ecological niches" that would facilitate increase of social strength and expansion in social space, both for individuals and entire communities.

One could generally say that humankind has been transforming its habitat since adopting a settled lifestyle. ${ }^{386}$ The habitat, in turn, influences the changes of forms organizing social life through the consequences of our acting upon it. One

385 Naturally, this does not mean that it plays an insignificant role in these transformations. We only mean to underscore that accepting technological changes depends on social transformations and not other way round.

386 Obviously, it ought to be said that the process of transforming the habitat began not just when people adopted a settled lifestyle, but when they invented tools. However, as we have demonstrated in Chapter Two, the very fact of using tools is not specific to humans; moreover, those hunter-gatherer tribes that have survived to historical times were transforming their habitat to such a low degree that they were able to retain a relative "ecological" balance. 
of the most important consequences is population growth, which leads - on the one hand - to the intensification of communication, and on the other - to the exhaustion of those resources that are particularly intensely exploited within a given type of social order. This leads, in turn, to a search for new solutions, which also contributes to the upsetting of the social order.

\subsection{Transformations of the processes of socializing human actions}

The dynamics of social change described above allows to argue that the fundamental consequence of processes lying at the foundation of periods of transition from one type of social organization to another is the accumulation of such actions that not only fail to fit in the dominant form of sociality but also come into conflict with those patterns of interaction that this form sanctions. This causes the transition periods to abound in views expressing "world's rottenness" or "crisis" as new behavioral strategies violate the normative tissue binding the collectivity and, by the same token, undermine the principal "idea of society," which integrates the actions of its members.

This "idea of society" could be regarded as reminiscent of Kuhn's concept of "paradigm," which defines both the content of scientific problems and the ways of solving them. It was Kuhn who first drew attention - while accounting for the functioning of "normal science" - that it "does not aim at novelties of fact or theory" $(1996,52)$, but primarily strives to examine facts that are important from the perspective of currently prevailing theory, to confront them with that theory's statements, and to make more detailed findings. If scientific discoveries are made nonetheless, and new theories begin to be formulated, this happens as a result of a complex process in which various anomalies amass in the course of scientific praxis. Key stages of this process include the introduction of new terms and conceptual schemes, which is followed by the development of new theories and research procedures, ultimately giving rise to a new paradigm.

Following this analogy, one could say that the significance of processes discussed above - ones accompanying periods of transition from one form of social organization to another - is primarily related to the fact that they contribute to a particular increase of "anomalies," i.e. actions departing from that model of social praxis which establishes the identity of a given type of social order. This is further augmented by both the loosening of social control over individuals uprooted from their original communities as well as by the novelty of the situation in which they are forced to act, and by the intensification of communication with people subjected to different forms of sociality. The influence of 
such factors was underlined by Norbert Elias who emphasized - while analyzing the first phase of the development of the feudal system - that the settlement on conquered lands by warriors was accompanied by the loss of support provided by their original tribes. "Single families," he argues, became "ensconced in their estates and castles and often separated by long distances" (2000, 232). Similar consequences are carried by the migration from the countryside to cities. As Max Weber remarks, "[e]verywhere, $[\ldots]$ the city arose from a confluence and settling together of outsiders" (1978, 1237; emphasis added).

Let us note that although the emergence of various kinds of "anomalies" is a constant feature of social life, they are usually either incorporated into existing collective representations, or rejected, perhaps marginalized, so as to prevent them from distorting the prevailing image of reality. ${ }^{387}$ Thus, from an individual perspective, the essence of social life would be continuation rather than change. This is best illustrated by primal communities, which - as Lévi-Strauss notes - have a tremendous ability to reproduce themselves and may be thus "considered as systems where entropy is unknown or exists only in a very weak form" (Charbonnier 1969, 38). Even in contemporary societies characterized, as theoreticians of postmodernity unanimously claim, by an intensified pace of changes, individual lives may contain elements of dramatic turns or revolutions, but in most cases typically take a predictable course. The rhythm is set by daily routines, cycles of workdays and weekends, state and religious holidays, sales in shopping centers, flus in winter and vacations in summer as well as rites of passage (various initiation ceremonies, Communion, confirmation, Bar Mitzvah etc.) and weddings of grown-up children.

As is demonstrated in psychological research as well as in sociological or historical analyzes, even provided with easy access to the most sophisticated "peripheral tools" that are part of our cultural resources, the human mind is out of its nature - "lazy." It does not seek optimum solutions, but is satisfied with the first ones that offer an acceptable approximation. It does not strive to

387 David Riesman provides a good example of the first strategy by demonstrating that in primitive societies potential deviants were matched with certain institutional roles, e.g. those of a shaman or sorcerer. In his view, a similar role was performed by mediaeval monasteries $(1969,12)$. The second strategy is exemplified by the Church's campaign against usury in the Middle Ages. As Le Goff shows, the propagated representations of usurers would be meant to forge associations between unfairly earned money and oral or anal sex. Because the latter were separately condemned, the link was supposed to reinforce the negative assessment of the practice of lending money at an interest rate $(1990,34)$. 
grasp the "essence of things," but settles for a sense of obviousness. It puts new information in old forms, and when this is impossible, it rejects or ignores it. In everyday life, most people follow stereotypes, clichés and preformatted schemas of action, which are not individually derived from resources of human culture, but are copied from the repositories of social representations, assembled and maintained by the group (Moscovici 1981; 1983).

Periods of transition from one form of social organization to another upset this "laziness" not only by shattering the established order of things but also by confronting individuals with other representations of reality as well as new situations and problems, which require individual creativity to be solved. This causes behavioral "anomalies" to appear on a mass scale. Individuals must begin to behave in ways different from those that prevailed before not because they are driven to do so by some "inborn, human-specific" hunger for knowledge or curiosity about the world, but primarily because they wish to survive and use any opportunities to strengthen their position, adapting to the expectations of others. And since the most crucial feature of periods of transition from one form of social organization to another is the mass uprooting of individuals from their former communities, this entails freeing their experience from the cognitive frameworks constituted by community-produced collective representations.

One could thus say that the emergence of a new kind of social order is preceded by the shattering of the old, and a gradual construction of a new vision of reality embedding higher levels of interactions in lower ones and legitimizing higher-level products on lower levels. This is because, in periods of transition, interactions initiated by people as part of dyads or teams not only cease to be structured by images of reality sanctioned at the overarching level of the community but are also specifically curbed since - due to the dominance of direct regulations in collective life - individuals try to protect themselves from entering into undesirable contacts with others, especially unknown people, preferring to enclose themselves in more efficiently safeguarded small territorial communities created on the basis of various criteria. ${ }^{388}$ In order for these interactions to extend beyond the boundaries of those small "communities of experience," reality has to be interpreted in a new language, providing it with meaning and legitimization, which simultaneously turns certain types of behavior into anomalies or

388 This function was played in ancient cities by city walls. A similar role was taken in the Middle Ages by walls of castles, which offered refuge to people living in settlements outside the castle. Today, gated communities seem to have a similar task - ones protected by private security firms or at least by intercoms. 
"deviations," allowing for a marginalization of those people who exhibit such behaviors from the broader collectivity, or even their penalization. ${ }^{389}$

In other words, one inseparable element of periods of transition is the creation of a new form of "foundational holism."390 This calls for producing anew the kind of common knowledge that subordinates the striving of individuals to a superior whole, and designates a new basis for coordination of actions, now adapted to new forms of social strength created in the period of transition, as well as to new modes of communication and institutions of power. Obviously, processes of this kind do not take a smooth and peaceful course. As we have demonstrated in Chapter Four, images of reality produced within communities legitimize not only particular kinds of behavior undertaken as part of interactions among individuals but also the entire social reality, including the character of relations between individual communities within the larger collectivity, or - to employ classical terminology - the form assumed by the social structure and the places which individuals occupy within this structure. Their change therefore endangers the interests of existing communities, causing the periods of transition to be the kinds of historical moments during which we observe a particular intensification of ideological conflicts.

The content of these conflicts is to a large extent determined by the nature of processes that set the direction of changes in this fundamental vision of reality, which constituted foundation of processes of socializing human actions and had a religious character. According to Marcel Gauchet (1999), the basic function of every religion is to establish a specific relation between individuals, community and the environment. The essence of a religious vision of reality is the belief that the world in which human communities exist has been determined by an authority that is independent from them. This belief lends the religious view of reality the potential to stabilize the community. Indeed, it suppresses

389 Once again, we encounter here a significant analogy with transformations of scientific paradigms. As Kuhn observes, "discovering a new phenomenon is necessarily a complex event, one which involves recognizing both that something is and what it is" (1996, 55; emphasis preserved).

390 Naturally, in its basic form the act of "foundational holism" occurred only once in the history of human societies, although it was multiplied by its parallel occurrence in separate collectivities (we mean here the process analyzed in Chapter Two). All its other instances, its "renewals" or attempts to establish it anew in periods of transition from one form of sociality to another would involve participation of individuals who had already been somewhat socialized, and would occur in the course of struggle with individual habits as well as collective images of the world. 
reflexivity by imposing on people the view that the world order and the place they occupy within it are obvious. Finally, by foregrounding the belief that reality is completely determined, it thwarts any desire to change the world.

As Gauchet points out, the above characteristics of religious thinking were most fully realized in denominations that are unfairly called "primitive." ${ }^{391}$ They would conceive of the world as a unity that is fully determined by its mythical past. Neither people nor even any of the numerous semi-deities could challenge this kind of determinism. Man's religious dispossession of the possibility to shape reality "means becoming part of the living universe, being physically integrated into the heavenly cycles and into the supposedly permanent organization of the elements and the species. The potential antagonism embedded in humans' relation to nature is neutralized by substituting a symbolic attitude of belonging for one of confrontation" (1999, 26-27). The radical antagonism of individuals, inscribed in bonds that mutually tie them, is neutralized in a similar fashion. After all, "if the customary modes of human coexistence are accepted as wholly predefined, then no conflict arises between social actors about the content and forms of collective relations" (27).

The revolutionary change in this vision of reality is linked to the rise of monotheistic religions. They introduce the distinction between earthly, visible reality and divine, invisible one. As Gauchet claims, ascribing full power over reality to one, omnipotent God was the first step towards freeing people from the tyranny of pre-existing and unchanging conditions. After all, God would operate in the present and was not subject to any limitations established in the mythical past. Moreover, people could contact Him and negotiate. The separation of the eternal order from the earthly one, as well as the recognition of the fact that individual people can actually establish direct contact with God, who is responsible for the shape of the universe, made it possible to question the principle of community's complete dominance over all its individual members - the principle that was still in force in ancient Greece. This also caused people to note the fundamental difference between themselves and nature, thus opening the possibility of exploiting the latter.

However, before people regained, or rather gained the right to self-determination, which is the benefit of modernity, religious visions of the world still ordered

391 At the foundation of Gauchet's theory, we find the thesis that the great monotheistic religions, whose elaborate symbolism and complex organization have led many to regard them as representing a higher stage in the development of religion, were actually the first to pave the way for the process of secularization. 
and sanctioned human thoughts and actions, though on a different basis than it happened in primitive religions. The first outcome of the division of reality into incompatible orders - earthly and divine - was the attempt to degrade the former to the position of a second-grade component of life, one fully subordinated to the religious order. As Gauchet notes, since Israelites were a society that fell under the domination of a foreign power, they were the first to call into existence a God who would not just disobey earthly authorities, but clearly oppose them. It needs to be remembered, however, that Judaism established a clearly defined boundary of its earthly community as an association based on both faith and origin (Morris 1996) - in this sense, it has remained a quasi-tribal religion despite its opposition to secular authorities. ${ }^{392}$

Christianity went much further in establishing the division between earthly and divine orders. As discussed in the previous chapter, in the first period of its development Christianity would "extract" individuals from the earthly order, subjecting their actions to a strictly religious one. In the period of decadence of the Roman Empire, Christianity acquired the status of state religion, which stopped this process, assigning to the Church the role of mediator ${ }^{393}$ between the material world subjected to secular power and the invisible world subjected to the will of God. At the same time, however, it supported the limiting of the monopoly enjoyed by authorities ruling the earthly world, thus establishing a source of potential conflict between two organizations - secular and religious each of which would aspire, with the passage of time, to rule over the entire Christian world.

What seems even more important, after the failure to rebuild the Roman Empire, and a long period of fragmentation of secular organization, the mediaeval Church became the only universal authority that not only created a general

392 The later Jewish diaspora had to consolidate this communal character of social organization because it precluded the emergence of its own political order separate from the social one.

393 Let us note here that it was Christianity, or more precisely Protestantism, that questioned the necessity of the existence of any such mediating institution (this is one of the reasons why Gauchet considers Christianity to be the first step towards secularization). If - as Christian doctrine would have it - God does not occupy the highest place in earthly hierarchy, but remains outside it, the existence of an earthly instance mediating between God and people seems dubious. Nevertheless, the Church would successfully defend its mediating position for many centuries (until it was openly attacked during the Reformation), presenting itself as Christ's heir and thus as an institution that is not entirely of this world. 
vision of the world and integrated a supra-state community of believers but also developed models of life in accordance with the universal, i.e. divine order of things. However, these models did not have the same kind of power to regulate human actions as in primitive religions. One could, after all, negotiate with God - and especially with the Church that represents Him on earth - with regard to such matters as the absolution of sins and obtaining forgiveness (by repenting in a proper way) for actions that would stand in complete opposition to the dominant vision of the world.

The increase of the significance of secular power, which began in the fifteenth century, was not achieved by extending the area over which it had control ${ }^{394}$ but by tightening its grip on the current territory, i.e. by consolidating power over the actions of all members of state organization. The sovereignty of the ruler in relation to the Church began to be tied to representing ("personifying") the community of subjects and satisfying its needs, e.g. by guaranteeing safety. According to Gauchet, it was this secular concept of sovereignty (fully developed in absolutist monarchies) that became the foundation for the rise of modern democracy. The transition to this form of political organization demanded, Gauchet argues, that the ruler, as guarantor of the community's existence, be replaced by impersonal concepts of "nation" and "state." Both ideas transcend their particular realizations and, in this sense, do not belong to the order of the material world, at the same time remaining independent from any power positioned outside the human community. This means that they also do not belong to that order of the world which is subordinated to God.

However, it is easy to demonstrate - as we have done in the previous chapter that whereas the concept of the nation really established a new kind of community, the idea of the democratic state rather involves a group of specific institutions performing their tasks in the name of that community. Their development was preceded by a period of struggle with the absolutist state, which Gauchet does not address. ${ }^{395}$ This battle was fought under the banners of civil society - a concept opposing both the aspirations of the Church, and the striving

394 It can be argued, however, that the establishing of the Anglican Church was an attempt at incorporating religion into the sphere subjected to secular authority.

395 Gauchet's silence about the significance of liberal and republican civic movements may stem from the fact that his argumentation - as the very title of his book demonstrates is subordinated to displaying mechanisms that lie at the foundation of secularization processes. That is why he concentrates primarily on the sole fact of the separation of earthly power from its divine counterpart, without discussing historical details that conditioned the transformations of the former. 
of rulers to gain control over the earthly lives of their subjects. ${ }^{396}$ The concept of civil society actually vanished from public discourse in the nineteenth century, but this was because the battle was already won and former subjects became citizens of a modern state which exercised government in their name. At the same time, they would become members of a single nation, or at least attempts would be made to impose such an identity on them.

It was the state that "used the concept of nation for its own purposes - i.e. to advance its power and prestige - and greatly obscured this idea, which was still rather clear in the eighteenth century" (Żelazny 2004, 69; cf. also Anderson 2006). Initially, the term would refer to the common origin of an ethnic group distinguished on the basis of using the same language. States, whose creation and territories depended on a tangle of complex historical factors, would encompass within their territories many "nations" understood in this way, i.e. as ethnic groups. Ultimately, it was not the "will of peoples" but the effort of politicians that "has led to the equation of state and nation in an attempt to convince everyone that states are created by nations, not the other way round, and that there are no other entities of this kind on earth" (73). In this way, the ideology of nation as a socio-political community carried inside it - from the very beginning - an inherent component that would dismantle it from within during the twentieth century since it also brought to life the concept of national minority.

The nineteenth century and the first half of the twentieth were a period of intense efforts undertaken in European and North American countries in order to create a single community out of many, more or less dissimilar ethnic components. ${ }^{397}$ These also included other dissimilarities within the community such as distinct interests stemming from social differentiation. The new entity would be guided in its actions by the kind of a vision of the world in which the interest of the state constitutes the common good, with regulations introduced by this body offering the basis for individual and collective actions. This goal was achieved in many countries. From the middle of the twentieth century, however, it began to be clear that this blending process was rather superficial. The national community began to crumble not only under a pressure from its

396 The development of the concept of civil society in England and France occurred parallel not only to the strengthening of the "third estate" but also, first, to processes of breaking the power of aristocratic families, and second, to the monopolization of power by monarchs.

397 The meaning of these efforts is best reflected by the term "melting pot," used with regard to the USA, which creates a vision of various base ingredients fusing so as to create a single homogenized "product." 
increasingly multi-cultural character augmented by intense migrations, or the suddenly awakened national consciousness of groups that formerly seemed to be integrated within the political and cultural framework of the nation state, but also because of various kinds of cultural, sexual and physical differences that people began using to gain a minority status within the society (e.g. women, homosexuals, disabled people etc.). The place of national identity, acquired by the very fact of being born in a particular state, often began to be replaced by social identity adopted through individual choice.

As Claus Offe shows, towards the end of the twentieth century "the politics of identity-based differences is an increasingly prominent feature of increasing segments of the contemporary world, developed and developing alike" (1998, 122). It has also become increasingly common to use symbols of social identity in an instrumental way after they gained more significance due to the basic properties of the democratic political order. Offe draws attention to the fact that in Western constitutional democracies social conflicts would be often alleviated by granting special group rights to certain unjustly oppressed structural minorities. In contemporary Western societies, the instrumental use of social identity is fostered by the fact that the scope of the term "oppressed minority" is easily blurred. A large majority of the society, basically "everyone but relatively welloff, relatively young, able-bodied, heterosexual white male" - as Kymlicka writes $(1995,145)$ - can convincingly claim to represent some "oppressed minority" or consider themselves part of it in good faith.

The battle for equal rights, fought under the banners of social justice, becomes in this context not only a symptom of the crisis of nation state as a political and cultural community but also a testimony to the rejection of that mode of organizing social order which was characteristic for the modern society. Paradoxically, however, it also leads to what Gauchet has called elsewhere "the complete triumph of the state which enables the manifest triumph of the liberal individual" $(2000,32)$. As he demonstrates, the collapse of that principle of integration which lies at the base of the collective "We" characteristic of the modern society is related to processes of the state capturing the "social bonds" created in various communities, as we signaled in the last three chapters. ${ }^{398}$ Along with the "privatization of the family" - as Gauchet observes, arguing that the family has become primarily a psychological relation, thus ceasing to perform the function

398 Gauchet writes about "the appropriation of the social bond by the state; we might even say that the modern state is born in and through this appropriation, and that the latter serves to establish the very concept of the state" $(2000,29)$. 
of a social institution - "we are witnessing the final phase of the concentration of the social bond in the state" (29). One effect of such "nationalization" of social bonds is the spread of the belief that these bonds exist solely by the fact that we are Poles, Frenchmen or Americans, i.e. representatives of the same nation. "We are the inheritors;" Gauchet writes, "we enter an already constituted world which includes not only unsurpassable canons in thought and in art but equally is ordered around preregulated forms of coexistence with others" (33).

"The implicit production of the social bond by the state," Gauchet concludes, "means that the explicit social bond is experienced only as a global effect of the aggregation of actions where each person considers only his own advantages and interests, with the outcome that the social bond appears as a result and not a responsibility" (32; emphasis preserved). The dissolution of bonds, documented in many studies, appears therefore as a consequence of an overly optimistic belief in the power of national bonds. Just like any other kind of bonds, they are nevertheless doomed to lose significance because, along with the psychologization of the family, individuals have ceased to be subjected to the kind of socializing training that would be linked to the creation of a moral universe. ${ }^{399}$ Therefore, " $\mathrm{t}$ ] he fundamental characteristic of the contemporary personality" would consist in the fact of today's average person being "unaware that the basis of his being is not organized by the precedence of the social and by embeddedness within a collectivity, with all that this implied for thousands of years regarding the feeling of obligation and the sense of debt" (36). Most activities have been subordinated to individual desires and preferences, which are limited only by the efficiency of law. ${ }^{400}$

Thus, the only factor that allows people to share actions and visions of the world - at least in the Western sphere of influence - is now the knowledge of experts, to which individuals resort both when they want to "manage" their

399 The overtaking of the function of "the family as social institution" by the state was already emphasized by Ulrich Beck (1992), who regarded it as one of the key reasons for the structural individualization observable in contemporary society. Karin KnorrCetina (1997), in turn, draws attention to the fact that in contemporary Western society individuals cease to be socialized to fit the community and are increasingly often socialized to fit objects, "cooperating" with which becomes all the more important since they more and more frequently replace people in relations that are by definition of social character (e.g. online banking, helplines where one can obtain information about a given institution by telephone).

400 An extreme example of this is the question of lawsuits filed against parents by children in USA or Sweden. 


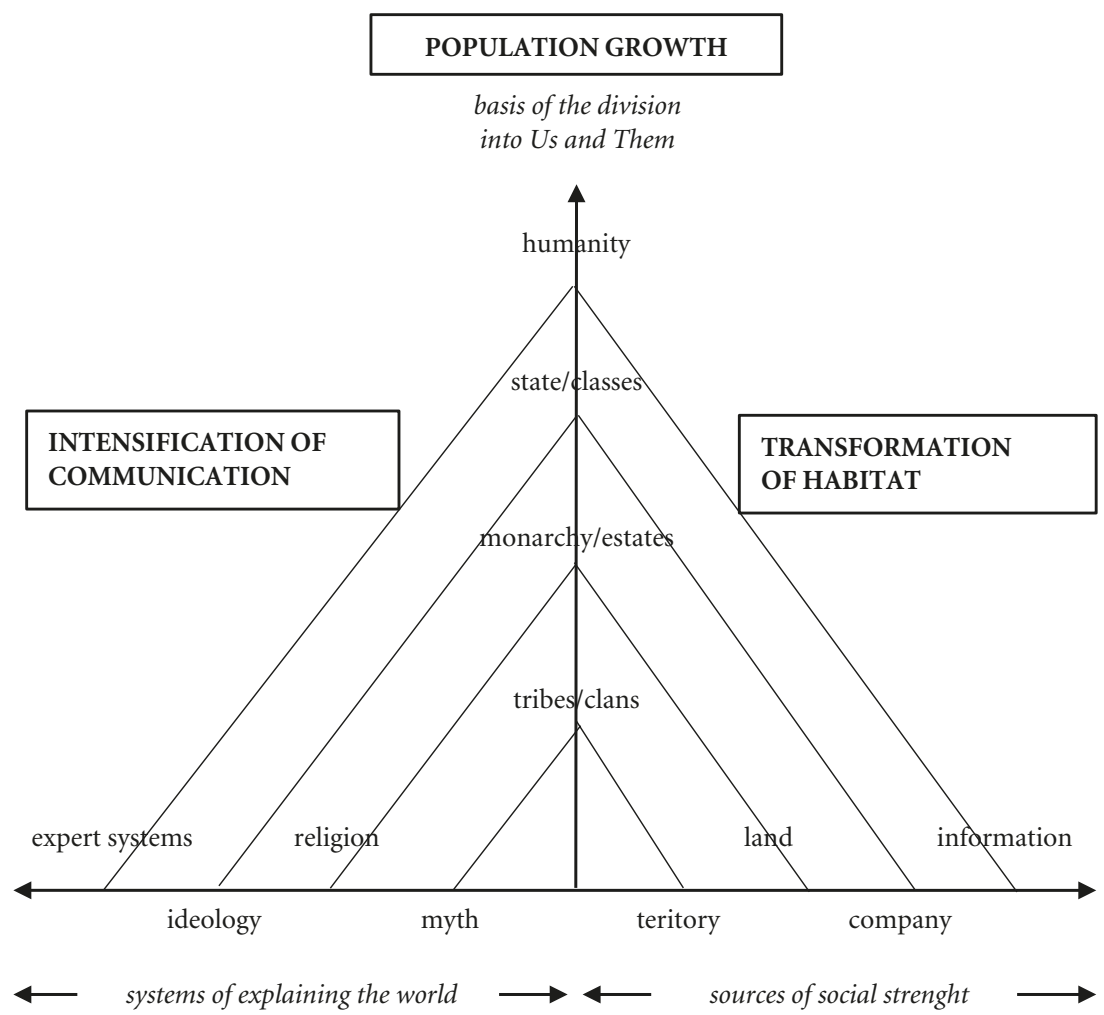

Fig. 8.1: Summary of changes occurring as part of "systemic transformations"

strivings more efficiently, and when they attempt to maximize the usefulness of social institutions and other people while trying to achieve their own goals. Strictly speaking, since that knowledge is also differentiated, the contemporary society offers its members various expert systems. It is by choosing from among them that individuals come to share their lifestyles and visions of reality. However, the development of such "internally referential systems," which lie at the foundation of the "reflexive project of the self" (Giddens 2006, 231), is not supported by bonds existing between individuals, nor does it impose any moral obligations on them. In other words, it does not create a community.

A summary of relations between mechanisms and "products" of processes found at the foundation of transitions from one form of sociality to another is provided in fig. 8.1. 
This figure requires two commentaries.

First, in case of all historically identified transitions from one form of social organization to another we deal with gradual processes of change that precede them. Such events as the "Hun invasion" (which was not a one-time event, but a prolonged movement of tribes) or "information revolution" constitute merely a sort of catalyst or "attractor" for these changes, which facilitates the creation of new "repeated assemblies." After all, from the perspective of Braudel's longue durée approach to history, processes of social life ought to be described in terms of periods of "transition," extended in time and punctuated with short episodes when given types of social order would be stabilized, reaching their model form. In this sense, it turns out that the diagnosis about the processual character of social life, formulated by the theorists discussed in Chapter One, is in fact right. Processes modifying social orders occur in parallel to their crystallization. ${ }^{401}$

At the same time, it is not hard to see that periods of stabilization or stagnation occurring in human history are related to the gaining of relative advantage by one of the factors in which all three basic mechanisms of change are rooted. It could be - as in the case of China - the existence of a strong social group capable of curbing the spread of unlegitimized actions. It could be - as in the case of those hunter-gatherer societies that have survived to this day - an unfavorable, poor habitat that does not offer many possibilities of experimenting with the environment. Finally, it could be - as in the case of ancient Egypt - an unusually coherent image of reality based on the cult of ancestors, one that regulates the behavior of individuals and relations between groups in a particularly detailed manner. In all other cases, the mechanism of change functioned almost automatically because it would be related to processes of interdependence set in motion by a series of positive feedback reactions.

Despite the fundamentally processual character of social life, the fact that sociology has adopted the habit of analyzing processes of change in terms of transitions from one form of social organization to another does seem to be justified, at least due to two different circumstances. The first is related to the fact that both types of social order identified so far (whose existence is universally acknowledged in social sciences) - i.e. the traditional order and the modern

401 Durkheim and Marx's descriptions of nineteenth-century society as formless and full of anomie (quoted above) concern that stage in the development of the modern order which we consider today as classical. However, we can see now that this stage contained all the elements that have later led to the transition creating the postmodern order. One could thus say that, paradoxically, a given type of social order achieves its clearest form at the moment preceding its fundamental change. 
one - are clearly characterized by different modes of organizing collective actions at the four configuration levels of interaction distinguished earlier (cf. Chapter 4): modes that are relatively stable in longer stretches of time. The shattering of the previous mode of social organization, which occurs during a transition period, and the process of replacing it with a new one is thus the second factor justifying the analysis of social change in terms of basically distinct types of social order following each other.

Second, it is necessary to address the question of the place occupied by the postmodern order in the proposed outline of the evolution of fundamental forms organizing social life. The changes in systems of explaining the world as well as shifts in sources of social power and transformations of the "Us/Them" divisions (see fig. 8.1.) suggest that we consider the postmodern order as distinct from others. At the same time, however, earlier accounts of processes leading to the change of the dominant form of sociality would suggest that what we have come to call postmodernity displays all features that are characteristic to periods of transition or dissolution rather than to some new, already crystallized form organizing social life. Therefore, we need to finally confront the question posed at the beginning of this book - one about the status of currently observable changes - and to clearly answer whether they indicate the emergence of a new type of social organization. 


\title{
CHAPTER NINE. TOWARDS THE POSTMODERN SOCIETY
}

\author{
A society made up of an infinite scattering of disparate \\ individuals, which an overgrown state attempts to \\ limit and restrain, constitutes a veritable sociological \\ monstrosity.
}

ÉMILE DURKHEIM, The Division of Labor in Society

\begin{abstract}
The past cannot explain or predict the future, but it can set very definite limits on change and direct its general direction.

DANIEL CHIROT, Causes and Consequences of Backwardness
\end{abstract}

Social structures are always structures of social norms

RALPH DAHRENDORF, Life Chances

In the mid-1980s, one of the prominent Polish social scientists summarized his impressions of the USA in the following way: "Crisis. Economic crisis. Social crisis. Crisis of education. Crisis of family. Crisis of everything. Crisis is giving sleepless nights to the unemployed and the soon-to-be-unemployed. Businessmen, managers and politicians" (Osiatyński 1986, 7). American sociological writings of the 1980s and the 1990s document this crisis in various domains of social life. These include celebrated books by Allan Bloom (The Closing of American Mind, 1987), Richard Sennett (The Fall of Public Man, 1974; Corrosion of Character, 1998), and Robert D. Putnam ("Bowling Alone," 1995).

This period also saw the publishing of studies whose authors do not limit themselves to registering symptoms of crisis but seek to identify deeper processes transforming modern societies. In the preface to his seminal work, ${ }^{402}$ Ulrich Beck declares that his goal was "to move the future which is just beginning

402 Within years of Beck's book (1992), Zygmunt Bauman published Legislators and Interpreters (1987), while Anthony Giddens - Modernity and Self-Identity (1991). Both strongly emphasize the claim that modernity has "exhausted itself" and entirely new forms of social action emerge. The three authors have thus determined a new framework for reflection on contemporary society for many years to come. 
to take shape into view against the still predominant past" (1992, 9; emphasis preserved). He formulates a strong thesis: the modern industrial society has begun to transform into an individualized risk society, in which the distribution of risks replaces the distribution of goods. Beck's concept of risk society gained immediate popularity as it corresponded to the subjectively experienced crisis, aptly rendering the unique sense of helplessness in the face of entangled processes that began to shape social reality. At the same time, it occluded another, theoretically more promising concept developed by Beck, namely that of structural individualization, which accounts for the consequences of the dissolution of modern social structures.

Later, structural individualization was simply replaced by individualization conceived as the fundamental feature of contemporary society. As we demonstrate in Chapter One, this concept has dominated sociological reflection on ongoing civilizational transformations, reinforcing sociology's individualistic "bias" and consolidating its division into two unrelated variants: one analyzing "individualized society" and the other focusing on changes in social structures by referring to its own concepts that do not take into account processes of individualization. ${ }^{403}$ In our view, an analysis of social transformation in terms of changing forms of sociality - proposed in this book - could help close the gap between these two sociologies.

\subsection{From a society of local communities to a discreet society}

As we set out to show in Chapter One, the image of social life produced by sociology includes the conviction that society is basically identical with the state, the latter being - like other institutions and organizations of the "lower order" - the product of society's "collective will," its representation and simultaneously executive apparatus, and that social life involves individuals forming larger groups formal or informal - which negotiate among themselves the directions of social development and goals meant to be realized by the state. All such convictions are based on analyzes of events accompanying the only historical example of radical change in social order: the transition to modernity. According to Jacob Bronowski, among these events a significant role was played by the British Industrial Revolution, forming a specific triad with the American and French Revolutions. Although he would argue that "[i]t may seems strange to put into

403 A major role seems to be played in them by the concept of civil society and social capital, though the concept of social class would be still used. 
the same packet an industrial revolution and two political revolutions," such an operation would be entirely justified because "the fact is that they were all social revolutions" (2011, 2513-2521; emphasis added). One could also add that all of them were the work of individuals attempting to overcome limitations imposed on them by the traditional form of sociality. However, it is important to remember that although this coincidence led to the overlapping of changes in social, economic and cognitive orders, each of these transformations was distinct and could be analyzed separately.

Therefore, if we restrict ourselves to the social dimension, what ought to be drawn from the above "packet"? This question is all the more pertinent because since the time of the three revolutions enumerated by Bronowski it has been also filled with new revolutionary events and processes: the second industrial revolution swept through the world, demographers speak of "the second demographical transition," while means of communication have been also revolutionized. All of this is somehow linked to changes in forms of sociality, but the habit of defining sociology by indicating its distinct object of study makes it difficult to identify these links. Focusing on the object favors treating specific characteristics of the modern form of sociality - such as ones related to its becoming "society" and creating the concept of "nation state" in order to sustain the integration and coordination of actions - as universal correlates of socializing processes. ${ }^{404}$ As long as we keep reifying these processes by identifying them with society understood as a separate "object" of study, we shall remain unable to analyze their transformations in a properly sociological manner.

What granted sociology the status of a distinct scientific discipline was its concentration on the analyzes of changing forms of sociality, i.e. people's tendency to live together in various types of communities. Therefore, instead of deliberating about the miserable condition of society - which is characteristic for today's sociology - we propose to focus on the analysis of socializing processes, understood here as processes that turn individual behavior into the streams of coordinated collective actions. Such a change of conceptual framework makes it possible, on the one hand, to reach the level on which sociology's basic domain of interest is situated, and - on the other - to avoid pitfalls related to the inevitable routinization of all human actions, which naturally include sociological research practices characterized by the tendency to treat society as an entity that emerges

404 The logical consequence of identifying socializing processes with a historically specific "society" is to view the currently experienced transformations of dominant forms of sociality in terms of society's dissolution. 
from the distribution of answers to survey questions. For obvious reasons, the concept of socializing processes cannot be reified. At the same time, it is basically irreducible, which means that it can be identified at the foundation of all kinds of structures emerging as a result of human actions.

In their daily course, socializing processes involve creation (cooperation), re-creation (socialization), and sustenance of social bonds (through rituals). These bonds can have a more "natural" or "arbitrary" character depending on the extent to which reflexivity partakes in initiating and shaping cooperation. It is possible, therefore, to assume that the share of arbitrary bonds increases along with the intensification of those changes in "cultural devices" which condition (in the historical dimension) the transformations of ways in which the human mind functions. The strength of bonds depends on the strength of emotional bonding formed between individuals and the group. This, in turn, is conditioned primarily by the course of socialization processes, which can either curb or enhance the ability to enter such relationships. We shall stress once again that emotional bonding is not synonymous with experiencing positive feelings. Its constitutive component is having an affective relation to the entire community so that this community becomes - regardless of specific feelings experienced in relation to it - an important framework for individuals.

Communities are distinguished on the basis of their specific tasks (coordinating actions) and the functions (creating common knowledge) they fulfill in organizing social interactions, not on the basis of their more particular properties. In this sense, a community could be constituted by a "primary group," by a formal one comprised of people who are its members only in some aspect of their activities, or by a larger group of individuals who have a chance to communicate with each other, share a certain image of reality, and cooperate in order to achieve certain joint goals. Whether any specific group could be called a community is determined by empirical criteria, not ones related to classification. Today, an individual can belong to many communities, especially because many of them have an aspectual character.

The constitutive component of each community is cooperation and sharing a common image of reality. It is in the process of cooperation that bonds develop among individuals and common knowledge about reality is agreed upon, in consequence of which certain elements of that reality (primarily the concept of the common good and a sense of the collective "We") begin to be considered as obvious and indisputable, in turn becoming a framework for the coordination of individual tasks and integration. It is also in the course of cooperation that mechanisms of social control are initiated - appealing to shame, guilt or pride 
(Shott 1979) - at the basis of which we find emotional bonding of individuals through the community.

The coordination of actions and integration of community members necessitate creating a public sphere within each community. It serves as an area in which common knowledge is identified and sustained. In the historical dimension, these two tasks are also the beginning of two first public institutions whose emergence accompanied population growth. They would be formed by people specialized in "managing" the community (chieftains and their "administration") and people specialized in "managing" common knowledge (priests). Consequently, institutions can be considered as a formalized modus operandi rooted in tasks faced by the community. Their shape and structure say a lot about the modes in which a given collectivity functions, but it needs to be kept in mind that all institutions (including those of the state) should not be considered as independent entities, but - as the sociological point of view suggests - as a means of delegating solutions to certain typical problems to specific groups, and consequently as areas where "aspectual" communities form. They also strive to survive and thus generate their own tasks and common knowledge.

Communities do not exist in isolation but they establish contacts with others in order to facilitate exchanges and share language. It is at the level of macrodeme that the public sphere sensu stricte merges, providing a space for interactions between people who did not know each other before but who accept certain rules of coexistence. The existence of such rules facilitates the emergence of three abstract "macro institutions": the market, state and communication system. They ought to be treated as distinct domains within the public sphere, gathering specific types of interactions and simultaneously generating forms of order specific to them. Whereas the communal public sphere is governed by tradition and mores, in the public sphere in the strict sense, norms of coexistence begin to be ordered by formalized rules that sometimes take the shape of law.

To account for transformations of socializing processes in our theoretical model, it becomes necessary to include in it the historical consequences entailed by the three basic mechanisms we have identified as stimulating changes of human communities: population growth, intensification of communication and transformation of habitat (cf. fig. 8.1.). In the broadest terms, these consequences could be reduced to transformations of basic "units of survival" (cf. Chapter Three) in which human communities function. If we slightly modify the division proposed by Norbert Elias, one could say that these units of survival embrace local communities (including tribes), states and humanity. This division does not refer to bonds or forms of sociality, but to objectivized, historically created conditions of human existence, characterized by an increasing interdependence 
of territorial units occupied by particular collectivities, and consequently by the increasing probability that events occurring at one point in the organization may affect all others.

It is the transformation of basic units of survival that gives rise to "imagined communities" - the kinds of the collective "We" that refer primarily to territorial communities: "We, the Varsovians," "We, the Catalonians," "We, the Poles," and "We, human beings." ${ }^{05}$ As Benedict Anderson notes while discussing nation as an imagined community, "members of even the smallest nation will never know most of their fellow-members, meet them, or even hear of them, yet in the minds of each lives the image of their communion" $(2006,6)$. Preserving the "image of their communion" as well as developing an emotional bond with such a community are processes that naturally take place at the level of actual communities (especially the family), for which the sustenance of this kind of interdependence constitutes the necessary condition for effectively defending their own territory - a part of the basic unit of survival. The development of territorial imagined communities would be thus conditioned primarily by transformations of means of communication: both those related to spatial mobility and those related to interpersonal contacts.

Let us emphasize here that adopting such a perspective makes it possible to distinguish another type of imagined communities apart from territorial ones, namely "ideological" communities. We understand the term "ideology" in the way proposed by John B. Thompson, who argues that it "can be used to refer to the ways in which meaning serves, in particular circumstances, to establish and sustain relations of power which are systematically asymmetrical - what I shall call 'relations of domination'" (2013). In other words, ideological imagined communities emerge as a result of objective social differentiation, which is rooted in transformations of sources of social strength; thus, they may serve not only to sustain power relations but also to modify them.

The first such imagined ideological communities were formed - in line with the findings of Marcel Gauchet, discussed in the previous chapter - by monotheistic religions. ${ }^{406}$ During the Middle Ages, the Church would not only legitimize

405 The primal form of such a territorial sense of the "We" seems to manifest itself in the answer "I'm a local" to the question "Where are you from?"

406 Both Judaism and Christianity were born as religions questioning existing relations of dominance. In the former case, this was political domination, while in the latter - at least initially - social domination. 
existing power relations, sanctifying the reign of particular monarchs $s^{407}$ and providing religious justification to established social divisions, but also mobilize a supra-state Christian community to act in such a way as to change power relations in areas beyond its sphere of influence (through missionary activities or crusades). In the modern period, in turn, the consolidating community of affluent groups would develop not only the concept of "civil society" - one that undermines political authority existing at that time - but also ideas supposed to legitimize emerging social divisions, accentuating that the path to affluence is open to anyone willing to work hard (Anthony 1977). The slightly later international workers' movements created an alternative imagined community whose aim was to put an end to unfair social relations. Nowadays, the crucial forms of such ideological imagined communities include feminism and all other variants of minority movements striving to change existing relations of dominance and subordination.

Just like territorial communities, ideological ones are also created and sustained within real communities (of work, organization, etc.). We can consider both as communities only because of the fact that they create a certain kind of common knowledge $\mathrm{e}^{408}$ and the kind of bond that imposes moral obligations on people with respect to the community they identify with. ${ }^{409}$

407 In certain cases, and historical periods, the Church would do the opposite: by delegitimizing secular authorities, it would attempt to change existing relations of dominance and subordination.

408 Its crucial component is constituted by various kinds of "founding myths" in case of territorial communities and "myths of enslavement" or "myths of merit" in case of ideological communities questioning or legitimizing existing social order. The former term is not intended to suggest that there are no authentic, often deep and unjustified social inequalities. We merely wish to emphasize that, in the process of creating ideological imagined communities, they are invariably mythologized. The same is true for "myths of merit." One could assume that the fundamental principle underlying contemporary differentiation is one of meritocracy, but it cannot be argued that its operation in contemporary societies is failsafe, or that people who have secured higher positions are more intelligent, knowledgeable or competent in comparison with those who have not made such careers. This would be the essence of the "symbolic violence" analyzed by Pierre Bourdieu (cf. Bourdieu \& Passeron 2000).

409 The division into territorial and ideological communities (assumed here) to some degree coincides with the model developed by Paul Morris (1996) who distinguishes communities of descent and ones of assent. Membership in the former is defined by objective criteria, usually ones like place of birth or indicators of social status, and is given to the individual on the basis of social conventions. Membership in the latter, 


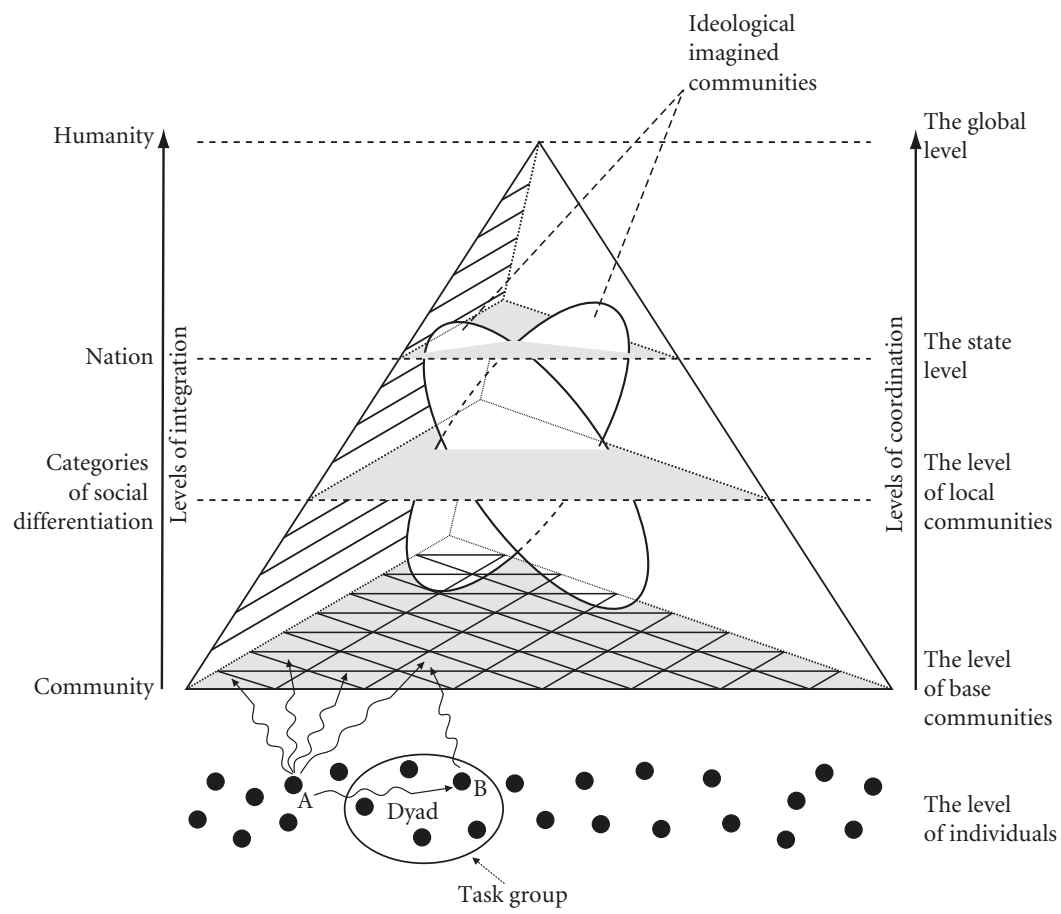

Fig. 9.1: Levels of coordination and integration in contemporary society

One could therefore say that along with the increase in the complexity of human societies, the tasks of coordinating actions and integrating individuals through common knowledge, initially realized by base communities, were not replaced but supplemented by creating higher levels of coordination and integration, which - through the development of imagined communities would refer to the same socializing mechanisms as those used on the base level. The concept of society emerges, in this configuration, as yet another kind of "imagined community" - in this case, one conceived by sociologists who attempt to synthesize in one term the entirety of various relations and social affiliations.

A summary of our findings is presented in fig. 9.1. At the same time, it offers a schematic account of processes of changes in basic forms of sociality, rendered

on the other hand, is a matter of individual choice. Cf. also Morris's interesting discussion of contemporary changes in these two forms of communal bonds. 
in terms of the transition from traditional to modern and contemporary society, the last one formed in an already globalized world. These forms differ in many crucial aspects, but we shall limit ourselves here to distinguishing dissimilarities in the basic principles of organizing social order.

Traditional society was - and still is in the regions where it survives - a federation of relatively isolated small local communities that embrace entire lives of their members. Integration of this federation - in itself problematic and tentative - would rely on the loyalty of community leaders to the center, and on their members' belief in the image of the world created by religion. Within this configuration, individual sense of belonging would take as its object an unspecified spiritual community rather than any particular system, such as the state.

In Europe, this society of local communities began to crumble in the middle of the second millennium CE. Along with urbanization and industrialization (even proto-industrialization), individuals were "uprooted" from their "small homelands" and located in an anonymous urban space deprived of communal areas. One could therefore say that the growth of cities and the rise of an anonymous crowd formed the first image of mass society: an anonymous assembly of uprooted and unrelated people, deprived of mechanisms of integrating and ordering their actions. However, certain structures of organizing and coordinating actions did begin to crystallize and grow out of this chaos. Various aspects of these actions were separated, fragmented and subordinated to the rationale of abstract institutions and mechanisms.

The society that emerged in the course of such long yet endogenic processes could be called the society of large structures. Economic activities (including work) began to be organized by the market, and mighty nation states would be born along with their administrations, which were organized in accordance to the idealized principles of bureaucracy described by Weber. A robust industry based on mass production emerged, its symbol being the assembly line - the embodiment of rational division of labor and distribution of tasks. A new political "arena" was formed along with principles of democracy and political parties. Mass culture arose, as well as universal education, legal and court systems and so forth. Masses of people began to "organize" themselves as social classes, both literally (as trade unions) and in the form of distinctive subcultures and lifestyles.

The emergence and functioning of these large structures would be impossible without a specific system of coordination created by the modern nation state. Although this system could not develop without appropriate individual motivations, it could set itself free from them as soon as it was born. ${ }^{410}$ To put it

410 Motives do not characterize the market but individuals. Markets or bureaucracies are not the goals or values of individuals. 
differently, individual intentions have little significance in a properly rationalized system in which rationality of action is reserved for structural positions, not for individuals. Coordination of individual actions in modern states could be envisioned as an abstract and well-described organizational structure that individuals enter after meeting certain requirements and simply becoming executors of actions ascribed to particular positions.

Getting rid of engaging in the functioning of structures that are "making it on their own" (i.e. rational in themselves, whereas individuals are exchangeable - it suffices that they fit a given position), people freed themselves also from social bonds. This entailed the rise of masses of "loose" individuals who would not be connected to each other with any ties. Deprived of moral obligations to the community, they have to seek their identity and meaning of life elsewhere. This, in turn, leads to the intensification of a sense of non-authenticity and enslavement: after the ethical element was eliminated from large structures, work loses its religious dimension; it is no longer a vocation and becomes a mere way of earning money. The rebellious "mass-man" of Ortega y Gasset (1994), alienated from the civilization that determined his or her reason for existing, ultimately turns against it.

As a result of this, in the middle of the twentieth century processes of "implosion of large structures" have begun. They are evidenced, on the one hand, by counterculture, and on the other - by the spectacular economic success of small firms and the fall of some gigantic companies. However, the implosion of large structures did not lead to their disappearance but rather changed the way they function, making them invisible. This change would consist in transferring control from the sphere of internal structures to that of boundary conditions. In other words, organizations (structures) abandon strict control of internal principles of operation ${ }^{411}$ in favor of control over their general parameters, especially external ones, to which they are supposed to adapt flexibly. A new fashion is thus born - one for a "soft" structure.

The "implosion of structures" has also involved class structures. ${ }^{412}$ The new "middle class" emerged - one embracing most of society and deprived of a homogenous, bourgeois character. It is impossible to characterize this class using criteria such as affluence, profession or education because it would include

411 We mean here principles that manifest themselves in clear hierarchies of power, distributions of positions, regulations, etc., which are often identified as sources of inefficiency in organizations and the origin of "vicious circles" in bureaucracies.

412 In sociology, this is accompanied by a departure from the concept of "class" in favor of "social stratification." The latter signifies a softening of the criteria of social divisions. 
professionals, small-time proprietors and wealthier workers. The upper classes include both high-level managers and large-scale proprietors. The lower class begins to be formed - to an increasing degree - by immigrants. A discreet society is born.

The term "discreet" refers to several key aspects in the contemporary organization of social life, ones often discussed in theoretical accounts of the postmodern society. Among them we find: the invisibility of structures, which increasingly often assume the shape of processes initiated by slight changes in parameters and controlled by small groups (for instance, decisions about discount rates taken by managements of large financial institutions); their fluidity (global mechanisms do not form any clear organization despite constituting elements of processes that affect almost everyone); "discontinuity" (since structures take the form of process, they break into a series of "discreet" units); and finally, pluralism (right next to the giants, various small structures would appear: NGOs, small political parties, firms and local communities). The rise and popularization of the internet has intensified communication, invariably leading to what Castells (2001a) has called the "globalization of flows" of all values that can be expressed electronically. Capital as well as contemporary businesses and corporations cease to be tied to a particular place, while the time needed to communicate has shrunk to a "permanent present."

The "globalization of flows" also includes transformations of relations formed among people. After all, structural individualization does not lead to the complete disappearance of social ties but changes the basis on which they are formed: they begin to be organized around actions constituting a mere pretext for beingshared-with-others, such as shopping, clubbing or attending mass events. Public space ceases to serve the goal of generating common knowledge and becomes the domain in which emotions and sensations are shared, while communities created through this process acquire a "neo-tribal" character (Maffesoli 1996) or become "peg communities" (Bauman 2001b) that serve merely to "hang" individual identity on a "peg" offered by a specific activity.

Can we consider today's "discreet society" as the seed of a new form of social organization - of a mythologized postmodernity whose shape many attempt to grasp in their predictions about the future? Not exactly, since - as can be gleaned from table 9.1. - the basic parameters of the discreet society differ both from those characterizing the modern society and those ascribed to the postmodern society - ones we have reconstructed from descriptions contained in many studies. In fact, it would be an exaggeration to speak - as far as the discreet society is concerned - about a different type of organization of social life, and especially about a different type of sociality. After all, we deal here, on the 
Table 9.1. Basic parameters of various modes of organizing social life

\begin{tabular}{|c|c|c|c|c|}
\hline & $\begin{array}{l}\text { Traditional } \\
\text { society }\end{array}$ & $\begin{array}{l}\text { Modern } \\
\text { society }\end{array}$ & $\begin{array}{l}\text { Contemporary } \\
\text { society }\end{array}$ & $\begin{array}{l}\text { Features ascribed } \\
\text { to postmodern } \\
\text { society }\end{array}$ \\
\hline $\begin{array}{l}\text { Type of social } \\
\text { organization }\end{array}$ & $\begin{array}{l}\text { society of local } \\
\text { communities }\end{array}$ & $\begin{array}{l}\text { society of large } \\
\text { structures }\end{array}$ & discreet society & $\begin{array}{l}\text { society of regional } \\
\text { communities }\end{array}$ \\
\hline $\begin{array}{l}\text { Dominant type } \\
\text { of community }\end{array}$ & tribe & nation & "neo-tribes" & humanity \\
\hline $\begin{array}{l}\text { Means of } \\
\text { symbolic } \\
\text { integration }\end{array}$ & religion & ideology & mass culture & individual rights \\
\hline $\begin{array}{l}\text { Main principle } \\
\text { of actions }\end{array}$ & survival & $\begin{array}{l}\text { maximization of } \\
\text { profits }\end{array}$ & $\begin{array}{l}\text { consuming } \\
\text { sensations }\end{array}$ & $\begin{array}{l}\text { sustainable } \\
\text { development }\end{array}$ \\
\hline $\begin{array}{l}\text { Dominant type } \\
\text { of order }\end{array}$ & social & economic & political & $\begin{array}{l}\text { harmonious } \\
\text { amalgamation of } \\
\text { orders? }\end{array}$ \\
\hline $\begin{array}{l}\text { Aim of } \\
\text { socialization }\end{array}$ & community & position & objects & people \\
\hline $\begin{array}{l}\text { Mode of social } \\
\text { control }\end{array}$ & culture of shame & culture of guilt & $\begin{array}{l}\text { culture of "being } \\
\text { yourself" }\end{array}$ & $\begin{array}{l}\text { culture of } \\
\text { authenticity }\end{array}$ \\
\hline $\begin{array}{l}\text { Identity- } \\
\text { determining } \\
\text { division }\end{array}$ & $\begin{array}{l}\text { US-THEM } \\
\text { (aliens) }\end{array}$ & $\begin{array}{l}\text { US-THEM } \\
\text { (others) }\end{array}$ & ME-THEM & $M E-U S$ \\
\hline $\begin{array}{l}\text { Dominant type } \\
\text { of relations }\end{array}$ & "natural" bonds & relations & influences & $\begin{array}{l}\text { bonds based on } \\
\text { acknowledged } \\
\text { interdependence }\end{array}$ \\
\hline $\begin{array}{l}\text { Dominant type } \\
\text { of motivation }\end{array}$ & "natural will" & "arbitrary will" & "arbitrary will" & "reflexive will" \\
\hline $\begin{array}{l}\text { Significant } \\
\text { others }\end{array}$ & $\begin{array}{l}\text { elderly as } \\
\text { carriers of } \\
\text { tradition }\end{array}$ & $\begin{array}{l}\text { moral } \\
\text { authorities }\end{array}$ & experts, idols & $?$ \\
\hline $\begin{array}{l}\text { Positioning of } \\
\text { common good }\end{array}$ & $\begin{array}{l}\text { in relation with } \\
\text { nature }\end{array}$ & $\begin{array}{l}\text { in relation with } \\
\text { other imagined } \\
\text { communities }\end{array}$ & $\begin{array}{l}\text { fragmentation of } \\
\text { common good }\end{array}$ & $\begin{array}{l}\text { in relations with } \\
\text { other regional } \\
\text { communities }\end{array}$ \\
\hline
\end{tabular}

one hand, with a form of organization that, in its basic properties, can be identified with the modern society (with large structures still playing a key role, albeit they are oriented today at controlling boundary conditions), while on the other with no signs allowing to ascertain that this form could develop such a mode of integration that would correspond to a global level of coordinating actions. 
It would be possible to go further and claim that the contemporary "discreet society" is characterized by a high level of social disintegration, involving the breaking down of social order and the appearance of what Durkheim has called social anomie, both on the "local" level of countries where processes of transformation have developed to the highest degree, and on the global level of the entire world. In the case of the former, disintegration affects the basic elements of modern form of sociality (one that has dominated so far): the image of the world based on the vision of cooperating social classes, which negotiate the direction of social development within the framework of democratic state institutions. Significant limitations have been imposed on welfare state mechanisms (some even speak of a hasty dismantling), which previously allowed the state to overtake communal functions and then became the basis for structural individualization. Finally, the increasing complexity of modern social life has led to the emergence of a series of phenomena collectively known as "ungovernability." It manifests itself in a general loss of approval for political leadership, intensification of political radicalism, weakening of traditional political parties, development of new social movements, rise in crime rates, related reprivatization of security means and so forth (Hausner 2002).

As far as the global order is concerned, the status quo reached towards the end of the nineteenth century began to undergo significant transformations already in the years preceding the Second World War, and was completely dismantled in the 1960s. Globalization processes, which accelerated towards the end of the twentieth century, were expected by some to create a new mode of integration. However, they still affect only the "material" dimension (economy, ecology, risk). Nothing seems to indicate that they could embrace symbolic and political aspects of life on earth in any foreseeable future. People still inhabit different worlds, with globalization only deepening these differences instead of eliminating them. At the same time, however, thanks to intensification of mass communication, they begin to know more and more about each other. These various worlds - not only from starving Africa to the wasteful USA but also from entirely marginalized enclaves of poverty to relatively slim elites that "rebel," as Christopher Lasch argues (1994), against taking responsibility for the fate of their societies - begin to constitute frames of reference for each other: they have to be taken into account while considering one's own society. Moreover, migrations that assume mass character begin to have specific consequences rightly identified by Roger Scruton (though put in somewhat emphatic terms): "People are being brought into connection who have no real way of accommodating one another, and the spectacle of Western freedom and Western prosperity, going hand-in-hand with Western decadence and the crumbling of Western loyalties, is bound to provoke, 
in those who envy the one and despise the other, a seething desire to punish" $(2002,83)$.

It can be easily noticed that social and political conflicts have intensified in recent years, while state and international institutions are losing their ability to mediate and discipline. We also observe the exhaustion of those resources in the human habitat which rose to such prominence in the twentieth century, particularly the entire infrastructure of social institutions responsible for coordination and peaceful cooperation of individuals within the state, which the civil society (one that emerged at the threshold of modernity) furnished with the task of sustaining the form of sociality which it itself created. The current revival of interest in the eighteenth-century concept of civil society seems directly related to the ever more acutely felt limitation of the possibility to recreate that form of socializing interpersonal relations which provided the foundation for both modern society and sociology as a discipline studying that society. To put it more emphatically, contemporary works referring to the concept of civil society are basically desperately conjuring up the very idea of social integration in the face of the currently progressing disintegration of Western societies. ${ }^{413}$

Moreover, the invariability and permanence of the capitalist form of economybasic argument of those arguing that we deal with a continuation of modernity as the dominant principle of social organization - seem to be closer to wishful thinking rather than an adequate account of reality. This concerns not only processes described in Chapter Six - i.e. ones leading from organized to disorganized capitalism, which themselves cause many changes in social structures - but also, or even primarily, the change of the main source of generating profit. It is no longer production and sale, but time required by global circulation of capital. As

413 Such distribution of emphasis - one that completely differs from the eighteenthcentury sense of power, which permeates considerations on the subject of the budding civil society - is observable both in empirically-grounded accounts that demonstrate what consequences the lowering of civic engagement can have for the functioning of democratic states (cf. e.g. Putnam 2000), and in conceptual analyzes that have a clearly projecting character. The latter also emphasize the necessity for society as such to reconstitute its autonomy from the state, regain its ability to decide about itself - something it lost contemporarily due to the state overtaking most communal tasks and functions. This has been articulated by Edward Shils, who claims that " $[\mathrm{t}] \mathrm{he}$ idea of civil society is the idea of a part of society which has a life of its own, which is distinctly different from the state" $(1997,292)$, and by Jeoffrey Alexander (2002), who explicitly alludes to Durkheim's tradition by distinguishing the civil society as an area that is analytically (and to a certain extent empirically) separate from the areas of politics, economy or religion. 
Manuel Castells writes, "time is critical to the profit-making of the whole system" (2001a, 466). This is so not only because profits from trans-border financial flows depend on "the speed of transactions" (465; emphasis preserved) but also because "a significant and growing number of financial transactions are based on making value out of the capture of future time in present transactions, as in the futures, options, and other derivative capital markets" (466). Castells lists many negative consequences of that shift, including "the destruction in the collective experience of societies of the deferred-gratification pattern of behavior, in favor of the 'quick buck' common ideology [...] and the fundamental damage to the social perception of the correspondence between production and reward, work and meaning, ethics and wealth" (467).

The guarded optimism inspired by scholars such as Marshall McLuhan (1962), who heralded the coming of "global village," or more contemporarily Arjun Appadurai (1996), who emphasizes the potential inherent in new, transnational communities emerging thanks to the development of means of communication (both symbolic and spatial), has been largely suppressed in recent years when it turned out that these new forms of communal life can also take the form of global terrorism. The belief that a postmodern society would hatch, offering a new form of social integration that could embrace entire humanity, is basically kept alive today solely by the conviction that people must acknowledge the fact that it is humanity as such - not individual societies enclosed within the boundaries of nation states - that currently constitutes the basic unit of survival. Risks analyzed by Beck have a global character now and no nation state can appropriately deal with them on its own.

However, such convictions seem to be poorly grounded in reality because currently existing human collectivities fundamentally differ in many respects: their social strength (i.e. the ability to secure means for survival or expansion), the character of overarching social organization (hunter-gatherer societies coexist alongside agricultural, industrial and post-industrial ones), and political solutions (coexistence of probably all historically identified forms of government). Another difference concerns "collective representations" meant to sustain integration and mobilize communities: both representations of intra-group relations (religious Integrism vs individualized society based on the acknowledging of individual rights) and representations of inter-group relations (multiculturalism and international cooperation vs nationalism and defense of sovereignty).

One could thus say that, although the globalized world keeps transforming under the influence of "flows" that encircle it - as suggestively described by Castells (2001a) - it seems unjustified to expect that this process will itself generate a global form of sociality in which humanity would become the superior 
"imagined community." Instead, there are reasonable grounds to fear that these "flows" could turn into a gigantic and uncontrollable tsunami.

\subsection{What comes after modernity?}

Regardless of the level we choose to start describing the contemporary society, it seems clear that the organization of social life is undergoing intense transformations in all of its dimensions. What is more, most of these changes have a destructive rather than a constructive character. They manifest themselves primarily in the disappearance of previous institutional forms rather than in the emergence of new ones. As we have demonstrated, the contemporary family has lost most of its primary functions and its existence becomes increasingly reliant on an emotional sense of self-fulfillment among individuals. Work is becoming a rare good. It has also ceased to position individuals in social space. Institutions characteristic for modern public space are losing their ability to produce common knowledge, which has been undergoing fragmentation, giving rise to a series of aspectual and incompatible worldviews created within the framework of online communication.

The scope and depth of the currently experienced transformations evidently indicate that we have entered a period of formative transition. ${ }^{414}$ Although the "discreet" society still carries numerous characteristics of the modern one, its most prominent feature is not related to processes of change or crisis but to ones involving the destruction of the basic pillars supporting the former social order. One could even argue that, just like in the case of the transition to modernity, the dismantling of old (i.e. modern) forms of sociality appears to be a necessary stage preceding the emergence of new ones. At the same time, we should remember that one vital feature of changes accompanying the transition from one form organizing social life to another is their abrupt character (although the process would be stretched in time) and non-cumulative nature (Nisbet 1972): a new social order does not emerge from prior slight changes and mutual adaptations occurring in various areas of social life, but rather from the chaos of preceding actions that are the outcome of - on the one hand - a decreasing efficiency of earlier forms of sociality, and on the other - the search for new forms that would be better suited to the changing reality. This causes the periods of formative transition to display a high degree of anomie.

414 From among a number of different dates marking the beginning of a new era we have chosen the 1970s as the period when new phenomena have begun to manifest themselves in a distinctive way. 
Already at the beginning of the 1980s, Ralph Dahrendorf would write about the contemporary society in the following way: "Anomie has become an element of the lives of many and notably of those who are still on the way of becoming full members of their societies; the teaching of history is reduced; the church becomes a christening and burying institution; mobility is a higher value than local loyalties; many families have cracked or frozen" $(1981,42)$. Dahrendorf sought the causes of these transformations in the downfall of a specific type of social bond linking individuals to groups they find important because of the meaning such groups give to the place they occupy in society, obliging them to remain loyal. ${ }^{415}$ In his view, contemporary society is characterized by an immense increase of choices, accompanied by the dissolution of the kind of bonds that would guide these individual choices, to which he refers with the term "ligatures." As he argues, in contemporary societies "the reduction, and ultimately destruction of bonds has been accompanied by, and perhaps been responsible for, an increase of choices up to a point; but from this point onwards choices begin to lose their meaning because they take place in a social vacuum, or better perhaps, a social desert, in which no known co-ordinates make any direction preferable to any other" (31-32).

One consequence of the increase of choices at the cost of the dissolution of bonds involves - as Dahrendorf claims - the currently observable transformations of society. They would unveil gradually since the reduction of ligatures "began by extending the importance of achieved social positions but in the end it turned against all ascribed positions, whatever their basis. For some, being a Christian begins to mean not having any distinctive faith; women's liberation begins to mean that there must be no social difference between men and women; 'grey panthers' demand the abolition of old age as children's liberationists have demanded the abolition of childhood. [...] The destruction of ligatures in significant parts of some societies has begun to lead to a reduction of complexity which in turn involves a decrease in life chances: the choices themselves disappear which a modern society was meant to offer to all" (36). ${ }^{416}$

415 Such groups could include local communities, professional circles, homeland, ancestors, etc. Although Dahrendorf does not articulate this explicitly, the context of his discussion would indicate that he means groups with which the individual would strongly identify, embracing their norms and values.

416 Obliteration of all differences stemming from social affiliations would mean that individuals become "pure," i.e. not determined by any coordinates of social identity, which could provide a basis for making choices. If my decisions are unaffected by the fact that I am a man or a woman, a child or an old person, a Catholic, a Pole, a liberal, etc., then what could help me orient myself in social space? Merely biological drives or psychological moods. 
The dissolution of social bonds was both cause and effect of those changes occurring in areas of economy, politics and society, which paved the way for processes of structural individualization and gave rise to the "discreet" society. Therefore, calls for reconstructing community have become the fundamental reaction to the directly experienced increase of social anomie. As Paul Morris observed in the mid-1990s, "[e]veryone, but everyone, is for community. Our politicians, of the old and the new, Left and Right; our political philosophers; our religious leaders; our modern and postmodern theorists appear to share little but their pious promotion of 'community.' The demands on us to (re)create community are incessant and ubiquitous. We are besieged by calls to support community: our local community; our regional community; our national community; the European Community, and the international community" (1996, 223).

From a sociological perspective, such calls are merely attempts to put a spell on a reality that we no longer control. Referencing the mythologized community, they entirely disregard the fact that communities are not intentionally willed into existence by their members but rather constitute the effect of being-shared-withothers, rising out of cooperation based on shared beliefs, norms and aspirations. Thus, the dissolution of bonds experienced today stems not from people's "reluctance" to join communities but from the collapse of those beliefs, norms and aspirations that integrated their actions into some kind of social organization subordinated to the achievement of some higher goal.

For traditional societies this higher goal was to sustain and recreate the order established by ancestors or gods, whereas for modern societies it was progress. These kinds of goals provided a framework for individual desires and actions. In the former case, individuals would be rewarded by joining their ancestors or being saved, while in the latter - by achieving individual success. Salvation or success could be (theoretically) earned by everyone insofar as they would conform to norms constituting a particular axiological order. At the same time, this caused individual aspirations to legitimize the system and become part of processes aiming to reproduce it.

The collapse of the idea of progress in the twentieth century created an axiological void that could not be filled with calls to recreate community. The rapid growth of anti-system concepts has not been accompanied so far by the emergence of any idea or image that would prove capable of integrating individual actions so as to facilitate the emergence of new, global social structures. One could only indicate that the category of humanity as a potentially higher imagined community would demand - on the one hand - accepting its internal diversity, and on the other - remodeling our relationship with Earth as our global habitat, one on which the survival of humans depends. Although at the current 
stage of the formative transition period it seems highly unlikely that any such idea or image could be created (and accepted), one needs to remember that it would have seemed equally improbable to predict, at the beginning of the transition to modernity, that the Protestant ethos rooted in the idea of predestination could later evolve into an idea of a success that everyone can achieve.

It seems equally impossible to predict what shape the new forms of sociality might take. Just like sixteenth- or even seventeenth-century intellectuals could not imagine a social order without monarchy, distinct social estates sanctioned by tradition, and dominance of religion, we also find it challenging to imagine how today's social structure might change, how decisions affecting larger collectivities would be made, and what would be the arguments justifying individual actions. At best, we can assume that it is highly probable that the forms of organizing social life, which will emerge at the end of the postmodern transition, are bound to be entirely different from those that we consider to be obvious today. ${ }^{417}$

We might also recall the words of Jean-Luc Nancy, who pointed to the frantic search for ways to reconstruct community as a key characteristic of contemporaneity: "Society was not built on the ruins of a community. It emerged from the disappearance or the conservation of something - tribes or empires - perhaps just as unrelated to what we call 'community' as what we call 'society.' So that community, far from being what society has crushed or lost, is what happens to us - question, waiting, event, imperative - in the wake of society." He concludes by arguing that "[n]othing, therefore, has been lost, and for this reason nothing is lost. We alone are lost, we upon whom the 'social bond' (relations, communication), our own invention, now descends heavily like the net of an economic, technical, political, and cultural snare. Entangled in its meshes, we have wrung for ourselves the phantasms of the lost community" (1991, 11-12; emphasis preserved).

417 The scope of possible solutions is determined here, on the one hand, by achieving full social integration on the global level, which would necessitate (as the model adopted here requires) creating new, global mechanisms of integrating and coordinating individual actions, and on the other - by the fragmentation of the world into areas occupied by communities not necessarily based on nations but characterized by distinct forms of sociality, ones fighting or ignoring each other. One example of such a solution is provided by Mormon or Amish communities, which coexist in American society with individualized communities of the West and East Coasts as well as with more traditional communities of the interior states, although each community of this kind has to occupy a separate, self-governed territory. 
One only needs to remember that setting oneself free from the social bond that has laid at the foundation of the modern society does not entail the emergence of a "sovereign individual" who would shape his or her aspirations and actions in the course of free reflection, entirely liberated from any social influence. Whether we like it or not, we are the "product" of those forms of socializing individual actions in which we participate and which we sometimes initiate ourselves. Perhaps, the point is rather to start developing a new type of bond in the globalized world by engaging in activities that would aim to solve that world's problems, thus creating a new framework for being-shared-with-others - the indispensable condition for the existence of any human community. 


\section{REFERENCES}

Abbott, Andrew. 1992. "From Causes to Events. Notes on Narrative Positivism." Sociological Methods and Research 20.4: 428-455.

Adorno, Theodor W., E. Frenkel-Brunswik, D. J. Levinson \& R. N. Sanford.

1950. The Authoritarian Personality. New York: Harper \& Brothers.

Alexander, Jeffrey C. 2002. "Discourse and Civil Society. disClouser Interviews

J. Alexander." disClouser: a Journal of Social Theory 12.

Anderson, Benedict. 2006. Imagined Communities. Reflections on the Origin and Spread of Nationalism. London: Verso.

Anthony, P. D. 1977. The Ideology of Work. London: Tavistock.

Appadurai, Arjun. 1996. Modernity at Large. Cultural Dimensions of

Globalization. Minneapolis: University of Minnesota Press.

Arendt, Hannah. 1998. The Human Condition. Chicago: University of Chicago Press.

Ariès, Philippe. 1965. Centuries of Childhood: A Social History of Family Life.

New York: Random House.

Ariès, Philippe. 1989. "Introduction." In: History of Private Life, vol. 3, Passions of the Renaissance. Edited by Roger Chartier. Translated by Arthur Goldhammer. Cambridge, Mass.: The Belknap Press of Harvard University Press.

Aronson, Elliot, Timothy D. Wilson, Robin M. Akert \& Samuel R. Sommers. 2015. Social Psychology. Harlow: Pearson.

Bandura, Albert \& Richard H. Walters. 1959. Adolescent Aggression.

New York: Ronald Press.

Bandura, Albert \& Richard H. Walters. 1967. Social Learning and Personality Development. New York: Holt, Rinehart \& Winston.

Barraclough, Geoffrey. 1969. An Introduction to Contemporary History. Harmondsworth: Penguin Books.

Baszkiewicz, Jan. 1999. Władza. Wrocław: Zakład Narodowy im. Ossolińskich. Bauman, Zygmnut. 1972. "A Note on Mass Culture: On Infrastructure."

Sociology of Mass Communication, Edited by D.McQuail. Hammondsworth: Penguin Books.

Bauman, Zygmunt. 1988. Freedom. Milton Keynes: Open University Press.

Bauman, Zygmunt. 1998a. "Zbędni, niechciani, odtrąceni - czyli o biednych w zamożnym świecie.” Kultura i Społeczeństwo 2: 3-18. 
Bauman, Zygmunt. 1998b. “Zawrotna kariera 'podklasy"” Dziś. Przegląd Społeczny 1-2: http://portal.tezeusz.pl/2004/11/27/zawrotna-kariera-podklasy/.

Bauman, Zygmunt. 2001a. The Individualized Society. Cambridge: Polity Press. Bauman, Zygmunt. 2001b. “On Mass, Individuals, and Peg Communities."

In: N. Lee \& R. Munro (eds.), The Consumption of Mass, 102-113. Oxford: Blackwell.

Bauman, Zygmunt. 2003. Intimations of Postmodernity. London: Routledge.

Bauman, Zygmunt. 2006. Liquid Modernity. Cambridge: Polity.

Bauman, Zygmunt. 2008. Liquid Love. On the Frailty of Human Bonds. Cambridge: Polity.

Bauman, Zygmunt. 2010. Towards a Critical Sociology. New York: Routledge.

Beck, Ulrich. 1992. Risk Society. Towards a New Modernity. Translated by Mark Ritter. London: Sage.

Beck, Ulrich \& Elisabeth Beck-Gernsheim. 1996. "Individualization and 'Precarious Freedom': Perspectives and Controversies of a Subject-oriented Sociology.” In: P. Heelas, S. Lash, P. Morris (eds.), Detraditionalization. Critical Reflections on Authority and Identity. Cambridge Mass.: Blackwell Publishers.

Becker, Gary S. 1991. A Treatise on the Family. Cambridge: Harvard University Press.

Bell, Daniel. 1973. The Coming of Post-Industrial Society. New York: Basic Books.

Bell, Daniel. 1976. The Cultural Contradictions Of Capitalism. New York: Basic Books.

Benedict, Ruth. 2005. The Chrysanthemum and the Sword: Patterns of Japanese Culture. New York: Mariner Books.

Benhabib, Seyla. 1992. Models of Public Space: Hannah Arendt, the

Liberal Tradition and Jürgen Habermas. In: Situating the Self: Gender,

Community and Postmodernism in Contemporary Ethics, 89-120.

Cambridge: Polity Press.

Berger, Peter L. 1967. The Sacred Canopy: Elements of a Sociological Theory of Religion. New York: Doubleday.

Berger, Peter L. \& Thomas Luckmann. 1991. The Social Construction of Reality. Harmondsworth: Penguin Books.

Bernstein, Basil. 1971. Class, Codes, and Control, vol. 1. London: Paladin.

Bielicki, Marian. 1966. Zapomniany świat Sumerów. Warsaw: Państwowy Instytut Wydawniczy. 
Blackmore, Susan. 2000. The Meme Machine. Oxford: Oxford University Press. Blumer, Herbert. 1966. "Sociological Implications of the Thought of George Herbert Mead." American Journal of Sociology 71.5: 535-544.

Bogucka, Maria. 1998. Białogłowa w dawnej Polsce. Warsaw: Trio.

Bourdieu, Pierre. 1972. "Les strategies matrimoniales dans le systeme de reproduction.” Annales E.S.C. (July-October): 1105-1127.

Bourdieu, Pierre. 1979. Distinction: A Social Critique of the Judgment of Taste. London: Routledge.

Bourdieu, Pierre. 1986. “The Forms of Capital.” In: Handbook of Theory and Research for Sociology of Education. Edited by John G. Richardson. New York: Greenwood Press.

Bourdieu, Pierre \& Jean-Claude Passeron. 2000. Reproduction in Education, Society and Culture. London: Sage Publications.

Braudel, Fernand. 1983. Civilization and Capitalism, 15th-18th Century, Volume II: The Wheels of Commerce. Translated by Siân Reynold. London: Book Club Associates.

Braudel, Fernand. 1992. Civilization and Capitalism, 15th-18th Century, Volume III: The Perspective of the World. Translated by Siân Reynold. Berkeley: University of California Press.

Brenner, Robert. 1989. "Economic Backwardness in Eastern Europe in Light of Developments in the West." In: The Origins of Backwardness in Easter Europe. Economics and Politics from the Middle Ages until the Early Twentieth Century, Edited by D. Chirot. Berkeley: University of California Press.

Bronowski, Jacob. 1979. The Origins of Knowledge and Imagination. New Haven: Yale University Press. [Quoted after the 1984 Polish translation by S. Amsterdamski: Źródła wiedzy i wyobraźni. Warszawa: PIW.]

Bronowski, Jacob. 2011. The Ascent of Man. London: BBC Books. E-book. Budnikowski, Tomasz. 2002. Bezrobocie na świecie i w Polsce. Poznań: Instytut Zachodni.

Burgess, Ernest W. \& Harvey J. Locke. 1945. The Family: From Institution to Companionship. New York: American Book Company.

Cabantous, Alain. 1990. "La fin des patriarches." In: J. Delumeau \& D. Roche (eds.), Histoire des pères et de la paternité. Paris: Larousse. [Quoted after the 1995 Polish translation by J. Radożycki \& M. Paloetti-Radożycka: J. Delumeau \& D. Roche (eds.), Historia ojców i ojcostwa, 323-349. Warsaw: Volumen.]

Camic, Charles M. 1987. “The Making of the Method: A Historical Reinterpretation of the Early Parsons." American Sociological Review 52.4: 421-439. 
Caporael, Linnda. 1995. "Sociality: Coordinating Bodies, Minds and Groups." Psycoloquy 6.1. http://www.cogsci.ecs.soton.ac.uk/cgi/psyc/newpsy?6.01.

Castaneda, Carlos. 1972. Journey to Ixtlan: The Lessons of Don Juan.

New York: Simon \& Schuster.

Castells, Manuel. 2001a. The Rise of the Network Society. Oxford: Blackwell.

Castells, Manuel. 2001b. End of Millenium. Oxford: Blackwell.

Castells, Manuel. 2002. The Power of Identity. Oxford: Blackwell.

Charbonnier, Georges. 1969. Conversations with Claude Lévi-Strauss.

Translated by John \& Doreen Weightman. London: Jonathan Cape.

Chaunu, Pierre. 1971. La Civilisation de l'Europe des Lumières. Paris: Arthaud.

Chojnowska, Alina. 2002. Psychospołeczne konsekwencje przemian pracy we wspótczesnym kapitalizmie. MA thesis. Insititute of Sociology at the University of Warsaw.

Chouraqui, André. 1971. La vie quotidienne des Hébreux au temps de la Bible. Paris: Hachette.

Chwe, Suk-Young M. 2001. Rational Ritual. Culture, Coordination, and Common Knowledge. Princeton: Princeton University Press.

Cole, Michael \& Yrjö Engeström. 2001. "A Cultural-historical Approach to Distributed Cognition." In: Distributed Cognitions. Psychological and Educational Considerations. Edited by: G. Salomon. Cambridge: Cambridge University Press.

Collins, Randall. 1975. Conflict Sociology. Toward an Explanatory Science. New York: Academic Press.

Comte, August. 2009. A General View of Positivism. Cambridge: Cambridge University Press.

Crozier, Michel \& Erhard Friedberg. 1980. Actors and Systems: The Politics of Collective Action. Chicago: University of Chicago Press.

Czarnowski, Stefan. 1956. Kultura. Warsaw: Książka i Wiedza.

Dahrendorf, Ralf. 1981. Life Chances. Approaches to Social and Political Theory. Chicago: University of Chicago Press.

Daly, Martin \& Margo Wilson. 1988. Homicide. New York: Aldine de Gruyter.

Damasio, Antonio R. 1995. Descartes' Error. Emotion, Reason, and the Human Brain. New York: Avon Books.

Deacon, Terence W. 1997. The Symbolic Species: The Co-evolution of Language and the Brain. New York: Norton.

Dennett, Daniel C. 1996. Kinds of Minds. New York: Harper Collins. 
Diamond, Jared. 1991. The Rise and Fall of the Third Chimpanzee. London: Radius.

Diamond, Jared. 1997. Why is Sex Fun? The Evolution of Human Sexuality. New York: Basic Books.

Diamond, Jared. 1999. Guns, Germs and Steel. The Fates of Human Societies. New York: W. W. Norton \& Company.

Drucker, Peter. F. 1994. Post-Capitalist Society. New York: HarperCollins.

Duby, Georges. 1988. "Introduction.” In: A History of Private Life, vol. 2, Revelation of the Medieval World. Edited by Georges Duby. Translated by Arthur Goldhammer. Cambridge, Mass.: The Belknap Press of Harvard University Press.

Duby, Georges. 1997-1998. Women of the Twelfth Century, vols. 1-3. Translated by Jean Birrell. Chicago: University of Chicago Press.

Duby, Georges. 1998. Polish introduction to Historia życia prywatnego, vol. 2, Od Europy feudalnej do renesansu, 17-44. Translated by W. Bieńkowska. Wrocław: Zakład Narodowy im. Ossolińskich.

Dunbar, Robin. 1996. Grooming, Gossip and the Evolution of Language. Cambridge: Harvard University Press.

Durkheim, Émile. 1981. “The Realm of Sociology as a Science." Translated by Everett K. Wilson. Social Forces 59.4: 1054-1070.

Durkheim, Émile. 1982. The Rules of Sociological Method. Translated by W.D. Halls. New York: The Free Press.

Durkheim, Émile. 2001. The Elementary Forms of Religious Life. Translated by Carol Cosman. Oxford: Oxford University Press.

Durkheim, Émile. 2013. The Division of Labour in Society. Translated by W.D. Halls. Basingstoke: Palgrave Macmillan.

Edelman, Gerald M. 1992. Bright Air, Brilliant Fire: On The Matter Of The Mind. New York: Basic Books.

Eliade, Mircea. 1989. Shamanism: Archaic Techniques of Ecstasy. Translated by Williard R. Trask. Harmondsworth: Penguin Books.

Elias, Norbert. 1978. What is Sociology? Translated by Stephen Mennell \& Grace Morrissey. London: Hutchinson.

Elias, Norbert. 2000. The Civilizing Process. Translated by Edmund Jephcott. Malden: Blackwell.

Elias, Norbert. 2001. The Society of Individuals. Translated by Edmund Jephcott. London: Continuum.

Elias, Norbert. 2007. Involvement and Detachment. Translated by Michael Schröter. Dublin: University College Dublin Press. 
Emirbayer, Mustafa \& Ann Mische. 1998. "What is Agency?” American Journal of Sociology 103.4: 962-1023.

Finch, Janet. 1989. Family Obligations and Social Change. Cambridge: Polity.

Fisher, Helen. 1994. Anatomy of Love: A Natural History of Mating, Marriage, and Why We Stray. New York: Ballantine Books.

Fitoussi, Jean-Paul \& Pierre Rosanvallon. 1998. Le Nouvel Âge Des Inégalités. Paris: Seuil.

Flandrin, Jean Louis. 1980. Families in Former Times. Cambridge: Cambridge University Press.

Foucault, Michel. 1979. Discipline and Punish. The Birth of the Prison. Translated by Alan Sheridan. New York: Vintage.

Foucault, Michel. 1988-1990. The History of Sexuality. Volumes 1-3. Translated by Robert Hurley. New York: Vintage.

Frankfort-Nachmias, Chava \& David Nachmias. 2008. Research Methods in the Social Sciences. New York: Worth Publishers.

Frieske, Kazimierz W. 2004. "Dynamika zbiorowych stosunków pracy - o tym, że Giambattista Vico miał rację." In: Przyszłość pracy w XXI wieku. Edited by S. Borkowska. Warsaw: IPiSS, GUS.

Gauchet, Marcel. 1999. The Disenchantment of the World. A Political History of Religion. Translated by Oscar Burge. Princeton: Princeton University Press.

Gauchet, Marcel. 2000. "A New Age of Personality: An Essay on the Psychology of Our Times." Translated by Johann Arnason \& David Roberts. Thesis Eleven 60: 23-41.

Gawin, Magdalena. 2003. Rasa i nowoczesność. Historia polskiego ruchu eugenicznego. Warsaw: Neriton \& Instytut Historii PAN.

Gehlen, Arnold. 2001. W kregu antropologii i psychologii społecznej. Studia. Translated by K. Krzemieniowa. Warsaw: Czytelnik. [For the German original see: Gehlen, Arnold. 1993. Anthropologische und sozialpsychologische Untersuchungen. Hamburg: Rowohlt Tb.]

Giddens, Anthony. 1986. The Constitution of Society. Outline of the Theory of Structuration. Cambridge: Polity Press.

Giddens, Anthony. 2006. Modernity and Self-Identity. Self and Society in the Late Modern Age. Cambridge: Polity Press.

Gillis, John R. 1974. Youth and History. London: Academic Press.

Gilmore, David D. 2001. Misogyny. The Male Malady. Philadelphia: University of Pennsylvania Press. 
Giza-Poleszczuk, Anna. 1991a. "Kultura społeczna." In: Co nam zostało z tych lat... Społeczeństwo polskie u progu zmiany systemowej. Edited by M. Marody. London: Aneks.

Giza-Poleszczuk, Anna. 1991b. "Stosunki międzyludzkie i życie zbiorowe." In: Co nam zostało $z$ tych lat... Społeczeństwo polskie u progu zmiany systemowej. Edited by M. Marody. London: Aneks.

Giza-Poleszczuk, Anna. 2000. "Przestrzeń społeczna." In: Strategie i system. Polacy w obliczu zmiany systemowej. Edited by A. Giza-Poleszczuk, M. Marody, A. Rychard. Warsaw: Wydawnictwo IFiS PAN.

Giza-Poleszczuk, Anna, Mirosława Marody \& Andrzej Rychard (eds.). 2000. Strategie i system. Polacy w obliczu zmiany systemowej. Warsaw: Wydawnictwo IFiS PAN.

Giza-Poleszczuk, Anna. 2002. "Związki partnerskie, małżeństwo i dzieci kulturowe zróżnicowanie postaw.” In: A. Jasińska-Kania \& M. Marody (eds.), Polacy wśród Europejczyków. Warsaw: Scholar.

Giza-Poleszczuk, Anna. 2005. Rodzina a system społeczny: reprodukcja $i$ kooperacja w perspektywie interdyscyplinarnej. Warsaw: Wydawnictwa Uniwersytetu Warszawskiego.

Goffman, Erving. 1967. Interaction Ritual. Essays on Face-to-Face Behavior. New York: Doubleday.

Goldfinger, Charles. 1998. Travail et hors-travail - vers une société fluide. Paris: Editions Odile Jacob.

Goleman, Daniel. 1995. Emotional intelligence. New York: Bantam Books.

Goody, Jack \& I. P. Watt. 1972. “The Consequences of Literacy.” In: Language and Social Context. Edited by P. P. Giglioli. Harmondsworth: Penguin Books.

Goody, Jack. 1978. The Domestication of the Savage Mind. Cambridge: Cambridge University Press.

Goody, Jack. 1983. The Development of the Family and Marriage in Europe. Cambridge: Cambridge University Press.

Gould, Stephen Jay. 1989. Wonderful Life. The Burgess Shale and the Nature of History. New York: Norton.

Graves, Robert. 1992. The Greek Myths. Harmondsworth: Penguin Books.

Greenfield, Patricia Marks \& Jerome S. Bruner. 1966. "Culture And Cognitive Growth.” International Journal of Psychology 2.1: 89-107.

Greenspan, Stanley \& Beryl Lieff Benderly. 1997. The Growth of the Mind, and the Endangered Origins of Intelligence. Cambridge: Perseus Books.

Gromkowska, Agnieszka. 2002. Kobiecość w kulturze globalnej. Rekonstrukcje i reprezentacje. Poznań: Wolumin. 
Guille-Escuret, Georges. 1994. Le Decalage humain. Le fait social dans l'evolution. Paris: Kime.

Gurevich, A.J. 1985. Categories of Medieval Culture. Translated by G.L. Campbell. London: Routledge \& Kegan Paul.

Habermas, Jürgen. 1979. Communication and the Evolution of Society.

Translated by Thomas McCarthy. Boston: Beacon Press.

Habermas, Jürgen. 1987a. The Theory of Communicative Action, vol. 1., Reason and the Rationalization of Society. Translated by Thomas McCarthy. Cambridge: Polity.

Habermas, Jürgen. 1987b. The Theory of Communicative Action, vol. 2., Lifeworld and System: A Critique of Functionalist Reason. Translated by Thomas McCarthy. Cambridge: Polity.

Habermas, Jürgen. 1989. The Structural Formation of the Public Sphere: An Inquiry into a Category of Bourgeois Society. Translated by Thomas McCarthy. Cambridge: MIT Press.

Habermas, Jürgen. 1998. The Philosophical Discourse of Modernity. Translated by Frederick Lawrence. Cambridge: Polity.

Haidt, Jonathan. 2012. The Righteous Mind. New York: Pantheon. E-book. Hajnal, John. 1965. "European Marriage Patterns in Perspective." In: D. V. Glass \& D.E.C. Eversley (eds.), Population in History. London: Edward Arnold Publishers.

Hartley, Jean, Dan Jackobson, Bert Klandermans \& Tinka van Vuuren. 1991. Job Insecurity - Coping with Jobs at Risk. London: Sage.

Harvey, David. 1995. The Condition of Postmodernity. An Enquiry into the Origins of Cultural Change. Cambridge: Blackwell.

Hausner, Jerzy. 2002. "Od idealnej biurokracji do zarządzania publicznego." In: Wymiary życia społecznego. Polska na przełomie XX i XXI wieku. Edited by M. Marody. Warsaw: Scholar.

Heisenberg, Werner. 1979. Ponad granicami. Translated by K. Wolicki. Warsaw: Państwowy Instytut Wydawniczy.

Hobbes, Thomas. 1998. Leviathan. Edited by J.C.A. Gaskin. Oxford: Oxford University Press.

Hobhouse, Henry. 2005. Seeds of Change: Six Plants That Transformed Mankind. Washington: Counterpoint.

Hsieh, Tony. 2010. Delivering Happiness: A Path to Profits, Passion, and Purpose. New York: Grand Central.

Huizinga, Johan. 1987. The Waning of the Middle Ages. Translated by F. Hopman. London: Penguin Books. 
Hull, David L. 1990. Science as a Process. An Evolutionary Account of the Social and Conceptual Development of Science. Chicago: University of Chicago Press.

Humphrey, Nicholas. 1974. "Species and Individuals in the Perceptual World of Monkeys.” Perception 3: 105-114.

Humphrey, Nicholas. 1998. "Cave Art, Autism, and the Evolution of the Human Mind." Cambridge Archaeological Journal 8.2: 165-191.

Hunt, Lynn \& Catherine Hall. 1990. “The Curtain Rises.” In: A History of Private Life, vol. 4, From the Fires of Revolution to the Great War, 7-94. Edited by Michel Perrot. Translated by Arthur Goldhammer. Cambridge, Mass.: The Belknap Press of Harvard University Press.

Hutchins, Edwin. 2000. “The Cognitive Consequences of Patterns of Information Flow." Intellectica 1.30: 53-74.

Inglehart, Ronald. 1990. Culture Shift in Advanced Industrial Society. Princeton: Princeton University Press.

Jacobson-Widding, Anita. 1983. Identity: Personal and Sociocultural. Uppsala: University of Uppsala.

James, William. 2004. Varieties of Religious Experience. London: Routledge. Joas, Hans \& Wolfgang Knöbl. 2009. Social Theory. Twenty Introductory Lectures. Translated by A. Skinner. Cambridge: Cambridge University Press.

Kaufmann, Jean-Claude. 2001. Ego. Pour une sociologie de l'individu. Paris: Nathan. [Quotations after the 2004 Polish edition translated by K. Wakar. Warsaw: Oficyna Naukowa.]

Knorr-Cetina, Karin. 1997. "Sociality with Objects. Social Relations in Postsocial Knowledge Societies." Theory, Culture and Society 14.4: 1-30.

Kochanowicz, Jacek. 2003. “Trendy cywilizacyjne." In: Wymiary życia społecznego. Polska na przełomie XX i XXI wieku. Edited by M. Marody. Warsaw: Scholar.

Kohlberg, Lawrence. 1981. Essays in Moral Development, vol. 1. San Francisco: Harper \& Row.

Kohn, Melvin L. 1959. "Social Class and Parental Values." American Journal of Sociology 64.4: 337-351.

Kohn, Melvin L. \& Carmi Schooler. 1983. Work and Personality: An Inquiry into the Impact of Social Stratification. Norwood: Ablex Publishing.

Konecki, Krzysztof. 1992. "Nowi pracownicy a kultura organizacyjna. Studium folkloru fabrycznego." Folia Sociologica 24. Łódź: Wydawnictwo Uniwersytetu Łódzkiego.

Koralewicz-Zębik, Jadwiga. 1982. "Wartości rodzicielskie a stratyfikacja społeczna." Studia Socjologiczne 3-4: 225-238. 
Kozek, Wiesława. 2000. "Praca." In: Encyklopedia Socjologii, vol. 3. Warsaw: Oficyna Naukowa.

Kozielecki, Jerzy. 2000. "Kognitariusze po proletariuszach." Rzeczpospolita, 16 December 2000.

Kuhn, Thomas S. 1996. The Structure of Scientific Revolutions. Chicago: The University of Chicago Press.

Kula, Witold. 1983. Teoria ekonomiczna ustroju feudalnego. Warsaw: Książka i Wiedza.

Kumar, Krishan. 1995. From Post-Industrial to Post-Modern Society. New Theories of the Contemporary World. Oxford: Blackwell.

Kymlicka, Will. 1995. Multicultural Citizenship. Oxford: Clarendon Press.

Kymlicka, Will. 2002. Contemporary Political Philosophy: An Introduction. Oxford: Oxford University Press.

Lasch, Christopher. 1977. Haven in a Heartless World: The Family Besieged. New York: Basic Books.

Lasch, Christopher. 1994. The Revolt of the Elites: And the Betrayal of Democracy. New York: W.W.Norton \& Company.

Lash, Scott \& John Urry. 1993. The End of Organized Capitalism.

Cambridge: Polity Press.

Lash, Scott \& John Urry. 1994. Economies of Signs and Space. London: Sage.

Laslett, Peter. 1972. Household and Family in Past Time. Cambridge: Cambridge University Press.

Le Bon, Gustave. 2001. The Crowd. A Study of the Popular Mind. Mineola: Dover Publications.

Le Goff, Jacques. 1970. Kultura średniowiecznej Europy. Translated by H. Szumańska-Grossowa. Warsaw: PWN.

Le Goff, Jacques. 1990. Your Money or Your Life. Economy and Religion in the Middle Ages. Translated by Patricia Ranum. New York: Zone Books.

Le Goff, Jacques. 1992. Medieval Civilization 400-1500. Translated by Julia Barrow. Oxford: Blackwell.

Lelong, Charles. 1963. La Vie Quotidienne En Gaule À Lépoque Mérovingienne.

Paris: Hachette. [Quoted after the 1967 Polish translation by E.

Bąkowska: Życie codzienne w Galii Merowingów. Warszawa: Państwowy Instytut Wydawniczy.]

Lévi-Strauss, Claude. 1985. The View from Afar. Translated by Joachim

Neugroschel \& Phoebe Hoss. Chicago: University of Chicago Press.

Levine, David Z. 1977. Family Formation in an Age of Nascent Capitalism.

New York: Academic Press. 
Levine, David Z. 1987. Reproducing Families. The Political Economy of English Population History. Cambridge: Cambridge University Press.

Lewis-Williams, David. 2011. The Mind in the Cave. Consciousness And The Origins Of Art. London: Thames \& Hudson. E-book.

Łoś, Maria. 1976. “Teoria grup odniesienia - propozycja modyfikacji zakresu pojęcia.” Studia Socjologiczne 4: 107-115.

Maciejewski, Marek. 2001. Powszechne dzieje gospodarcze od końca XV wieku do 1939 roku. Wrocław: Wydawnictwo Uniwersytetu Wrocławskiego.

MacKinnon, Catharine A. 1991. Toward a Feminist Theory of State. Cambridge: Harvard University Press.

Maffesoli, Michel. 1996. The Time of Tribes. The Decline of Individualism in Mass Society. Translated by D. Smith. London: Sage.

Malinowski, Bronisław. 1936. "The Problem of Meaning in Primitve Languages." In The Meaning of Meaning, 296-336. Edited by C.K. Ogden \& I.A. Richards. New York: Harcourt, Brace \& World.

Marody, Mirosława. 1987. Technologie intelektu. Jezykowe determinanty wiedzy potocznej i ludzkiego działania. Warsaw: Państwowe Wydawnictwo Naukowe.

Marody, Mirosława. 1991. “Działania jednostek a system społeczny.” In: Co nam zostało z tych lat... Społeczeństwo polskie u progu zmiany systemowej. Edited by M. Marody. London: Aneks.

Marody, Mirosława. 1996. "Pojęcie wartości w wyjaśnianiu zachowań społecznych." In: Podstawy życia społecznego w Polsce, Edited by M. Marody \& E. Gucwa-Leśny. Warsaw: Instytut Studiów Społecznych UW.

Marody, Mirosława. 2000. "Kulturowe aspekty zmiany społecznej.” In: Między rynkiem a etatem. Społeczne negocjowanie polskiej rzeczywistości. Edited by M. Marody. Warsaw: Scholar.

Marody, Mirosława \& Jerzy Wilkin (eds.). 2003. Na prostej? Polska w przededniu członkostwa w UE. EU-monitoring VII. Kraków: Małopolska Szkoła Administracji Publicznej.

Marx, Karl \& Frederick Engels. 1988. "Manifesto of the Communist Party." In: Karl Marx, Economic and Philosophic Manuscripts of 1844, and the Communist Manifesto. Translated by Martin Milligan. New York: Prometheus.

Mauss, Marcel. 1973. "Techniques of the Body." Translated by Ben Brewster. Economy and Society 2.1: 70-88.

McLuhan, Marshall. 1962. The Gutenberg Galaxy: The Making of Typographic. Toronto: University of Toronto Press. 
McLuhan, Marshall. 1996. The Essential McLuhan. Edited by Eric McLuhan \& Frank Zingrone. New York: Basic Books.

Mead, George Herbert. 1972. Mind, Self, and Society. From the Standpoint of a Social Behaviorist. Chicago: University of Chicago Press.

Merton, Robert K. 1968. Social Theory and Social Structure. New York: The Free Press.

Miles, Steven. 2006. Consumerism: As a Way of Life.

Miller, Neal C. 1959. "Liberalization of Basic S-R Concepts: Extensions to Conflict Behavior, Motivation and Social Learning." In: Psychology: a Study of a Science. Edited by S. Koch.Vol. 2, 196-292. New York: McGraw-Hill.

Mills, Charles Wright. 2000. The Sociological Imagination. London: Oxford University Press.

Misztal, Bronisław. 2000. Teoria socjologiczna a praktyka społeczna.

Kraków: Universitas.

Modzelewski, Karol. 2004. Barbarzyńska Europa. Warszawa: Iskry. [For English edition see: Modzelewski, Karol. 2015. Barbarian Europe. Translated by Ewa Macura. Frankfurt am Main: Peter Lang.]

Mokrzycki, Edmund. 1980. Filozofia nauki a socjologia. Warsaw: Państwowe Wydawnictwo Naukowe.

Morawska, Elżbieta. 2002. “Socjologia historyczna.” In: Encyklopedia Socjologii, vol. 4, 65-69. Warsaw: Oficyna Naukowa.

Morris, Paul. 1996. “Community Beyond Tradition.” In: P. Heelas, S. Lash, P. Morris (eds.), Detraditionalization. Critical Reflections on Authority and Identity, 223-249. Oxford: Blackwell.

Moscovici, Serge. 1981. “On Social Representations.” In: Social Cognition: Perspectives on Everyday Understanding. Edited by J. P. Forgas. London: Academic Press.

Moscovici, Serge. 1983. “The Coming Era of Representations.” In: J-P. Codol \& J-P. Leyens (eds.), Cognitive Analysis of Social Behaviour. The Hague: Martinus Nijhoff.

Moscovici, Serge \& Robert M. Farr.1983. Social Representations. Cambridge: Cambridge University Press.

Moscovici, Serge. 1985. The Age of the Crowd. A Historical Treatise on Mass Psychology. Cambridge: Cambridge University Press.

Mulliez, Jacques. 1990. “La designation du père." In: Histoire des pères et de la paternité. Edited by J. Delumeau \& D. Roche. Paris: Larousse.

Murray, Charles. 2015. Losing Ground: American Social Policy, 1950-1980. New York: Basic Books. 
Nancy, Jean-Luc. 1991. Inoperative Community. Minneapolis: Minnesota University Press.

Neumann, Erich. 1971. Art and the Creative Unconscious. Translated by Ralph Manheim. Princeton: Princeton University Press.

Nisbet, Robert. 1972. "Introduction: The Problem of Social Change." In: Social Change. Edited by R. Nisbet, 1-45. New York: Harper \& Row.

Nisbet, Robert \& Robert G. Perrin. 1977. The Social Bond. New York: McGraw-Hill.

Nowak, Andrzej. 1996. “Bąble nowego w morzu starego." In: Podstawy $\dot{z}$ ycia społecznego w Polsce. Edited by M. Marody, E. Gucwa-Leśny. Warsaw: ISS UW.

O'Loughlin, Claire \& Paul Thagard. 2000. "Autism and Coherence: A Computational Model." Mind \& Language 15.4: 375-392.

Offe, Claus. 1985. "Divergent Rationalities of Administrative Action." In: Disorganized Capitalism, Contemporary Transformations of Work and Politics. Edited by J. Keane. London: Polity Press.

Offe, Claus. 1998. “Homogeneity” and Constitutional Democracy: Coping with Identity Conflicts through Group Rights." The Journal of Political Philosophy 6.2: 113-141.

Ortega y Gassett, Jose. 1994. The Revolt of the Masses. New York: W. W. Norton \& Company.

Osiatyński, Wiktor. 1986. Preface to: Alvin Toffler, Trzecia fala [The Third Wave], 7-20. Warsaw: PIW.

Ossowska, Maria. 1986. Etos rycerski i jego odmiany. Warsaw: Państwowe Wydawnictwo Naukowe.

Ossowski, Stanisław. 1966. Więź społeczna a dziedzictwo krwi. Dzieła, vol. 2. Warsaw: Państwowe Wydawnictwo Naukowe.

Parsons Talcott \& Alfred Kroeber. 1958. "Concept of Culture and of Social System.” American Sociological Review 23: 852-853.

Parsons, Talcott. 1949. “The Social Structure of Family." In: The Family: its Functions and Destiny. Edited by R. Anshen. New York: Harper \& Brothers.

Parsons, Talcott. 1951. The Social System. London: Routledge \& Kegan Paul. Perrot, Michelle \& Anne Martin-Fugier. 1990. “The Actors.” In: A History of Private Life, vol. 4, From the Fires of Revolution to the Great War, 95-338. Edited by Michel Perrot. Translated by Arthur Goldhammer. Cambridge, Mass.: The Belknap Press of Harvard University Press. 
Piaget Jean. 1979. "Intellectual Evolution from Adolescence to Adulthood." In: Thinking: Readings in Cognitive Science. Edited by P. N. Johnson-Laird \& P. C. Wason, 158-165. Cambridge: Cambridge University Press.

Pinker, Steven. 1998. How the Mind Works. Harmondsworth: Penguin Books. Polanyi, Karl. 2001. The Great Transformation. The Political and Economic Origins of Our Time. Boston: Beacon Press.

Polanyi, Michael. 1967. The Tacit Dimension. London: Routledge \& Kegan Paul.

Poleszczuk, Jan. 1991. "Praca w systemie gospodarki planowej." In: Co nam zostało z tych lat... Społeczeństwo polskie u progu zmiany systemowej. Edited by M. Marody. London: Aneks.

Prigogine, Ilya \& Isabelle Stengers. 1984. Order out of Chaos. Man's New Dialogue with Nature. New York: Bantam Books.

Putnam, Robert D. 1995. "Bowling Alone: America’s Declining Social Capital." Journal of Democracy 6.1: 65-78.

Putnam, Robert D. 2000. Bowling Alone: The Collapse and Revival of American Community. New York: Simon \& Schuster.

Reich, Robert B. 1992. The Work of Nations: Preparing Ourselves for 21st Century Capitalism. New York: Vintage.

Reinsberg, Carola. 1998. Obyczaje seksualne starożytnych Greków. Translated by B. Wierzbicka. Gdynia: Uraeus. [For the German original see: Ehe, Hetärentum und Knabenliebe im antiken Griechenland. Munich: C.H. Beck, 1993.]

Riesman, David. 1969. The Lonely Crowd. New Haven: Yale University Press. Rifkin, Jeremy. 1995. The End of Work. New York: G.P. Putnam's Sons.

Rosenberg, Nathan \& L. E. Birdzell Jr. 1986. How the West Grew Rich: The Economic Transformation of the Industrial World. New York: Basic Books.

Rouche, Michel. 1992. "The Early Middle Ages in the West." In: A History of Private Life, vol. 1, From Pagan Rome to Byzantium, 411-550. Edited by Paul Veyne. Translated by Arthur Goldhammer. Cambridge, Mass.: The Belknap Press of Harvard University Press.

Rousseau, Jean-Jacques. 2002. The Social Contract and The First and Second Discourses. Edited by Susan Dunn. New Haven: Yale University Press.

Saggs, Henry W.F. 1962. The Greatness that was Babylon.

New York: Hawthorne Books.

Sahlins, Marshall D. 1972. “The Original Affluent Society." In: Stone Age Economics, 1-40. Chicago: Aldine.

Schachter,Stanley \& Jerome Singer. 1962. "Cognitive, Social, and Physiological Determinants of Emotional States." Psychological Review 69: 379-399. 
Scribner, Sylvia. 1979. "Modes of Thinking and Ways of Speaking: Culture and Logic Reconsidered." In: P. N. Johnson-Laird \& P. C. Wason (eds.), Thinking: Readings in Cognitive Science, 483-500. Cambridge: Cambridge University Press.

Scruton, Roger. 2002. The West and the Rest: Globalization and the Terrorist Threat. Wilmington: ISI Books.

Searle, John R. 1994. The Rediscovery of the Mind. Cambridge: MIT Press.

Selfe, Lorna. 1985. "Anomalous Drawing Development: Some Clinical Studies." In: Visual Order: the Nature and Development of Pictorial Representation. Edited by N. H. Freeman \& M. V. Cox, 135-154. Cambridge: Cambridge University Press.

Sennett, Richard. 1992. The Fall of Public Man. New York: W.W. Norton \& Company.

Sennett, Richard. 1998. The Corrosion of Character. New York: W. W. Norton \& Company.

Shibutani, Tamotsu. 1986. Social Processes. An Introduction to Sociology. Berkeley: University of California Press.

Shils, Edward. 1997. The Virtue of Civility. Selected Essays on Liberalism, Tradition, and Civil Society. Indianapolis: Liberty Fund.

Shott, Susan. 1979. "Emotion and Social Life. A Symbolic Interactionist Analysis." American Journal of Sociology 84: 1317-1334.

Sikorska, Małgorzata. 2009. Nowa matka, nowy ojciec, nowe dziecko. Warsaw: Wydawnictwa Akademickie i Profesjonalne.

Simmel, Georg. 1950. The Sociology of Georg Simmel. Translated by Kurt H. Wolff. Glencoe: The Free Press.

Simmel, Georg. 2005. The Philosophy of Money. London: Routledge.

Simon, Marcel. 1972. La Civilisation de l'Antiquité et le Christianisme. Paris: Arthaud. [Quoted after the 1992 Polish translation by E.

Bąkowska: Cywilizacja wczesnego chrześcijaństwa. Warsaw: Państwowy Instytut Wydawniczy.]

Skinner, B. F. 2002. Beyond Freedom and Dignity. Indinapolis: Hackett Publishing.

Smith, Adam. 1984. The Theory of Moral Sentiments. Indianapolis: Liberty Fund.

Sober, Elliott \& David Sloan Wilson. 1998. Onto Others. The Evolution and Psychology of Unselfish Behavior. Cambridge: Harvard University Press. Spelke, Elizabeth S. 2009. “Forum.” In: Michael Tomasello, Why We Cooperate, 149-172. Cambridge: MIT Press. 
Stalder, Felix. 2008. Manuel Castells. The Theory of the Network Society. Cambridge: Polity Press.

Stark, David. 2000. For a Sociology of Worth (Keynote address for the Meetings of the European Association of Evolutionary Political Economy, Berlin, November 2-4, 2000). https://pdfs.semanticscholar.org/4594/54a59fef8ed0db 99549c5f7a2a8c26f7cf99.pdf.

Stone, Lawrence. 1977. The Family, Sex and Marriage in England 1500-1800. London: Weidenfeld \& Nicolson.

Stringer, Christopher \& Robin McKie. 1996. African Exodus: The Origins of Modern Humanity. London: Jonathan Cape.

Świderkówna, Anna. 1978. Hellenika. Wizerunek epoki od Aleksandra do Augusta. Warsaw: Państwowy Instytut Wydawniczy.

Swidler, Ann. 1986. "Culture in Action: Symbols and Strategies." American Sociological Review 51: 273-286.

Szacki, Jerzy. 1965. "Durkheim czyli ambicje socjologii." In: Filozofia i socjologia XX wieku, vol. 1, 87-104. Warsaw: Wiedza Powszechna.

Szacki, Jerzy. 2002. Historia myśli socjologicznej. Warsaw: Wydawnictwo Naukowe PWN.

Szawiel, Tadeusz. 1982. "Struktura społeczna i postawy a grupy ethosowe." Studia Socjologiczne 1-2: 157-178.

Szczepański, Jan. 1970. Elementarne pojęcia socjologiczne. Warsaw: Państwowe Wydawnicto Naukowe.

Sztompka, Piotr. 2002. Socjologia. Analiza społeczeństwa. Kraków: Znak.

Tajfel, Henri \& John C. Turner. 1986. “The Social Identity Theory of Intergroup Behaviour." In: S. Worchel \& W. G. Austin (eds.), Psychology of intergroup relations, 7-24. Chicago: Nelson-Hall.

Thomas, William \& Florian Znaniecki. 1918. The Polish Peasant in Europe and America. Boston: Badger.

Thompson, John B. 1995. The Media and Modernity: A Social Theory of the Media. Stanford: Stanford University Press.

Thompson, John B. 2013. Ideology and Modern Culture. Critical Social Theory in the Era of Mass Communication. Stanford: Stanford University Press. E-book.

Tocqueville, Alexis de. 2010. Democracy in America. Edited by Eduardo Nolla. Translated by James T. Schleifer. Indianapolis: Liberty Fund.

Toffler, Alvin. 1989. The Third Wave. New York: Bantam.

Tomasello, Michael. 1999. The Cultural Origins of Human Cognition. Cambridge: Harvard University Press. 
Tönnies, Ferdinand. 2001. Community and Civil Society. Translated by Jose Harris and Margaret Hollis. Cambridge: Cambridge University Press.

Trutkowski, Cezary. 2000. Społeczne reprezentacje polityki. Warsaw: Scholar.

Trutkowski, Cezary. 2002. "Teoria społecznych reprezentacji i jej zastosowania." In: M. Marody. Wymiary życia społecznego. Polska na przełomie XX i XXI wieku, 327-355. Warsaw: Scholar.

Turner, Jonathan H. 2002. The Structure of Sociological Theory. Belmont: Wadsworth.

Vallacher Robin R. \& Andrzej Nowak. 1997. “The Emergence of Dynamical Social Psychology.” Psychological Inquiry 8.2: 73-99.

Veyne, Paul. 1992. “The Roman Empire." In: A History of Private Life: From Pagan Rome to Byzantium. Edited by Paul Veyne. Vol. 1, Translated by Arthur Goldhammer. Cambridge, Mass.: The Belknap Press of Harvard University Press.

Vidal-Naquet, Pierre. 1986. The Black Hunter. Forms of Thought and Forms of Society in the Greek World. Translated by Andrew Szegedy-Maszak. Baltimore: The Johns Hopkins University Press.

Vogt, Joseph. 1967. The Decline of Rome: The Metamorphosis of Ancient Civilisation. Translated by Janet Sondheimer. London: Weidenfeld \& Nicolson.

Vygotski, Lev. 1994. The Vygotsky Reader. Edited by Rene Van der Veer \& Jaan Valsiner. Oxford: Blackwell.

Weber, Max. 1949. "'Objectivity' in Social Science and Social Policy." In: The Methodology of the Social Sciences. Edited by E. Shils \& H. Finch. New York: Free Press.

Weber, Max. 1958. “The Three Types of Legitimate Rule.” Translated by Hans Gerth. Berkeley Publications in Society and Institutions 4.1: 1-11.

Weber, Max. 1978. Economy and Society. An Outline of Interpretive Sociology. Edited by Guenther Roth \& Claus Wittich. Berkeley: University of California Press.

Weber, Max. 2005. The Protestant Ethic and the Spirit of Capitalism. Translated by Talcott Parsons. London: Routledge.

Weeks, Jeffrey. 1989. Sex, Politics and Society. The Regulation of Sexuality since 1800. London: Longman.

Wellman, Beverly \& Barry Wellman. 1992. "Domestic Affairs and Network Relations." Journal of Social and Personal Relationships 9.3: 385-409.

Wicker, Allan W. 1969. "Attitudes versus Actions: The Relationship of Verbal and Overt Behavioral Responses to Attitude Objects." Journal of Social Issues 25: 41-78. 
Wilson, Thomas P. 1970. "Normative and interpretive paradigms in sociology." In: Understanding Everyday Life. Towards the Reconstruction of Sociological Knowledge. Edited by J. D. Douglas. Chicago: Aldine Publishing Company. Wojciszke, Bogdan. 2002. Człowiek wśród ludzi. Zarys psychologii społecznej. Warsaw: Scholar.

Wojda, Monika. 2003. Ekonomiczno-demograficzna analiza zjawiska kohabitacji. MA thesis. Insititute of Sociology at the University of Warsaw.

Wojnarski, Dariusz. 2004. Powszechna historia gospodarcza. Warsaw: Poltext.

Wrong, Denis H. 1961. "The Oversocialized Conception of Man in Modern Sociology." American Sociological Review 26: 183-193.

Wunderlich, Hans Georg. 1974. The Secret of Crete. Translated by Richard Winston. New York: Macmillan.

Wuthnow, Robert. 1987. Meaning and Moral Order. Explorations in Cultural Analysis. Berkeley: University of California Press.

Zientara, Benedykt. 1985. Świt narodów europejskich. Powstawanie świadomości narodowej na obszarze Europy pokarolińskiej. Warsaw: Państwowy Instytut Wydawniczy.

Ziółkowski, Marek. 1981. Znaczenie, interakcja rozumienie. Warsaw: Państwowe Wydawnictwo Naukowe.

Znaniecki, Florian. 2008. Metoda socjologii. Warsaw: Wydawnictwo Naukowe PWN.

Żelazny, Walter. 2004. Etniczność. Ład - konflikt - sprawiedliwość. Poznań: Wydawnictwo Poznańskie. 


\section{INDEX OF NAMES}

A

Abbott, Andrew 27, 339

Achilles 190

Ackerman, Bruce 245

Adorno, Theodor W. 166, 339

Akert, Robin M. 339

Alexander, Jeoffrey C. 332, 339

Amsterdamski, S. 341

Anderson, Benedict 284, 285, 287, $313,324,339$

Anshen, Ruth N. 351

Anthony, Peter D. 339

Appadurai, Arjun 333, 339

Arendt, Hannah 203-206, 214, 215, $220,231,240,245,250-252,254$, 339,340

Ariès, Philippe 70, 260, 280,

281, 339

Arnason, Johann 344

Aronson, Elliot 67, 68, 339

Aristotle 107, 214, 215

Austin, William G. 354

B

Bąkowska, E. 348, 353

Bandura, Albert 74, 339

Barraclough, Geoffrey 105, 339

Baszkiewicz, Jan 275, 339

Bateson, Gregory 192

Batorski, Dominik 16

Bauman, Zygmunt

Beck, Ulrich 33, 36, 37, 132, 196, 197, 200, 201, 203, 226, 230, 234-236, 239, 244, 303, 304, 319, $329,339,340$

Beck-Gernsheim, Elizabeth 18, 32,340
Bell, Daniel 30, 296, 240

Benderly, Beryl L. 41, 68, 345

Benedict, Ruth 88, 192, 284, 340

Benedict, Saint 247

Benhabib, Seyla 245, 250, 251, 253, 289, 340

Berger, Peter L. 47, 60, 97, 110, $112,130,145,146,148,190,264$, 265,340

Berne, Eric 52

Bernstein, Basil 101, 340

Bielicki, Marian 213, 214, 340

Bieńkowska, W. 343

Birdzell, L. E. 352

Birrell, Jean 343

Blackmore, Susan 137, 341

Bloom, Allan 319

Blumer, Herbert 48, 61, 154, 341

Boas, Franz 176, 178

Bogucka, Maria 185, 341

Borkowska, Stanisława 344

Bourdieu, Pierre 107, 114, 120, 144, 181, 258, 303, 304, 325, 341

Braudel, Ferdinand 22, 227, 230, 317, 341

Brenner, Robert 228, 229, 341

Bronowski, Jacob 97-99, 104, 227, 320, 321, 341

Bruner, J.S. 66, 345

Budnikowski, Tomasz 244, 341

Burge, Oscar 344

Burgess, Ernest W. 165, 341

C

Cabantous, Andre 188, 341

Camic, Charles 43, 341

Campbell, G.L. 346 
Caporael, Linnda 108, 132-139, $144,145,149,155,158,159,162$, 262,342

Castaneda, Carlos 57, 342

Castells, Manuel 32, 88, 90, 107, 148, $288,297,301,329,333,342,354$

Charbonnier, Georges 160, 263, $268,307,342$

Chartier, Roger 339

Chaunu, Pierre 182, 187, 342

Chirot, Daniel 319, 341

Chojnowska, Alina 238, 342

Chomsky, Noam 62

Chouraqui, André 213, 342

Chwe Suk-Young, Michael 255, 342

Codol, Jean-Paul 350

Cole, Margaret J. 142, 342

Collins, Randall 260, 265, 342

Comte, August 22, 78-80, 254, 342

Coulanges, Futel do 279

Cox, Maureen V. 353

Crozier, Michael 131, 342

Cicero 215, 247

Cyrus 212

Czarnowski, Stefan 109, 110, 342

D

Dahrendorf, Ralph 78, 319, 335, 342

Daly, Martin 179, 342

Damasio, Antonio R. 66-68, 70, 71, $84,99,115,342$

Darwin, Karol 134, 138, 254

Daszkowski, Julian 234

Deacon, Terrence W. 54, 273, 342

Delumeau, Jean 186, 341, 350

Dennett, Daniel C. 63-66, 70, 73, $75,84,91,95,96,98,100,103$, $108,114,342$

Diamond, Jared 42, 49, 50, 102, 104, 105, 155-157, 160, 179, 207-209, 211,343
Dilthey, Wilhelm 292

Douglas, Jack D. 356

Drucker, Peter F. 301, 343

Duby, Georges 181, 247, 248, 277, 278, 280, 323

Dunbar, Robin 50, 52, 55, 343

Durkheim, Émile 11, 13, 15, 17, 19, $20,22-25,32,33,36-39,41,43,50$, $53,54,58-60,72,76,79,80,83,143$, $149,203,210,224,226,264,265$, 296, 317, 319, 331, 332, 343, 354

Duvall, Evelyn Millis 192

E

Edelman, Gerald M. 73, 343

Edison, Thomas 105

Ecclesiastes 186

Eliade, Mircea 57, 343

Elias, Norbert 17, 34, 80, 99, 100, $117,123,124,127-129,219,250$, 260, 280, 294, 296, 299, 300, 303, $304,307,323,343$

Emirbayer, Mustafa 128, 344

Engels, Friedrich 178, 189, 349

Engeström, Yrjö 142, 342

Erikson, Erik 192

Eversley, David Edward Ch. 346

F

Farr, Robert M. 143, 350

Finch, Janet 192, 344, 355

Fisher, Helen E. 184, 344

Fitoussi, Jean-Paul 17, 344

Flandrin, Jean-Louis $167,170,175$, $180,184,186,344$

Flaubert, Gustave 190

Ford, Henry 234, 237

Forgas, Joseph P. 350

Foucault, Michel 21, 174, 232, 233, 261, 344

Frank, Lawrence K. 191 
Frankfort-Nachmias Chavy 294, 344

Freeman, Norman H. 353

Frenkel-Brunswik, Else 339

Freud, Sigmund 45, 68, 200, 286

Friedberg, Erhard 131, 342

Frieske, Kazimierz W. 242, 344

G

Galerius 274

Gauchet, Marcel 75, 76, 122, 169, 309-312, 314, 315, 324, 344

Gawin, Magdalena 167, 344

Gehlen, Arnold 53, 54, 92, 344

Gerth, Hans 355

Giddens, Anthony 29, 32, 33, 61, $82,86,87,111,130,132,148,196$, 197, 295, 301, 316, 319, 344

Giglioli, Pier P. 345

Gillis, John R. 21, 344

Gilmore, David D. 178, 344

Giza-Poleszczuk, Anna 16, 93, 100, $117,145,166,171,198,200,304$, 315,345

Glass, David V. 346

Gödel, Kurt 146

Goffman, Erving 154, 260, 345

Goldhammer, Arthur 339

Goody, Jack 99, 100, 115,184, 345

Gould, Stephen J. 62, 345

Graves, Robert 269, 345

Greenfield, Patricia M. 66, 345

Greenspan, Stanley I. 41, 68-70, 74-76, 198, 286, 345

Gregory, Richard 103, 185, 192, 276

Gromkowska, Agnieszka 147, 345

Gregory VII 185

Gregory of Tours 276

Gucwa-Leśny, Ewa 349, 351

Guille-Escuret, Georges 41, 44, 50-55, $58,59,62,64,73,101,106,346$

Guizot, François P.G. 169, 190
$\mathrm{H}$

Habermas, Jurgen $86,87,245$, 251-254, 282, 298, 340, 346

Hajnal, John 187, 346

Hall, Catherine 190, 347

Halls, W.D. 343

Hammurabi 269

Harré, Rom 41, 143

Hartley, Jean 341, 346

Harvey, David 107, 241, 242, 341, 346

Hausner, Jerzy 331, 346

Heelas, Paul 340, 350

Hegel, Georg Wilhem F. 190, 253

Heisenberg, Werner 106, 346

Hesiod 215, 269

Hobbes, Thomas 22, 81, 346

Hobhouse, Henry 209, 346

Homer 177, 214, 215

Hopman, F. 347

Huizinga, Johan 222, 229, 280, 346

Hull, David L. 135, 137-139, 155, 162,347

Hume, David 204

Humphrey, Nicholas 54-56, 59, 347

Hurley, Robert 344

Hunt, Lynn 190, 285, 347

Hutchins, Edwin 142, 347

I

Inglehart, Ronald 31, 347

J

Jackobson, Dan 346

Jacobson-Widding, Anita 148, 347

James, William 13, 57, 273, 347

Jasińska-Kania, Aleksandra 345

Jephcott, Edmund 343

Joas, Hans 25, 347

Johnson-Laird, Philip N. 352, 353 
K

Kant, Immanuel 81

Kapuściński, Ryszard 77

Kaufmann, Jean-Claude 48, 53, 59, $61,62,108,110,111,127,134,347$

Keane, Judith 351

Kempny, Marian 16

Klandermans, Bert 346

Knobl, Wolfgang 25, 347

Knorr-Cetina, Karin 315, 347

Koch, Sigmund 350

Kochanowicz, Jacek 16, 161, 267, 299, 347

Kohlberg, Lawrence 87, 347

Kohn, Melcin L. 144, 166, 347

Konecki, Krzysztof 144, 347

Koralewicz-Zębik, Jadwiga 347

Kroeber, Alfred L. 43, 351

Kuhn, Thomas S. 306, 309, 348

Kula, Witold 21, 181, 198, 348

Kumar, Krishan 32, 348

Kymlicka, Will 85, 251, 314, 348

L

Lasch, Christopher 82, 167, 192-195, 331,348

Lash, Scott 238, 340, 348, 350

Laslett, Peter 167, 175, 348

Lawrence, Frederick 346

Le Bon, Gustave 265, 348

Le Goff, Jacques 217-219, 220-223, 225, 227, 307, 348

Ledoux, Joseph 13

Lee, Nick 340

Lelong, Charles 277, 348

Lem, Stanisław 105, 141

Lenin, Vladimir 167, 297

Lévi-Strauss, Claude 160, 178, 263, 268, 307, 342, 348

Levine, David 167, 170, 172, 175 , 189, 190, 348, 349

Levinson, Daniel J. 339
Lewis-Williams, David 56, 57, 59, 73, 102, 349

Leyens, Jacques P. 350

Littré Maximilien, P.E. 248

Livingstone, Sonia M. 237

Locke, Harvey J. 12

Locke, John 341

Luckmann, Thomas 47, 60, 97, 110, $112,130,145,146,264,340$

Louis XIV 282

Lunt, Peter K. 237

Luther, Martin 147

Łoś, Maria 121, 349

M

Maciejewski, Marek 230, 231, 349

Macura, Ewa 350

Maffesoli, Michel 329, 349

Malinowski, Broniław 100, 349

Malthus, Thomas R. 169, 189, 301

Mandes, Sławomir 16

Marx, Karl 22, 29, 32, 167, 200, 204, 208, 226, 296, 317, 349

Marody, Mirosława 93, 94, 96, 99, $100,107,114,129,153,345-347$, 349, 351, 352, 355

Martin-Fugier, Anne 165-169, 173, 186-190, 199, 233, 234, 242, 349, 350, 351

Mauss, Marcel 91, 349

McCarthy, Thomas 346

McKie, Robin 49, 50, 92, 354

McLuhan, Marshall 107, 288, 303, 333, 349, 350

McQuail, Denis 339

Mead, George Herbert

Mead, Margaret 13, 46-48, 53, 59-61, 73, 95, 96, 127, 128, 154, $178,191,341,350$

Merton, Robert K. 121, 130, 350

Mikołejko, Zbigniew 16, 273

Miles, Steven 237, 350 
Miller, Neil E. 294, 350

Mills, C. Wright 29, 43, 291, 350

Milligan, Martin 349

Mische, Ann 128, 344

Misztal, Bronisław 30, 350

Modzelewski, Karol 217, 218, 268, 275-277, 299, 350

Mokrzycki, Edmund 291, 294, 350

Mokyr, Joel 297

Morawska, Elżbieta 294, 350

Morris, Paul 311, 325, 326, 336, 340,350

Moscovici, Serge 143, 285, 286, 308, 350

Mulliez, Jacques 171, 350

Munro, Rolland 340

Murray, Charles 195, 350

$\mathbf{N}$

Nachmias, David 294, 344

Nadia 55, 56

Nancy, Jean-Luc 82, 83, 85, 229, 337, 351

Neumann, Erich 54, 351

Newton, Issac 98, 292

Nisbet, Robert 77, 334, 351

Nowak, Andrzej 147, 351, 355

O

Offe, Claus 237, 343, 314, 351

Ogden, Charles K. 349

O'Loughlin, Claire 56, 351

Ortega y Gasset, José 328, 351

Osiatyński, Wiktor 319, 351

Ossowska, Maria 280, 351

Ossowski, Stanisław 20, 78, 123, $174,183,351$

$\mathbf{P}$

Parsons, Talcott 25, 26, 28-30, 33, $43,44,93,128,131,165,341$, 351,355
Passeron, Jean-Claude 114, 121, 262, 290, 325, 341

Paul, St. 184, 185, 220, 273

Perrin, Robert G. 77, 351

Perrot, Michelle 165, 167-169, 173, 186-190, 199, 233, 234, 347, 351

Piaget, Jean 66, 352

Picasso, Pablo 49, 92

Pinker, Steven 62, 66, 199, 352

Polanyi, Karl 228, 352

Polanyi, Michael 115, 352

Poleszczuk, Jan 16, 104, 203, 352

Prigogine, Ilya 292, 293, 352

Putnam, Robert D. 319, 332, 352

$\mathbf{R}$

Reich, Robert 240, 301, 352

Reinsberg, Carol 177, 352

Reynold, Siân 341

Richards, Ivor A. 349

Richardson, Jeffrey G. 341

Richelieu, Armand Jean du Plessis cardinal de 281

Rickert, Heinrich 292

Riesman, David 124, 307, 352

Rifkin, Jeromy 243, 352

Ritter, Mark 340

Roberts, David 344

Robespierre, Maximilien 285

Roche, Daniel 186, 341, 350

Rogaczewska, Maria 16, 270

Rosanvallon, Pierre 17, 344

Rosenberg, Nathan 233, 352

Rouche, Michel 280, 352

Rousseau, Jean-Jacques 22, 82, 352

$S$

Saggs, Henry W.F. 209, 212-214, 269, 352

Sahlins, Marshall D. 94, 206-208, 352

Saint-Simon, Claude Henri de 22 
Salomon, Gavriel 342

Saussure, Ferdinand de 23

Schachter, Stanley 68, 352

Schleifer, James T. 354

Schröter, Michael 343

Schooler, Carmi 144, 166, 347

Scribner, Silvia 66, 353

Scruton, Roger 311, 353

Searle, John R. 63, 353

Selfe, Lorna 55, 353

Sennett, Richard 235, 239, 242, 255, 260, 261, 319, 353

Sheridan, Alan 343

Shibutani, Tomotsu 128, 353

Shils, Edward 332, 353, 355

Shott, Susan 323, 353

Simmel, George 11, 19, 20, 35-39, $43,74,79,121,141,143,203,353$

Simon, Marcel 274, 353

Singer, Jerome 68,352

Skinner, Burrhus F. 108, 136, 347, 353

Smith, Adam 79, 80, 132, 133, 229, 254,353

Sober, Elliot 138, 353

Sombart, Werner 226

Sondheimer, Janet 355

Spencer, Herbert 22

Spinoza 84

Spock, Benjamin 194

Stalder, Felix 32, 354

Stark, David 43, 225, 354

Stengers, Isabelle 292, 293, 352

Stringer, Christopher 49, 50, 92, 354

Suk-Young Chwe, Michael 255, 342

Świderkówna, Anna 270, 354

Swidler, Ann 92-95, 354

Szacki, Jerzy 22, 23, 33, 35, 38, 43, $91,165,292,354$

Szawiel, Tadeusz 273, 354

Szczepański, Jan 77, 354

Szegedy-Maszak, Andrew 355

Sztompka, Piotr 25, 71, 75, 130, 354
$\mathrm{T}$

Tajfel, Henri 148, 354

Thagard, Paul 56, 351

Thomas, William I. 354

Thompson, John B. 284, 285, 324,354

Tocqueville, Alexis de 167, 190, 354

Toffler, Alvin 231, 232, 236, 351, 354

Tomasello, Michael 46, 51, 66, 69, $70,74,98,256,353,354$

Thomas Aquinas 220, 221

Tönnies, Ferdinand 11, 19, 22, 36, $39,76,79,83-88,90,91,93,100$, $116,118,120-122,165,201,210$, 289, 302, 355

Trask, Williard R. 343

Trutkowski, Cezary 143, 355

Turner, J.C. 148, 354

Turner, Jonathan H. 61, 67, 355

U

Ure, Andrew 230

Urry, John 238, 348

V

Vallacher, Robin R. 147, 355

van Vuuren T. 346

Veblen, Thorstein 237

Veyne, Paul 215-217, 352, 355

Vico, Giambattista 344

Vidal-Naquet, Pierre 77, 214, 215, 269, 270, 355

Vogt, Joseph 271, 272, 355

Vygotski, Lev 53, 355

W

Wakar, K. 347

Wallerstein, Immanuel 6

Walters, Richard H. 74, 339

Wason, Peter C. 352, 353

Watt, Ian P. 100, 105, 345 
Weber, Max 11, 12, 22-24, 32, $73,74,86,92,93,127,128,145$, $147,203,210,220,225,226,236$, 267, 272, 281, 292, 298, 300, 307, 327,355

Weeks, Jeffrey 167, 355

Wellman, Barry 90, 355

Wellman, Beverly 90, 355

Wicker, Allan W. 27, 355

Wilkin, Jerzy 153, 349

Wilson, David Sloan 138, 353

Wilson, K. Everett 343

Wilson, Margo 179, 342

Wilson, Thomas P. 26, 153

Wilson, Timothy D. 339

Wojciszke, Bogdan 27, 356
Wojda, Monika 198, 356

Wojnarski, Dariusz 227, 356

Wolff, Kurt H. 353

Wolicki, K. 346

Worchel, Stephen 354

Wrong, Denis H. 44, 95, 356

Wunderlich, Hans Georg 267, 270, 356

Wuthnow, Robert 92, 93, 356

$\mathbf{Z}$

Żelazny, Walter 270, 284, 313, 356

Zientara, Benedykt 284

Ziółkowski, Marek 47, 48, 356

Zirk-Sadowski, Marek 16, 271

Znaniecki, Florian 18, 28, 354, 356 